NSR\&D Program Fiscal Year 2014

Funded Research

\title{
Stochastic Modeling of Radioactive Material Releases
}

Idaho National Laboratory - Nuclear

Energy \& Idaho State University

September 2015

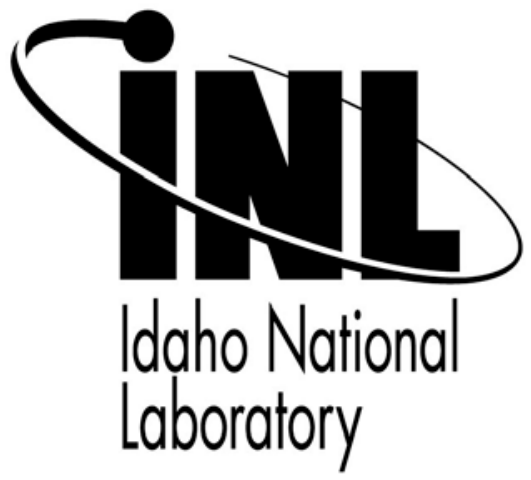

INL is a U.S. Department of Energy National Laboratory operated by Battelle Energy Alliance 


DISCLAIMER
This information was prepared as an account of work sponsored by
an agency of the U.S. Government. Neither the U.S. Government nor any
agency thereof, nor any of their employees, makes any warranty, expressed
or implied, or assumes any legal liability or responsibility for the accuracy,
completeness, or usefulness, of any information, apparatus, product, or
process disclosed, or represents that its use would not infringe privately
owned rights. References herein to any specific commercial product,
process, or service by trade name, trade mark, manufacturer, or otherwise,
does not necessarily constitute or imply its endorsement, recommendation,
or favoring by the U.S. Government or any agency thereof. The views and
opinions of authors expressed herein do not necessarily state or reflect
those of the U.S. Government or any agency thereof.


NSR\&D Program Fiscal Year 2014 Funded Research

Stochastic Modeling of Radioactive Material Releases

Revision 0
September 2015

Idaho National Laboratory - Nuclear Energy \& Idaho State

University

\author{
Prepared for the \\ U.S. Department of Energy \\ Assistant Secretary for Nuclear Energy \\ Under DOE Idaho Operations Office \\ Contract DE-AC07-05ID14517
}




\section{EXECUTIVE SUMMARY}

Nonreactor nuclear facilities operated under the approval authority of the U.S. Department of Energy (DOE) use unmitigated hazard evaluations to determine if potential radiological doses associated with design basis events challenge or exceed dose evaluation guidelines. Unmitigated design basis events that sufficiently challenge dose evaluation guidelines or exceed the guidelines for members of the public or workers merit selection of safety structures, systems, or components (SSCs) or other controls to prevent or mitigate the hazard.

Idaho State University, in collaboration with Idaho National Laboratory, has developed a portable and simple to use software application called SODA (Stochastic Objective Decision-Aide) that stochastically calculates the radiation dose associated with hypothetical radiological material release scenarios. Rather than producing a point estimate of the dose, SODA produces a dose distribution result to allow a deeper understanding of the dose potential. SODA allows users to select the distribution type and parameter values for all of the input variables used to perform the dose calculation. SODA then randomly samples each distribution input variable and calculates the overall resulting dose distribution. In cases were an input variable distribution is unknown, a traditional single point value can be used. SODA was developed using the MATLAB coding framework. The software application has a graphical user input. SODA can be installed on both Windows and Mac computers and does not require MATLAB to function.

SODA provides improved risk understanding leading to better informed decision making associated with establishing nuclear facility MAR limits and safety SSC selection. It is important to note that SODA does not replace or compete with codes such as MACCS or RSAC, rather it is viewed as an easy to use supplemental tool to help improve risk understanding and support better informed decisions. The work was funded through a grant from the DOE Nuclear Safety Research and Development Program. 


\section{Contents}

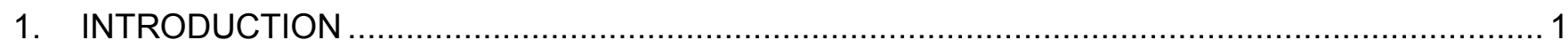

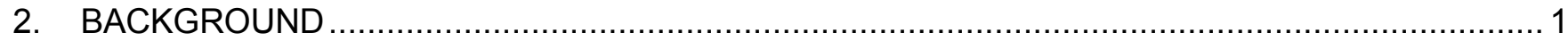

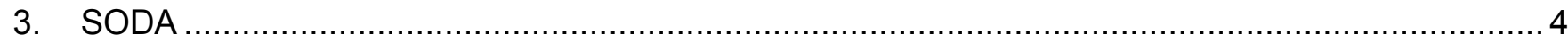

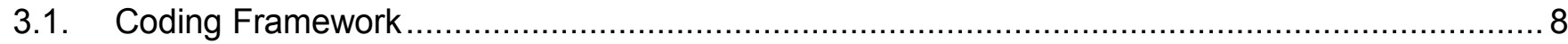

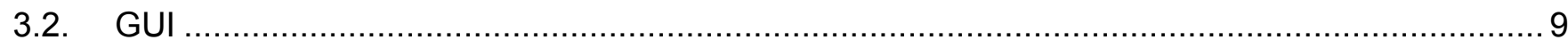

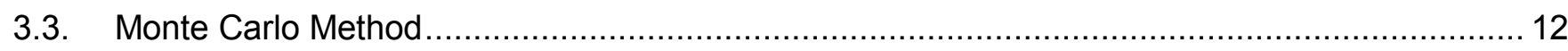

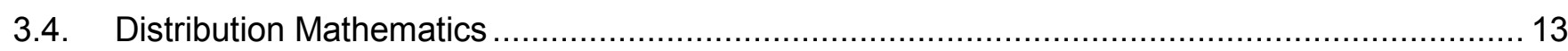

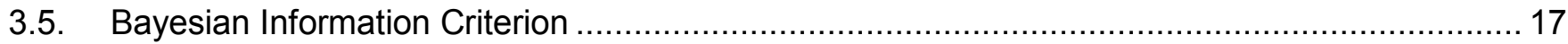

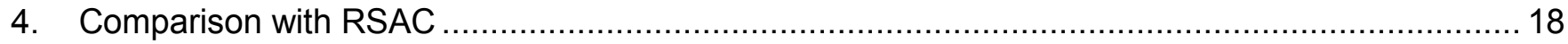

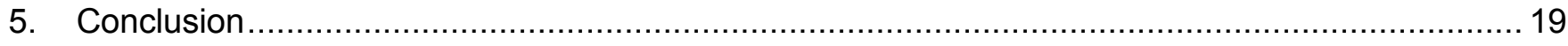

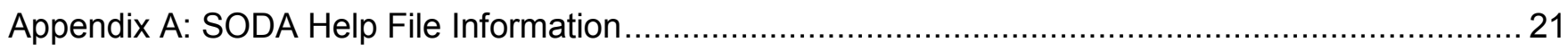

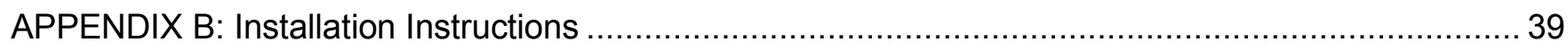

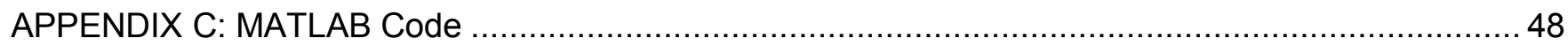

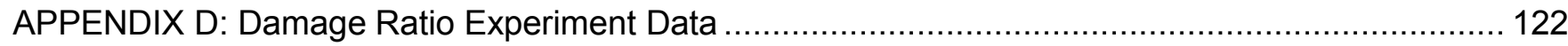

\section{Figures}

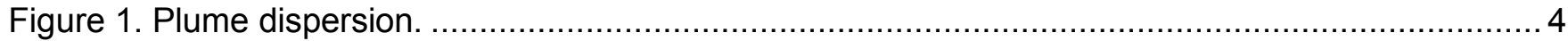

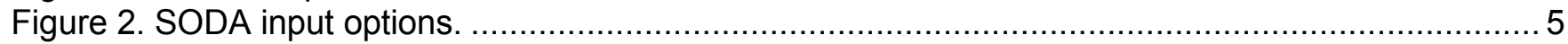

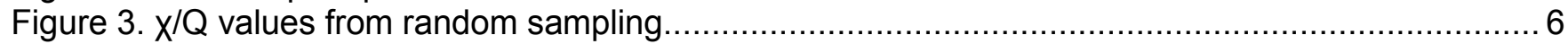

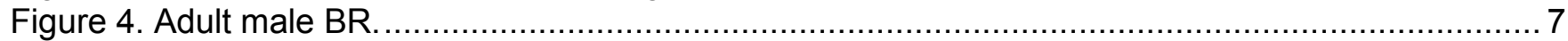

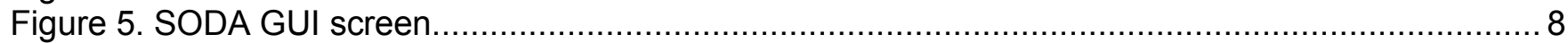

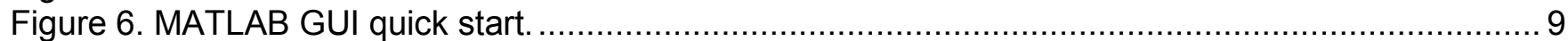

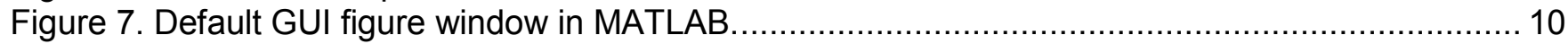

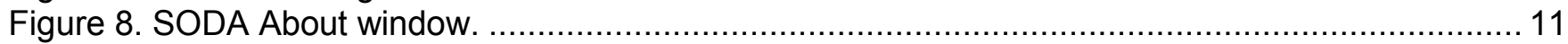

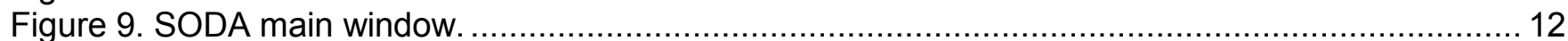

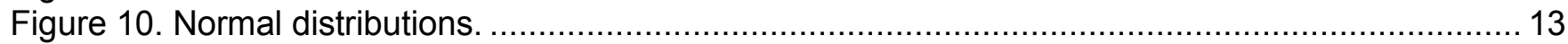

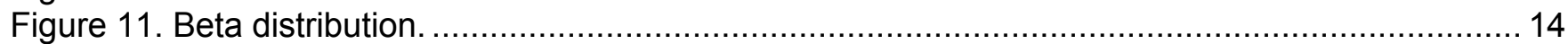

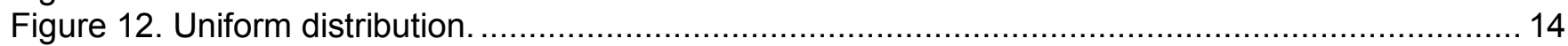

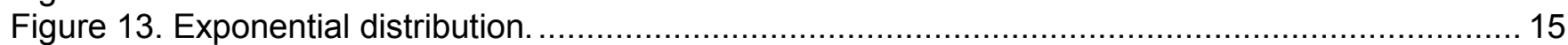

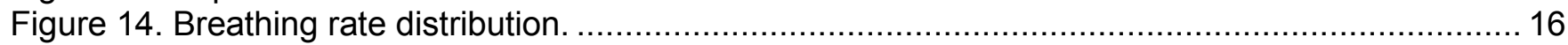

Figure 15. Example grapnhical representation of BIC fit to a randomly generated dose distribution..... 18

Tables

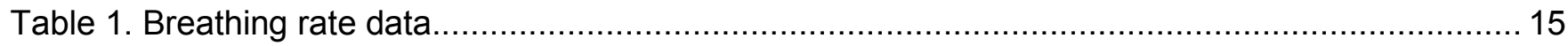

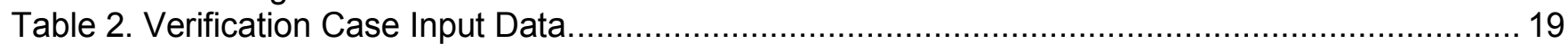

Table 3. Verification Results Comparison. ....................................................................... 19 


\section{ACROYNMS}

$\begin{array}{ll}\text { ARF } & \text { airborne release fraction } \\ \text { BIC } & \text { Bayesian Information Criterion } \\ \text { BR } & \text { breathing rate } \\ \text { CED } & \text { committed effective dose } \\ \text { DBE } & \text { design basis event } \\ \text { DCF } & \text { dose conversion factor } \\ \text { DOE } & \text { Department of Energy } \\ \text { DR } & \text { damage ratio } \\ \text { GUI } & \text { graphical user interface } \\ \text { GUIDE } & \text { Graphical User Interface Development Environment } \\ \text { ICRP } & \text { International Commission on Radiological Protection } \\ \text { IDE } & \text { integrated development environment } \\ \text { INL } & \text { Idaho National Laboratory } \\ \text { ISU } & \text { Idaho State University } \\ \text { LPF } & \text { leak path factor } \\ \text { MACCS } & \text { MELCOR Accident Consequence Code System } \\ \text { MAR } & \text { material-at-risk } \\ \text { NSRD } & \text { Nuclear Safety Research and Development } \\ \text { PDC } & \text { probability density curve } \\ \text { RF } & \text { respirable fraction } \\ \text { RSAC } & \text { Radiological Safety Analysis Computer } \\ \text { SODA } & \text { Stochastic Objective Decision Aide } \\ \text { SSC } & \text { system, structure, or component } \\ \text { ST } & \text { source term } \\ & \end{array}$




\section{INTRODUCTION}

Nonreactor nuclear facilities operated under the approval authority of the U.S. Department of Energy (DOE) use unmitigated hazard evaluations to determine if potential radiological doses associated with design basis events (DBEs) challenge dose evaluation guidelines. Unmitigated DBEs that sufficiently challenge dose evaluation guidelines for members of the public or workers merit selection of safety structures, systems, or components (SSCs) or other controls to prevent or mitigate the hazard.

Idaho State University (ISU), in collaboration with Idaho National Laboratory (INL), has developed a portable and simple to use software application called SODA (Stochastic Objective Decision-Aide) that utilizes a Monte Carlo based code system to stochastically calculate the radiation dose of hypothetical radiological material release scenarios. Rather than producing a point estimate of the dose, SODA produces a dose distribution to allow a deeper understanding of the dose potential. Thus, SODA provides improved risk understanding leading to better informed decision making associated with establishing material-at-risk (MAR) limits and safety SSC selection. It is important to note that SODA does not replace or compete with codes such as MELCOR Accident Analysis Code System (MACCS) or Radiological Safety Analysis Computer (RSAC); rather it is viewed as an easy-to-use supplemental tool to help improve risk understanding and support better informed decisions. The work was funded through a grant from the DOE Nuclear Safety Research and Development (NSRD) Program.

\section{BACKGROUND}

Traditional radioactive material release modeling codes typically provide a bounding single point estimate of receptor dose. While this approach attempts to bound the dose estimate, at least in the context of the atmospheric dispersion model, it falls short in providing quantification of the expected value and the uncertainty associated with the dose estimate. This is particularly problematic when one considers the lack of governing distribution identification for input parameters. Thus, potential doses to members of the public are frequently overstated, leading to excessively conservative MAR limits and potentially over selection of safety SSCs.

DBE dose consequence calculations traditionally use the "five-factor" formula:

$$
S T=M A R \cdot D R \cdot A R F \cdot R F \cdot L P F
$$


where $S T$ is the source term $(\mathrm{Bq})$, MAR is the total available material-at-risk $(\mathrm{Bq})$, DR is the damage ratio (no units), ARF is the airborne release fraction (no units), RF is the respirable fraction (no units), and LPF is the leak path factor (no units).

Potential radiation doses are then calculated using:

$$
C E D=\chi / Q \cdot B R \cdot S T \cdot D C F
$$

where CED is the committed effective dose (Sv), $X / Q$ is the plume dispersion $\left(s / \mathrm{m}^{3}\right), B R$ is the breathing rate $\left(\mathrm{m}^{3} / \mathrm{s}\right)$, ST is the source term $(\mathrm{Bq})$, and $\mathrm{DCF}$ is the dose conversion factor $(\mathrm{Sv} / \mathrm{Bq})$.

The ST is defined as the amount of radioactive or hazardous material released to the environment following an accident. Quantifying the radiation source term goes beyond the determination of the different radionuclides involved. Understanding how the radionuclide reacts with the environment is vital if the source term is to be accurate. The effect of barriers and waste containers play a significant role in decreasing the uncertainties. With considering the uncertainties involved, obtaining an accurate value for the ST is challenging. Most ST values are usually bounding or worst case scenarios; this means that MAR, DR, ARF, RF, and LPF are selected based on bounding estimates to produce a very bounding ST. This method of estimation, while conservative, does not provide a particularly meaningful result.

MAR is the amount of radioactive material available to be acted upon as a result of a physical disturbance such as a spill, shock, or fire. The MAR can also be defined as the value representing a maximum quantity of radioactive material present or anticipated from the analysis of a structure. Thus, the MAR associated with a facility explosion would be different from the MAR during the spill of a radioactive material powder. For instance if an earthquake were to occur at a nuclear facility, the MAR would be everything in the nuclear facility because the earthquake impacts the entire facility.

DR is the fraction of the MAR that is effected by the accident scenario. For example, if a facility holds many containers of radioactive material and a seismic event occurs, a DR could be applied indicating that only a portion of the containers are subjected to enough seismic impact to result in the release of radioactive material.

ARF is employed in the estimation of the fraction of radioactive materials suspended in air as an aerosol, thus available for transport due to physical stress from a specific accident.

$\mathrm{RF}$ refers to the quantity of released material that has an aerosol particle size such that it can penetrate into the alveolar region of the lungs and be deposited. Large particles tend to deposit before they get 
deep into the lung, where they can be expelled through normal body processes. Very small particles will get into the alveolar region of the lung, but they tend to remain in air suspension rather than being deposited into the lung. These particles are typically expelled in later breaths. Particles of intermediate size are able to get into the alveolar region, but are heavy enough to deposit deep in the lung. The fraction of the material that is suspended in air, having this particle size, is given by the RF.

Chi over $\mathrm{Q}(\chi / Q)$ is a normalized air concentration term, expressed in seconds per cubic meter. This is used to quantify the effect of diffusion on a plume as it propagates downwind. As calculated, it is an expression of radioactivity per cubic meter, per radioactivity per second. $Q$ is the material release rate term (radioactivity per second). Since Chi depends on $Q$, dividing out $Q$ normalizes to the release rate, allowing the term to describe the rate of plume dispersion, independent of release rate. Typically, a higher $\chi / Q$ will result in a higher potential dose to a population.

BR allows the model to account for the difference in respiration rate of a person who is exposed depending on their activity state. Over a day, a given person spends some time sleeping, some time awake and inactive, and some time active. Using this 24-hour data for typical respiration rate, and sampling this data, the distribution of the resulting dose is influenced. This allows decision makers to ascertain the full range of potential doses to a population.

DCF is the multiplicative factor relating activity to absorbed dose. A person exposed to material of some activity is going to receive a different absorbed dose dependent upon the type of radiation, where in or on the body that the exposure is occurring, and other factors. The traditional point values used are obtained from the International Commission on Radiological Protection (ICRP).

Conservative single value input parameters are typically used to represent ARF, RF, LPF, and BR. The traditional methodology, while conservative, can lead to skewed conclusions in the balance between cost and risk reduction resulting in over engineered systems with greater design, construction, and operating costs. Rather than using a bounding single point value for each parameter in the dose consequence calculation, distributions for some or all of the parameters can be used. For example, Figure 1 shows how radioactive material can disperse as it transports away from the event site. Independent parameters affecting the concentration profile can be stochastically sampled and used in the $X / Q$ portion of the dose calculation. 


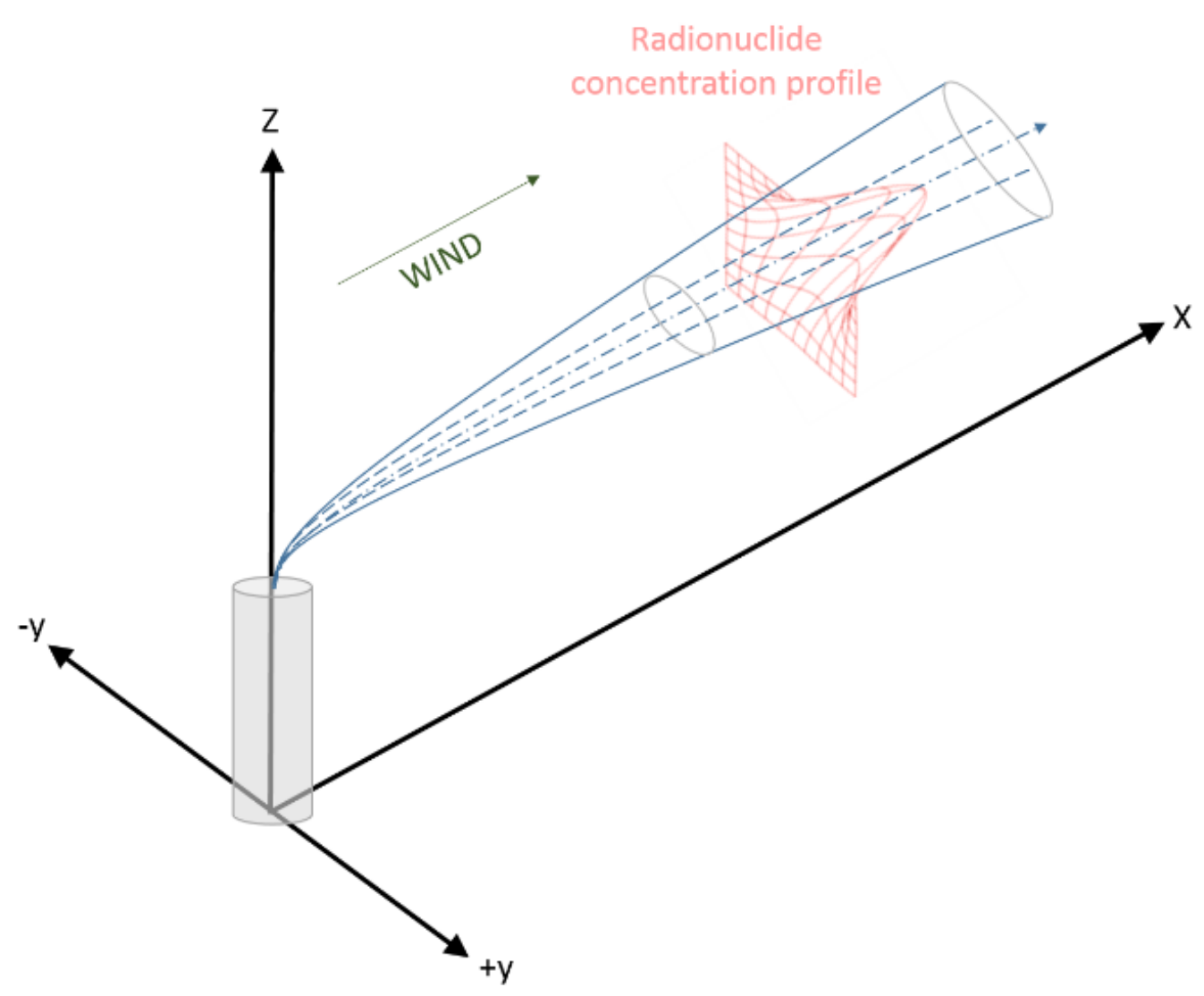

Figure 1. Plume dispersion.

Each parameter distribution can be stochastically sampled, and the resulting dose consequence calculation can be repeated many times to develop a dose consequence distribution. The resulting dose distribution can then be used by decision makers to make a better informed decision about how a particular DBE challenges dose evaluation guidelines.

\section{SODA}

The software application is not intended to replace or even compete with traditional radioactive material release modeling codes such as the MACCS or RSAC code; rather it is viewed as a simple to use supplement to help improve risk understanding. The application was developed using MATLAB computing environment and programming language, and it incorporates use of Monte Carlo techniques as well as a graphical user interface (GUI). The code system also utilizes MATLAB vectorization to provide execution time reduction. The application includes user selection of the governing distribution for parameters as the MAR, DR, ARF, RF, LPF, BR, and DCF. While MATLAB was used for the development work, the application is distributed as a self-contained executable program. As seen in 
Figure 2, features of the application include pull down menus with available distributions for the various parameters.

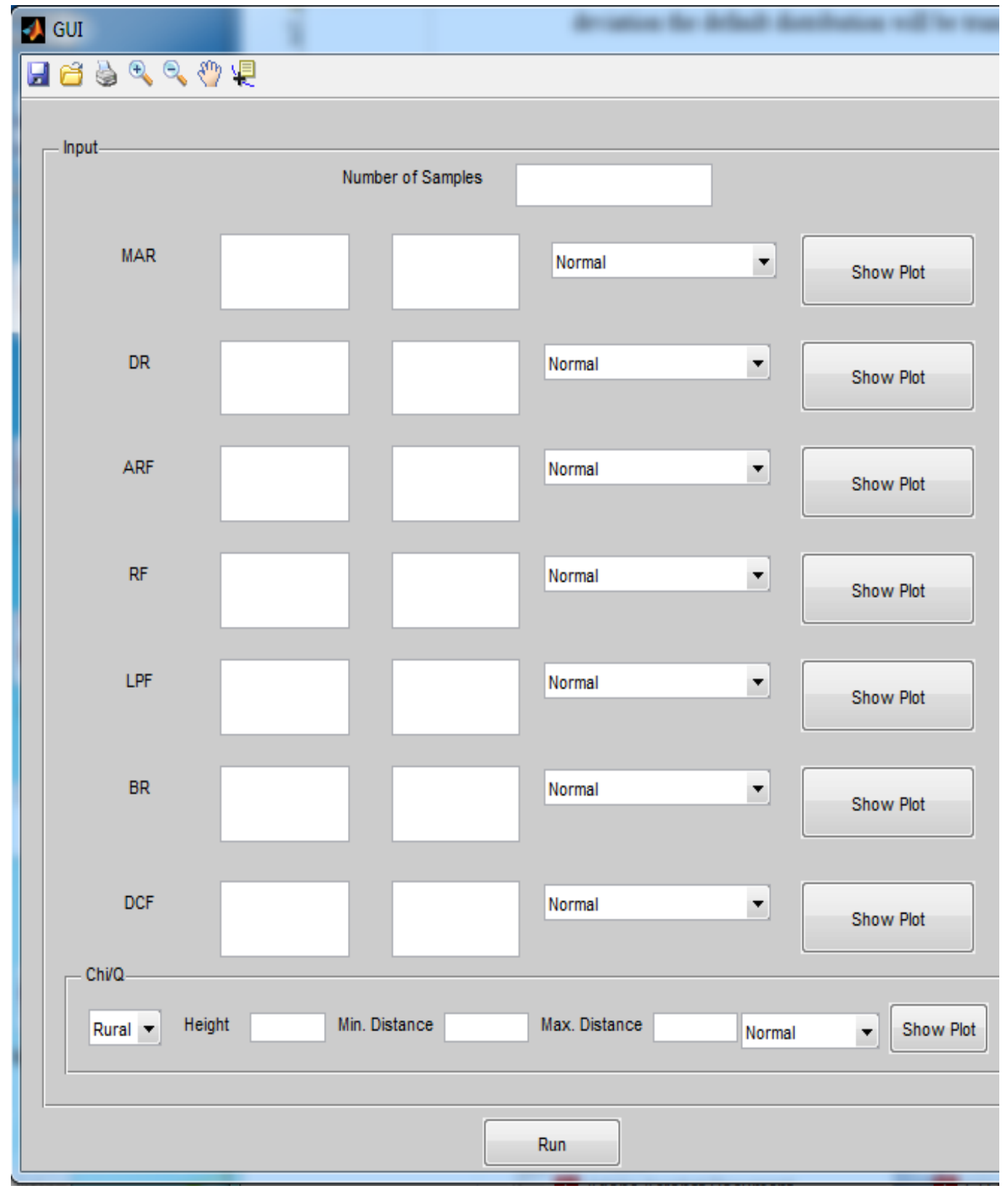

Figure 2. SODA input options.

The user has the option to plot the input parameter distribution that results from the random sampling process. For example, Figure 3 shows a sample distribution of $X / Q$ values resulting from one million random samples. 


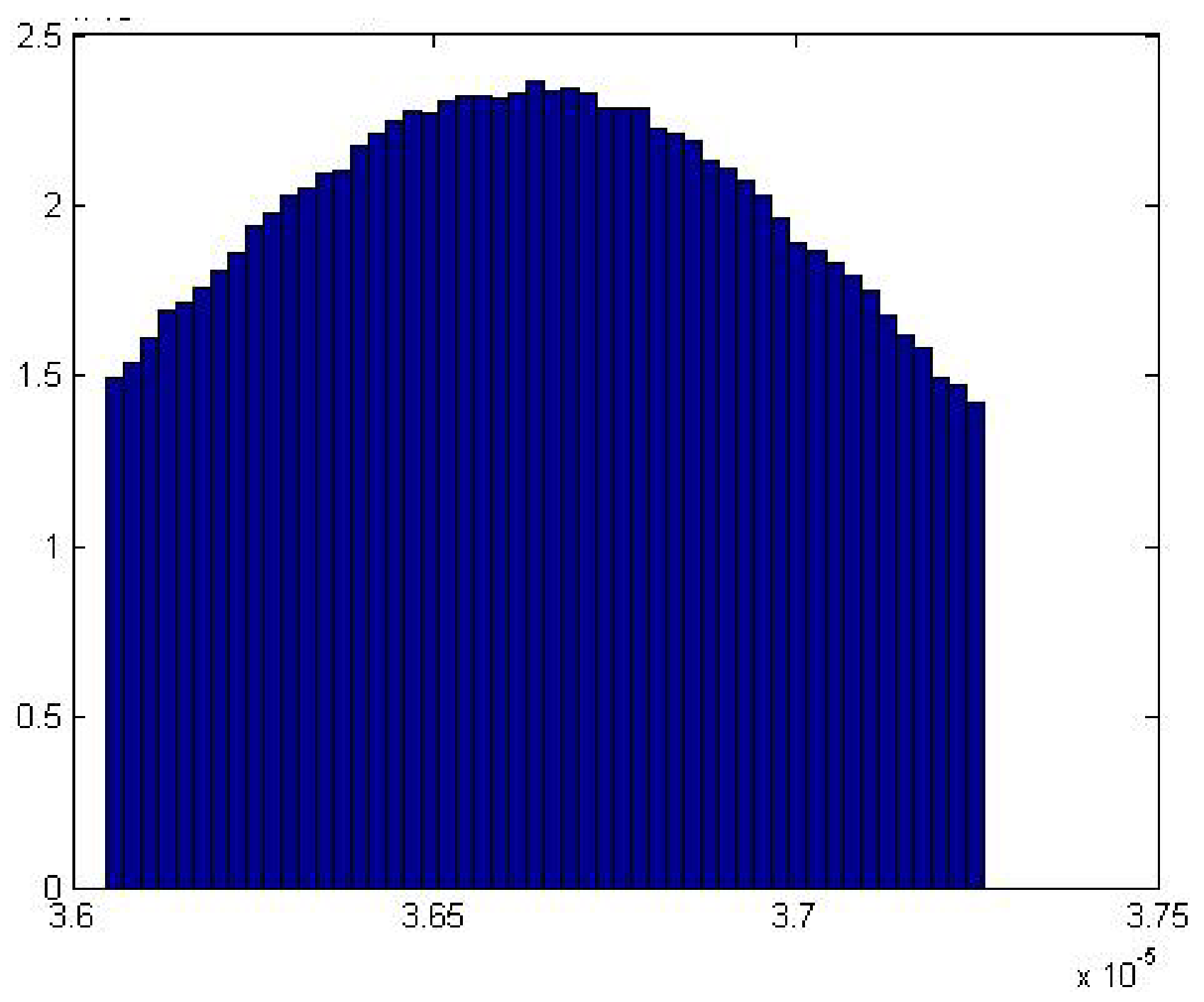

Figure 3. $X / Q$ values from random sampling.

The resulting dose consequence distribution is automatically analyzed to determine its similarity to well know distributions. If the resulting distribution is sufficiently similar to a well know distribution, the application provides information about the distributions such as the mean value and the probability that the dose will exceed a particular dose value.

Selecting the appropriate input parameter distribution is the most challenging aspect of performing a Monte Carlo based calculation of the potential dose. For example, Figure 4 shows the BR distribution for an adult male over a hypothetical 24 hour period with BRs for sleep, sedentary activity, light physical activity, and heavy physical activity (ICRP-89, "Basic Anatomical and Physiological Data for Use in Radiological Protection Reference Values"). The traditional approach suggested by DOE-STD-3009-2014, "Preparation of Nonreactor Nuclear Facility Documented Safety Analysis," is to select a value of $3.3 \times 10^{-2} \mathrm{~m}^{3} / \mathrm{hr}$, corresponding to light activity for an adult male. However, it is clear 
that there are periods where the BR is significantly lower and higher. SODA can randomly sample the distribution shown in Figure 4 when performing the dose calculation, thereby influencing the distribution of the resulting dose which helps inform decision makers by more clearly showing the range of potential dose result values.

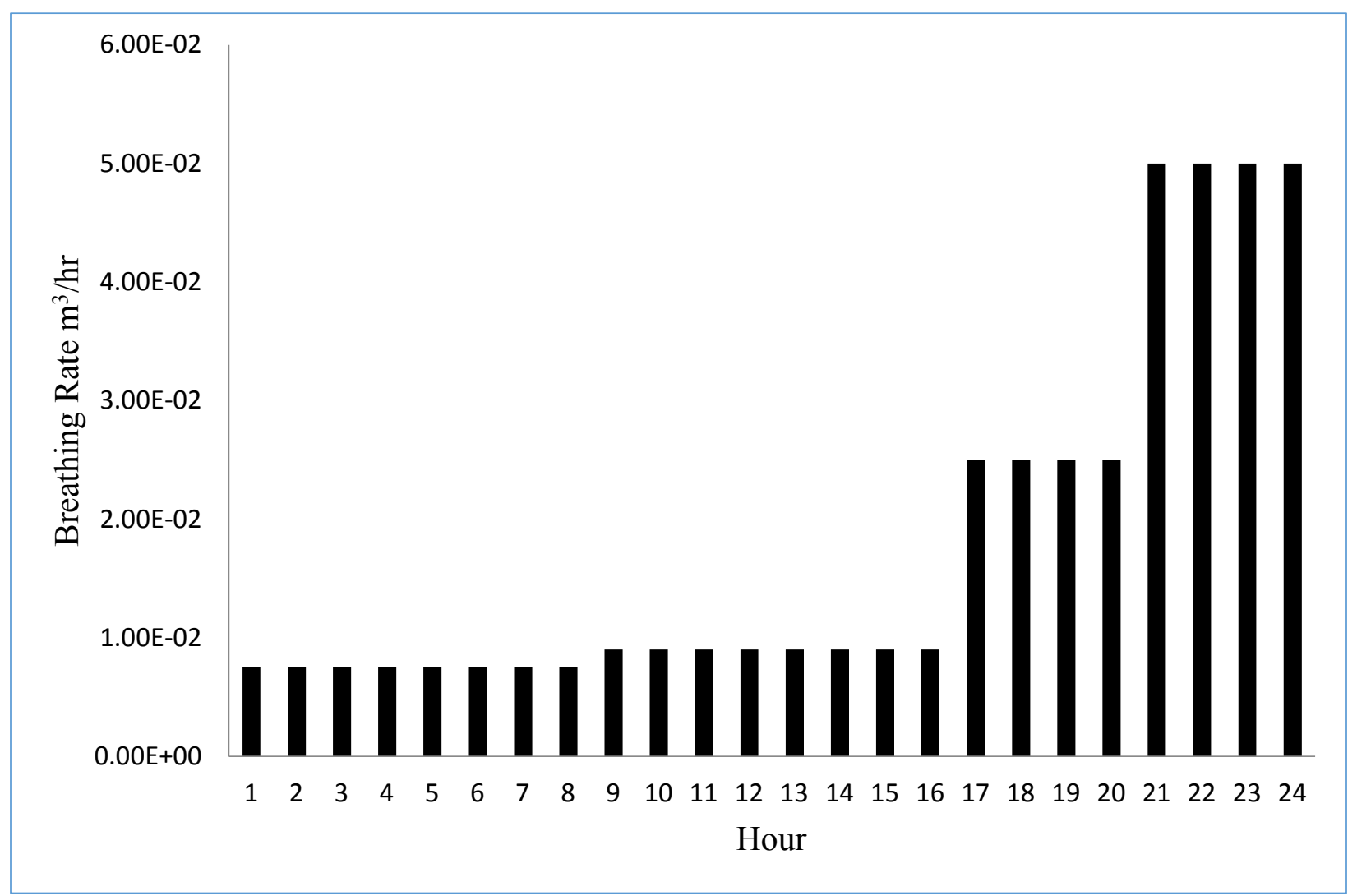

Figure 4. Adult male BR.

The SODA GUI interface screen is shown in Figure 5. The user can select the pre-defined distribution for each input variable along with the associated distribution key parameters, such as the mean value and variance. Individual input parameter sampled distribution plots can be requested. The user also selects the number of Monte Carlo calculations to execute. The resulting dose distribution is then displayed for the user. The code allows the user to save the input selections as well as export the resulting dose distribution plot. 


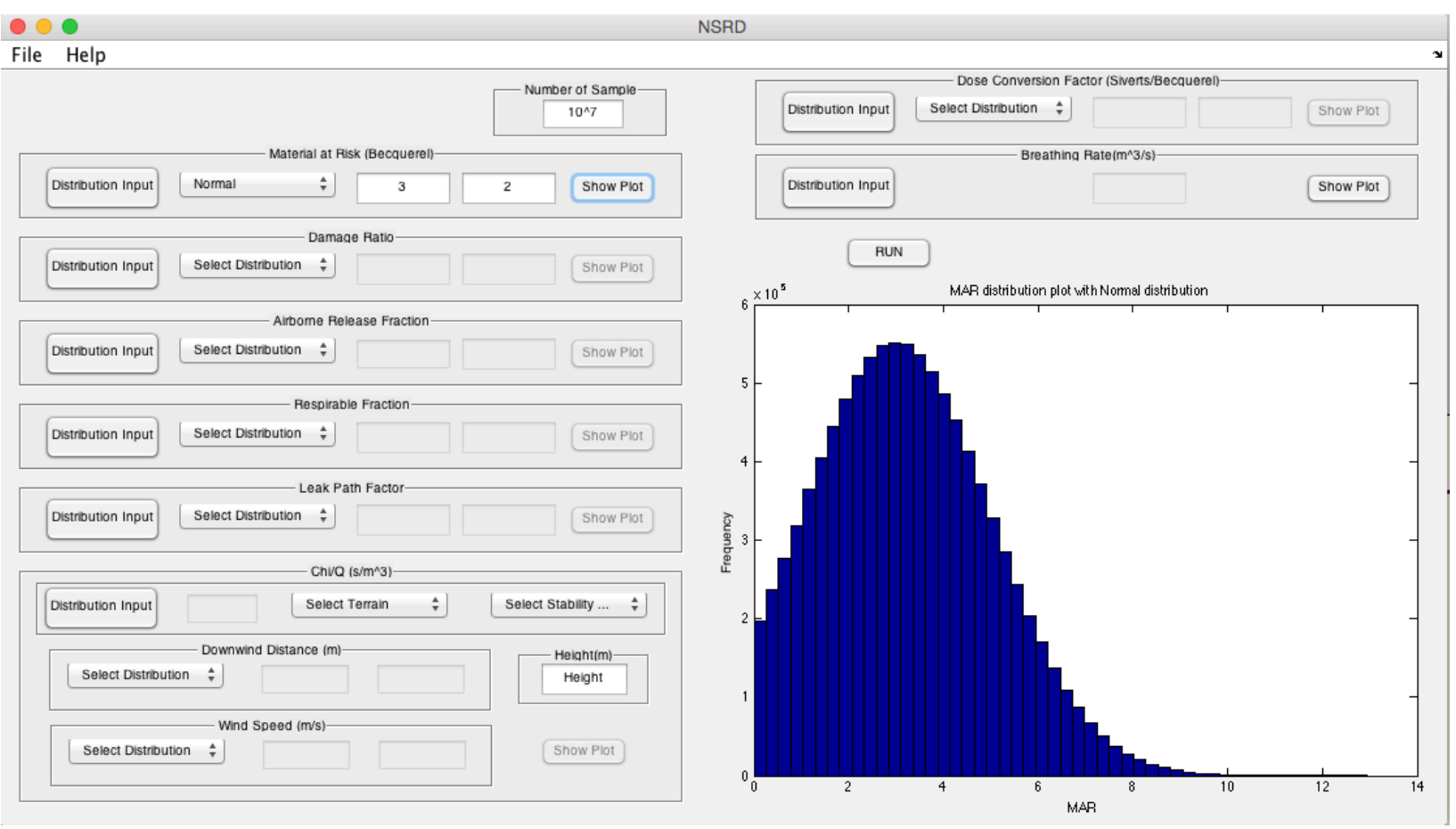

Figure 5. SODA GUI screen.

\subsection{Coding Framework}

The objective for this project was to develop a GUI based on intuitive functions. GUI applications are easy to use and require little memorization of command or syntax structure. As such, the SODA application needs little training to use. Help File instructions are provided to support rapid use. Furthermore, placing the mouse pointer over a button will display tips. The application was prepared in MATLAB, created by Math Works Inc. MATLAB contains a large standard library with easy to use integrated development environment (IDE). MATLAB is a high-level programming language; thus it is easy to code. For example, a simple addition of variables in MATLAB is straightforward whereas in lowlevel language, the user has to declare the variable type before doing any mathematical operations. High-level languages such as MATLAB are prevalent because they allow focus on the problem rather than the command syntax and code. With the advent of high speed processors, high-level languages are a good choice to develop prototype codes.

MATLAB was the choice for the application development because of the many built-in functions and libraries that come standard with the MATLAB package greatly reducing the time to create the application. Importantly, the MATLAB statistical toolbox as well as the MATLAB compiler toolbox was used to help develop the application. The statistical toolbox is used to fit probability distributions to data and generate random numbers for Monte Carlo simulations of various probability distributions. The 
MATLAB compiler toolbox is used for compilation of code to standalone application for Mac and Windows computers.

\subsection{GUI}

MATLAB utilizes the Graphical User Interface Development Environment (GUIDE). GUIDE was used to design SODA. Using the GUIDE layout editor, the user can graphically design the User Interface. GUIDE then automatically generates the MATLAB code for constructing the user interface. This allows the programmer to focus on the code that solves the problem rather than visual cosmetics of the application. GUIDE is accessible by typing guide in the MATLAB command window which activates the quick start menu shown in Figure 6.

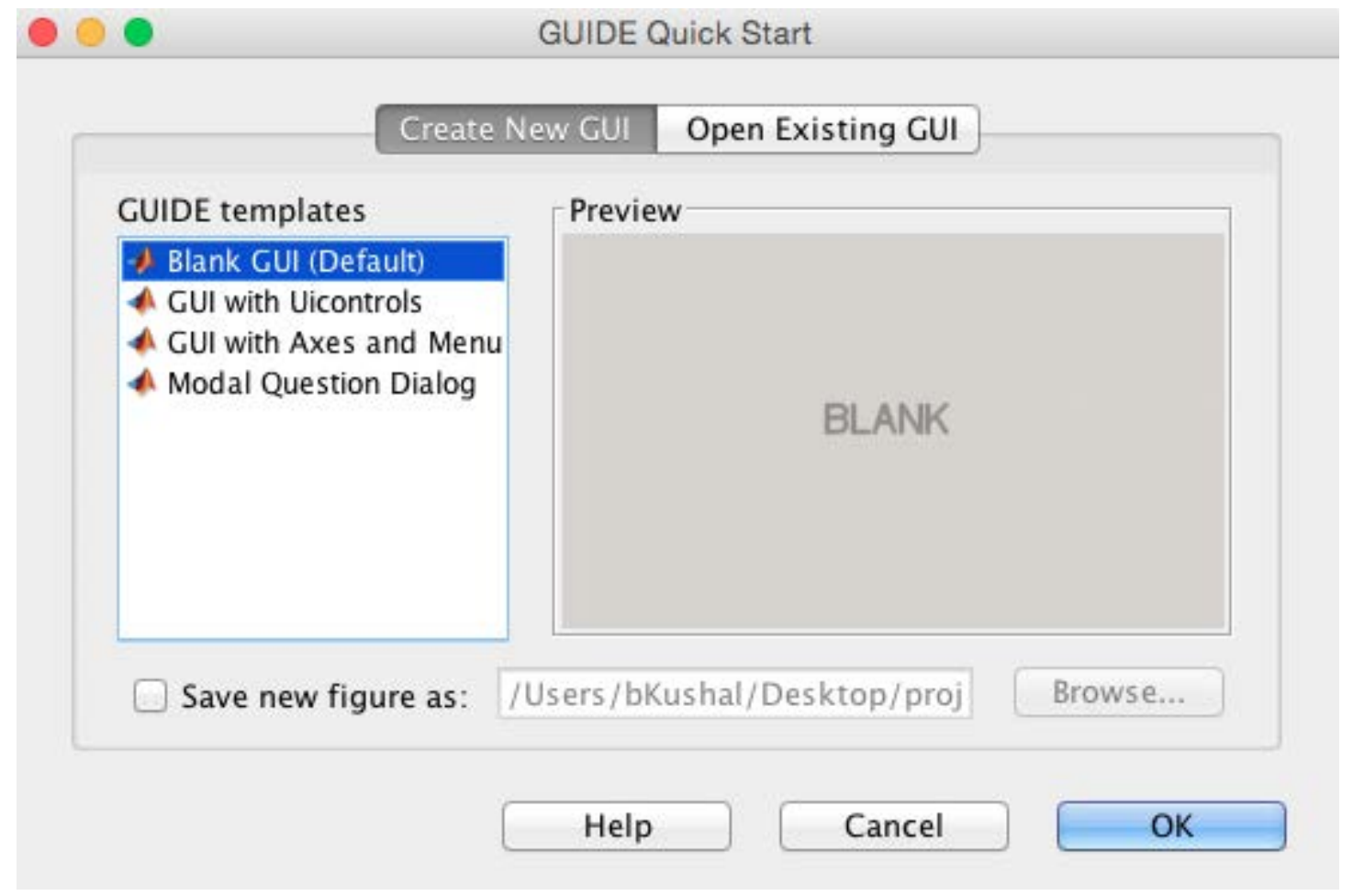

Figure 6. MATLAB GUI quick start.

The user has a choice to select from various GUI templates. To create SODA, a blank GUI (Default) was selected which reveals the window shown in Figure 7 where the user can drag and drop all the necessary tools required in the application. Here the user is creating the visual and cosmetic aspects of the application by deciding which tools are required for the application. 


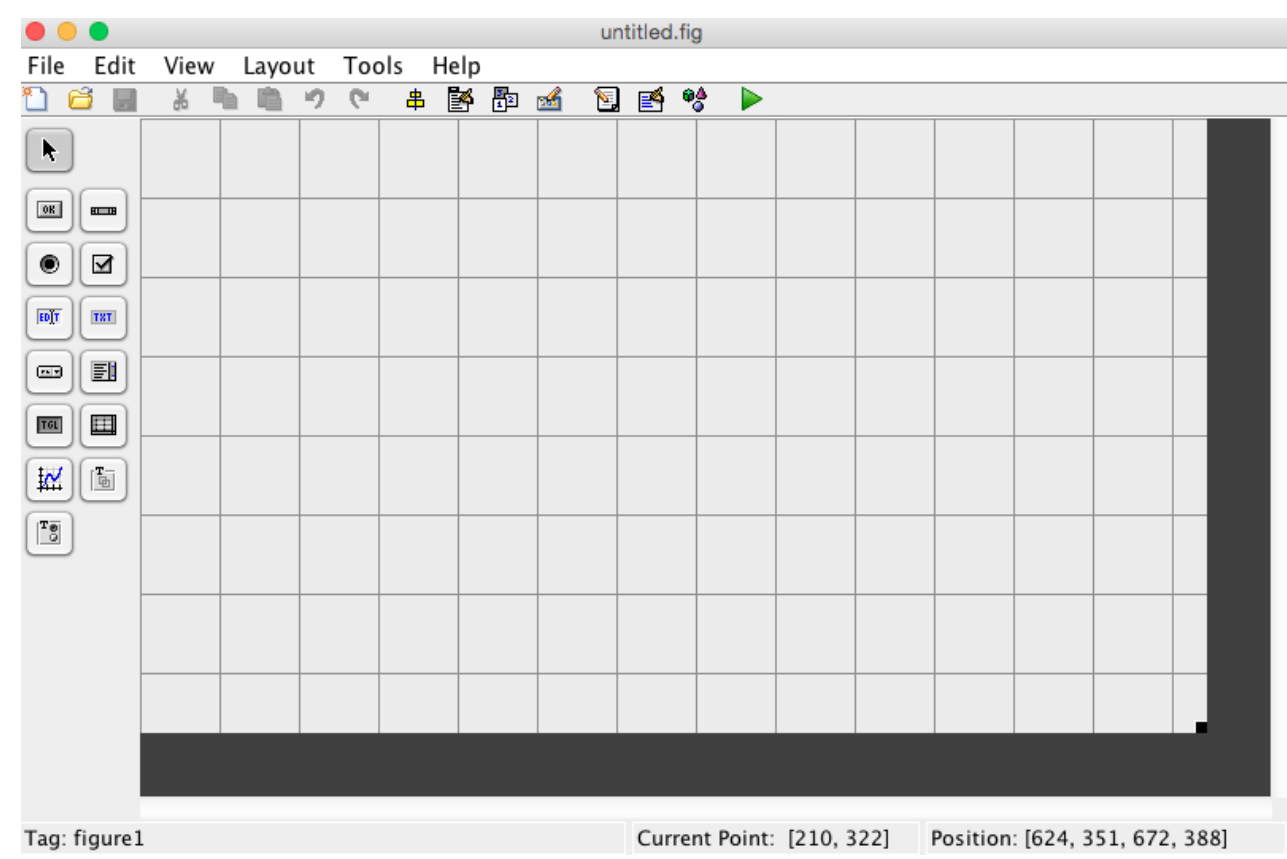

Figure 7. Default GUI figure window in MATLAB.

On the side panel shown in Figure 7, there are lists of tools that can be used in the layout window. These includes Push Button, Slider, Radio Button, Checkbox, Edit Text, Static Text, Pop-up Menu, List Box, Toggle Button, Table, Axes, Panel, and Button Group. In the SODA application, the Push Button, Edit Text, Static Text, Pop-up Menu, Toggle Button, Axes, and Panel tools were used. The SODA application uses two figures created using the GUI figure window: the Main Window and the About Window. First, the About Window shown in Figure 8 provides the logo of DOE and name of ISU, as well as static text identifying the names of the people involved in the project. 


\section{About}

\section{Idaho State \\ U NIVER S I T Y}

Dr. Chad Pope, P.E., Idaho State University Jason Andrus, Idaho National Lab

\section{Graduate Student}

Kushal Bhattarai, Idaho State Univeristy

Undergraduate Student

Abdullah Alomani

Abraham Chupp

Mason Jaussi

Tobi Okediran

Figure 8. SODA About window.

The main window, shown in Figure 9, uses the Edit Text option for user input. All user input on the SODA application is numeric. For example, $1.5^{*} 10^{\wedge} 5,1.5 \mathrm{E} 5,1.5 \mathrm{e} 5$ are acceptable inputs for the same value. The Panel option is used to group input parameters. The Toggle Button option is used to switch between single value input and distribution input. The Popup Menu option is used to select from the list of various probability distributions. The Push Button option makes it possible to execute code when the user presses those buttons. A Push Button is also used to run the program as well as to plot each parameter probability distribution plot. An Axes option is used to display the probability density curve (PDC) of parameters as well as compute the PDC of the CED. In addition, a push button is used to find the best fit for the resultant distribution of CED using the Bayesian Information Criterion (BIC). 

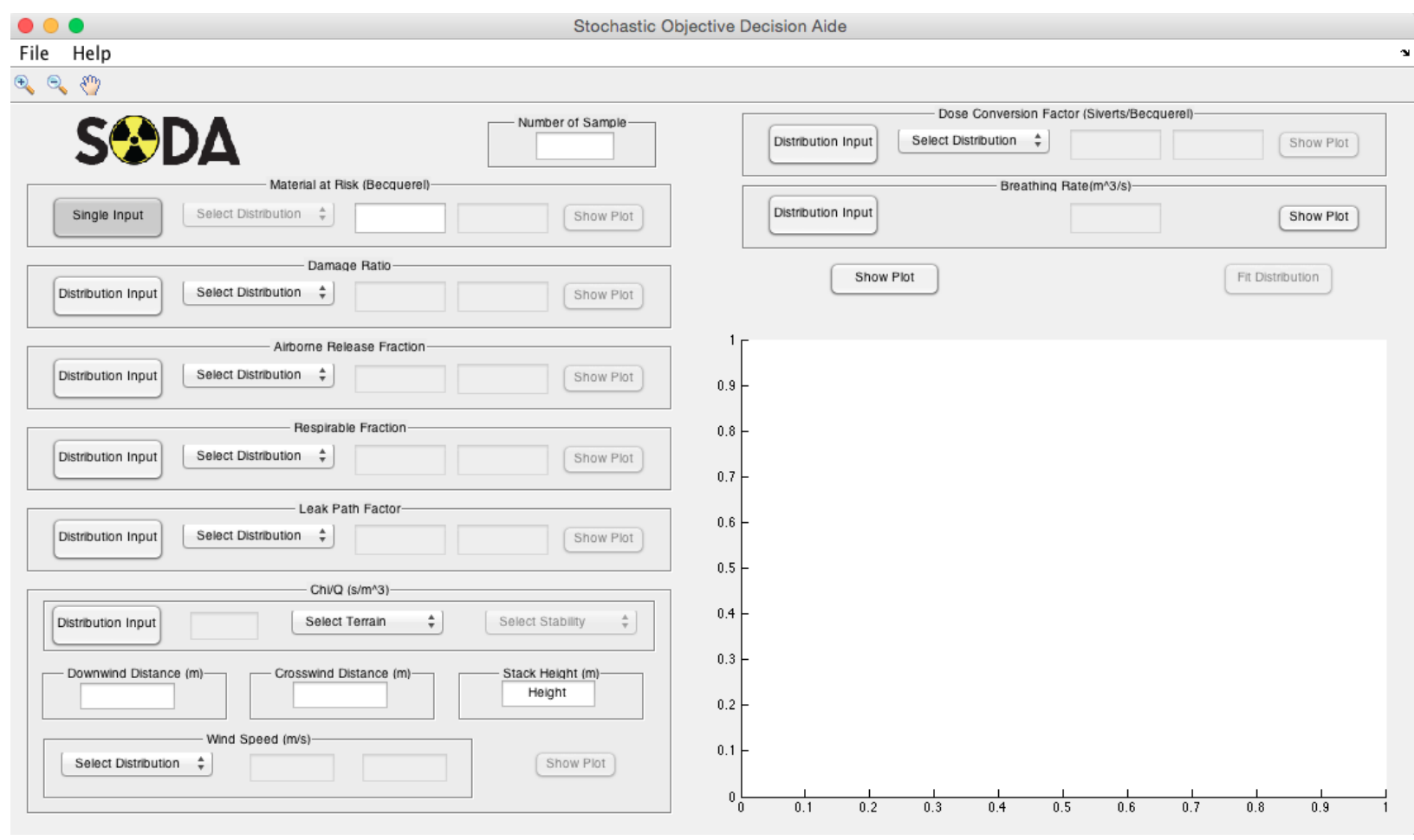

Figure 9. SODA main window.

The SODA application includes a menu and toolbar. In the toolbar there are three functions: zoom in, zoom out, and pan. In the main menu there are two options: File and Help. Each of them has a further detailed sub-menu. The File menu consists of five sub-menu options which are Load Workspace, Save Workspace, Save Image, Reset Random Number, and Exit. Load Workspace lets the user load a file which contains all the input parameters from a *.mat file. Save Workspace is used to save all the input parameters so that the user does not have to re-type the input data to run the program. Save Image will let the user save the plot image into a *.jpg, *.tif, or * .png image file. Reset Random Number menu will reset the Mersenne Twister random number algorithm so that calculations can be performed using different computers (or repeated) to get same result. The Exit menu will let the user exit the application with a confirmation to make sure that the user is about to exit the application.

\subsection{Monte Carlo Method}

SODA uses Monte Carlo techniques in which the application uses repeated random sampling to compute the CED distribution. SODA can handle up to $10^{8}$ samples depending on the available computer memory. For each input parameter, the user can select from four different probability distributions: Normal, Uniform, Beta, and Exponential. The user can verify the resulting input parameter distribution by clicking the show plot button for each parameter. Based on the distribution selection, 
different user input values are required. For a normal distribution, the user is required to input the mean and standard deviation. For the Beta distribution, the user is required to input the alpha and beta parameter. For a uniform distribution, the user is required to input the upper and lower limit. Finally, for the exponential distribution, the user is required to input the mean. Once the user provides the input parameter values, SODA creates a string of random numbers to sample the specified distributions.

\subsection{Distribution Mathematics}

The normal distribution, also known as Gaussian distribution, is a common distribution in which the probability density function (pdf) has a bell shaped curve. The pdf equation of a normal distribution is:

$$
f(x \mid \mu, \sigma)=\frac{1}{\sigma \sqrt{2 \pi}} e^{-\frac{(x-\mu)^{2}}{2 \sigma^{2}}}
$$

where $\mathrm{mu}$ is the mean or expected value, and sigma is standard deviation. Examples of normal distributions using different mean and standard deviation values are provided in Figure 10.

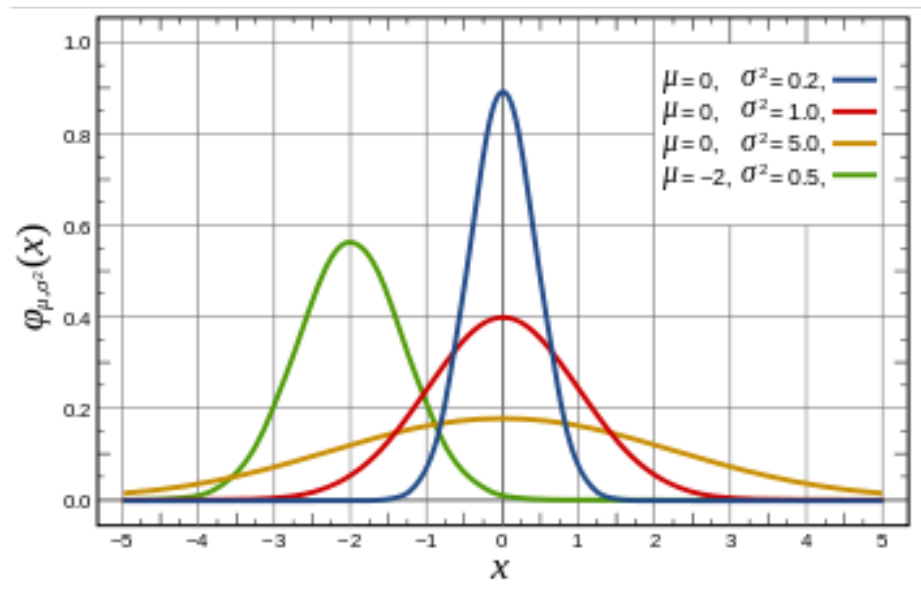

Figure 10. Normal distributions.

The beta distribution, plotted in Figure 11, is defined over the interval of zero to one with two positive shape parameters, alpha and beta. The pdf equation of a beta distribution is:

$$
\frac{x^{\alpha-1}(1-x)^{\beta-1}}{\mathrm{~B}(\alpha, \beta)}
$$

where B is beta function. Since beta distribution is defined in between zero and one, it can be used for damage ratio and LPF. 


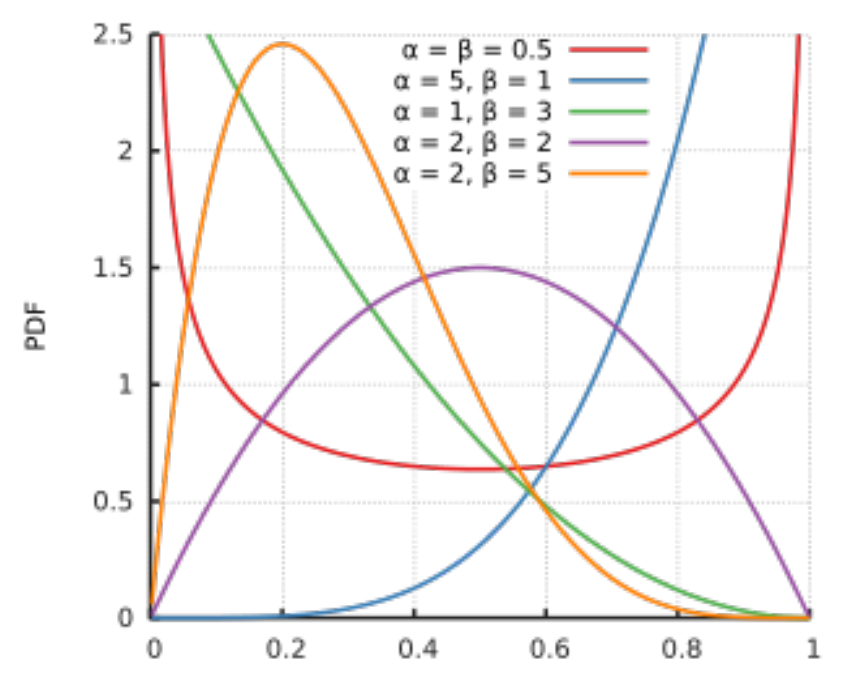

Figure 11. Beta distribution.

The uniform distribution is uniform, continuous distribution in which all possible values between the minimum and maximum value are equally probable. The pdf equation of a uniform distribution is:

$$
\begin{cases}\frac{1}{b-a} & \text { for } x \in[a, b] \\ 0 & \text { otherwise }\end{cases}
$$

where $b$ is the maximum value and $a$ is minimum value. A uniform distribution plot is shown in Figure 12.

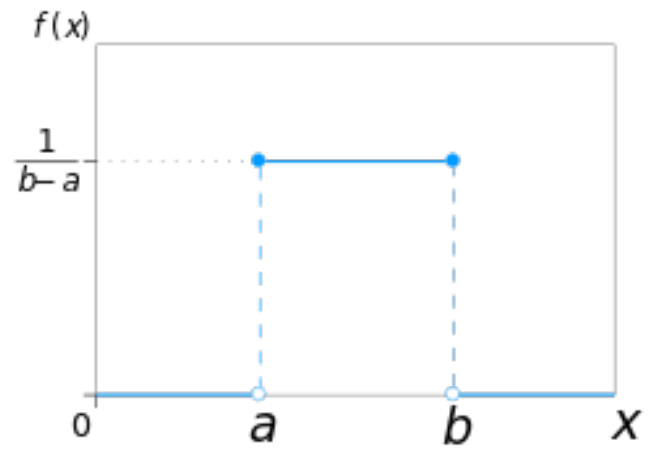

Figure 12. Uniform distribution.

The exponential distribution describes a process in which events occur continuously and independently at a constant average rate lambda (see Figure 13). The exponential distribution equation is: 


$$
f(x ; \lambda)= \begin{cases}\lambda e^{-\lambda x} & x \geq 0 \\ 0 & x<0\end{cases}
$$

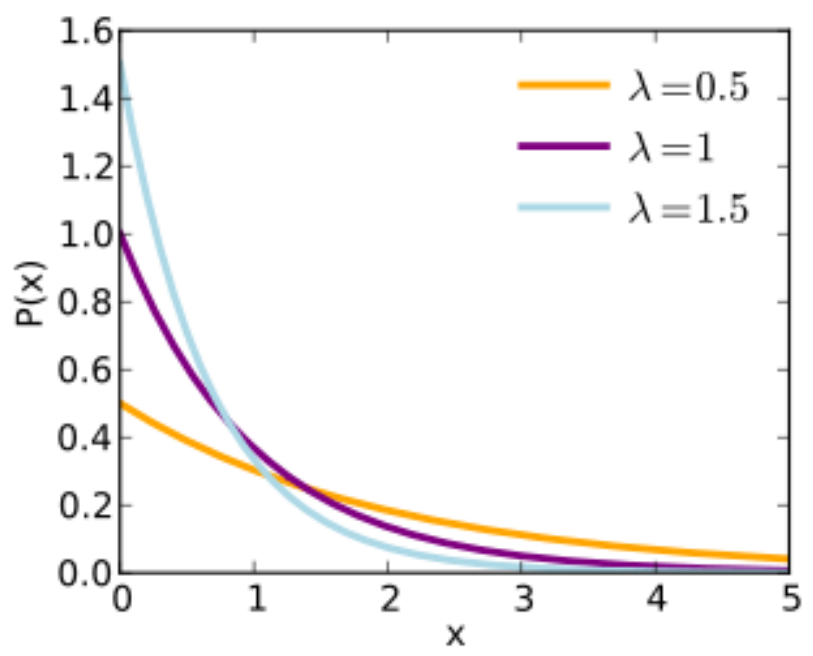

Figure 13. Exponential distribution.

In addition to the distributions described above, a specialized BR distribution is available in the application. The distribution is intended to be reasonable and yet conservative. It is applicable for scenarios where the event can occur at any time throughout a 24-hour period. A 24-hour period was used with an adult male BR consisting of eight hours per day resting, eight hours per day sitting, four hours per day of light exercise, and four hours per day of heavy exercise. The distribution is sampled by scaling the random number generator and selecting the BR reflected in Table 1, which uses data from ICRP-89.

Table 1. Breathing rate data.

\begin{tabular}{|c|c|}
\hline Status & Breathing rate $\left(\mathrm{m}^{3} / \mathrm{s}\right)$ \\
\hline Resting & $1.25 \mathrm{e}-4$ \\
\hline Sitting & $1.5 \mathrm{e}-4$ \\
\hline Light exercise & $4.17 \mathrm{e}-4$ \\
\hline Heavy exercise & $8.33 \mathrm{e}-4$ \\
\hline
\end{tabular}

The resting BR and sitting BR are sufficiently similar to allow using the sitting BR for both. The sampling strategy results in the $1.5 \mathrm{e}-4$ value being selected $66 \%$ of the time, and the light exercise value, 
$4.17 \mathrm{e}-4$, and the heavy exercise vale, $8.33 \mathrm{e}-4$, each being selected $17 \%$ of the time. The resulting BR distribution is shown in Figure 14.

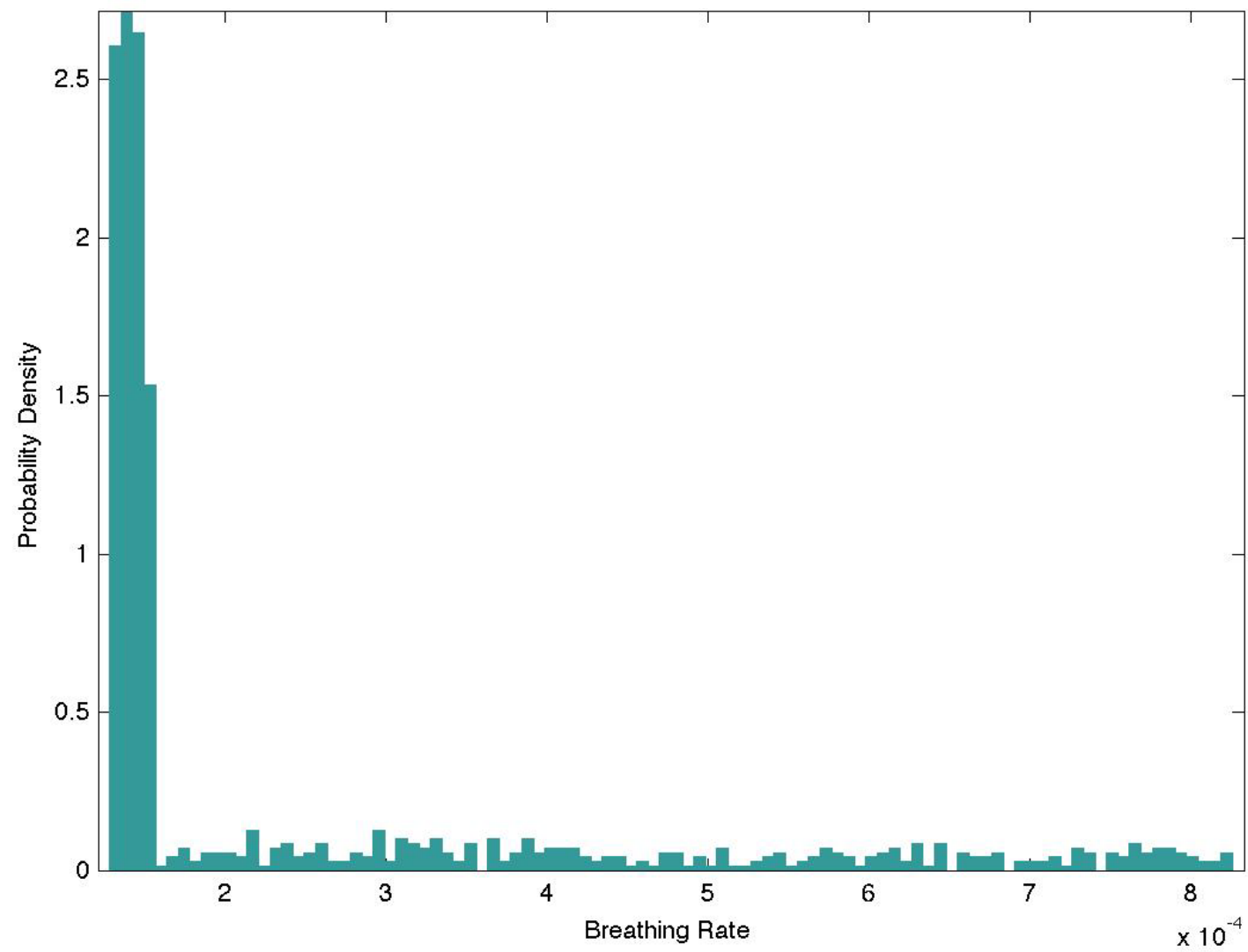

Figure 14. Breathing rate distribution.

A simplified damage ratio experiment was performed to support a rudimentary estimate for the damage ratio distribution that could be applied to certain scenarios. The experiment investigated the damage ratio for dropped radioactive material containers. The damage ratio experiment involved both $1-\mathrm{m}$ and 3-m drop tests onto a concrete surface. The containers involved in the experiment were one pint capacity and one quart capacity. The containers used press fit lids similar to paint cans. Each container was filled 3/4 full with rock salt to simulate radioactive material. Twelve containers of each capacity were used in the experiment. The one pint capacity containers were initially dropped from the 1-m height. A total of 100 drop tests with one pint capacity containers was performed from the 1-m height. After each container was dropped, it was visually examined to qualitatively determine if the container had breached. Containers that were not breached were subjected to additional drop testing. Containers that breached were photographed, the amount of salt that escaped from the container was quantified, the salt was returned to the container, the lid was reinstalled, and the container was reused for subsequent drop testing. 
A similar approach was used for the 3-m drop test. The 3-m drop test involved both the one pint capacity containers and the one quart capacity containers. A total of 300 container drop test were performed. No container failure mode other than lid failure was observed. Approximately $20 \%$ of the container drops resulted in damage sufficient to allow release of radioactive material.

The drop testing experiment provides a rudimentary understanding of the likelihood of container breaching due to a drop event which can help understand the appropriate damage ratio distribution to select for a dose consequence calculation associated with a particular accident scenario. Since the containers used in the experiment have no quality assurance pedigree and are very thin walled, the experiment is intended to provide some rudimentary insight into a reasonable, but conservative, damage ratio distribution for accident scenarios involving multiple radioactive material containers. Appendix D contains the drop test results data.

\subsection{Bayesian Information Criterion}

Bayesian Information Criterion is the method by which SODA finds the best possible probability distribution for the resultant CED distribution. BIC value is computed using a likelihood function of the estimated model. A likelihood function is the probability or probability density for the occurrence of a sample configuration $x 1, x 2, x 3, \ldots, x N$ given that the probability density $f(x$, alpha) with parameter alpha is known. The smaller the BIC value indicates a better model fit. Figure 15 below provides an example of a BIC fit. 


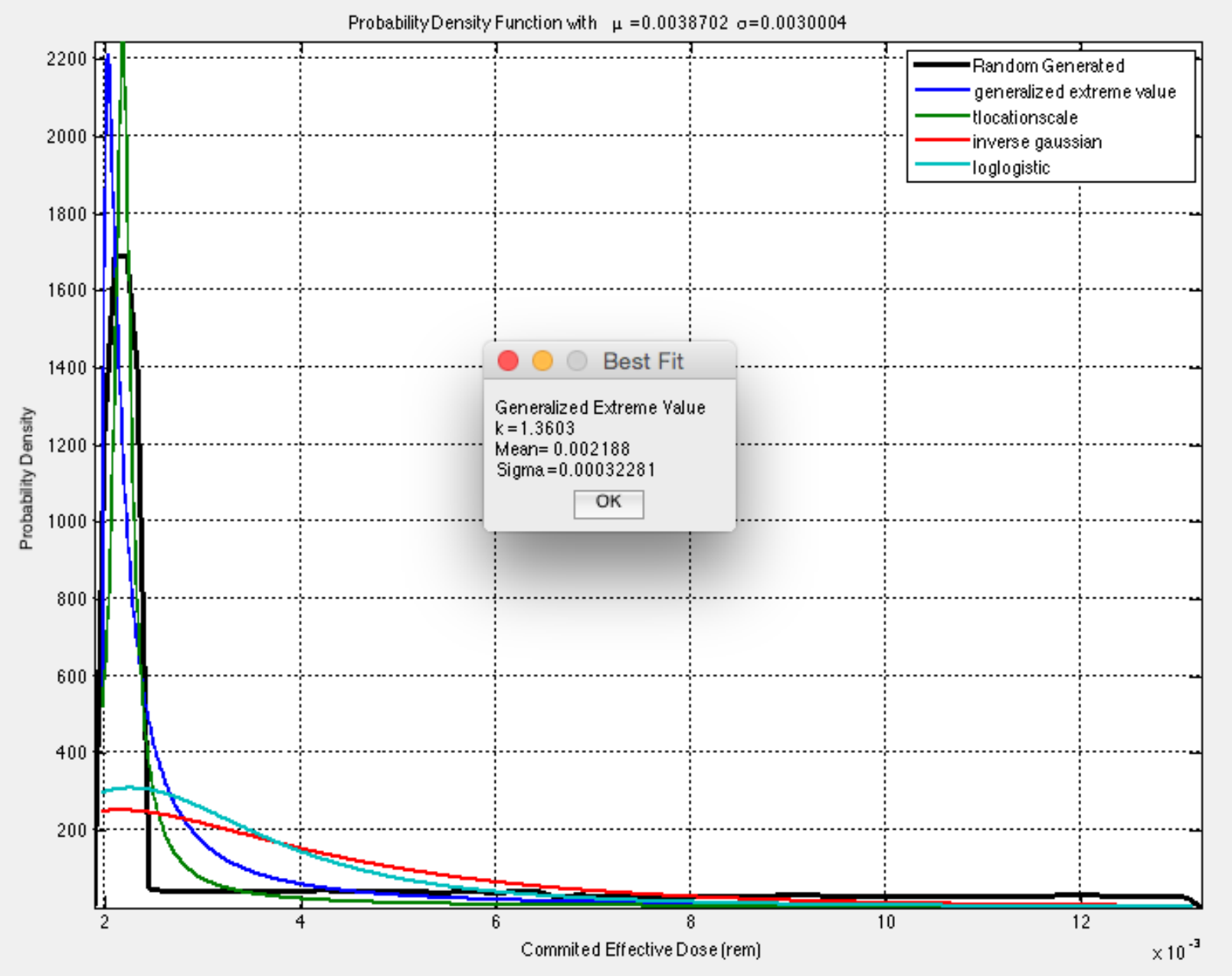

Figure 15. Example grapnhical representation of BIC fit to a randomly generated dose distribution.

\section{COMPARISON WITH RSAC}

Numerous improvements were identified during beta testing conducted by undergraduate students. Verification calculations were also performed to ensure that SODA was performing properly. The application was verified by comparing results obtained using RSAC 7.2.0 calculations for a simplified scenario involving the release of $1 \mathrm{Ci}$ of Pu-239. Fixed parameters used for the verification case are provided in table $x$ below. Results obtained from RSAC, SODA, and a hand calculation are provided in Table y and show good agreement using fixed parameters in SODA. 
Table 2. Verification case input data.

\begin{tabular}{|l|c|}
\hline \multicolumn{1}{|c|}{ Parameter } & Value \\
\hline ARF & $2.00 \mathrm{e}-3$ \\
\hline RF & 0.3 \\
\hline $\begin{array}{l}\text { Wind Speed, mixing height, release } \\
\text { height }\end{array}$ & $1 \mathrm{~m} / \mathrm{s}, 400 \mathrm{~m}, 0 \mathrm{~m}$ \\
\hline Pasquill Class & $\mathrm{F}$ \\
\hline Meteorology & Hillsmier-Gifford Sigmas \\
\hline Downwind distance & $5000 \mathrm{~m}$ \\
\hline Calculated X/Q & $5.925 \mathrm{E}-5$ \\
\hline Breathing Rate & $3.33 \mathrm{E}-04 \mathrm{~m}^{3} / \mathrm{sec}$ \\
\hline Pu - DCF Clearance Class & $\mathrm{F}$ \\
\hline
\end{tabular}

Table 3. Verification results comparison.

\begin{tabular}{|c|c|c|c|}
\hline & RSAC 7.2.0 & SODA & Hand calculation \\
\hline $\begin{array}{c}\text { Total CED } \\
\text { (rem) }\end{array}$ & $5.26 \mathrm{E}-3$ & $5.256 \mathrm{E}-3$ & $5.256 \mathrm{E}-3$ \\
\hline
\end{tabular}

\section{CONCLUSION}

A Monte Carlo based code system has been developed to stochastically analyze radiological material release scenarios providing potential dose distribution results. The computer code, named Stochastic Objective Decision-Aide, provides a portable and simple to use tool to better inform decision making associated with establishing nuclear facility MAR limits and safety SSC selection. Traditional radioactive material release modeling codes typically provide a bounding single point estimate of receptor dose. While this approach attempts to bound the dose estimate, it falls short in providing quantification of the expected value and the uncertainty associated with the dose estimate. This is particularly problematic when one considers the lack of governing distribution identification for input parameters. Thus, decisions regarding potential doses are frequently overstated, leading to excessively conservative MAR limits and potentially over selection of safety SSCs. 
Rather than producing a point estimate of the dose, SODA produces a dose distribution result to allow a deeper understanding of the dose potential. SODA allows users to select the distribution type and parameter values for all of the input variables used to perform the dose calculation. SODA then randomly samples each distribution input variable and calculates the overall resulting dose distribution. In cases were an input variable distribution is unknown, a traditional single point value can be used. SODA was developed using the MATLAB coding framework. The software application has a graphical user input. SODA can be installed on both Windows and Mac computers and does not require MATLAB to function.

It is important to note that SODA does not replace or compete with codes such as MACCS or RSAC; rather it is viewed as an easy to use supplemental tool to help improve risk understanding and support better informed decisions.

To further magnify SODA, it is proposed that the code be expanded to allow more detailed MAR selection, incorporate user defined input variable distributions, perform a comprehensive parametric study, and complete code verification and validation. Systematic investigation of each input parameter contributing to the dose result can be pursued to quantify the parameter's contribution to the overall dose estimate uncertainty. Systematic study of each contributing parameter will lead to identification of the parameters that have the largest impact on the resulting dose estimate uncertainty. Once identified, investigation into reducing uncertainty in the key parameters can be accomplished. 


\section{Appendix A: \\ SODA Help File Information}

Provided below is a listing of the help file topics that are linked within SODA to assist users with various issues. Following the summary table, the actual images that are included in the SODA help file are provided.

\section{SODA Help File Summary}

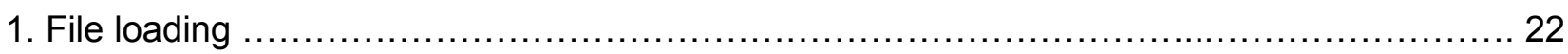

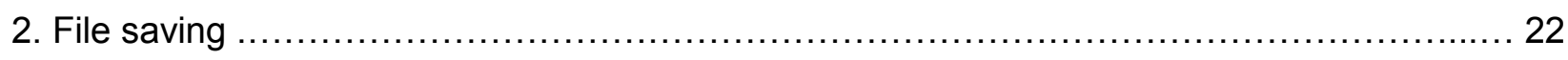

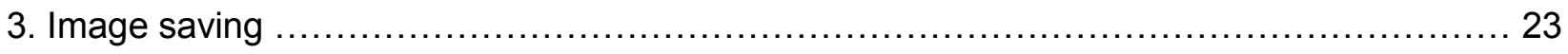

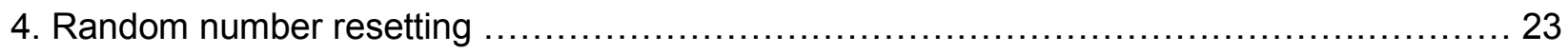

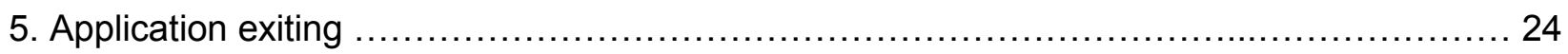

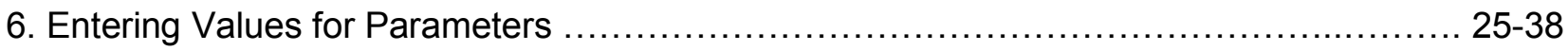

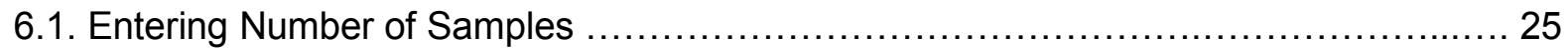

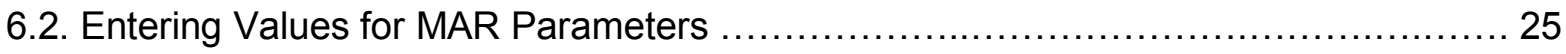

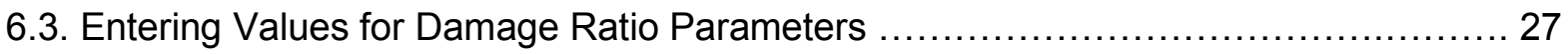

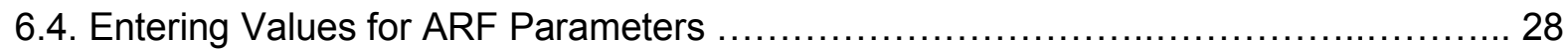

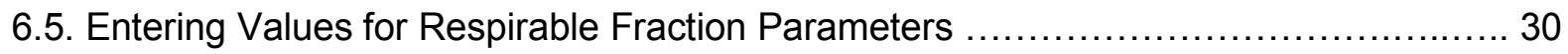

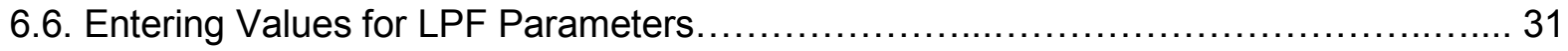

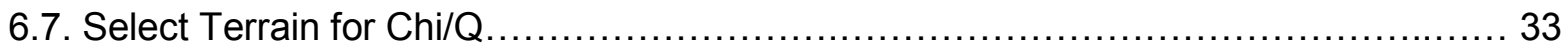

6.8. Entering Values for Downwind Distance, Crosswind Distance, and Stack Height ....... 34

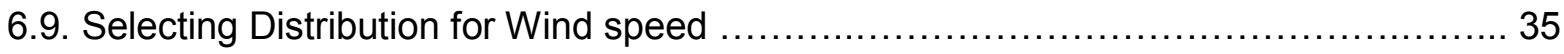

6.10. Entering Values for Dose Conversion Factor Parameters ....................... 35

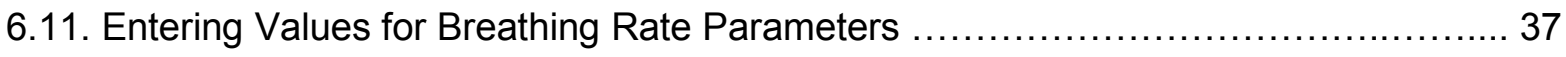


INL/EXT-15-36669

Rev. 0 September 2015

Page 22 of 129

\section{A.1. File Loading}

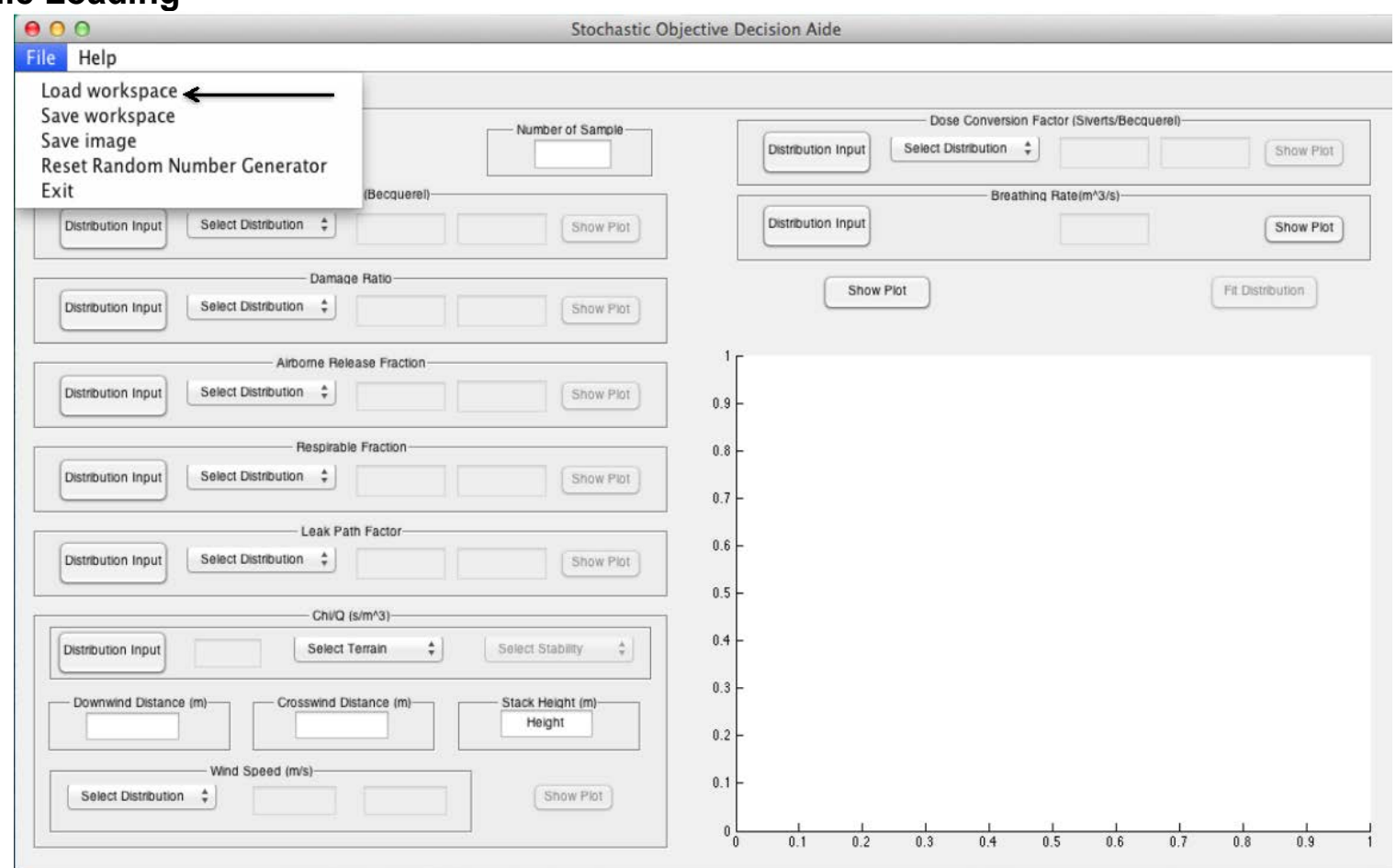

To load a file, go to "file", then "Load workspace"

\section{A.2. File Saving}

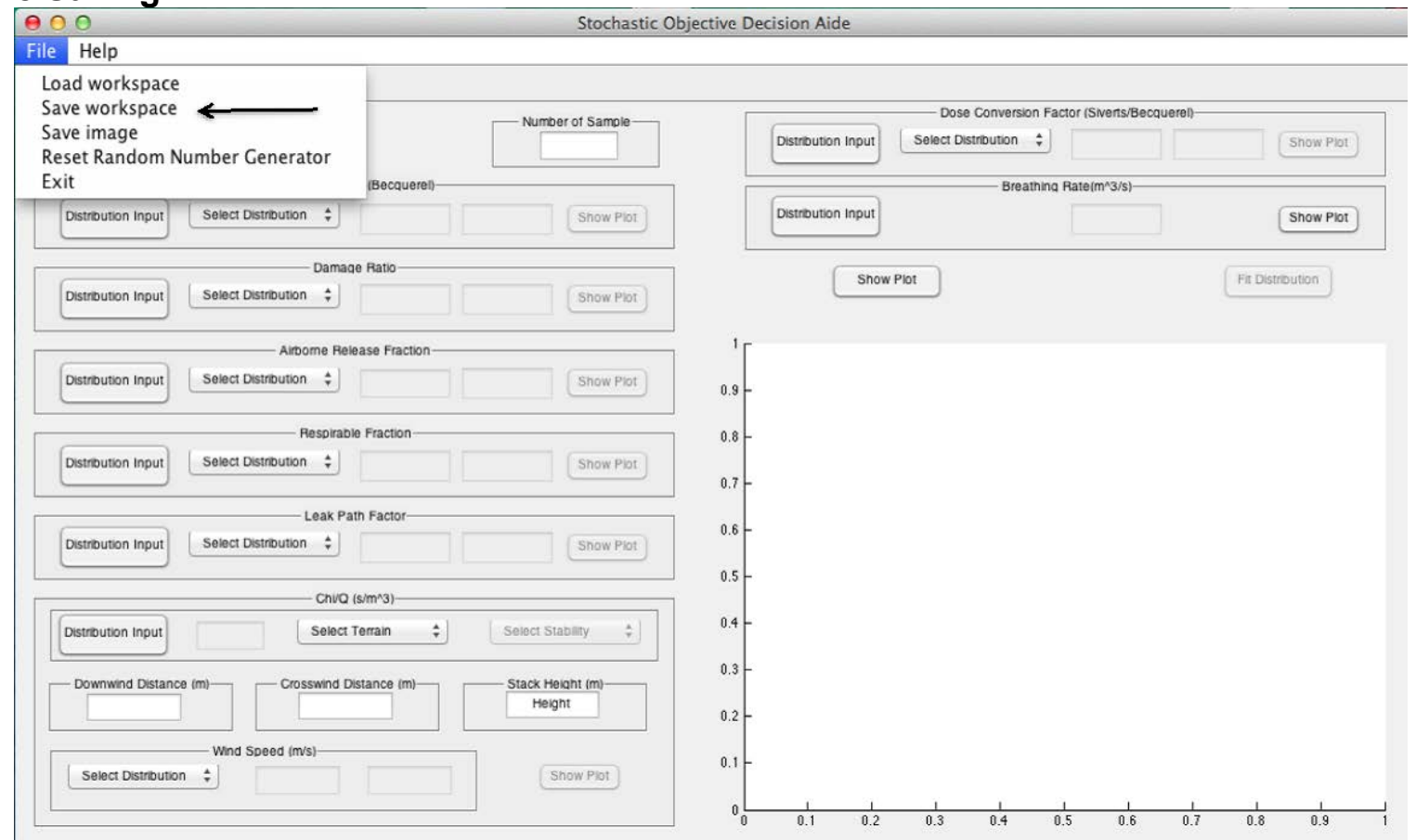

To save a file, go to "file", then "Save workplace". 
INL/EXT-15-36669

Rev. 0 September 2015

Page 23 of 129

\section{A.3. Image Saving}

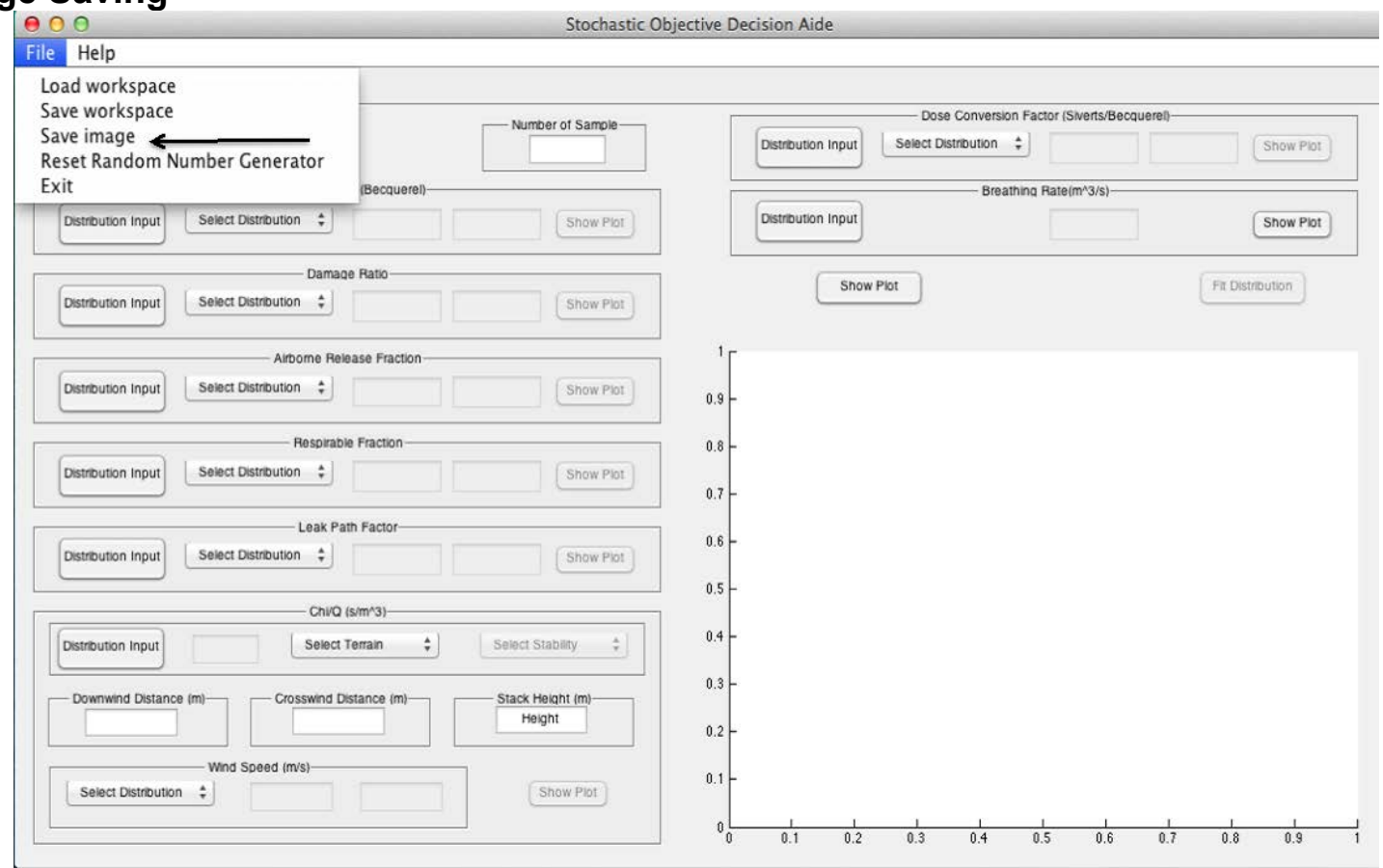

To save an image, go to "file", then "Save workplace".

\section{A.4. Resetting Random Number Generator}

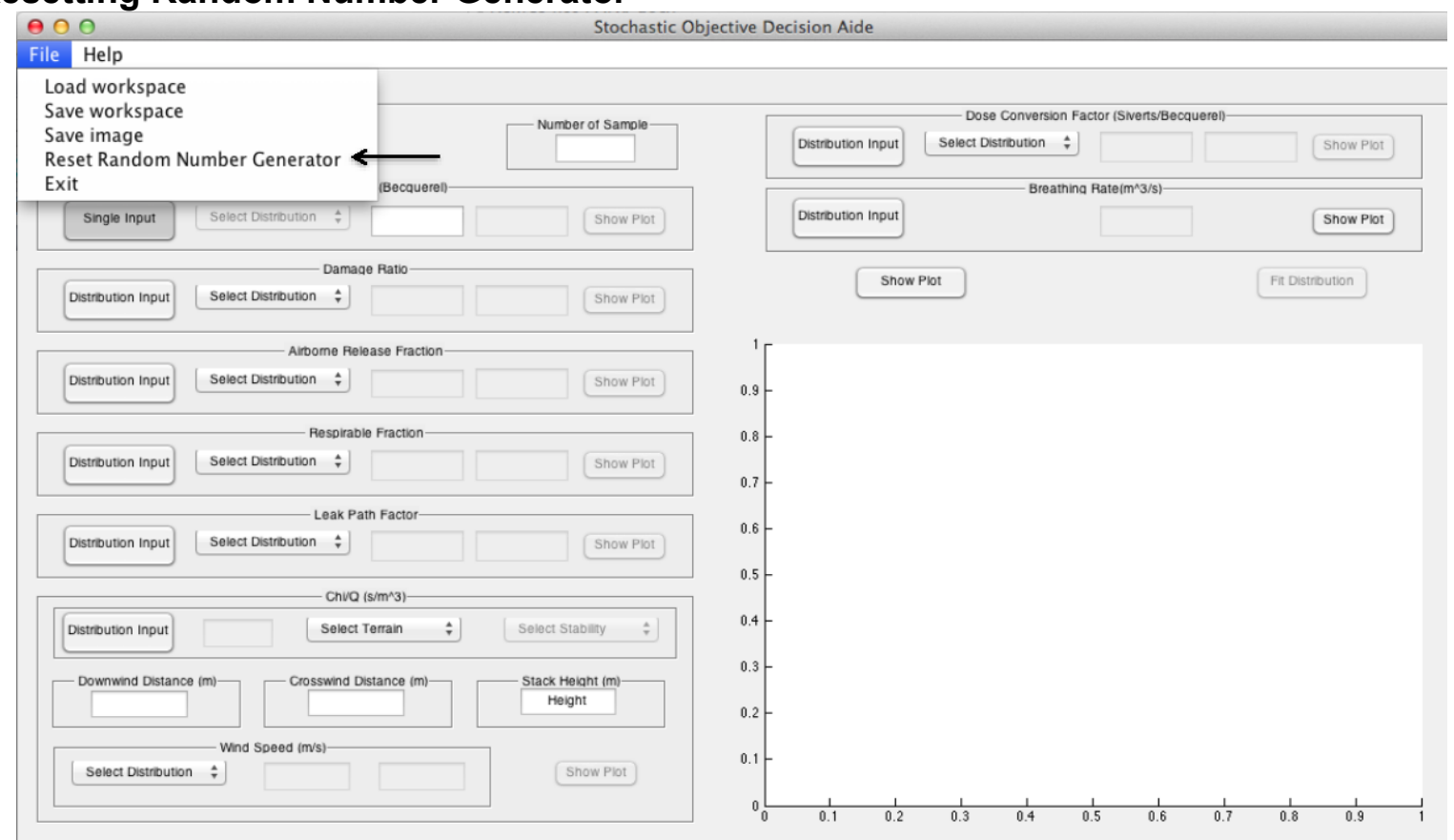

To reset the Mersenne Twister random number algorithms so that the calculations can be performed in two machines or repeated to get same result, click on "Reset Random Number Generator". 
INL/EXT-15-36669

Rev. 0 September 2015

Page 24 of 129

\section{A.5. Exiting the Program}

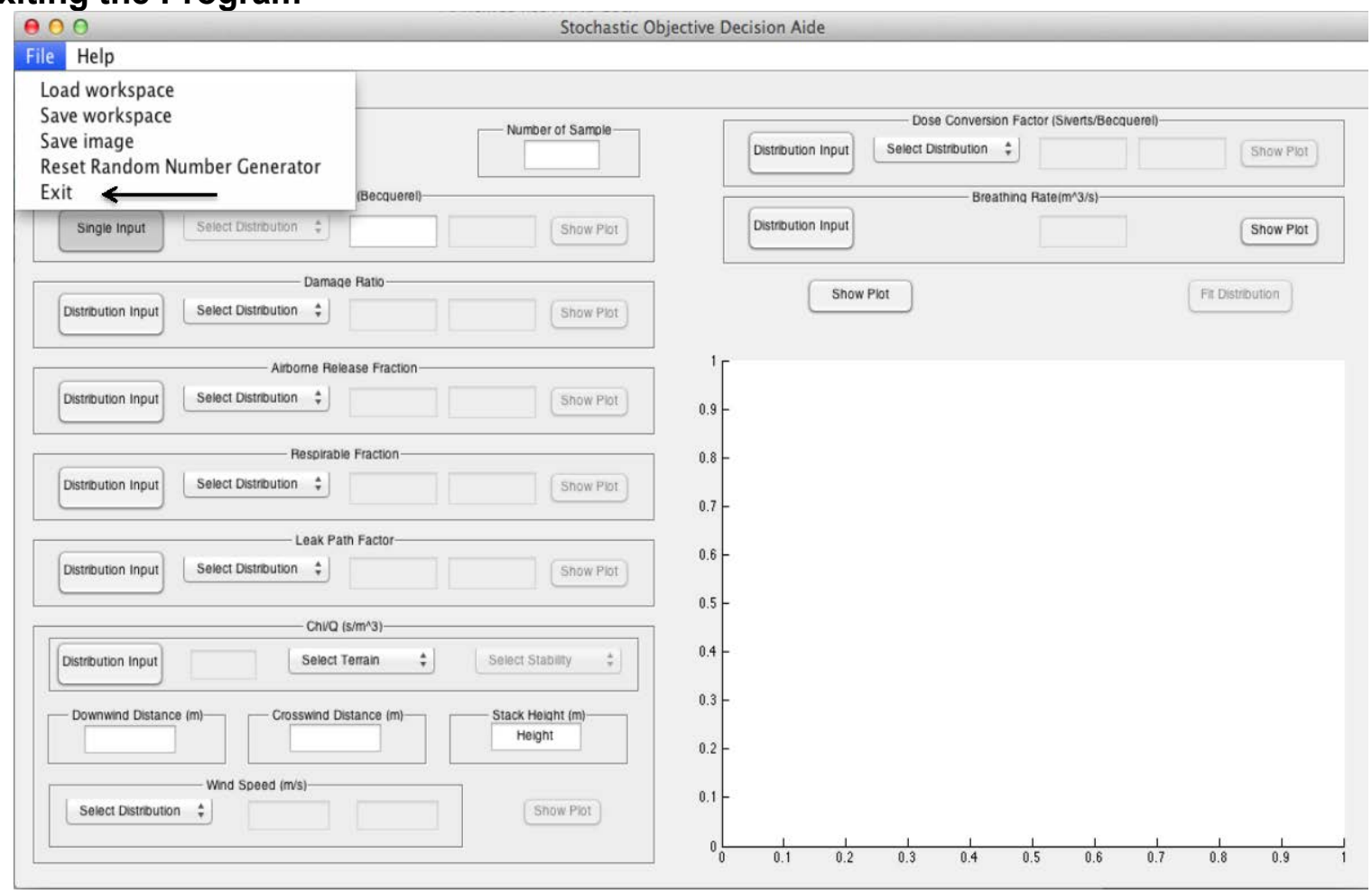

To exit the application, go to "file", then "Exit". 


\section{A.6. Entering Values for Parameters}

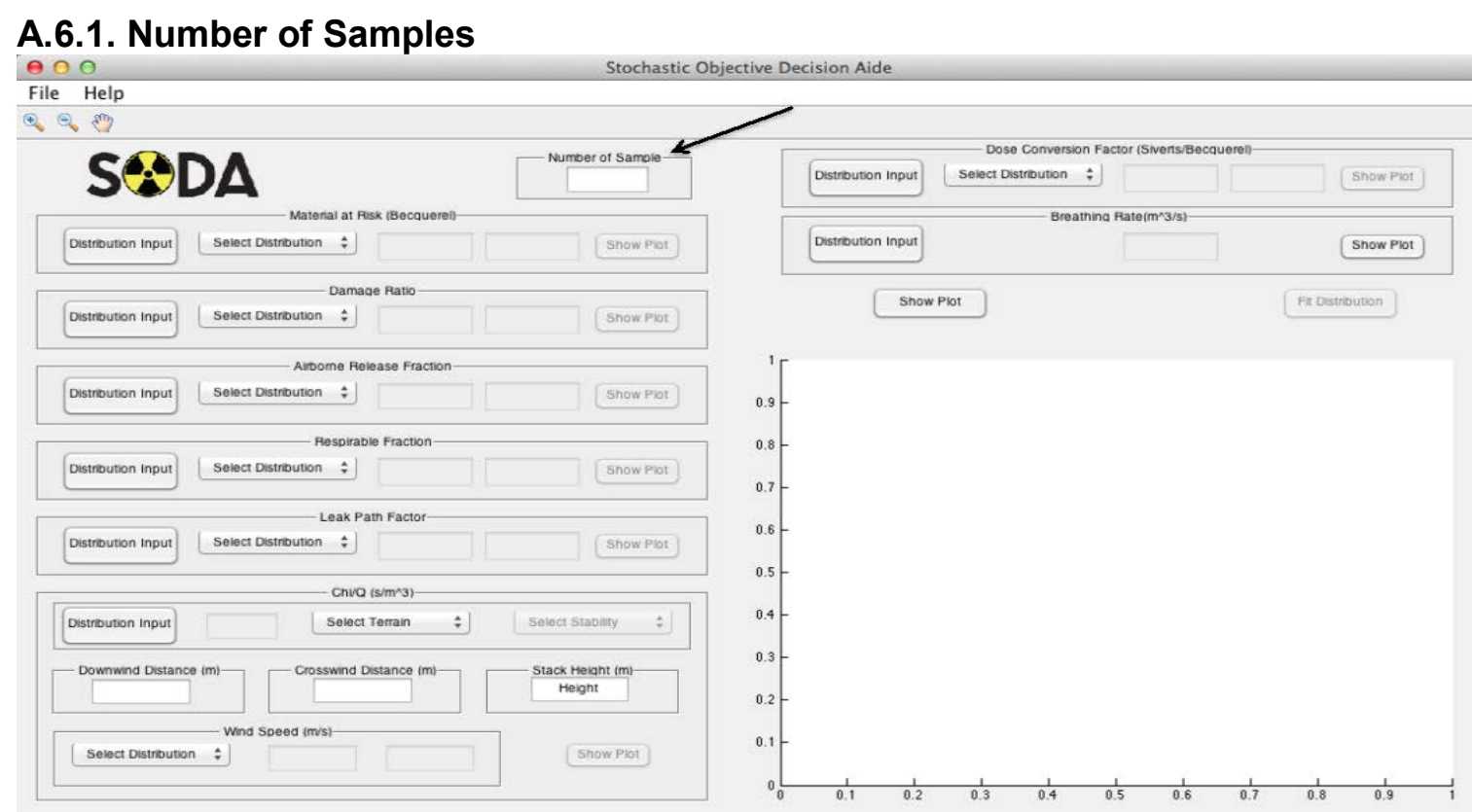

Enter the Number of samples.

\section{A.6.2. Entering Values for MAR Parameters}
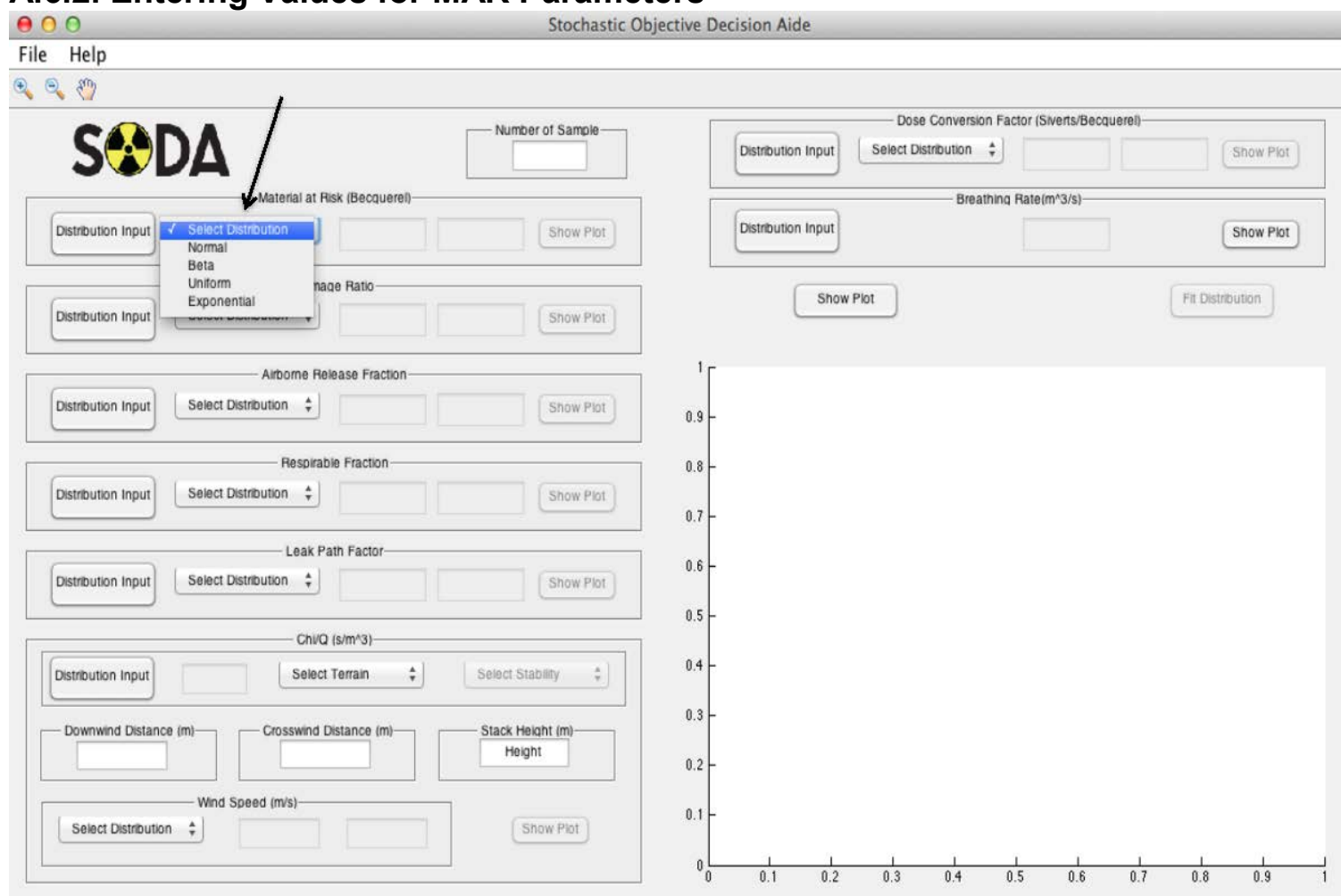

In Material at risk, for a distribution input, choose the distribution type (Normal, Beta, Uniform, Exponential) 
INL/EXT-15-36669

Rev. 0 September 2015

Page 26 of 129

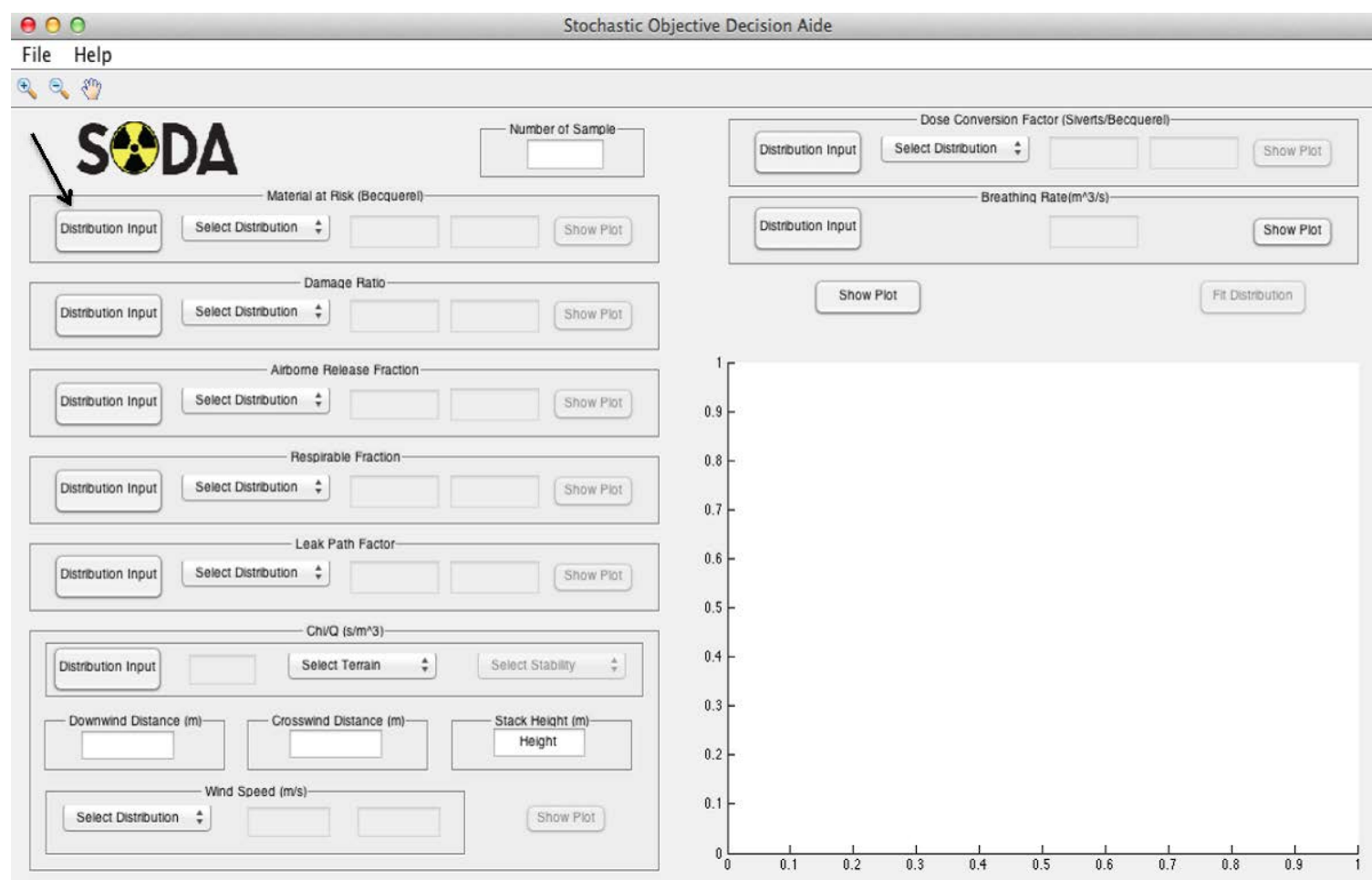

To switch from distribution to single input, click on

"Distribution Input".

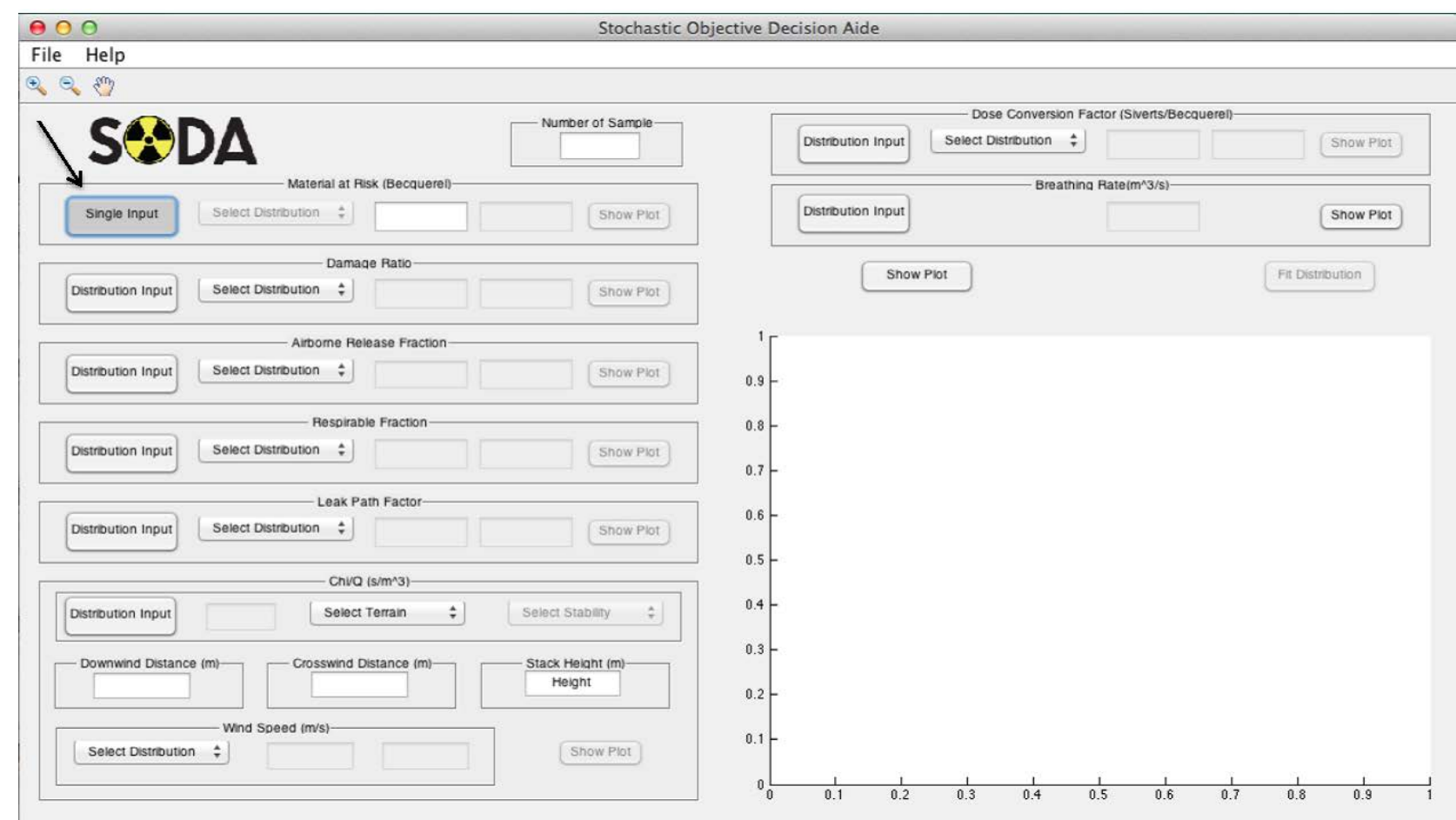

For a single input, enter the number in Becquerel's. 


\section{A.6.3 Entering Values for Damage Ratio Parameters}

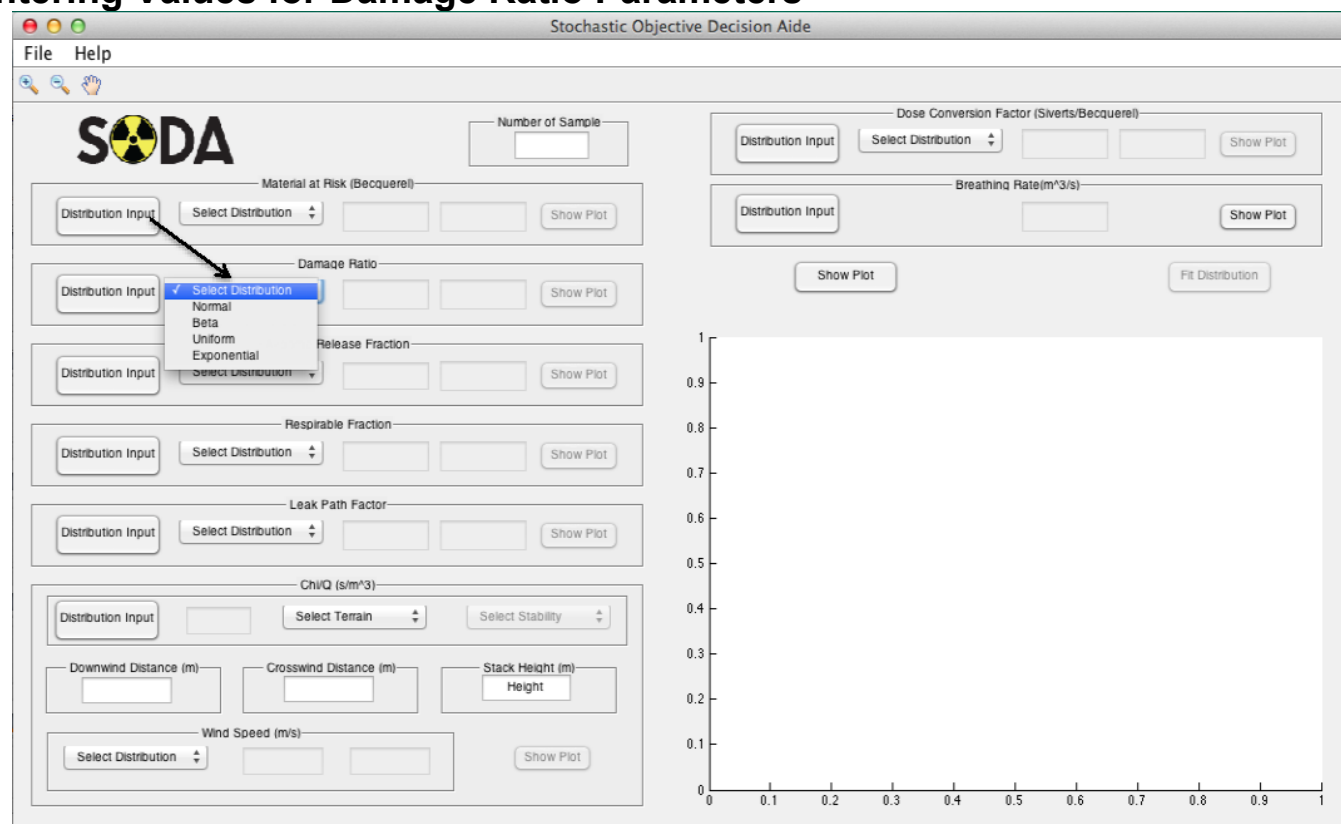

In Damage Ratio, for a distribution input, choose the distribution type (Normal, Beta, Uniform, Exponential)

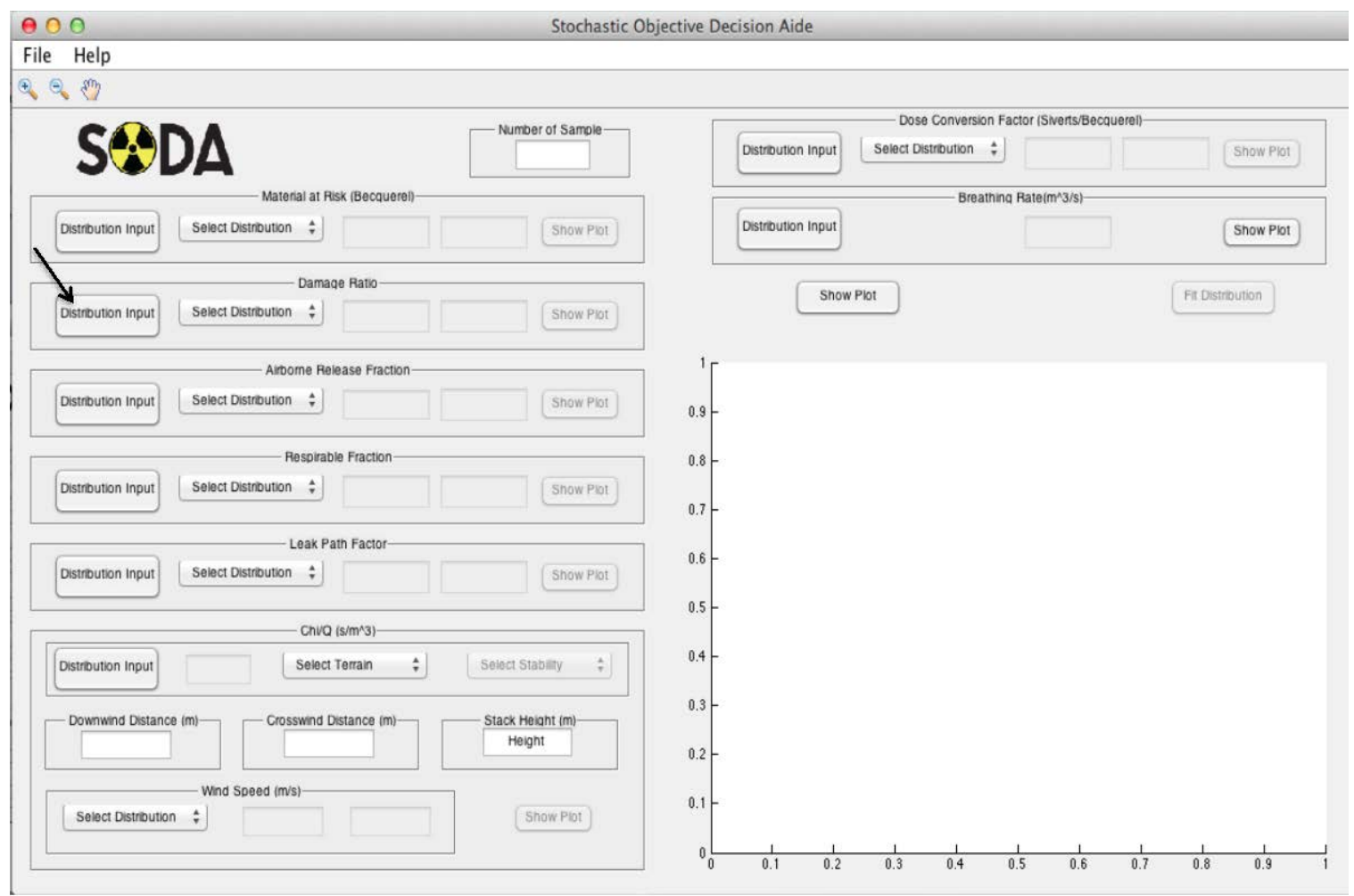

To switch from distribution to single input, click on "Distribution Input" 


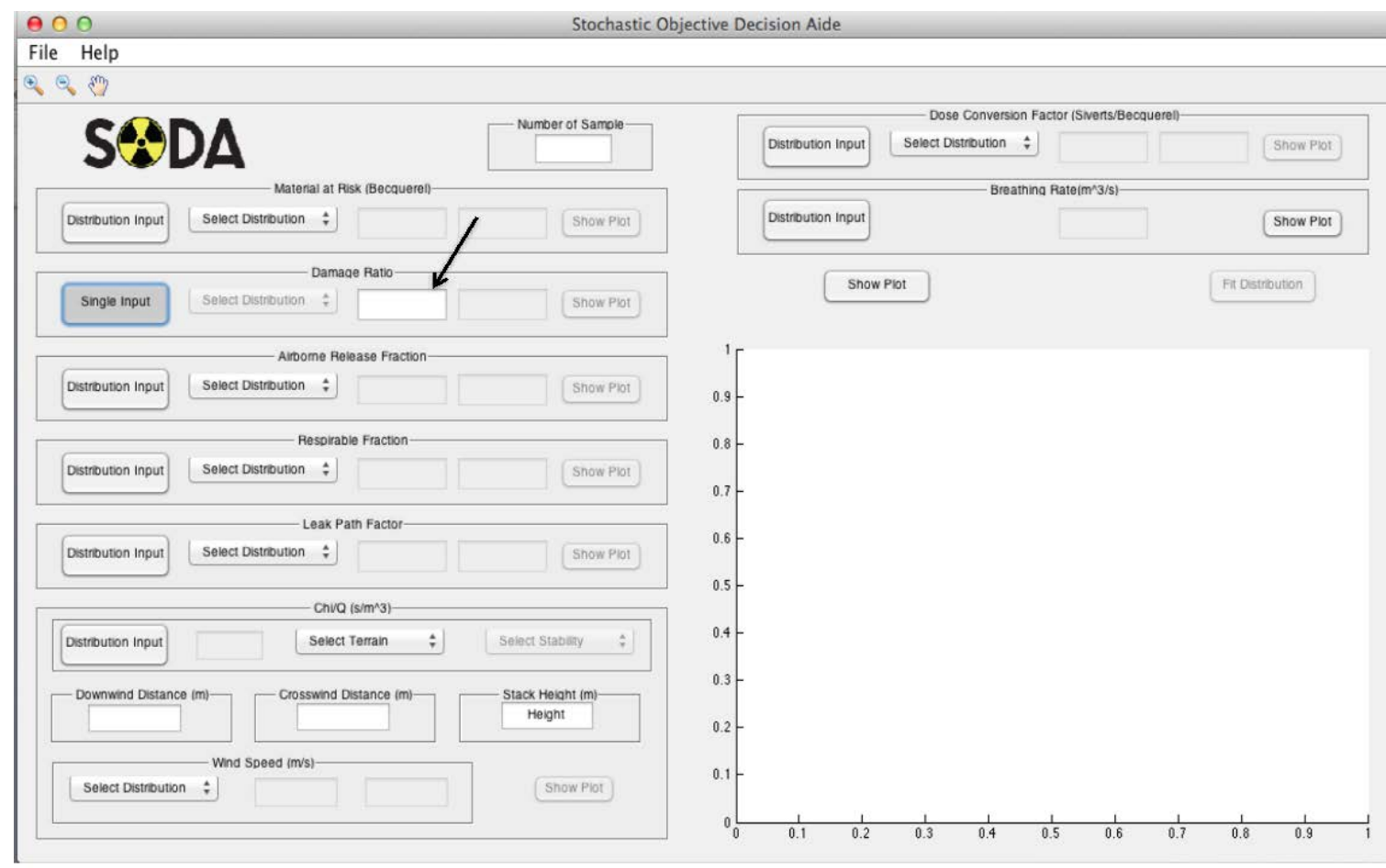

For a single input, enter the number in Becquerel's

\section{A.6.4. Entering Values for ARF Parameters}

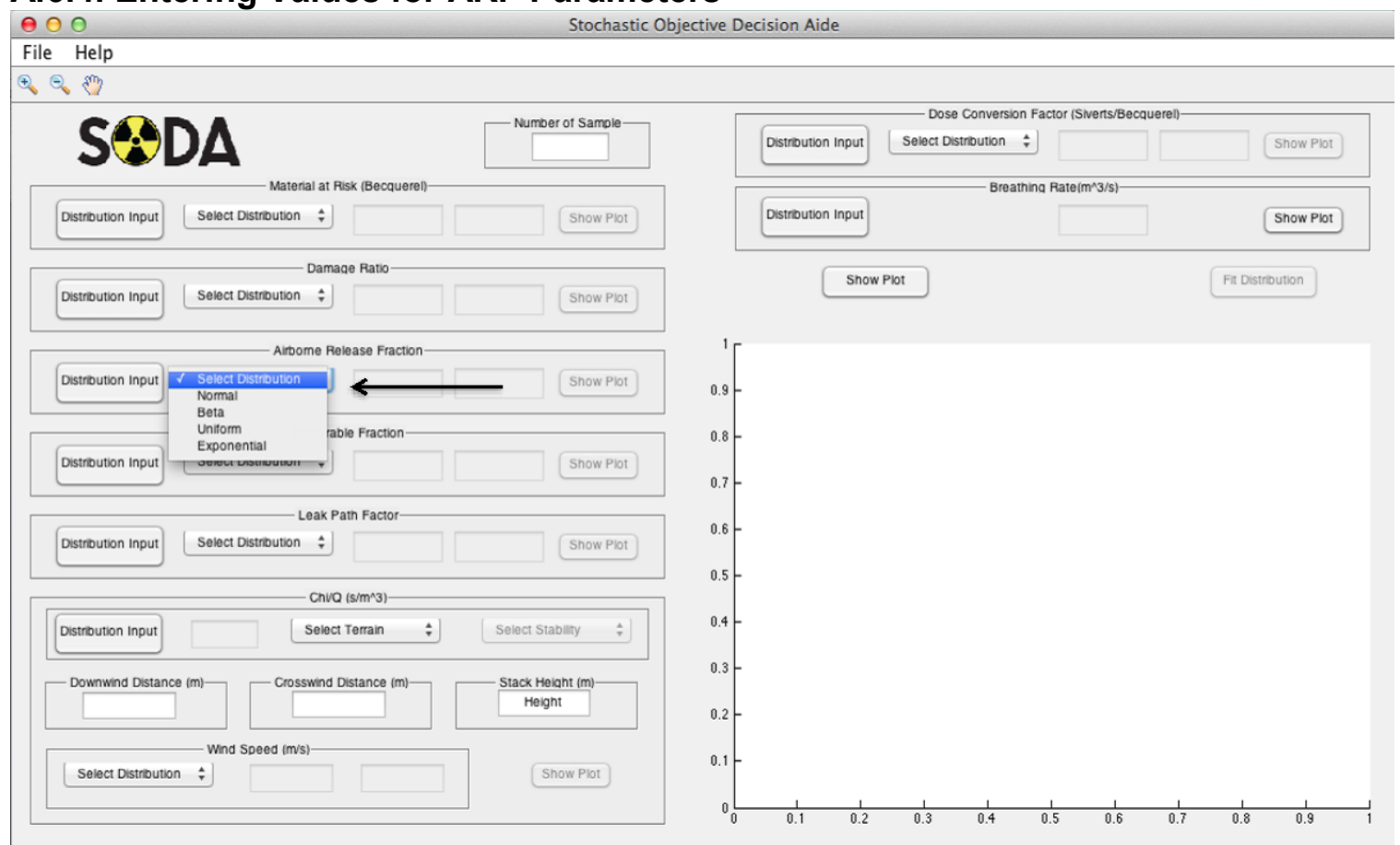

In Airborne Release Fraction, for a distribution input, choose the distribution type (Normal, Beta, Uniform, Exponential) 


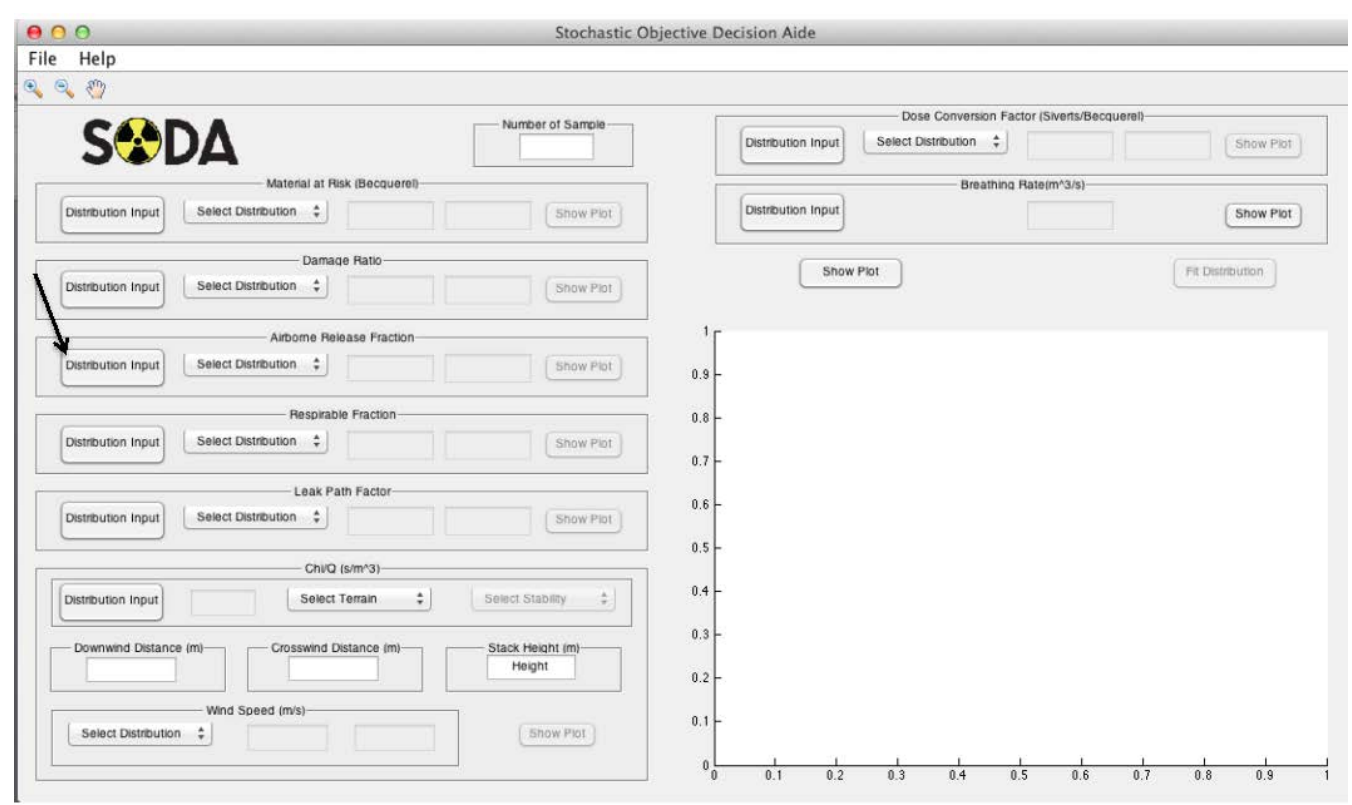

To switch from distribution to single input, click on "Distribution Input".

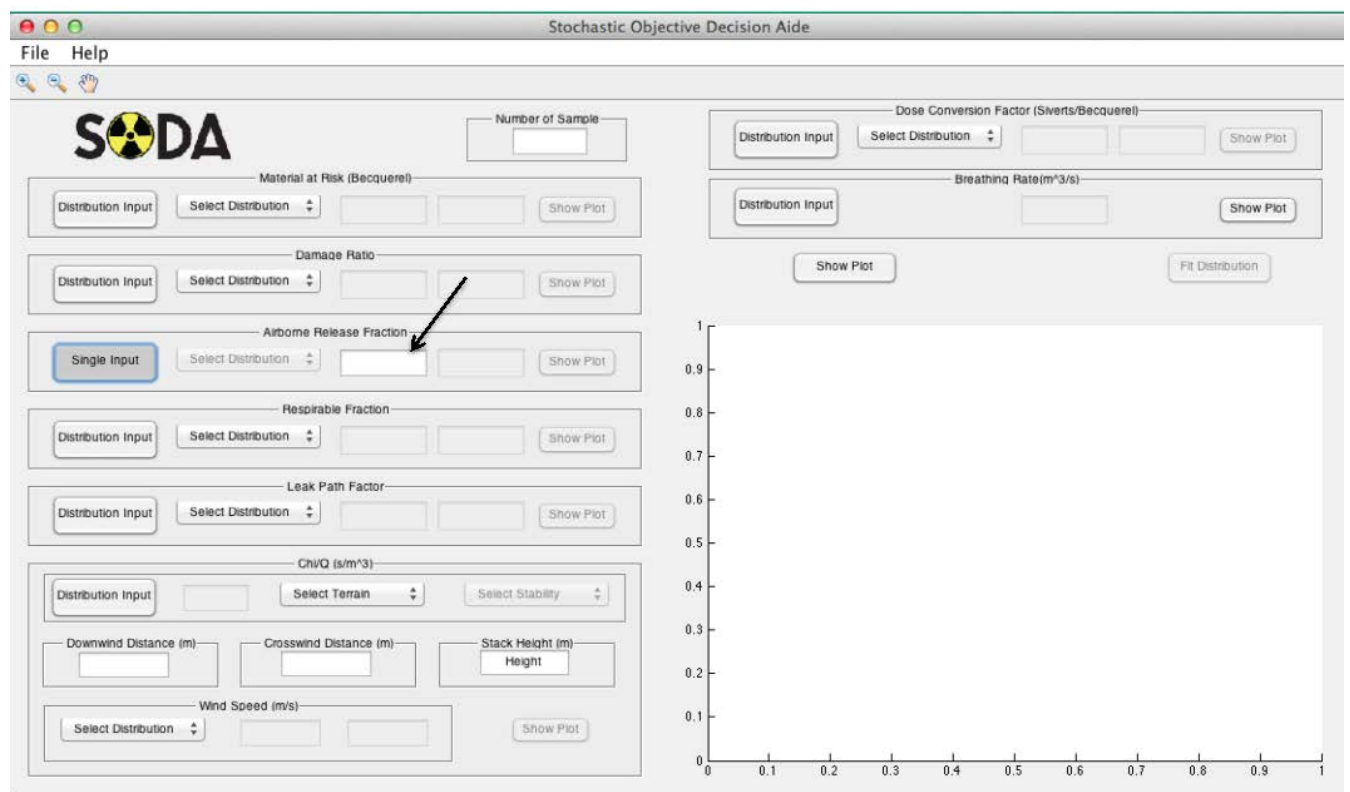

For a single input, enter the number in Becquerel's 


\section{A.6.5. Entering Values for Respirable Fraction Parameters}

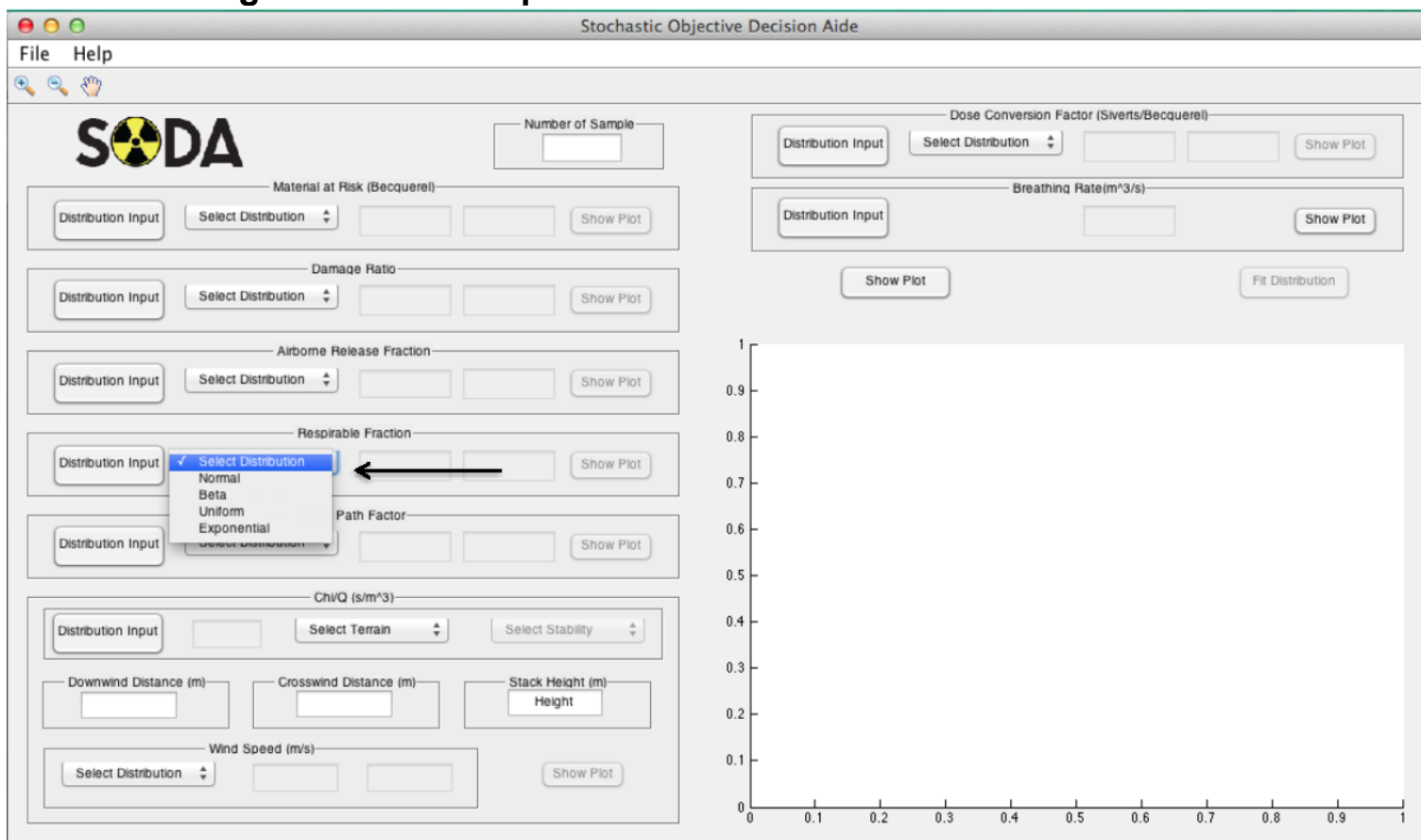

In Respirable Fraction, for a distribution input, choose the distribution type (Normal, Beta, Uniform, Exponential)

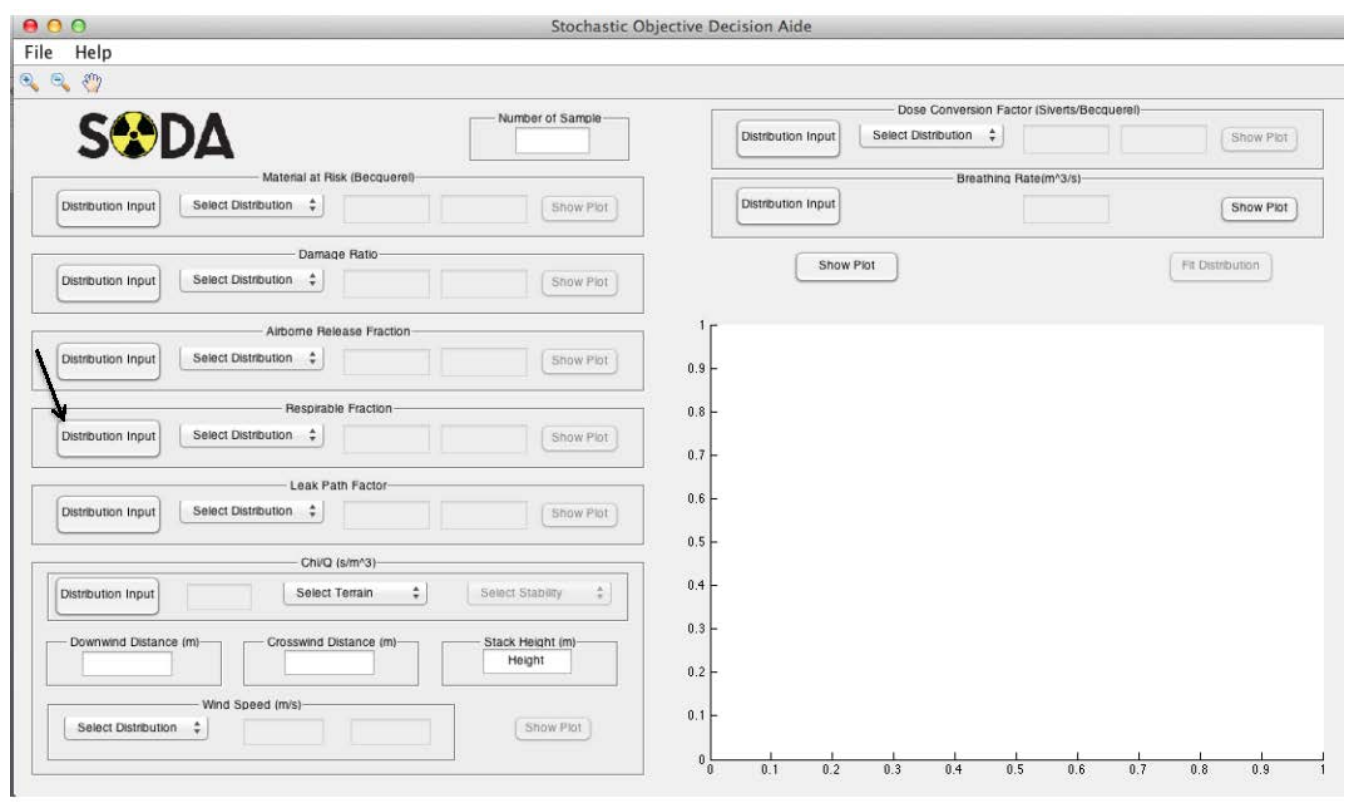

To switch from distribution to single input, click on "Distribution Input" 


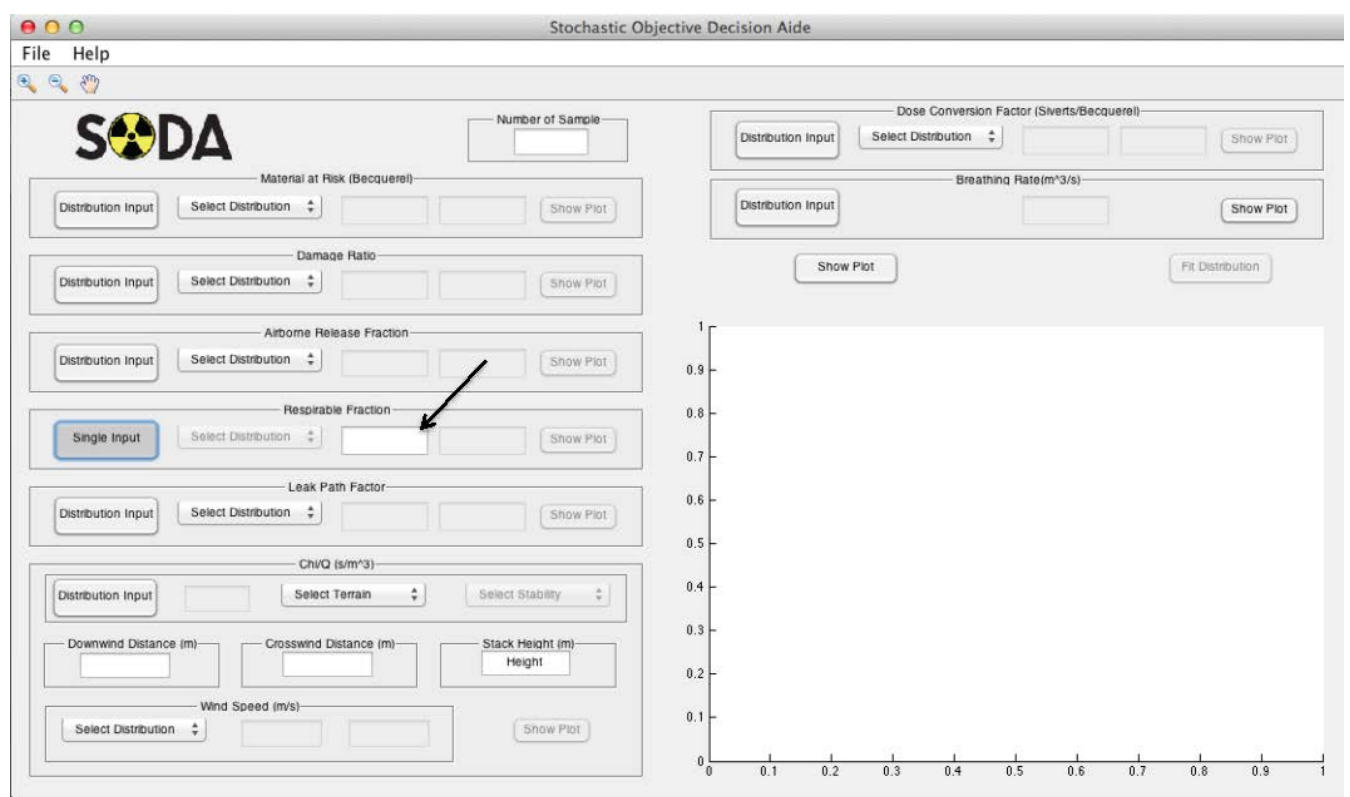

For a single input, enter the number in Becquerel's

\section{A.6.6. Entering Values for LPF Parameters}

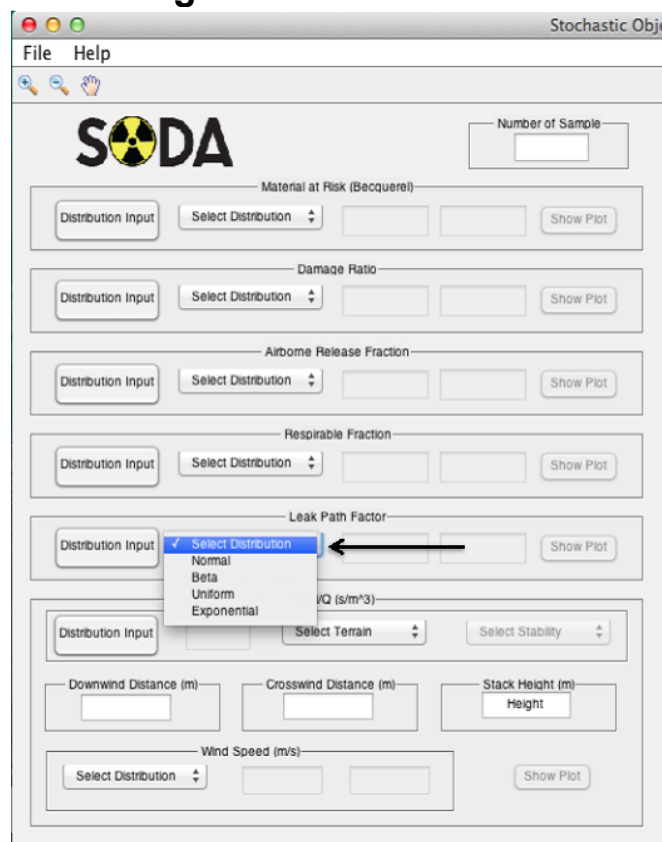

Decision Aide

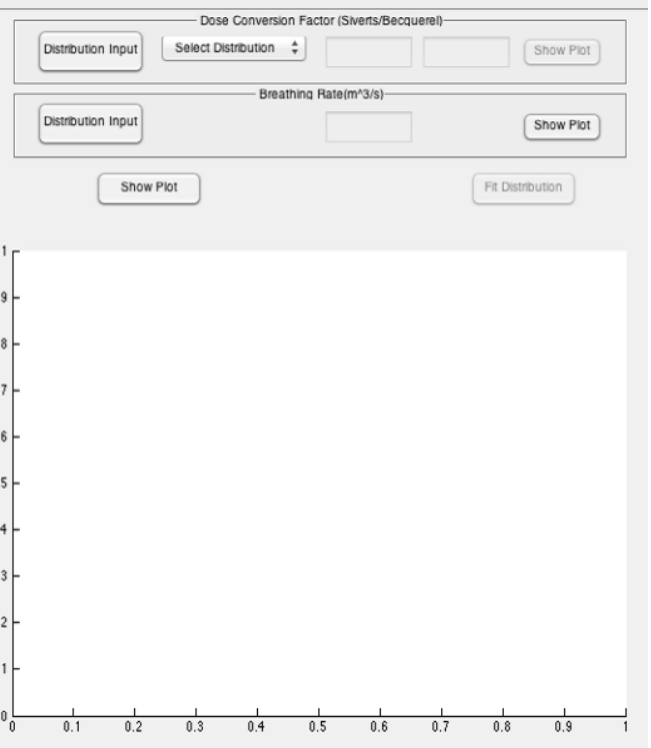

In Leak Path Factor, for a distribution input, choose the distribution type

(Normal, Beta, Uniform, Exponential) 


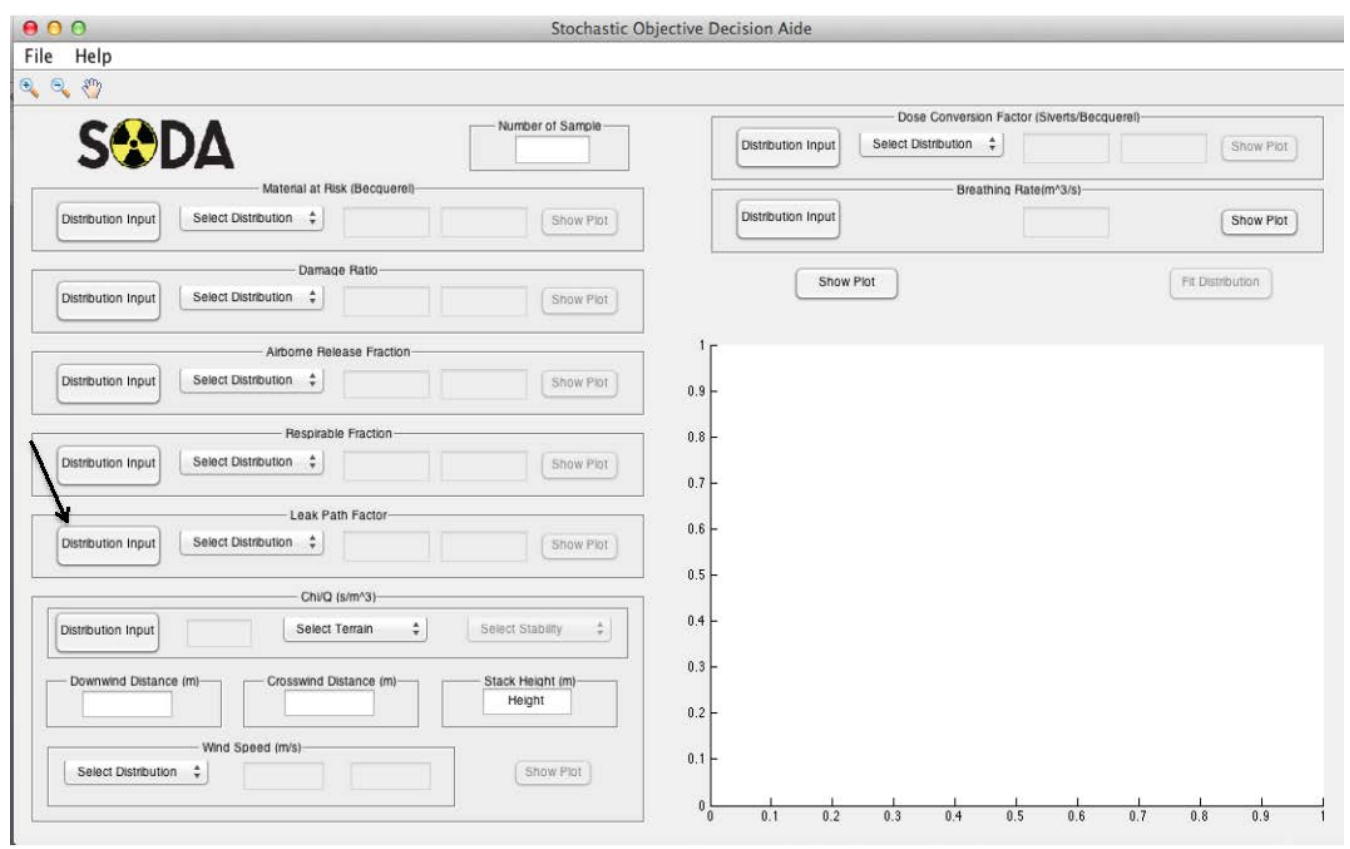

To switch from distribution to single input, click on "Distribution Input"

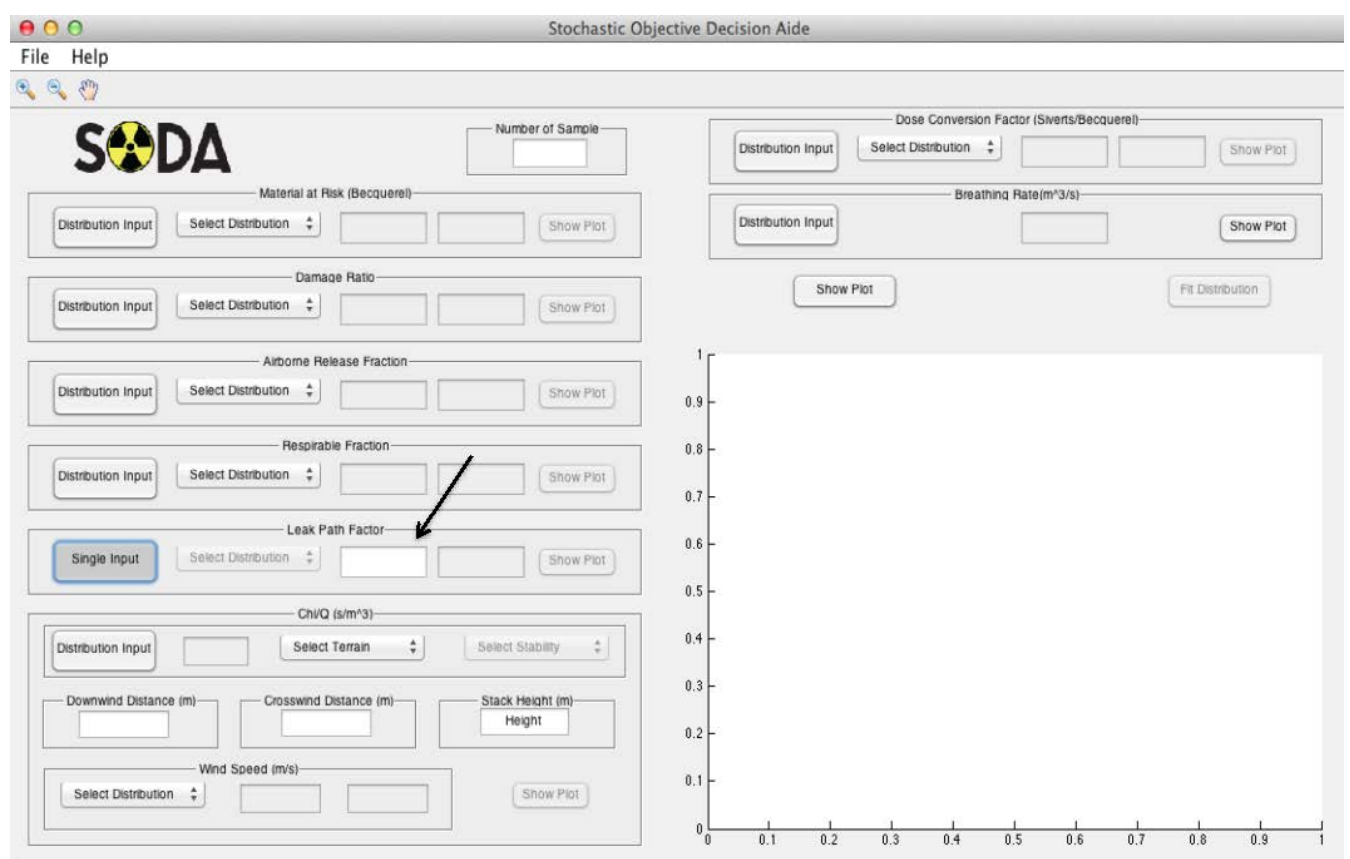

For a single input, enter the number in Becquerel's 


\section{A.6.7. Select Terrain for $\mathrm{Chi} / \mathrm{Q}$}

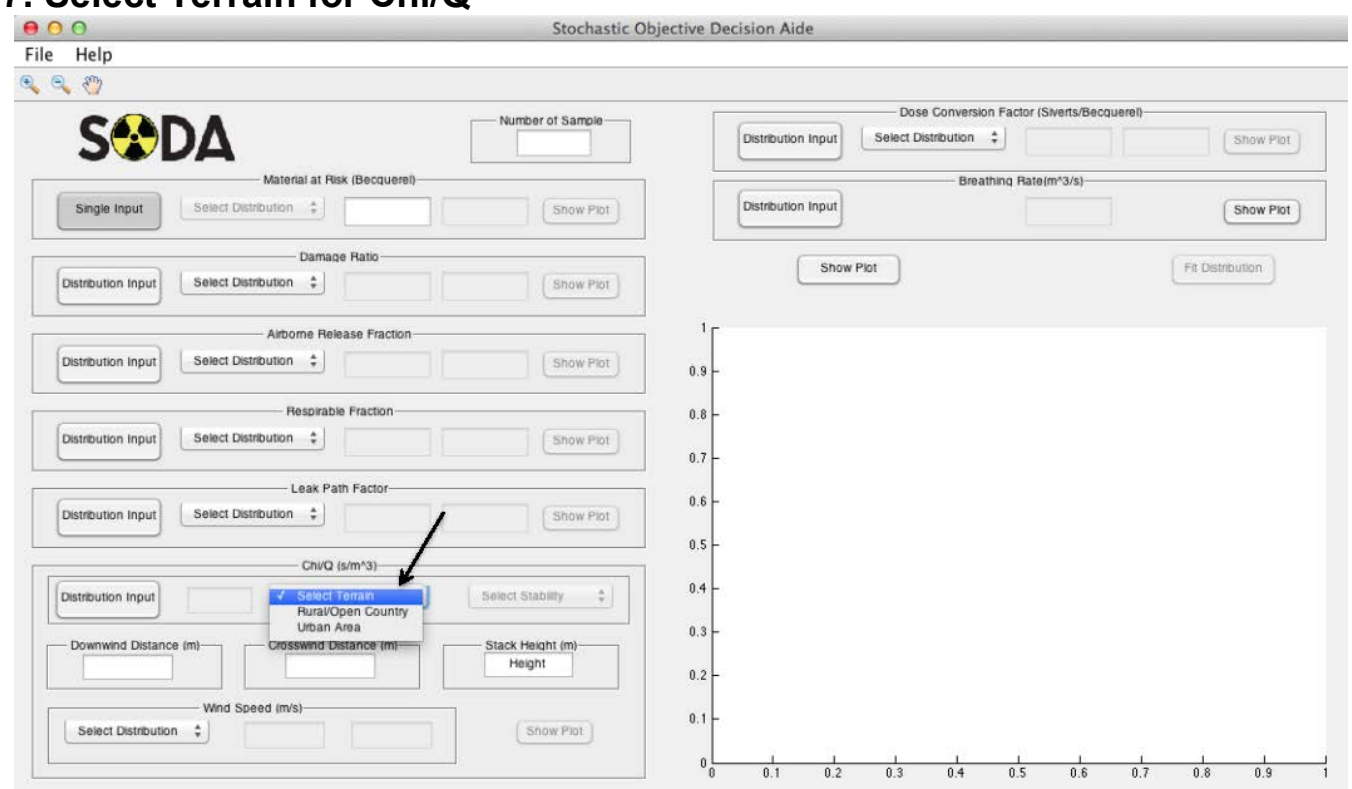

For Chi/Q distribution input, choose the terrain (Open country or Urban area)

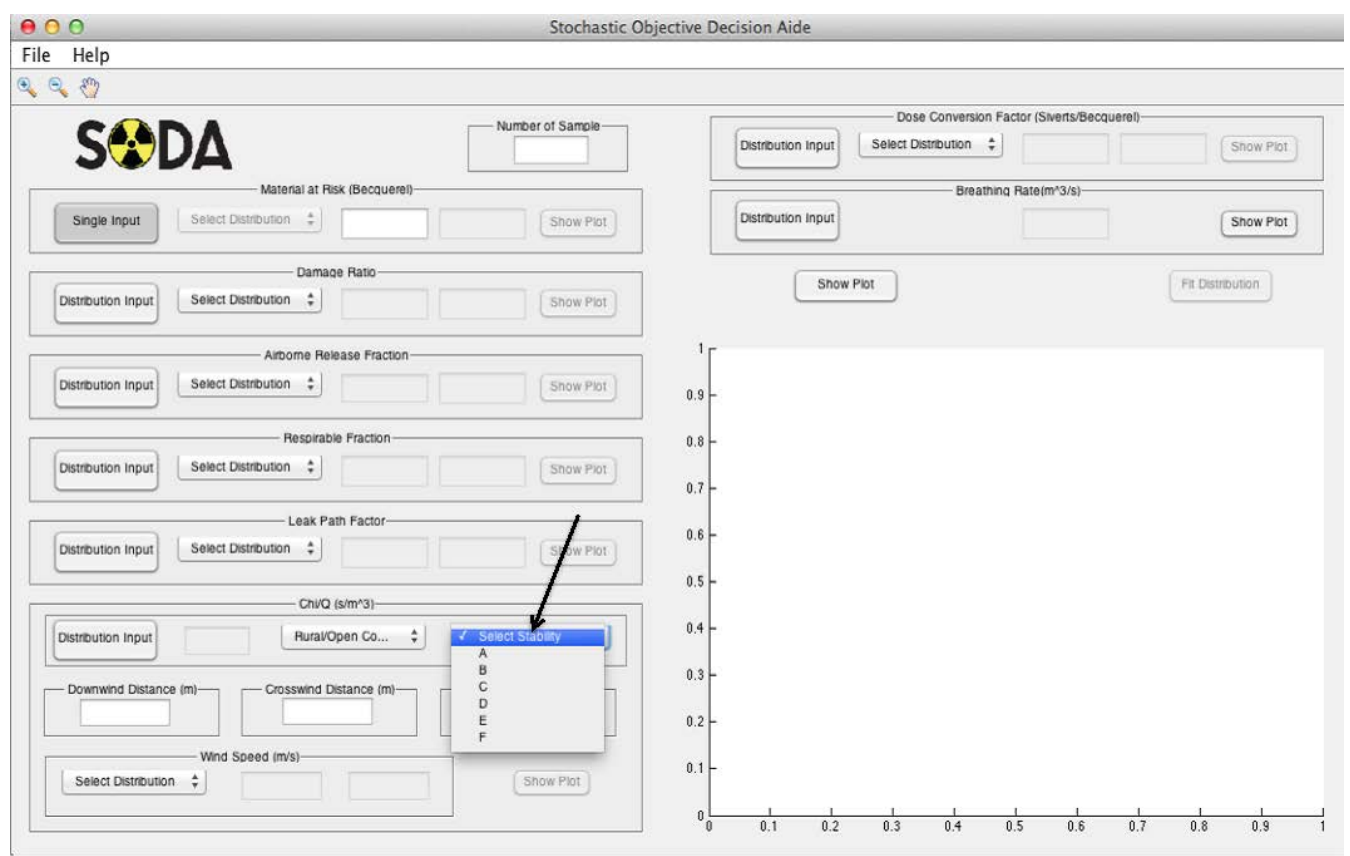

For Chi/Q distribution input, if you choose Open country, then choose the stability $(A, B, C, D, E, F)$ 


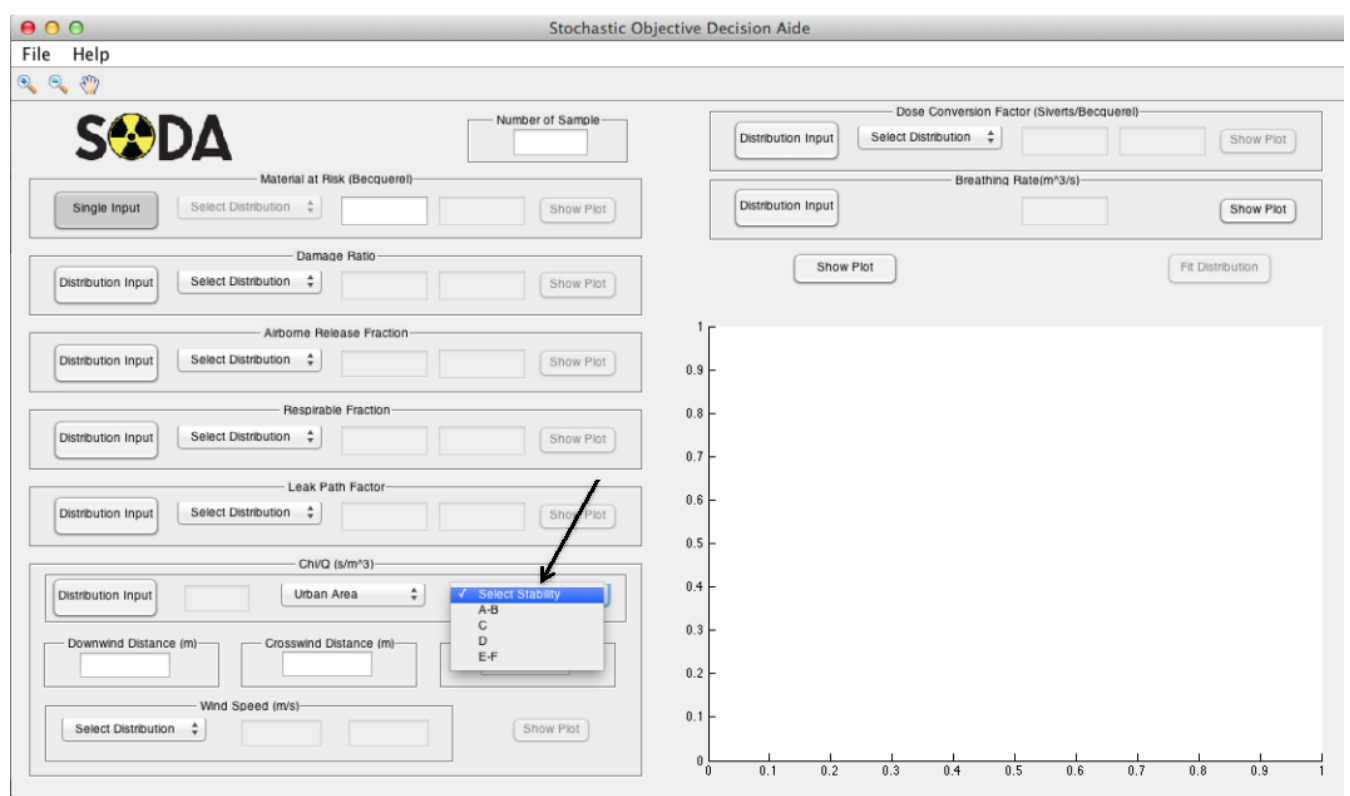

For Chi/Q distribution input, if you choose Urban Area, then choose the stability $(A-B, C, D, E-F)$

\section{A.6.8. Entering Values for Downwind Distance, Crosswind Distance, and Stack Height}
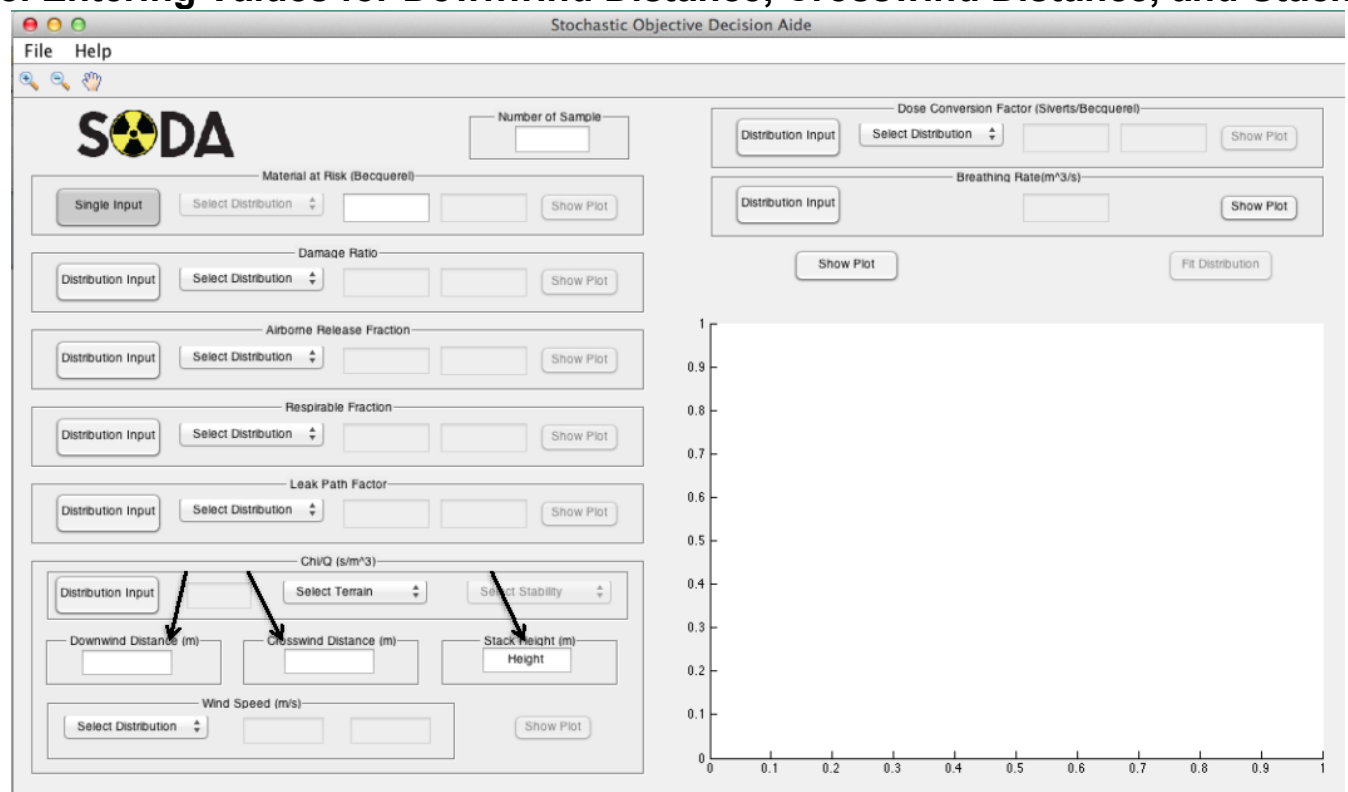

After that, enter the numbers of Downwind Distance, Crosswind Distance, and Stack Height. (All in meter) 


\section{A.6.9. Select Distribution for Wind Speed}

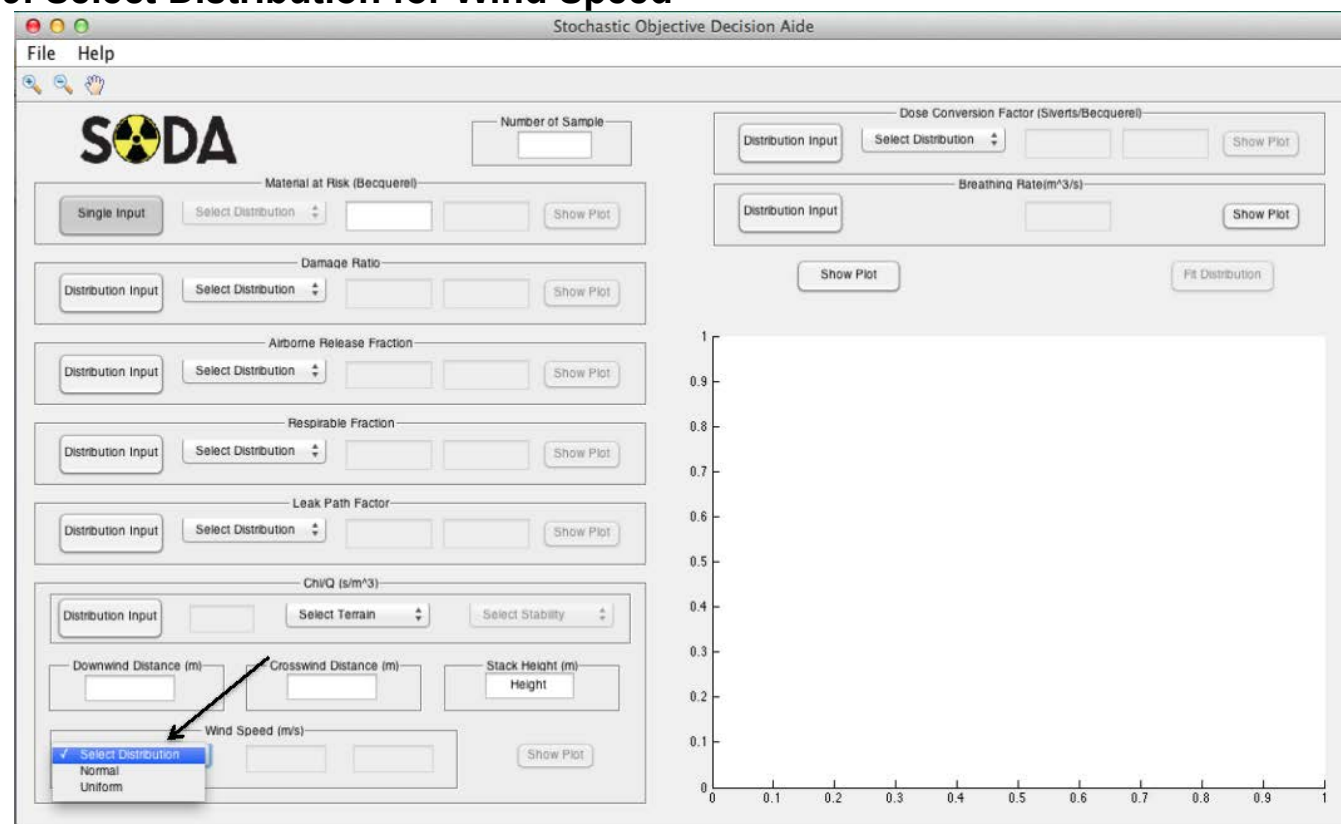

For Wind speed, select the distribution

\section{A.6.10. Entering Values for Dose Conversion Factor Parameters}
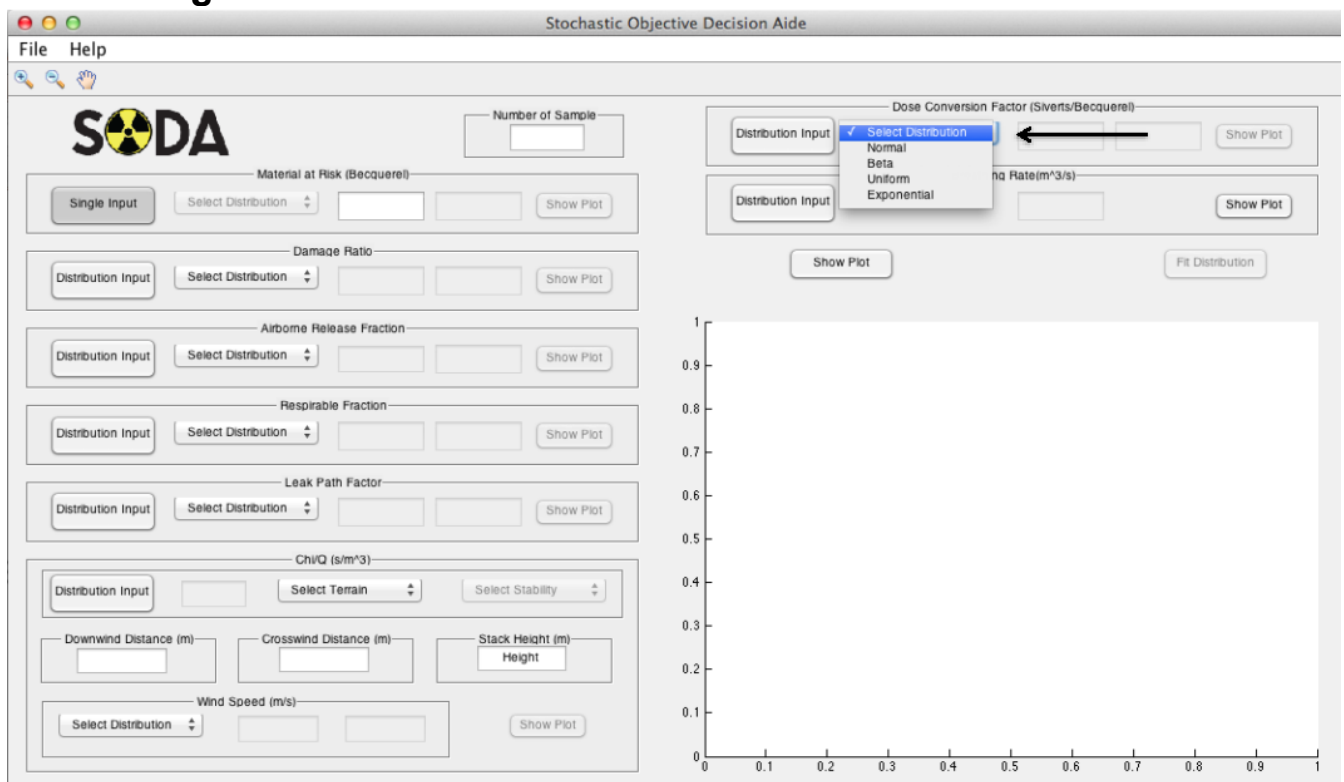

In Dose Conversion Factor, for a distribution input, choose the distribution type (Normal, Beta, Uniform, Exponential) 


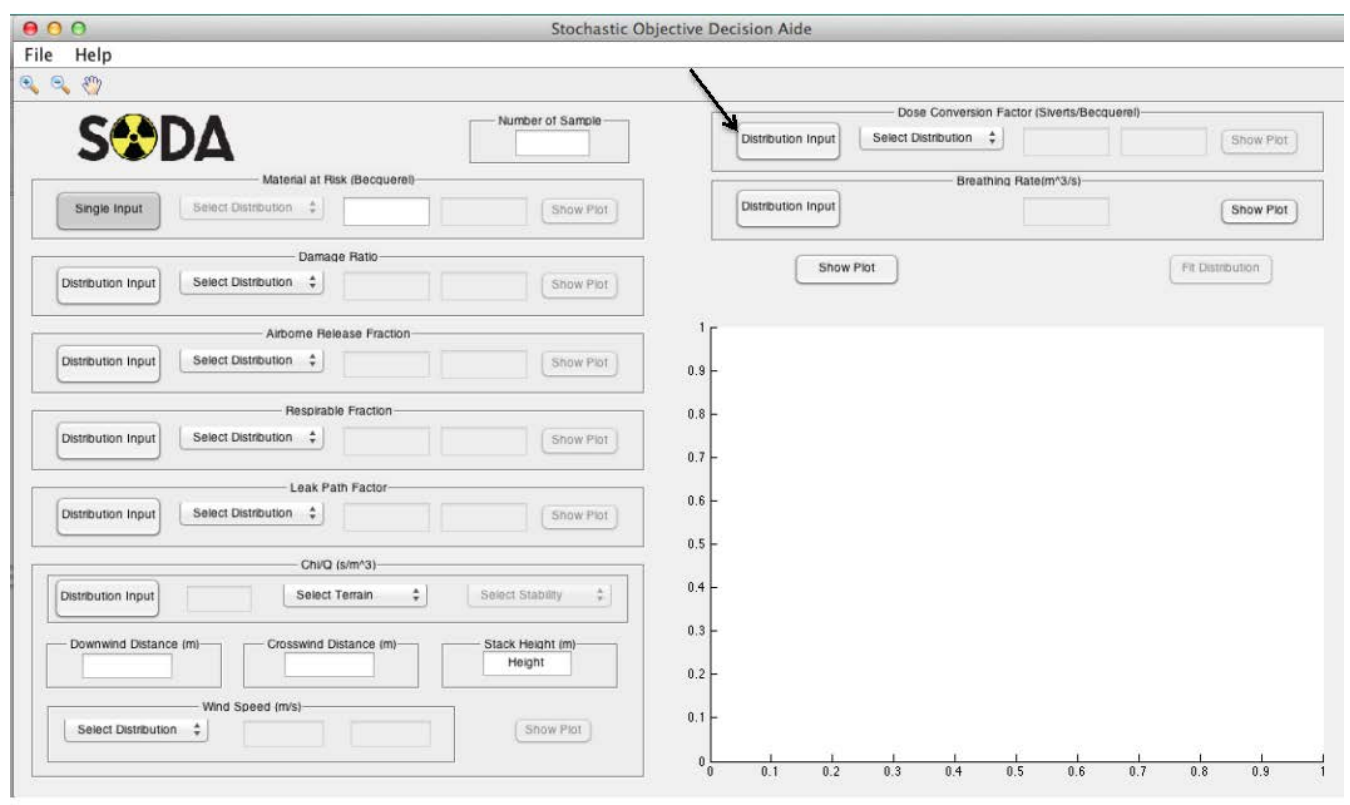

To switch from distribution to single input, click on "Distribution Input"

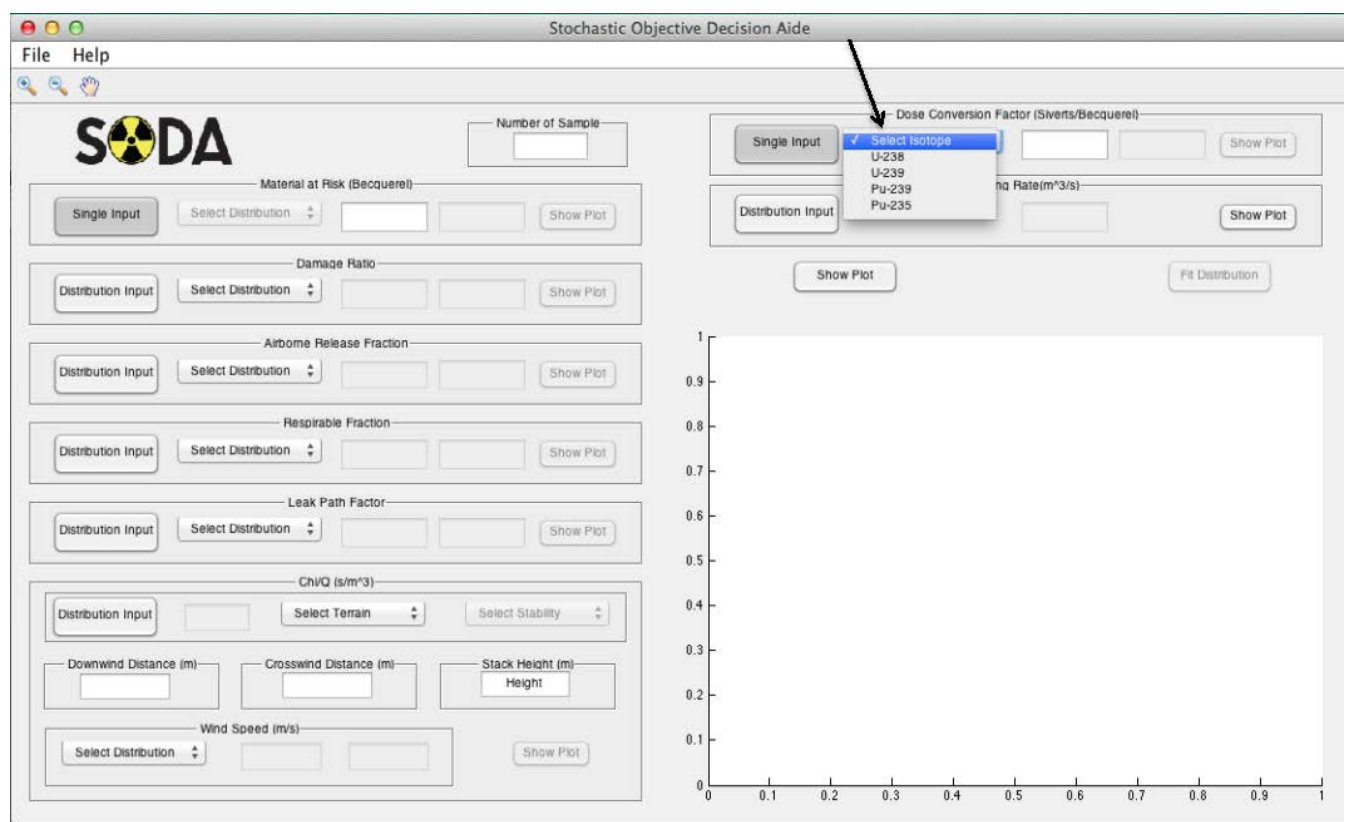

For a single input, select the isotope 


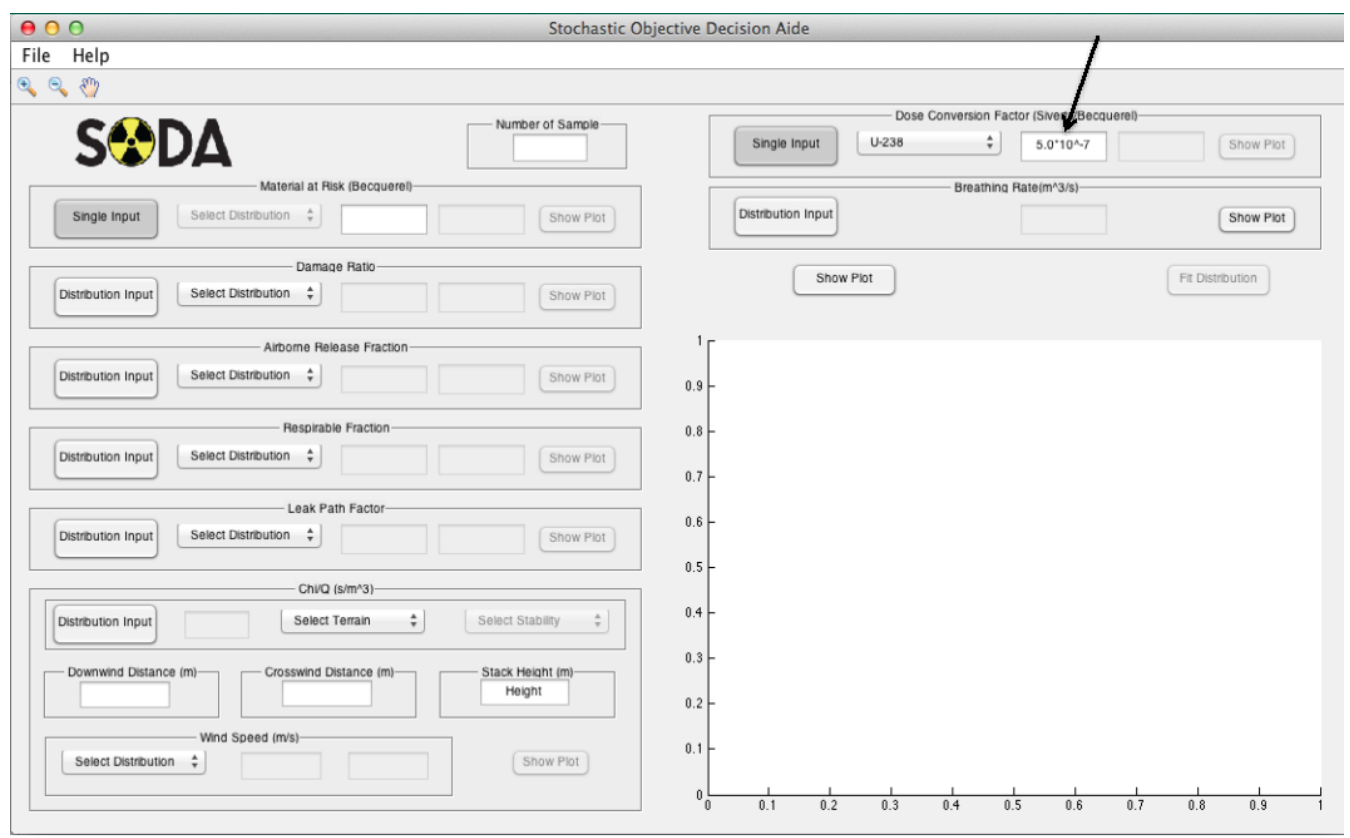

For example, select U-238 and its Dose Conversion Factor will show

\section{A.6.11. Entering Values for Breathing Rate Parameters}

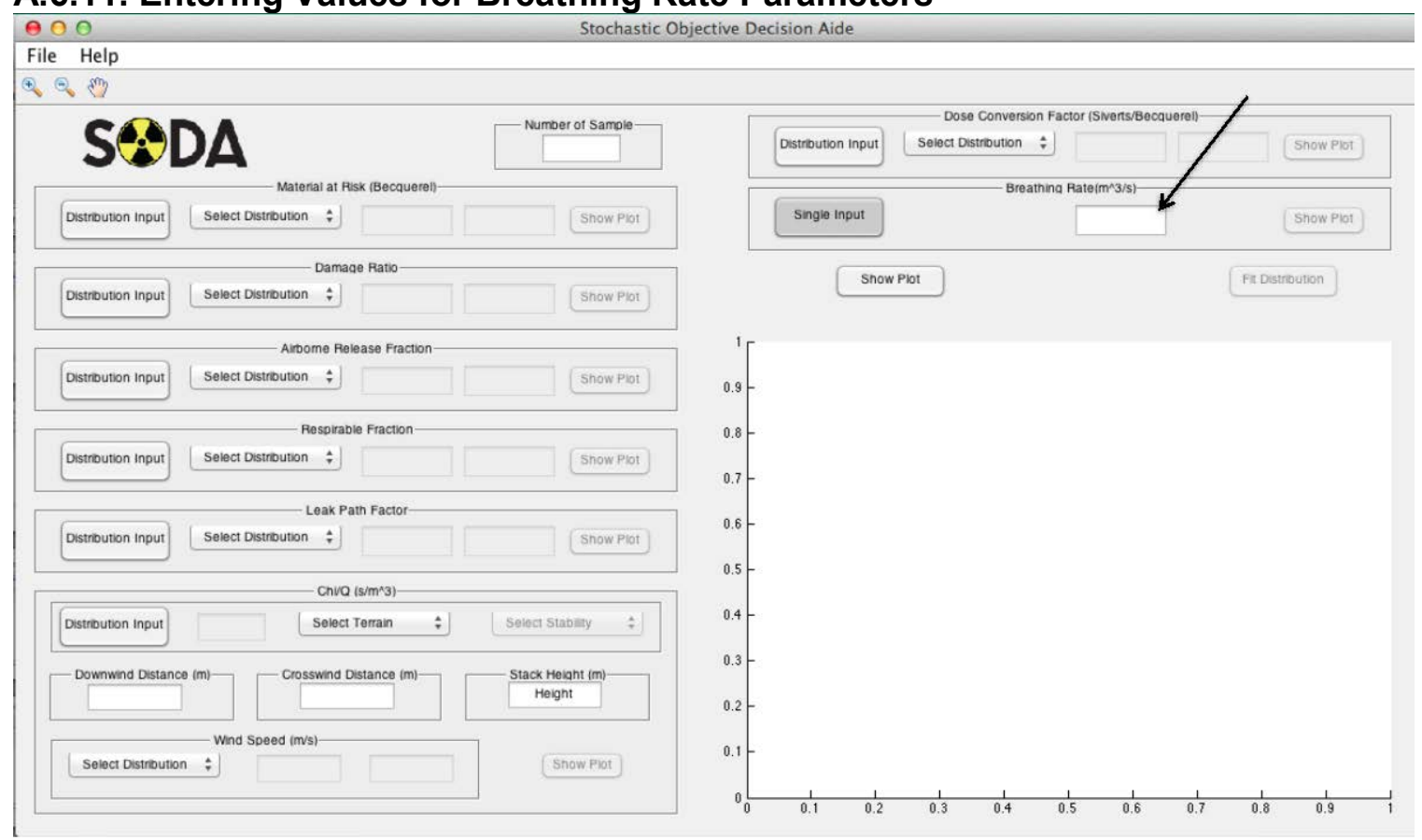

For Breathing rate, enter the number in $\left(\mathrm{m}^{3} / \mathrm{s}\right)$ 
INL/EXT-15-36669

Rev. 0 September 2015

Page 38 of 129

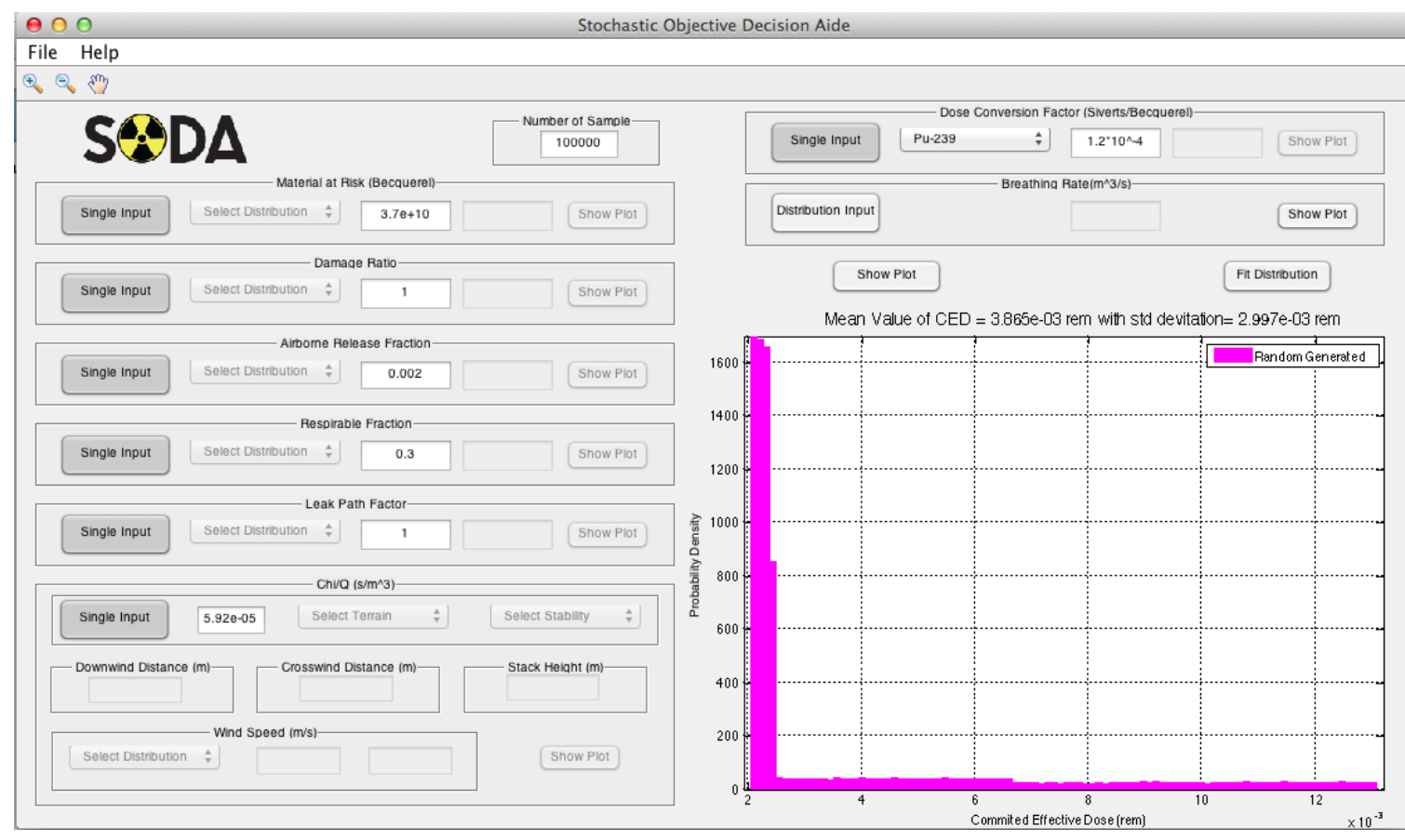

th assumptions of 24 hours

Breathing rate 


\section{APPENDIX B: \\ Installation Instructions}

SODA installation instruction for both Mac and Windows PCs are provided below.

\section{Mac Installation}

1. Download the program, and the download file will be in the downloads folder. Click to open the installation program.

2. The install application will take a few minutes to load. Please wait then put in your name and password.

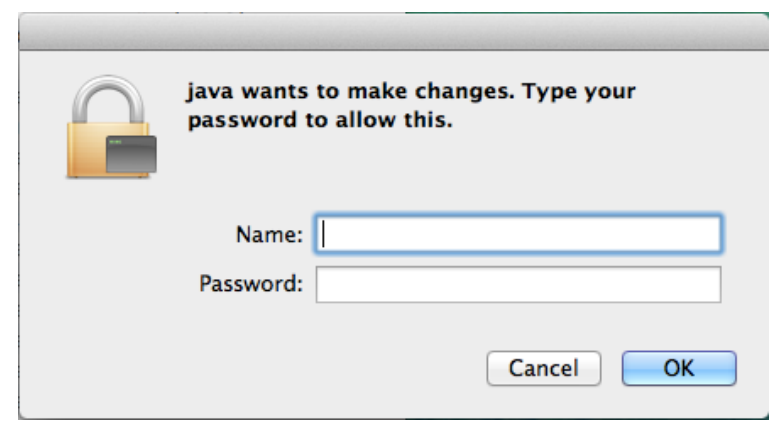

3. When you see the NSRD installer window, click "next" to continue Installation.

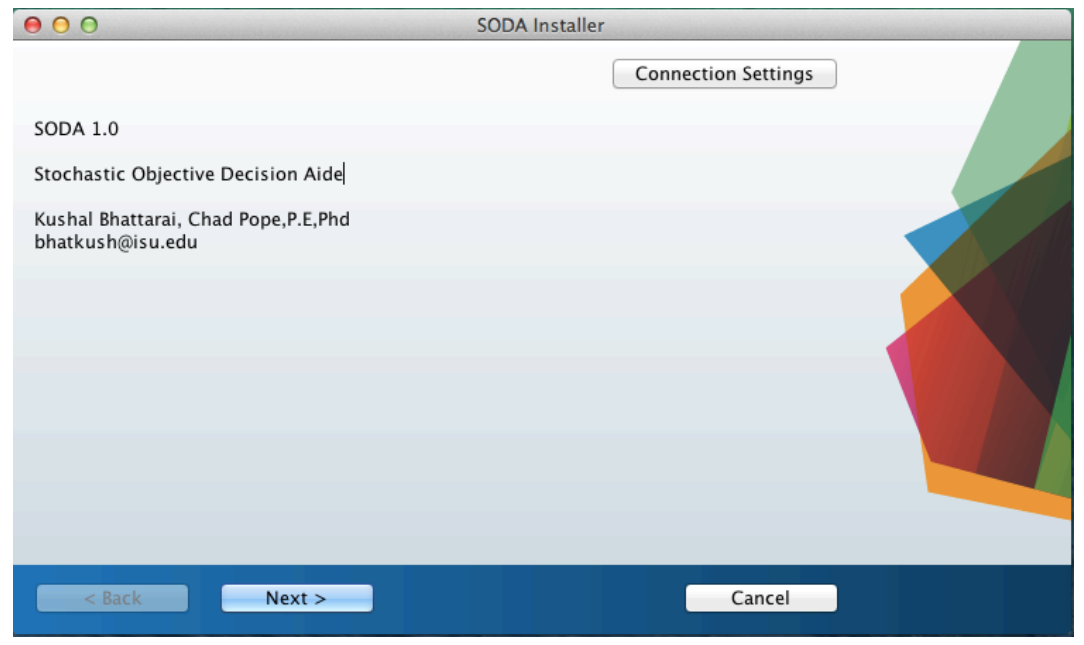


4. Please wait for the Contacting Math Works to prepare the installation; then you will see the "Installation Options" window.

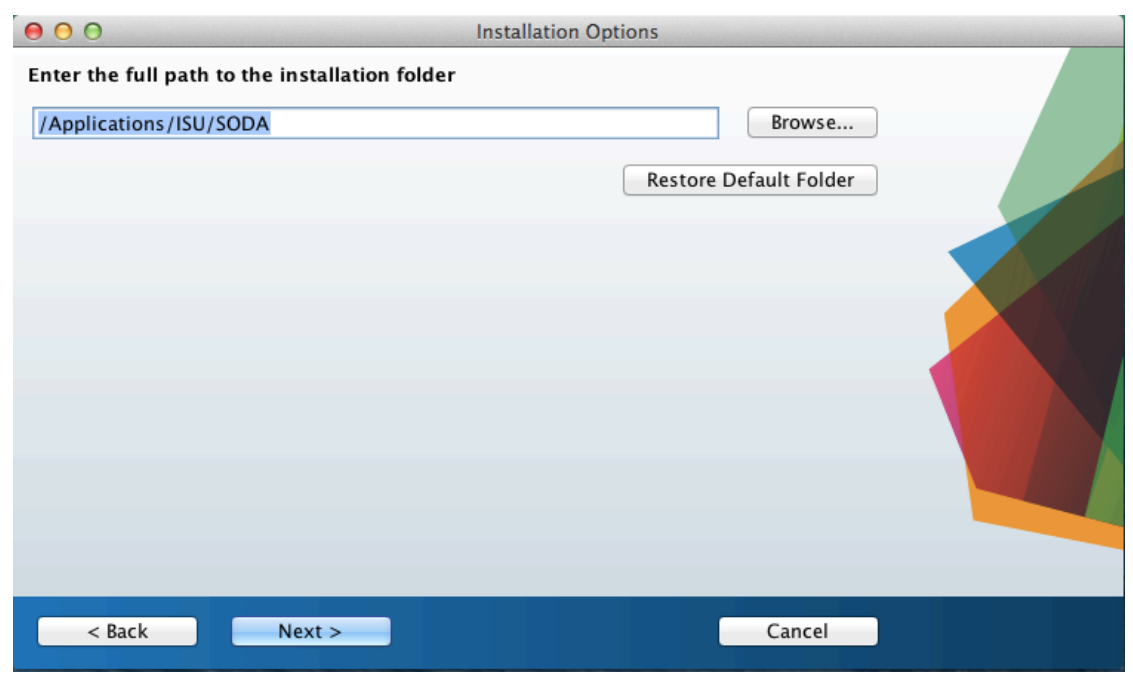

5. Click on the browse button to set a location for the program to be installed.

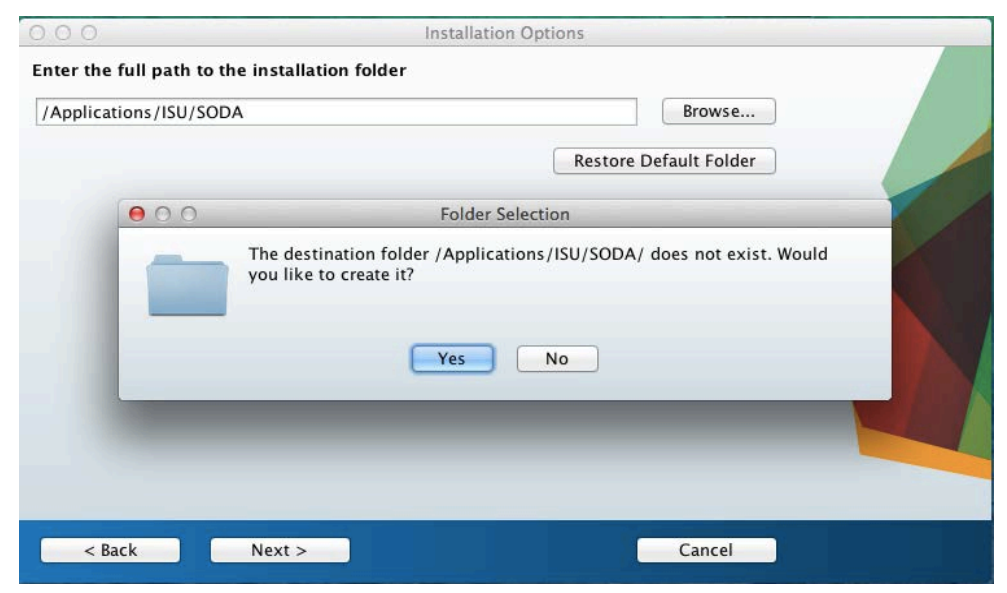

6. Click "next" to continue installation. 
7. "Required software" window will show up; click on the browse button to set a location for the program to be Installed.

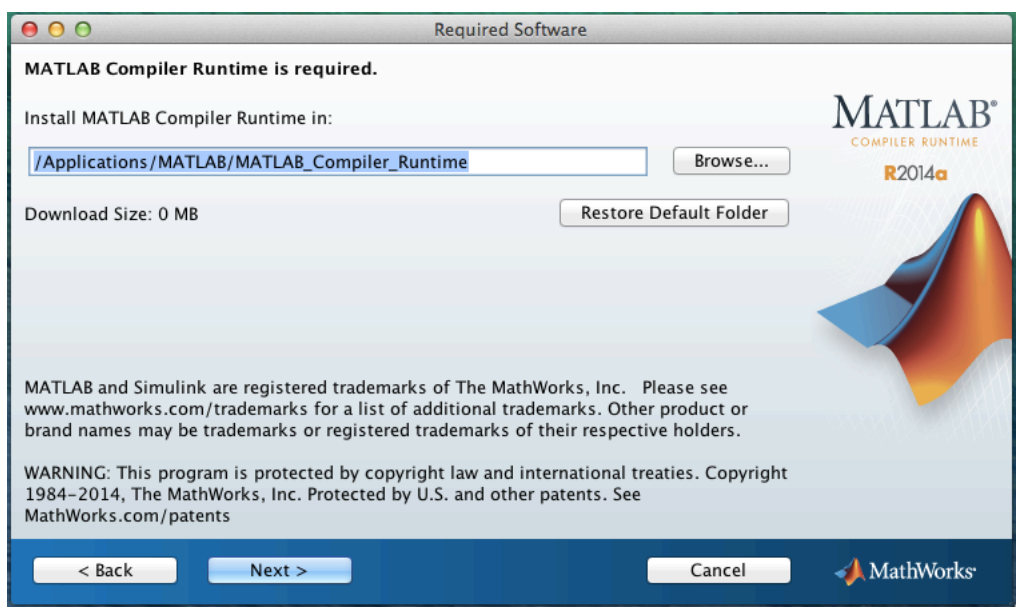

8. Click next to continue installation.

9. When you see the "License agreement" window, click the Yes button to accept the License Agreement. Note: If you check No the program will cancel.

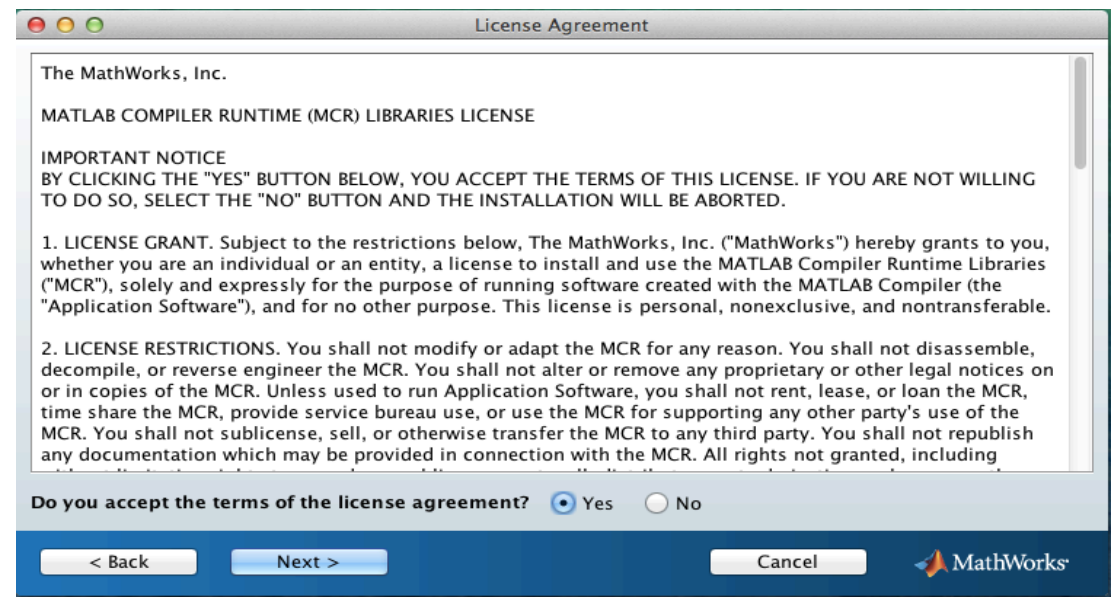

10. Click Next to continue installation. 
11. Confirmation window will show up; click "install" to start downloading the program.

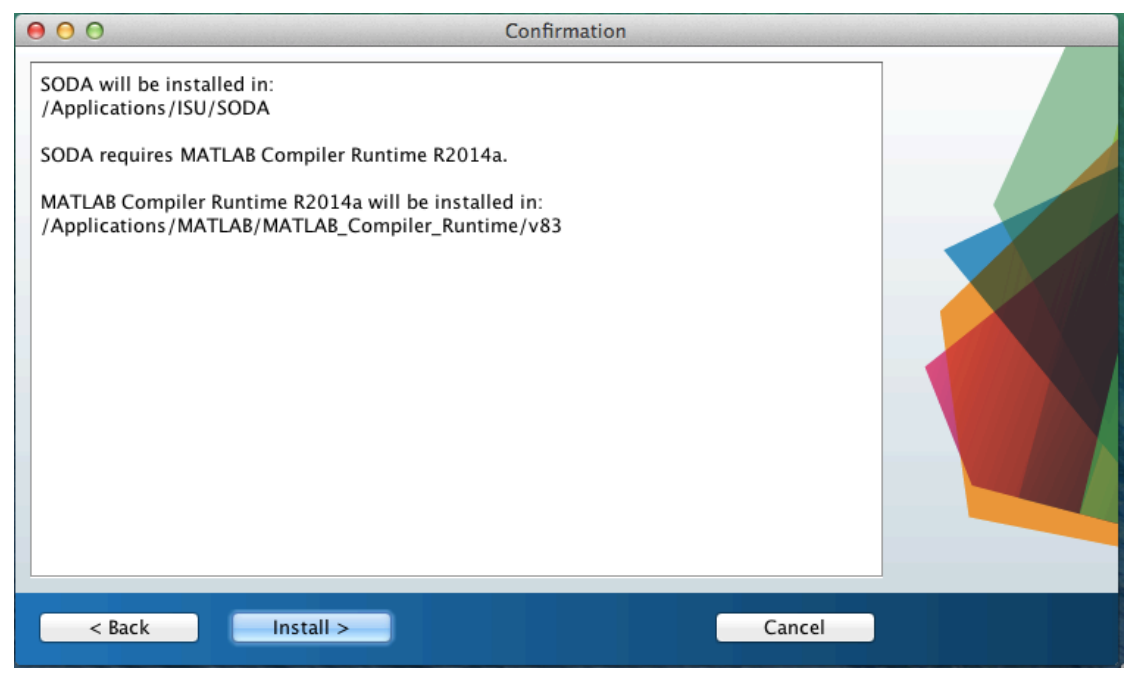

12. The download will keep track of the percentage downloaded $0 \%-100 \%$. If you need to pause the program for any reason, press the pause button. Once the program reaches $100 \%$, the "Installation complete" window will show.

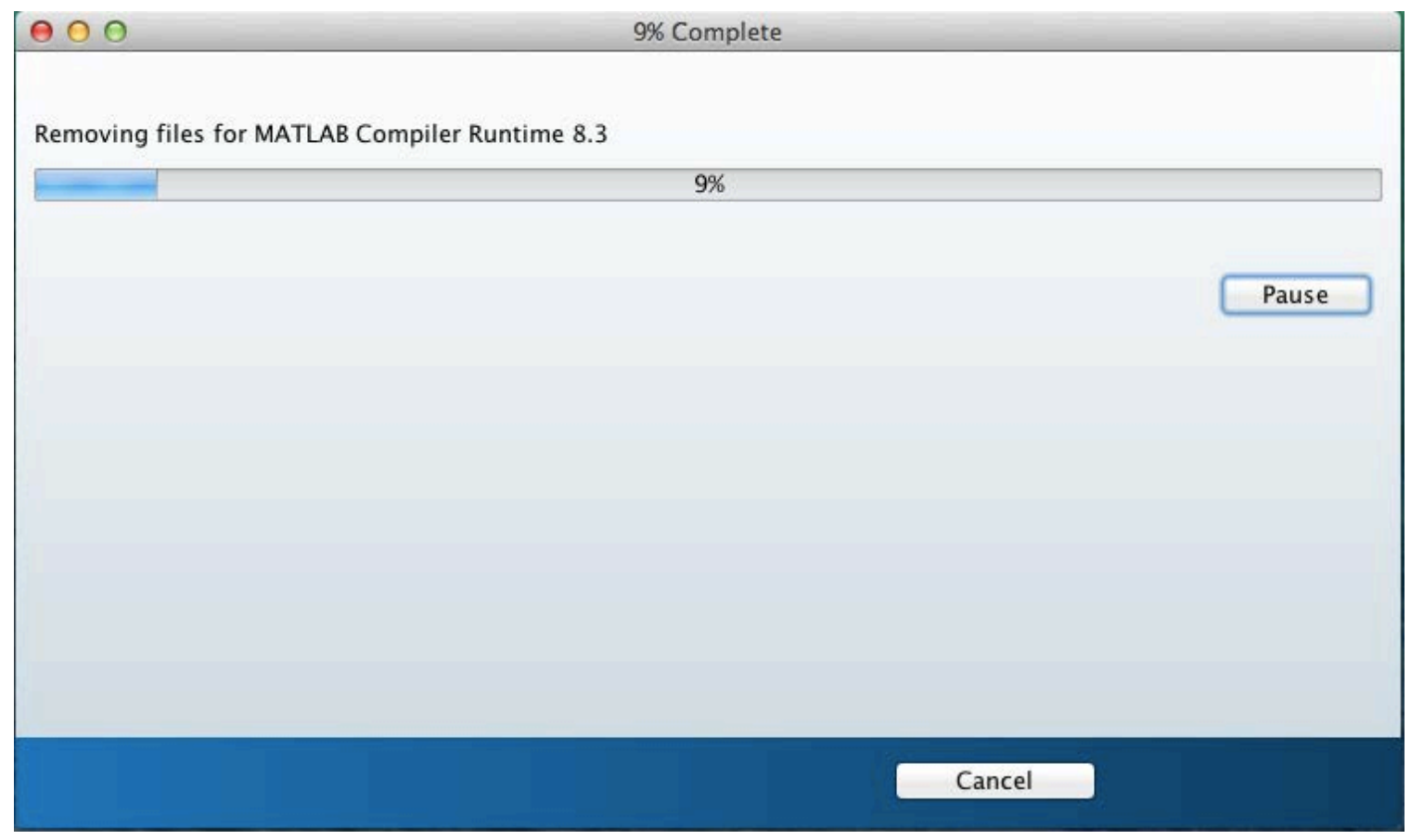

13. Click finish; the program can be found in the location that you chose. 


\section{Windows Installation}

1. Download the program; the download file will be at the bottom of your Internet browser. Click to open the installation program.

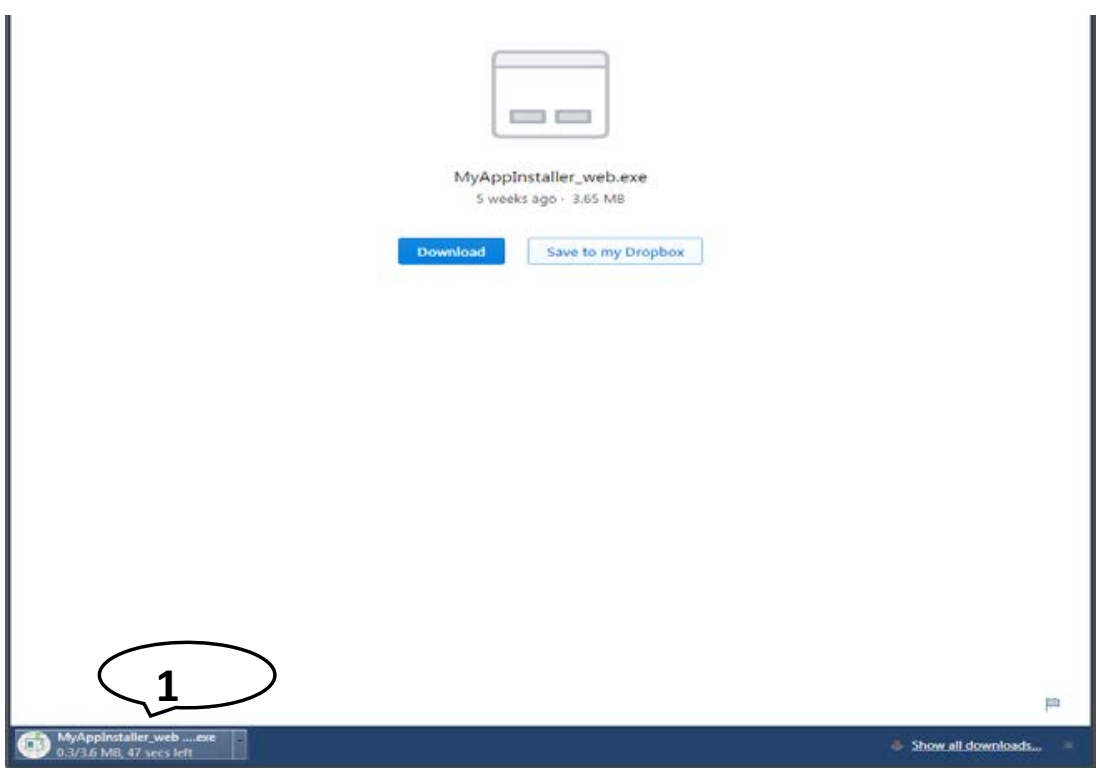

2. The install application will take a few minutes to load. Please just let it run until you see the SODA Installer window.

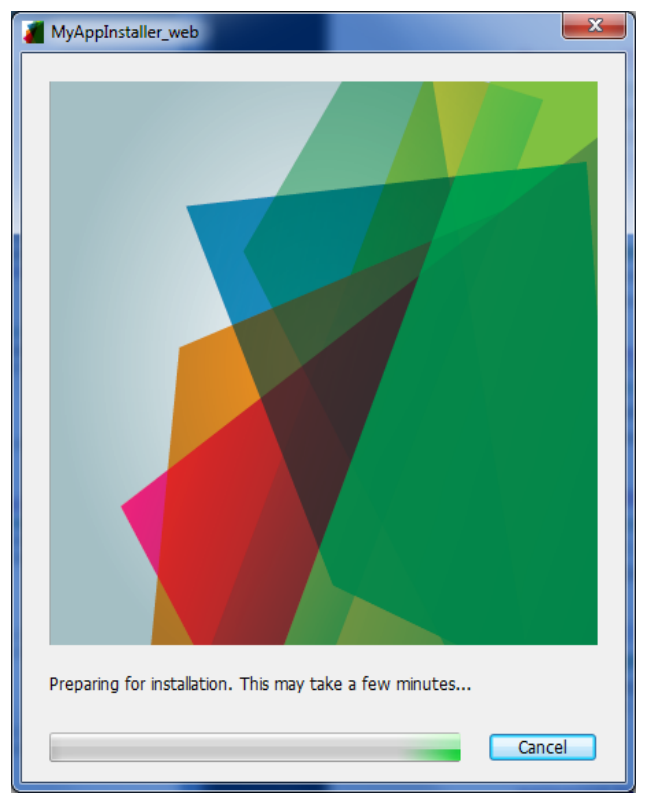


3. Click next button to continue Installation.

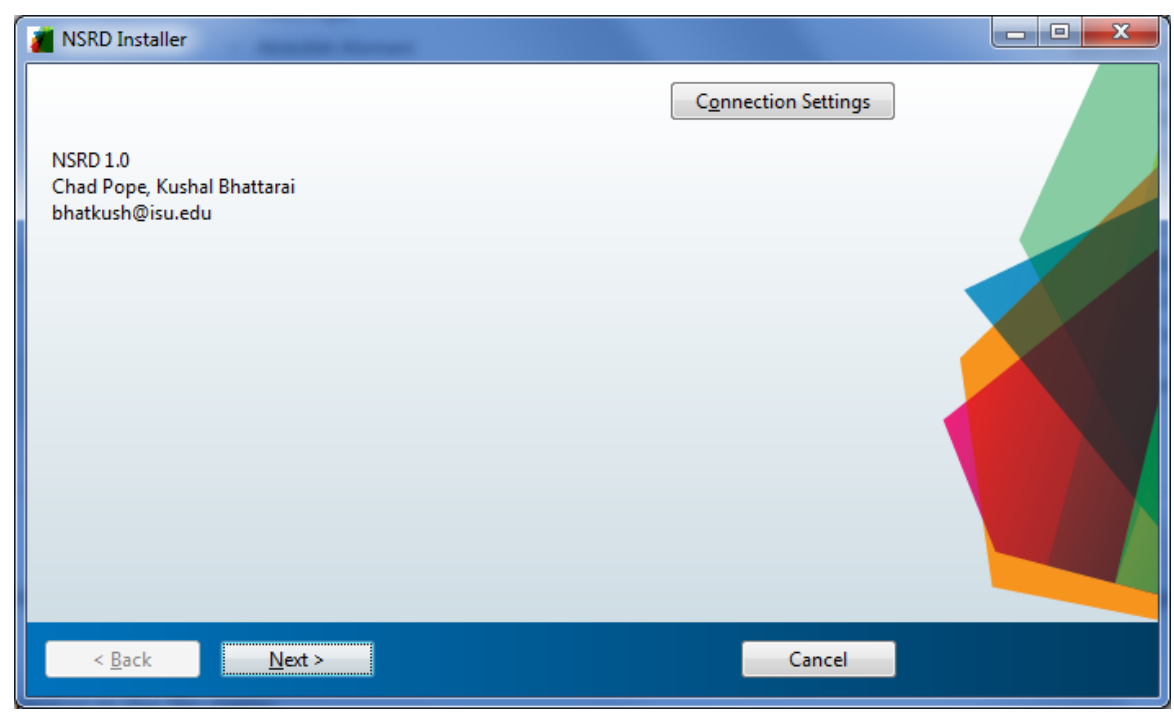

4. Please wait for Contacting Math Works to prepare the installation; then you will see the installation Options window.

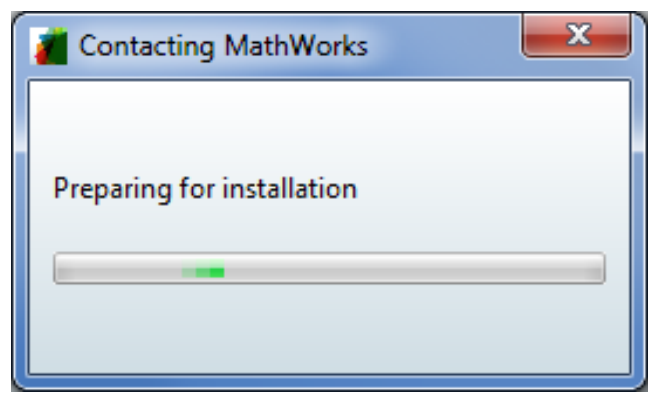

5. Click on the browse button to set a location for the program to be installed.

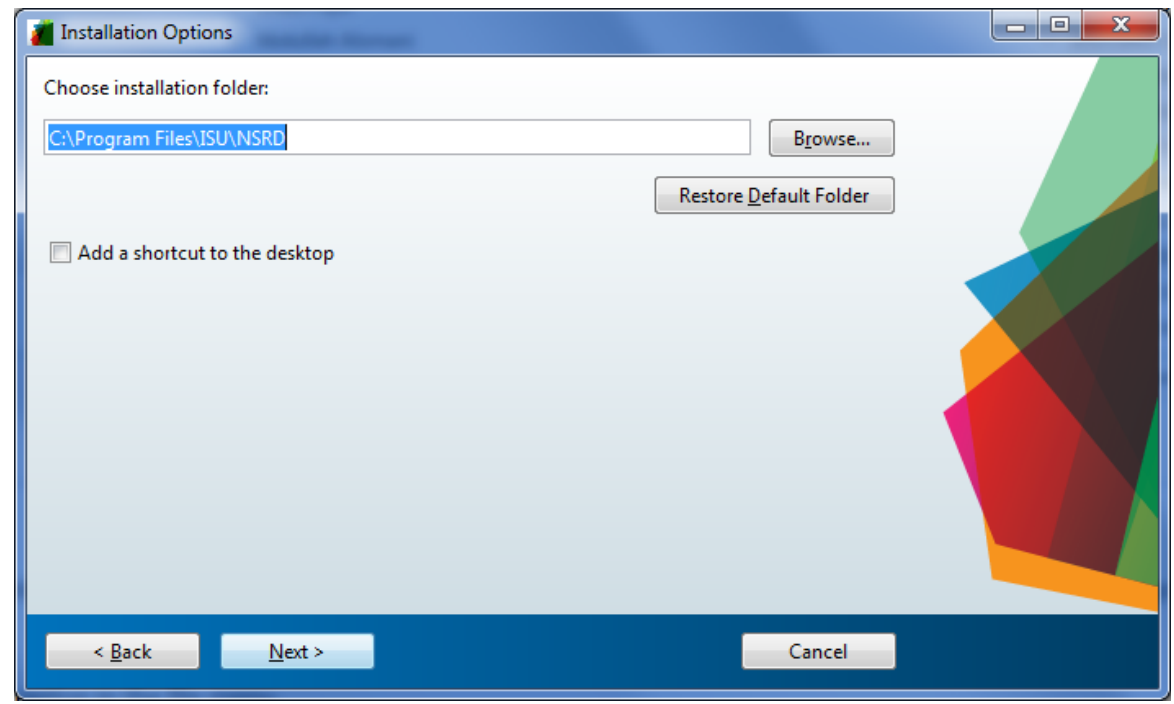


6. Click on the next button to continue installation; then you will see the folder selection.

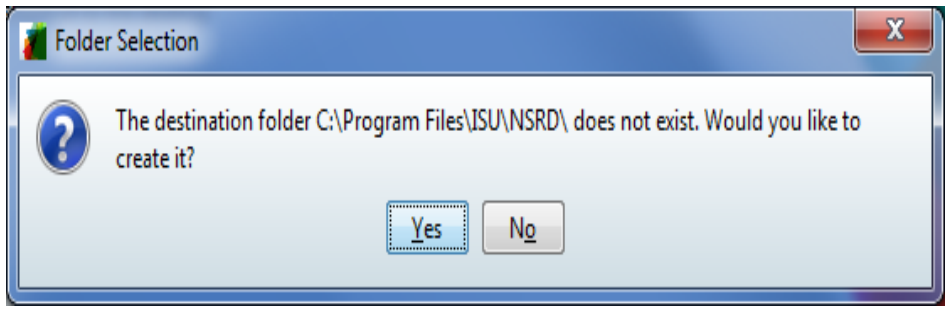

7. Click the Yes or No to either accept or change folders for Installation.

8. Choose an installation folder for the MATLAB compiler runtime.

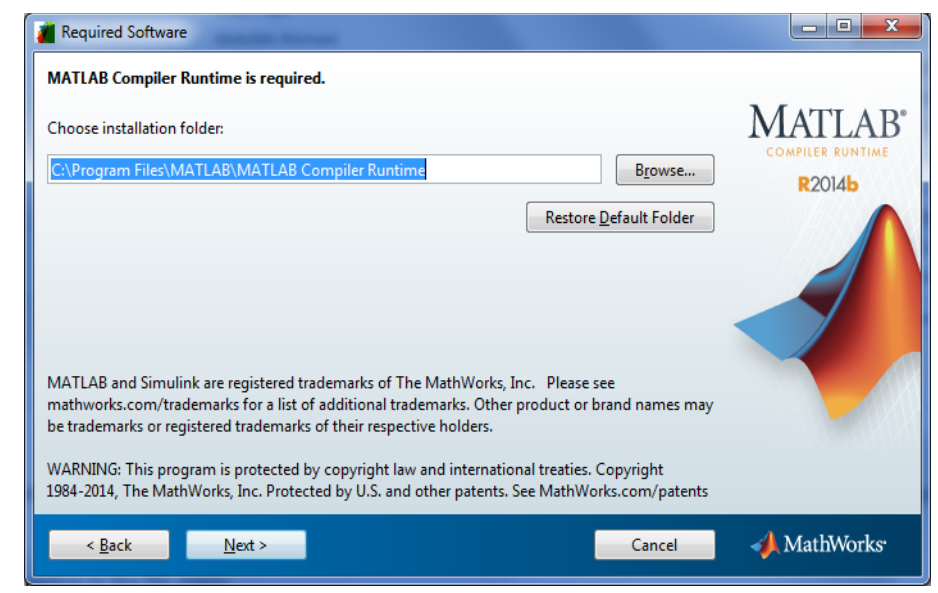

9. Check the Yes or No button to accept the License Agreement. Note: if you check No, the program will cancel.

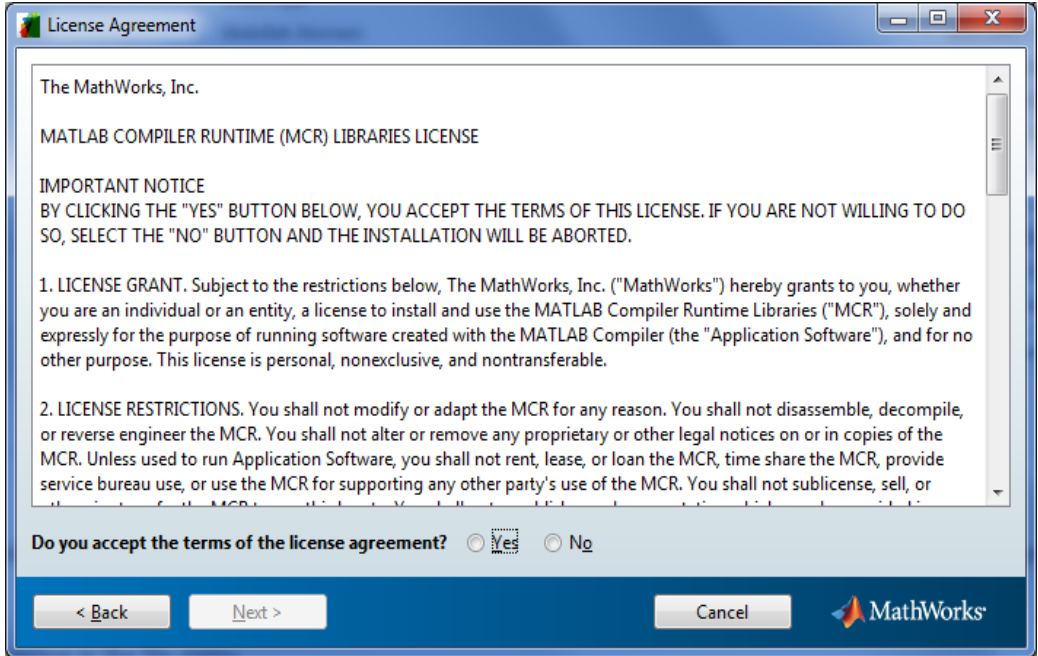


10. Confirmation window will show up; click the install button to start downloading the program.

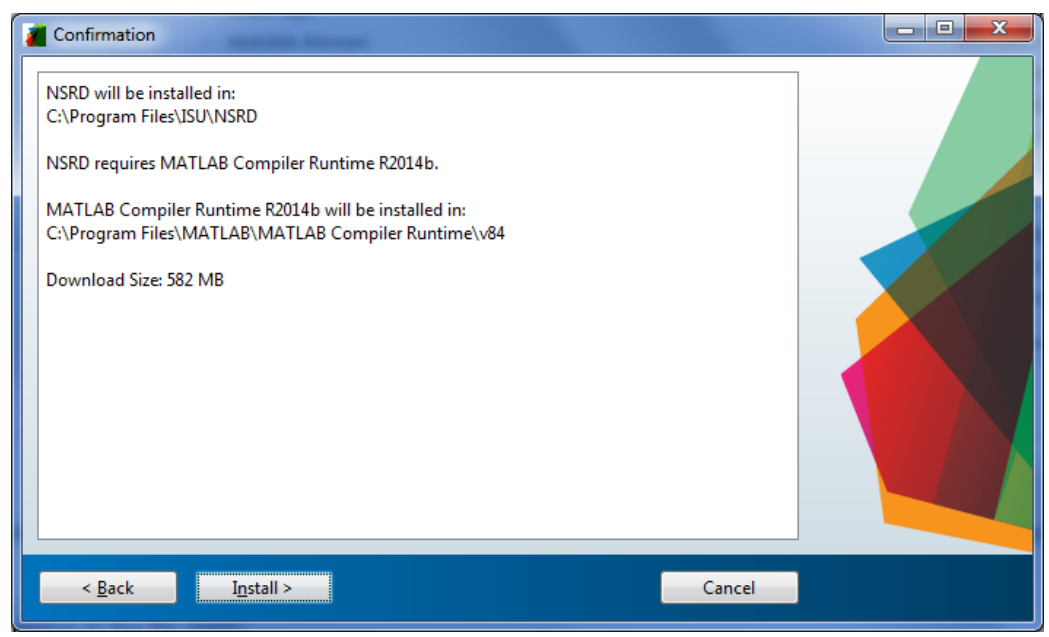

11. The download will keep track of the percentage downloaded $0 \%-100 \%$. If you need to pause the program for any reason. Press the pause button. Once the program reaches $100 \%$ the "installation complete" window will show.

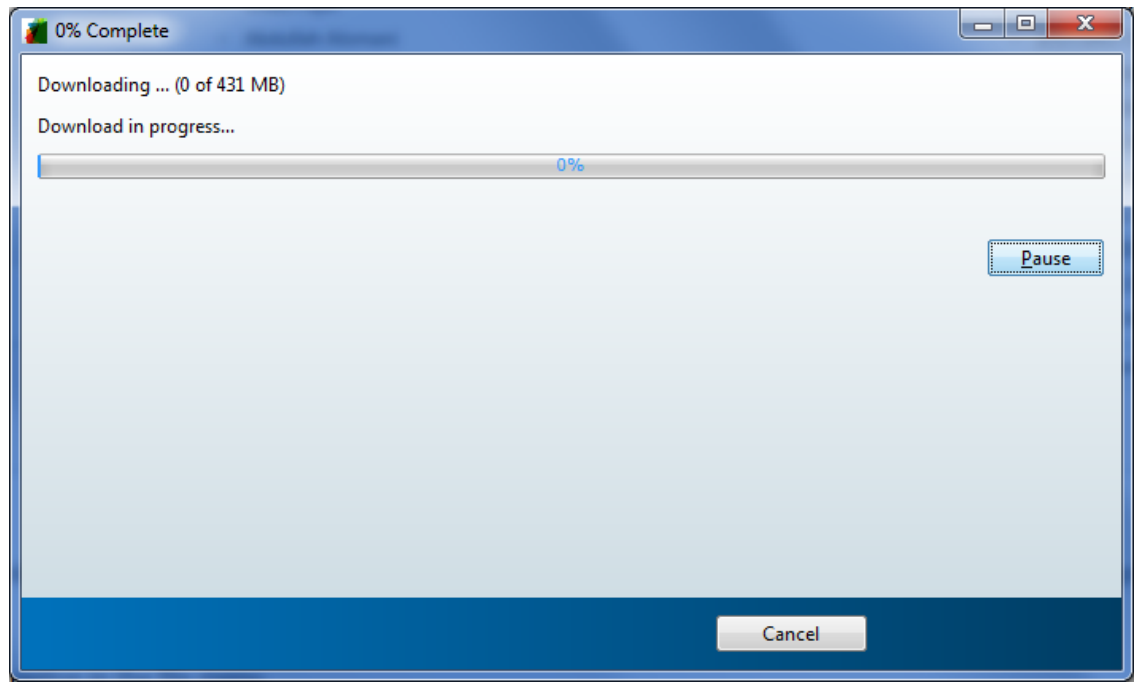


12. The program can be found in several places, Start Menu as pictured; desktop if you chose to allow desktop item; or installation folder that you picked in "Installation options".

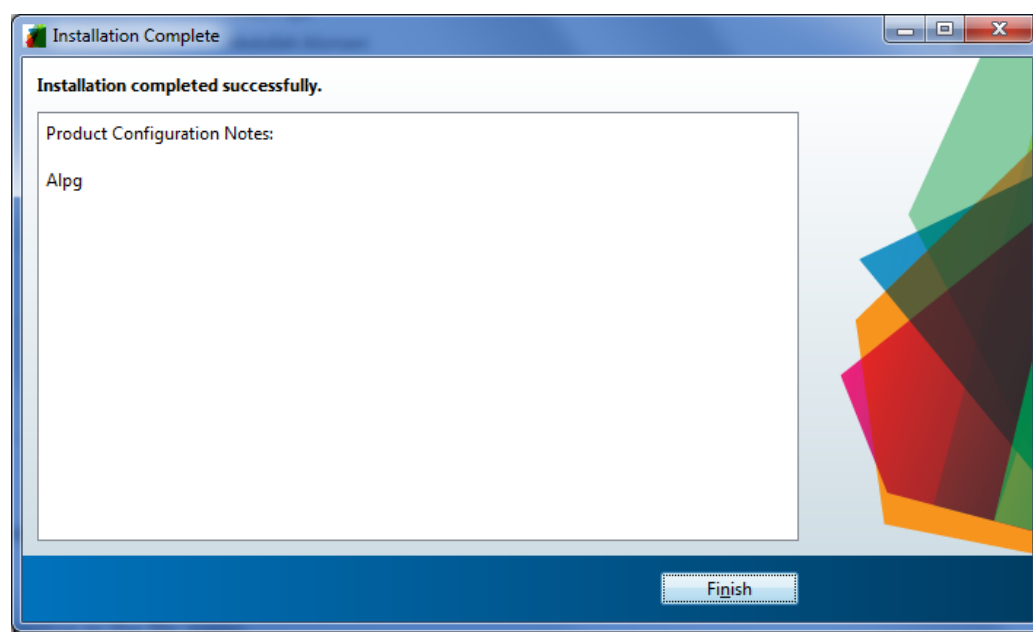

\section{NSRD}

13. Accessories

17. Maintenance

13. Microsoft Office

13. Startup

1. AMD Catalyst Control Center

17. AMD Gaming Evolved

1. World of Warcraft

4 Back

Search programs and files P 


\title{
APPENDIX C: \\ MATLAB Code
}

\begin{abstract}
function varargout $=$ Project 1 (varargin)
\end{abstract}
$\%$ PROJECT1 MATLAB code for Project1.fig

$\% \quad$ PROJECT1, by itself, creates a new PROJECT1 or raises the existing

$\%$ singleton*.

$\%$

$\% \quad H=$ PROJECT1 returns the handle to a new PROJECT1 or the handle to

$\%$ the existing singleton*.

$\%$

$\%$

$\%$

$\%$

$\%$

$\%$

$\%$

$\%$

PROJECT1('CALLBACK',hObject, , ,handles,...) calls the local

function named CALLBACK in PROJECT1.M with the given input arguments.

PROJECT1('Property','Value',...) creates a new PROJECT1 or raises the existing singleton*. Starting from the left, property value pairs are applied to the GUI before Project1_OpeningFcn gets called. An unrecognized property name or invalid value makes property application stop. All inputs are passed to Project1_OpeningFcn via varargin.

*See GUI Options on GUIDE's Tools menu. Choose "GUI allows only one instance to run (singleton)".

$\%$

$\%$ See also: GUIDE, GUIDATA, GUIHANDLES

\% Edit the above text to modify the response to help Project1

\% Last Modified by GUIDE v2.5 10-Apr-2015 14:22:56

$\%$ Begin initialization code - DO NOT EDIT

gui_Singleton $=1$;

gui_State $=$ struct('gui_Name', mfilename, $\ldots$

'gui_Singleton', gui_Singleton, ...

'gui_OpeningFcn', @Project1_OpeningFcn,...

'gui_OutputFcn', @Project1_OutputFcn,...

'gui_LayoutFcn', [], ...

'gui_Callback', []);

if nargin \&\& ischar(varargin $\{1\}$ )

gui_State.gui_Callback = str2func $($ varargin $\{1\})$;

end

if nargout

[varargout $\{1:$ nargout\}] = gui_mainfcn(gui_State, varargin\{:\}); else

gui_mainfcn(gui_State, varargin\{:\});

end

\% End initialization code - DO NOT EDIT

end

$\%$--- Executes just before Project 1 is made visible. 
function Project1_OpeningFcn(hObject, , handles, varargin)

$\%$ This function has no output args, see OutputFcn.

$\%$ hObject handle to figure

$\% \sim$ reserved - to be defined in a future version of MATLAB

$\%$ handles structure with handles and user data (see GUIDATA)

$\%$ varargin command line arguments to Project1 (see VARARGIN)

$\%$ Choose default command line output for Project1

handles.output $=$ hObject;

axes(handles.logo_axes);

imshow('sodaLogo1.png');

$\%$ Update handles structure

guidata(hObject, handles);

\%disable show plot button for all parameter when program starts

set(handles.fit_dist,'Enable','off')

set(handles.mar_pushbutton,'Enable','off');

set(handles.dr_pushbutton,'Enable','off');

set(handles.arf_pushbutton,'Enable','off');

set(handles.rf_pushbutton,'Enable','off');

set(handles.Ipf_pushbutton,'Enable','off');

set(handles.br_pushbutton,'Enable','on');

set(handles.dcf_pushbutton,'Enable','off');

set(handles.cq_pushbutton,'Enable','off');

\%disable text1 all parameter when program starts

set(handles.mar_text1,'Enable','on');

set(handles.mar_popup_dist,'Enable','off');

set(handles.dr_text1,'Enable','off');

set(handles.arf_text1,'Enable','off');

set(handles.rf_text1,'Enable','off');

set(handles.Ipf_text1,'Enable','off');

set(handles.br_text1,'Enable','off');

set(handles.dcf_text1,'Enable','off');

set(handles.cq_text1,'Enable','off');

\%disable text2 for all parameter when program starts

set(handles.mar_text2,'Enable','off');

set(handles.dr_text2,'Enable','off');

set(handles.arf_text2,'Enable','off');

set(handles.rf_text2,'Enable','off');

set(handles.lpf_text2,'Enable','off');

set(handles.dcf_text2,'Enable','off');

\%disable chi/q section distribution input and show plot button

set(handles.windspeed_text1,'Enable','off')

set(handles.windspeed_text2,'Enable','off')

set(handles.cq_pushbutton,'Enable','off')

set(handles.stability_popup,'Enable','off','String',\{'Select Stability'\});

$\%$ set MAR toggle button pressed (to single input) when program starts 
set(handles.mar_togglebutton,'Value',1);

set(handles.mar_togglebutton,'String','Single Input');

set(handles.dcf_popup_dist,'String',\{'Select Distribution';'Normal';...

end

'Beta';'Uniform';'Exponential'\},'Value',1);

$\%$--- Outputs from this function are returned to the command line.

function varargout $=$ Project1_OutputFcn(hObject, $\sim$, handles)

$\%$ varargout cell array for returning output args (see VARARGOUT);

$\%$ hObject handle to figure

$\% \sim$ reserved - to be defined in a future version of MATLAB

$\%$ handles structure with handles and user data (see GUIDATA)

$\%$ Get default command line output from handles structure

varargout $\{1\}=$ handles. output;

end

function num_sample_text_Callback(hObject, , handles)

$\%$ hObject handle to num_sample_text (see GCBO)

$\% \sim$ reserved - to be defined in a future version of MATLAB

$\%$ handles structure with handles and user data (see GUIDATA)

end

$\%$ Hints: get(hObject,'String') returns contents of num_sample_text as text

$\% \quad$ str2double(get(hObject,'String')) returns contents of num_sample_text as a double

$\%$--- Executes during object creation, after setting all properties.

function num_sample_text_CreateFcn(hObject, , handles)

$\%$ hObject handle to num_sample_text (see GCBO)

$\% \sim$ reserved - to be defined in a future version of MATLAB

$\%$ handles empty - handles not created until after all CreateFcns called

$\%$ Hint: edit controls usually have a white background on Windows.

$\% \quad$ See ISPC and COMPUTER.

if ispc \&\& isequal(get(hObject,'BackgroundColor'), get(0,'defaultUicontrolBackgroundColor')) set(hObject,'BackgroundColor','white');

end

end

$\%$--- Executes on button press in rf_pushbutton.

function rf_pushbutton_Callback(hObject, , handles)

$\%$ hObject handle to rf_pushbutton (see GCBO)

$\% \sim$ reserved - to be defined in a future version of MATLAB

$\%$ handles structure with handles and user data (see GUIDATA)

\%when user press RF Show plot button these command are exceuted.

$\%$ grab number from number of samples box.

$\%$ grab number from text1 and text2 box. 
$\%$ grab what kind of distribution user has selected.

$\%$ if normal distribution is selected than generate random normal

$\%$ distribution with given paramter and plot histogram in is axes.

sample = get(handles.num_sample_text,'String');\%grab number from number of sample box samplesize $=$ str2num(sample);

if strcmp(sample,") == 1 || samplesize $<0$

errordlg('Please enter number of samples','Sample Number','modal'); return;

end

col = get(handles.rf_pushbutton,'backg');

set(handles.rf_pushbutton,'str','RUNNING...','backg',[.2 .6 .6]);

pause(eps);

num1 = str2num(get(handles.rf_text1,'String')); \%grab number from number of text box 1

num2 = str2num(get(handles.rf_text2,'String')); \%grab number from number of text box 2

$\%$ Find what kind of distribution user has selected.

contents = get(handles.rf_popup_dist,'String');

popupmenuvalue = contents $\{$ get(handles.rf_popup_dist,'Value')\};

cla(handles.axes 1 ,'reset');

switch popupmenuvalue

case 'Normal'

pd = makedist('Normal','mu',num1,'sigma',num2);

$\mathrm{t}=$ truncate(pd,0,inf);

$\mathrm{n}=\operatorname{random}(\mathrm{t}$, samplesize, 1$)$;

axes(handles.axes1)

nbins $=\max (\min ($ length $(\mathrm{n}) \cdot / 10,100), 50)$;

$x i=$ linspace $(\min (n), \max (n), n b i n s)$;

$\mathrm{dx}=\operatorname{mean}(\operatorname{diff}(\mathrm{xi}))$;

$\mathrm{fi}=\operatorname{histc}(\mathrm{n}, \mathrm{xi}-\mathrm{dx})$;

$\mathrm{fi}=\mathrm{fi} . / \mathrm{sum}(\mathrm{fi}) . / \mathrm{dx}$;

assignin('base','rfxi', xi);

assignin('base','rffi2', fi);

bar(xi,fi,'FaceColor',[.2 .6 .6],'EdgeColor',[.2 .6 .6], 'BarWidth',1);

axis tight;

$\% \quad$ hist $(n, 50)$;

ylabel('Probability Density');

xlabel('RF');

title(['Respirable Fraction distribution plot with Normal Distribution Imu ='...

num2str(mean(n)) ' and Isigma = ' num2str(std(n))])

case 'Beta'

pd = makedist('Beta','a',num1,'b', num2);

$\mathrm{t}=$ truncate(pd,0,inf);

$\mathrm{n}=$ random(t,samplesize, 1$)$;

axes(handles.axes1)

nbins $=\max (\min ($ length $(n) . / 10,100), 50)$;

$x i=$ linspace $(\min (n), \max (n), n b i n s)$;

$\mathrm{dx}=\operatorname{mean}(\operatorname{diff}(\mathrm{xi}))$;

$\mathrm{fi}=\operatorname{histc}(\mathrm{n}, \mathrm{xi}-\mathrm{dx})$;

$\mathrm{fi}=\mathrm{fi} . / \mathrm{sum}(\mathrm{fi}) . / \mathrm{dx}$;

assignin('base','rfxi', xi);

assignin('base','rffi2', fi); 


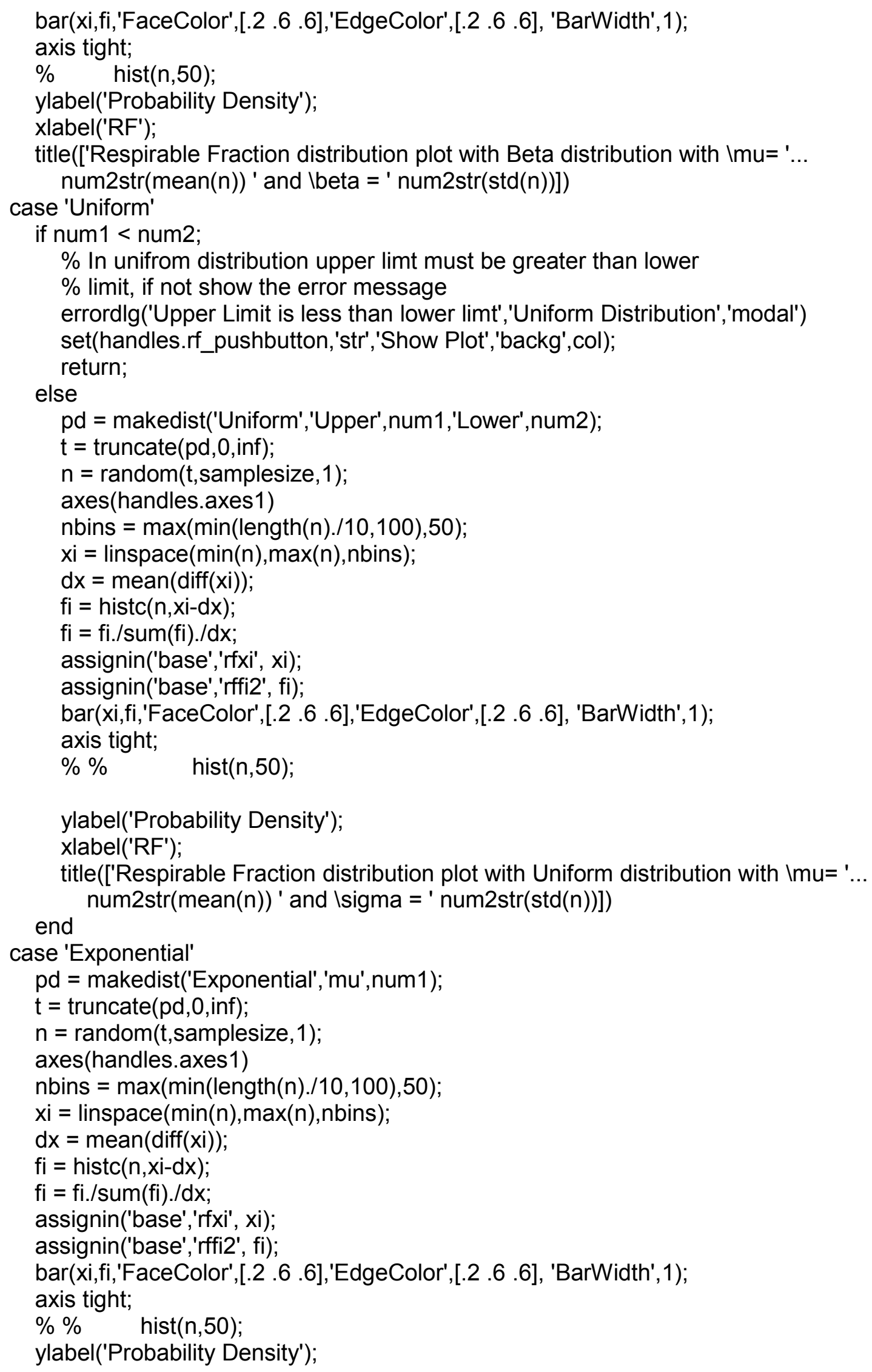


xlabel('RF');

title(['Respirable Fraction distribution plot with Exponential distribution with $\mid m u='$ '...

end num2str(mean(n)) ' and Isigma = ' num2str(std(n))])

set(handles.rf_pushbutton,'str','Show Plot','backg',col);

end

function rf_text2_Callback(hObject, , handles)

$\%$ hObject handle to if_text2 (see GCBO)

$\% \sim$ reserved - to be defined in a future version of MATLAB

$\%$ handles structure with handles and user data (see GUIDATA)

end

$\%$ Hints: get(hObject,'String') returns contents of rf_text2 as text

$\% \quad$ str2double(get(hObject,'String')) returns contents of rf_text2 as a double

$\%$--- Executes during object creation, after setting all properties.

function rf_text2_CreateFcn(hObject, , handles)

$\%$ hObject handle to if text2 (see GCBO)

$\% \sim$ reserved - to be defined in a future version of MATLAB

$\%$ handles empty - handles not created until after all CreateFcns called

$\%$ Hint: edit controls usually have a white background on Windows.

$\% \quad$ See ISPC and COMPUTER.

if ispc \&\& isequal(get(hObject,'BackgroundColor'), get(0,'defaultUicontrolBackgroundColor')) set(hObject,'BackgroundColor','white');

end

end

function rf_text1_Callback(hObject, , handles)

$\%$ hObject handle to rf_text1 (see GCBO)

$\% \sim$ reserved - to be defined in a future version of MATLAB

$\%$ handles structure with handles and user data (see GUIDATA)

end

$\%$ Hints: get(hObject,'String') returns contents of rf_text1 as text

$\% \quad$ str2double(get(hObject,'String')) returns contents of rf_text1 as a double

$\%$--- Executes during object creation, after setting all properties.

function rf_text1_CreateFcn(hObject, , handles)

$\%$ hObject handle to if_text1 (see GCBO)

$\% \sim$ reserved - to be defined in a future version of MATLAB

$\%$ handles empty - handles not created until after all CreateFcns called

$\%$ Hint: edit controls usually have a white background on Windows.

$\% \quad$ See ISPC and COMPUTER.

if ispc \&\& isequal(get(hObject,'BackgroundColor'), get(0,'defaultUicontrolBackgroundColor')) 


$$
\text { set(hObject,'BackgroundColor','white'); }
$$

end

end

$\%$--- Executes on selection change in rf_popup_dist.

function rf_popup_dist_Callback(hObject, $\sim$, handles)

$\%$ hObject handle to rf_popup_dist (see GCBO)

$\% \sim$ reserved - to be defined in a future version of MATLAB

$\%$ handles structure with handles and user data (see GUIDATA)

$\%$ This function is executes when a Respirable fraction popup menu distribution $\%$ is choosen.

$\%$ find the stiring the user have selected. contents = cellstr(get(hObject,'String'));

rfpopchoice $=$ contents $\{$ get $($ hObject,, Value' $)\}$;

switch rfpopchoice case 'Normal'

\%if normal is selected enable input text box and also display parameter $\%$ required in those text box.

set(handles.rf_text1,'Enable','inactive') set(handles.rf_text2,'Enable','inactive') set(handles.rf_pushbutton,'Enable','on') set(handles.rf_text1,'String','Mean'); set(handles.rf_text2,'String','Std Deviation'); set(handles.rf_text1,'TooltipString',") set(handles.rf_text2,'TooltipString',") case 'Beta'

\%if Beta is selected enable input text box and also display parameter \%required in those text box. set(handles.rf_text1,'Enable','inactive') set(handles.rf_text2,'Enable','inactive') set(handles.rf_pushbutton,'Enable','on') set(handles.rf_text1,'String','a'); set(handles.ff_text2,'String','b'); set(handles.rf_text1,'TooltipString','shape parameter') set(handles.rf_text2,'TooltipString','shape parameter') case 'Uniform'

\%if Uniform is selected enable input text box and also display parameter $\%$ required in those text box.

set(handles.rf_text1,'Enable','inactive') set(handles.rf_text2,'Enable','inactive') set(handles.rf_pushbutton,'Enable','on') set(handles.rf_text1,'String','Upper Limit'); set(handles.rf_text2,'String','Lower Limit'); set(handles.rf_text1,'TooltipString',") set(handles.rf_text2,'TooltipString',") case 'Exponential'

\%if Exponential is selected enable input text box and also display parameter \%required in those text box. 


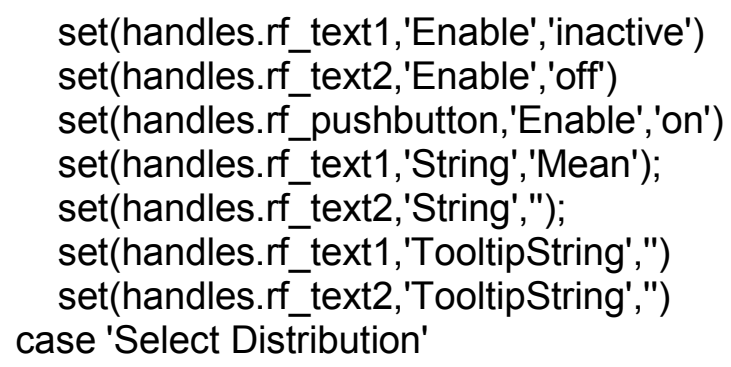

end

$\%$ Hints: contents = cellstr(get(hObject,'String')) returns rf_popup_dist contents as cell array

$\% \quad$ contents\{get(hObject,'Value')\} returns selected item from rf_popup_dist

$\%$--- Executes during object creation, after setting all properties.

function rf_popup_dist_CreateFcn(hObject, , handles)

$\%$ hObject handle to if_popup_dist (see GCBO)

$\% \sim$ reserved - to be defined in a future version of MATLAB

$\%$ handles empty - handles not created until after all CreateFcns called

$\%$ Hint: popupmenu controls usually have a white background on Windows.

$\% \quad$ See ISPC and COMPUTER.

if ispc \&\& isequal(get(hObject,'BackgroundColor'), get( 0 ,'defaultUicontrolBackgroundColor')) set(hObject,'BackgroundColor','white'); end

end

$\%$--- Executes on selection change in dr_popup_dist.

function dr_popup_dist_Callback(hObject, $\sim$, handles)

$\%$ hObject handle to dr_popup_dist (see GCBO)

$\% \sim$ reserved - to be defined in a future version of MATLAB

$\%$ handles structure with handles and user data (see GUIDATA)

contents $=$ cellstr(get(hObject,'String'));

drpopchoice $=$ contents $\{$ get $($ hObject, 'Value') $\}$;

switch drpopchoice

case 'Normal'

set(handles.dr_text1,'Enable','inactive')

set(handles.dr_text2,'Enable','inactive')

set(handles.dr_pushbutton,'Enable','on')

set(handles.dr_text1,'String','Mean');

set(handles.dr_text2,'String','Std Deviation'); 


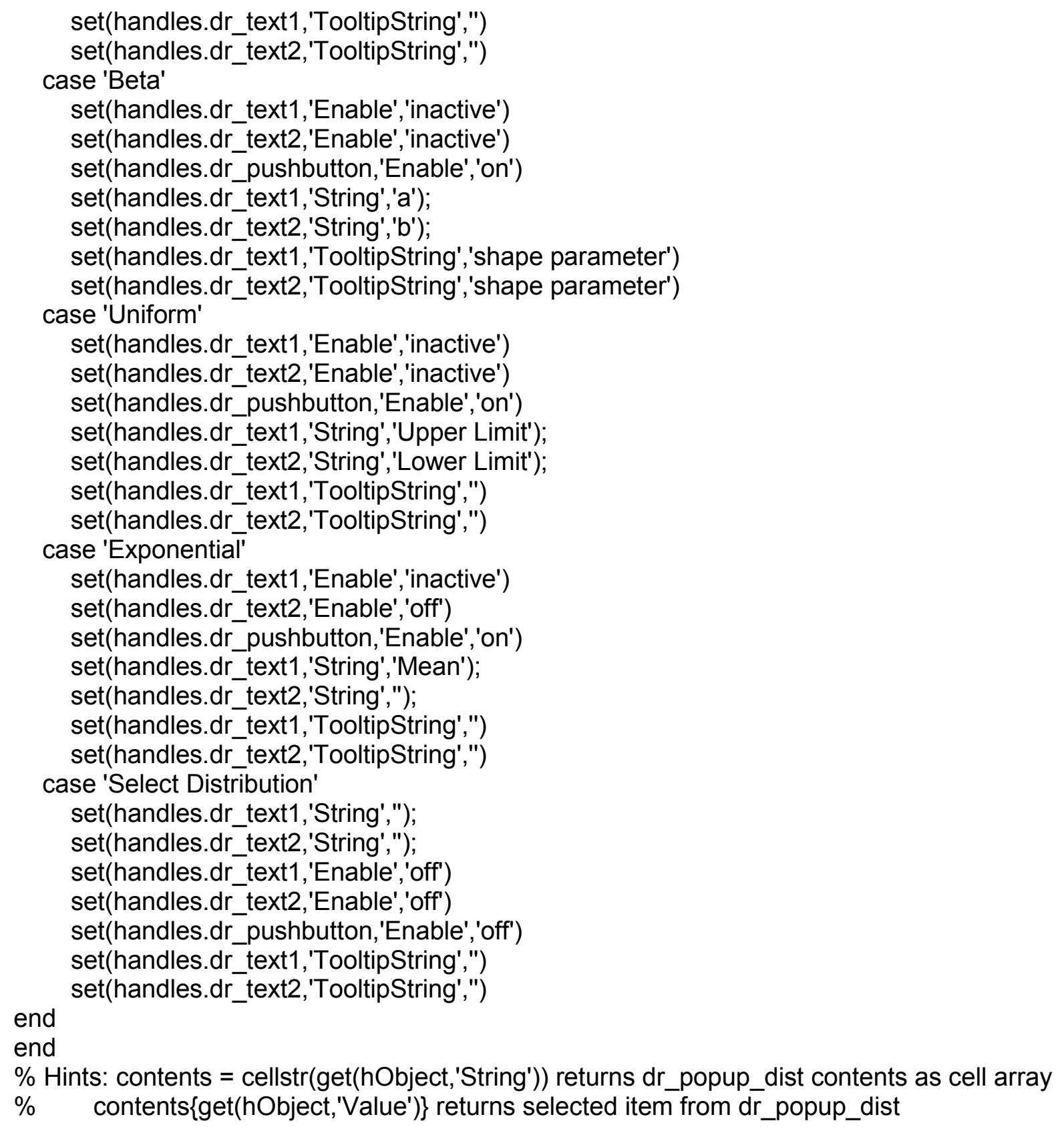

$\%$--- Executes during object creation, after setting all properties.

function dr_popup_dist_CreateFcn(hObject, , handles)

$\%$ hObject handle to $\overline{\mathrm{d}} \mathrm{r}$ _popup_dist (see GCBO)

$\% \sim$ reserved - to be defined in a future version of MATLAB

$\%$ handles empty - handles not created until after all CreateFcns called

\% Hint: popupmenu controls usually have a white background on Windows.

$\% \quad$ See ISPC and COMPUTER.

if ispc \&\& isequal(get(hObject,'BackgroundColor'), get( 0 ,'defaultUicontrolBackgroundColor')) end 
end

function dr_text1_Callback(hObject, , handles)

$\%$ hObject handle to dr_text1 (see GCBO)

$\% \sim$ reserved - to be defined in a future version of MATLAB

$\%$ handles structure with handles and user data (see GUIDATA)

$\%$ Hints: get(hObject,'String') returns contents of dr_text1 as text

$\% \quad$ str2double(get(hObject,'String')) returns contents of dr_text1 as a double

end

$\%$--- Executes during object creation, after setting all properties.

function dr_text1_CreateFcn(hObject, , handles)

$\%$ hObject handle to dr_text1 (see GCBO)

$\%$ reserved - to be defined in a future version of MATLAB

$\%$ handles empty - handles not created until after all CreateFcns called

\% Hint: edit controls usually have a white background on Windows.

$\% \quad$ See ISPC and COMPUTER.

if ispc \&\& isequal(get(hObject,'BackgroundColor'), get( 0 ,'defaultUicontrolBackgroundColor')) set(hObject,'BackgroundColor','white');

end

end

function dr_text2_Callback(hObject, $\sim$, handles)

$\%$ hObject handle to dr_text2 (see GCBO)

$\% \sim$ reserved - to be defined in a future version of MATLAB

$\%$ handles structure with handles and user data (see GUIDATA)

$\%$ Hints: get(hObject,'String') returns contents of dr_text2 as text

$\% \quad$ str2double(get(hObject,'String')) returns contents of dr_text2 as a double

end

$\%$--- Executes during object creation, after setting all properties.

function dr_text2_CreateFcn(hObject, , handles)

$\%$ hObject handle to dr_text2 (see GCBO)

$\% \sim$ reserved - to be defined in a future version of MATLAB

$\%$ handles empty - handles not created until after all CreateFcns called

$\%$ Hint: edit controls usually have a white background on Windows.

$\% \quad$ See ISPC and COMPUTER.

if ispc \&\& isequal(get(hObject,'BackgroundColor'), get(0,'defaultUicontrolBackgroundColor')) set(hObject,'BackgroundColor','white');

end

end

$\%$--- Executes on button press in dr_pushbutton.

function dr_pushbutton_Callback(hObject, , handles)

$\%$ hObject handle to $\overline{\mathrm{d}}$ __pushbutton (see GCBO)

$\% \sim$ reserved - to be defined in a future version of MATLAB 
$\%$ handles structure with handles and user data (see GUIDATA) sample = get(handles.num_sample_text,'String');

samplesize $=$ str2num(sample);

if strcmp(sample,") == 1 || samplesize $<0$

errordlg('Please enter number of samples','Sample Number','modal'); return;

end

col = get(handles.dr_pushbutton,'backg');

set(handles.dr_pushbutton,'str','RUNNING...','backg',[.2 .6 .6]);

pause(eps);

num1 = str2num(get(handles.dr_text1,'String'));

num2 = str2num(get(handles.dr_text2,'String'));

contents = get(handles.dr_popup_dist,'String');

popupmenuvalue $=$ contents $\{$ get(handles.dr_popup_dist,'Value')\};

cla(handles.axes1,'reset');

switch popupmenuvalue

case 'Normal'

pd = makedist('Normal','mu',num1,'sigma',num2);

$\mathrm{t}=$ truncate $(\mathrm{pd}, 0.1,1)$;

$\mathrm{n}=$ random(t,samplesize, 1$)$;

axes(handles.axes1)

nbins $=\max (\min ($ length $(\mathrm{n}) \cdot / 10,100), 50)$;

$x i=$ linspace $(\min (n), \max (n), n b i n s)$;

$\mathrm{dx}=\operatorname{mean}(\operatorname{diff}(\mathrm{xi}))$;

$\mathrm{fi}=\operatorname{histc}(\mathrm{n}, \mathrm{xi}-\mathrm{dx})$;

$\mathrm{fi}=\mathrm{fi} . / \mathrm{sum}(\mathrm{fi}) . / \mathrm{dx}$;

assignin('base','drxi', xi);

assignin('base','drfi2', fi);

bar(xi,fi,'FaceColor',[.2 .6 .6],'EdgeColor',[.2 .6 .6], 'BarWidth',1);

axis tight;

$\% \quad h i s t(n, 50)$;

axis tight;

ylabel('Probability Density');

xlabel('DR');

title(['DR distribution plot with Normal distribution'...

'Imu=' num2str(mean(n)) ' and Isigma = ' num2str(std(n))])

case 'Beta'

pd = makedist('Beta','a',num1,'b',num2);

$\mathrm{t}=$ truncate $(\mathrm{pd}, 0.1,1)$

$\mathrm{n}=$ random(t,samplesize, 1$)$;

axes(handles.axes1)

nbins $=\max (\min ($ length $(\mathrm{n}) \cdot / 10,100), 50)$;

$x i=$ linspace $(\min (n), \max (n), n b i n s)$;

$\mathrm{dx}=\operatorname{mean}(\operatorname{diff}(\mathrm{xi}))$;

$\mathrm{fi}=\operatorname{histc}(\mathrm{n}, \mathrm{xi}-\mathrm{dx})$;

$\mathrm{fi}=\mathrm{fi} . / \mathrm{sum}(\mathrm{fi}) . / \mathrm{dx}$;

assignin('base','drxi', xi);

assignin('base','drfi2', fi);

bar(xi,fi,'FaceColor',[.2 .6 .6],'EdgeColor',[.2 .6 .6], 'BarWidth',1);

axis tight;

$\% \quad \operatorname{hist}(\mathrm{n}, 50)$; 


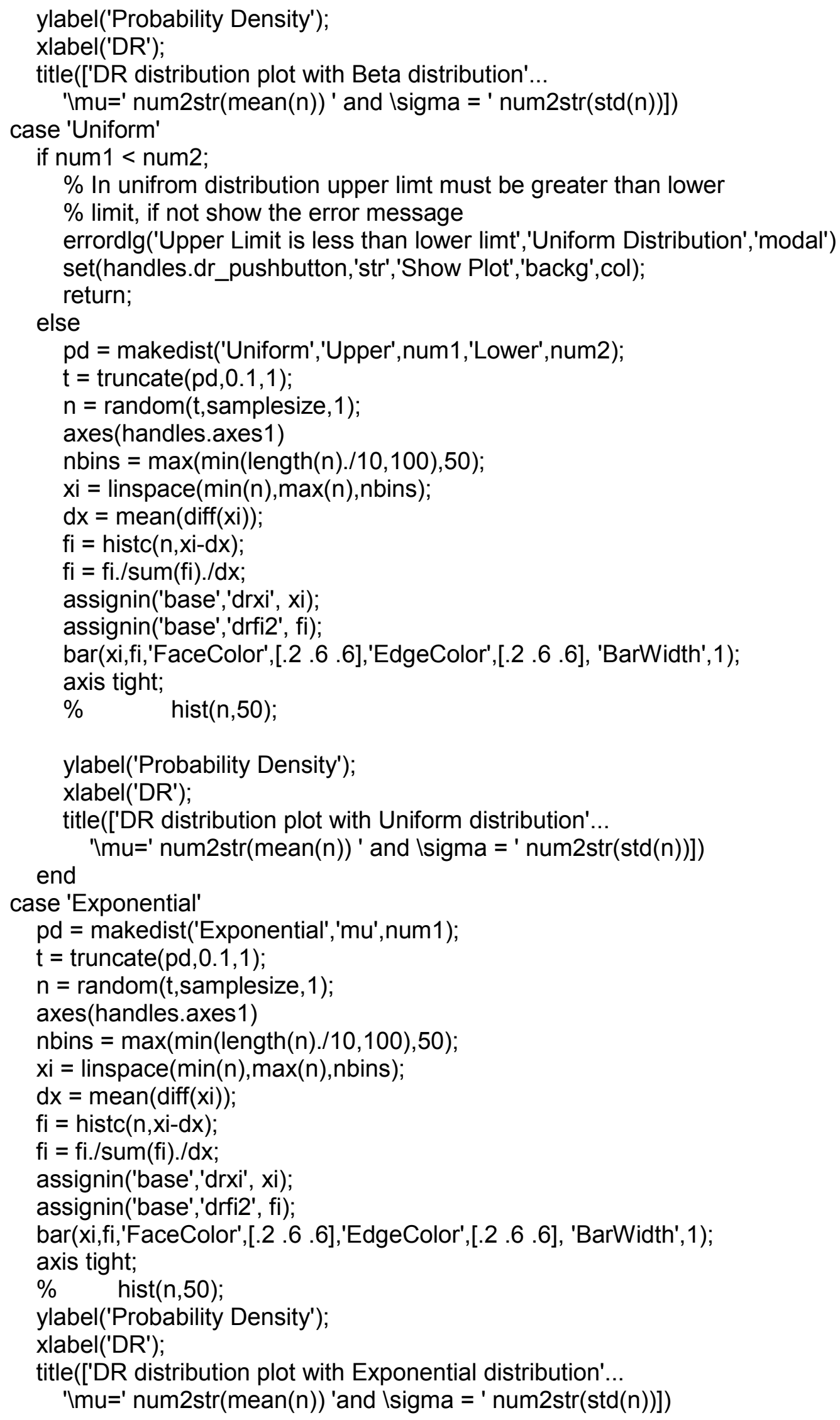


end

set(handles.dr_pushbutton,'str','Show Plot','backg',col);

end

$\%$--- Executes on button press in run_pushbutton.

function run_pushbutton_Callback(hObject, , handles)

$\%$ hObject handle to run_pushbutton (see GCBO)

$\% \sim$ reserved - to be defined in a future version of MATLAB

$\%$ handles structure with handles and user data (see GUIDATA)

sample = get(handles.num_sample_text,'String');

samplesize $=$ str2num(sample);

col = get(handles.run_pushbutton,'backg');

set(handles.run_pushbutton,'str','RUNNING...','backg',[.2 .6 .6]);

pause(eps);

a = get(handles.mar_togglebutton,'Value');

b = get(handles.dr_togglebutton,'Value');

c = get(handles.arf_togglebutton,'Value');

$\mathrm{d}=$ get(handles.rf_togglebutton,'Value');

e = get(handles.lpf_togglebutton,'Value');

$\mathrm{f}=$ get(handles.br_togglebutton,'Value');

$\mathrm{g}=$ get(handles.dcf_togglebutton,'Value');

$\mathrm{h}=$ get(handles.cq_togglebutton,'Value');

if $a==0\|b==0\| c==0\|d==0\| e==0\|f==0\| g==0 \| h==0$;

if strcmp(sample,") == 1 || samplesize $<0$

errordlg('Please enter number of samples','Sample Number','modal');

set(handles.run_pushbutton,'str','Show Plot','backg',col);

return;

end

end

\%compute material at risk

if $\mathrm{a}=\mathbf{=} 0$;

num1 = str2num(get(handles.mar_text1,'String'));

num2 = str2num(get(handles.mar_text2,'String'));

contents = get(handles.mar_popup_dist,'String');

popupmenuvalue = contents $\{$ get(handles.mar_popup_dist,'Value')\};

switch popupmenuvalue

case 'Normal'

pd = makedist('Normal','mu',num1,'sigma',num2);

$\mathrm{t}=$ truncate(pd,0,inf);

mar = random $(\mathrm{t}$, samplesize, 1$)$;

case 'Beta'

pd = makedist('Beta','a',num1,'b',num2);

$\mathrm{t}=$ truncate $(\mathrm{pd}, 0$,inf);

mar $=$ random $(\mathrm{t}$, samplesize, 1$)$;

case 'Uniform'

if num1 < num2;

$\%$ In unifrom distribution upper limt must be greater than lower

$\%$ limit, if not show the error message

errordlg('Upper Limit is less than lower limt','Uniform Distribution','modal') 


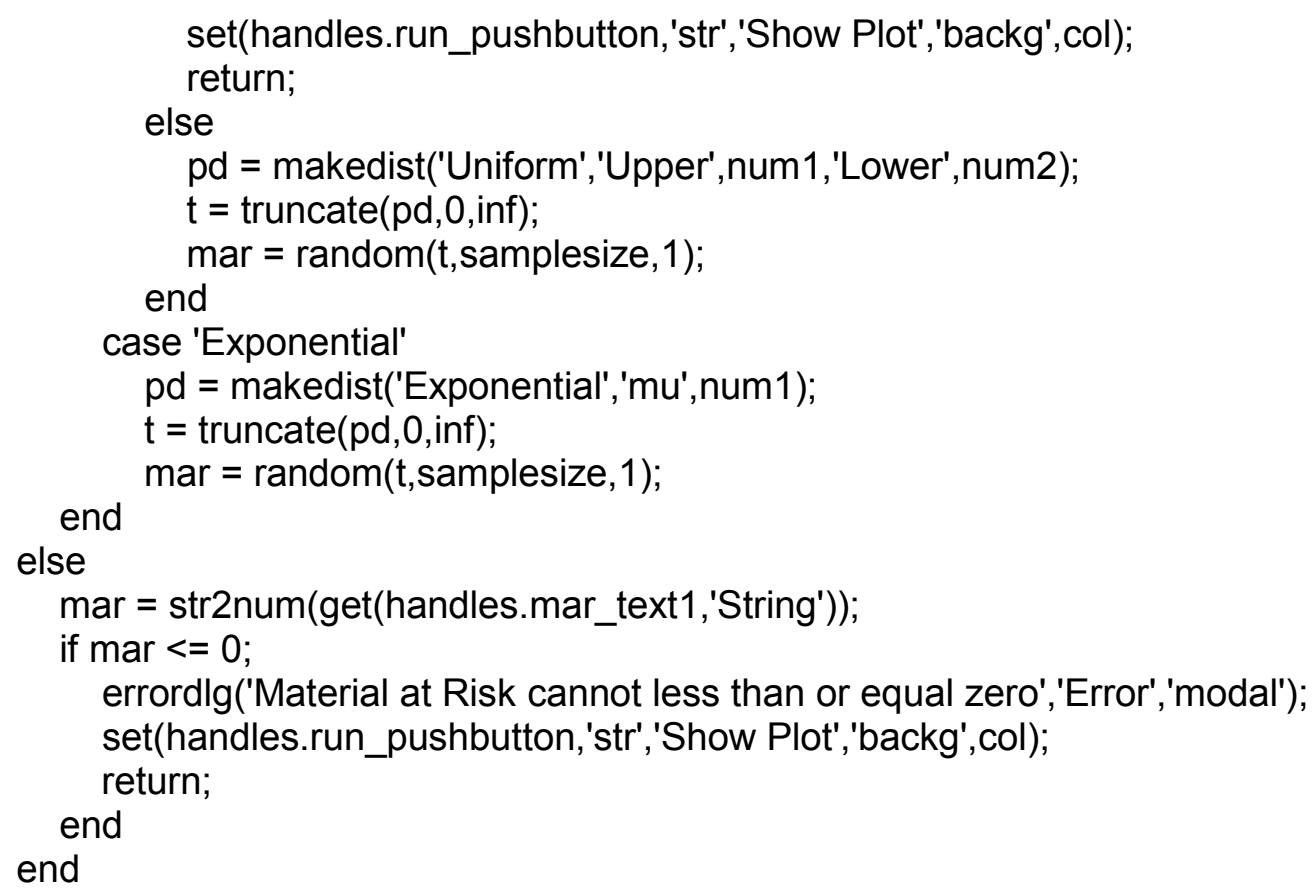




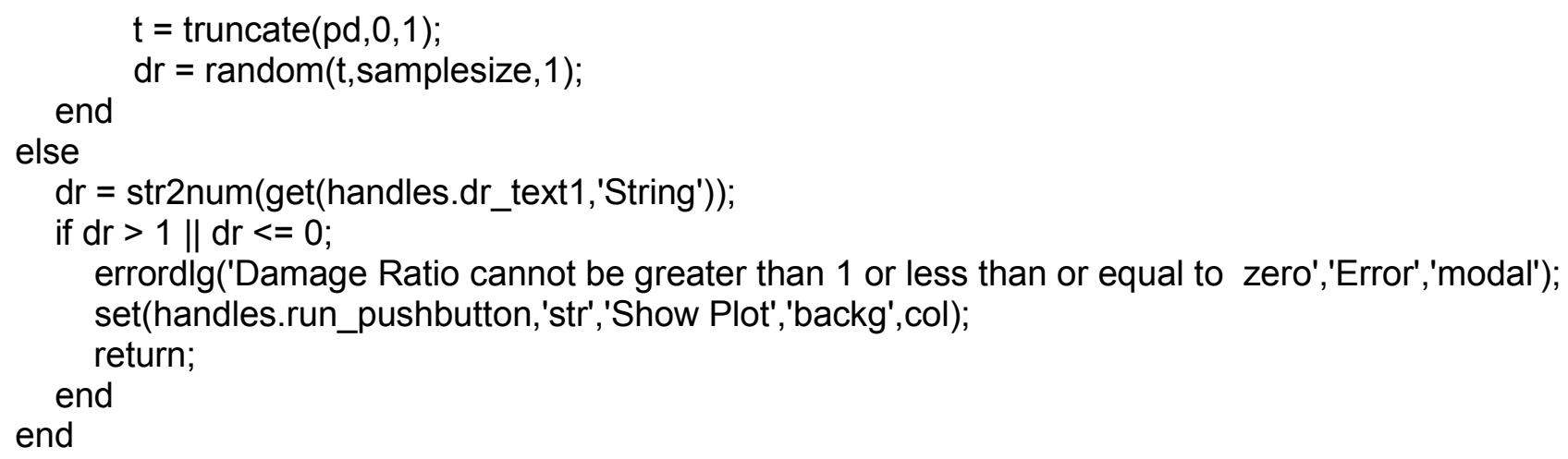




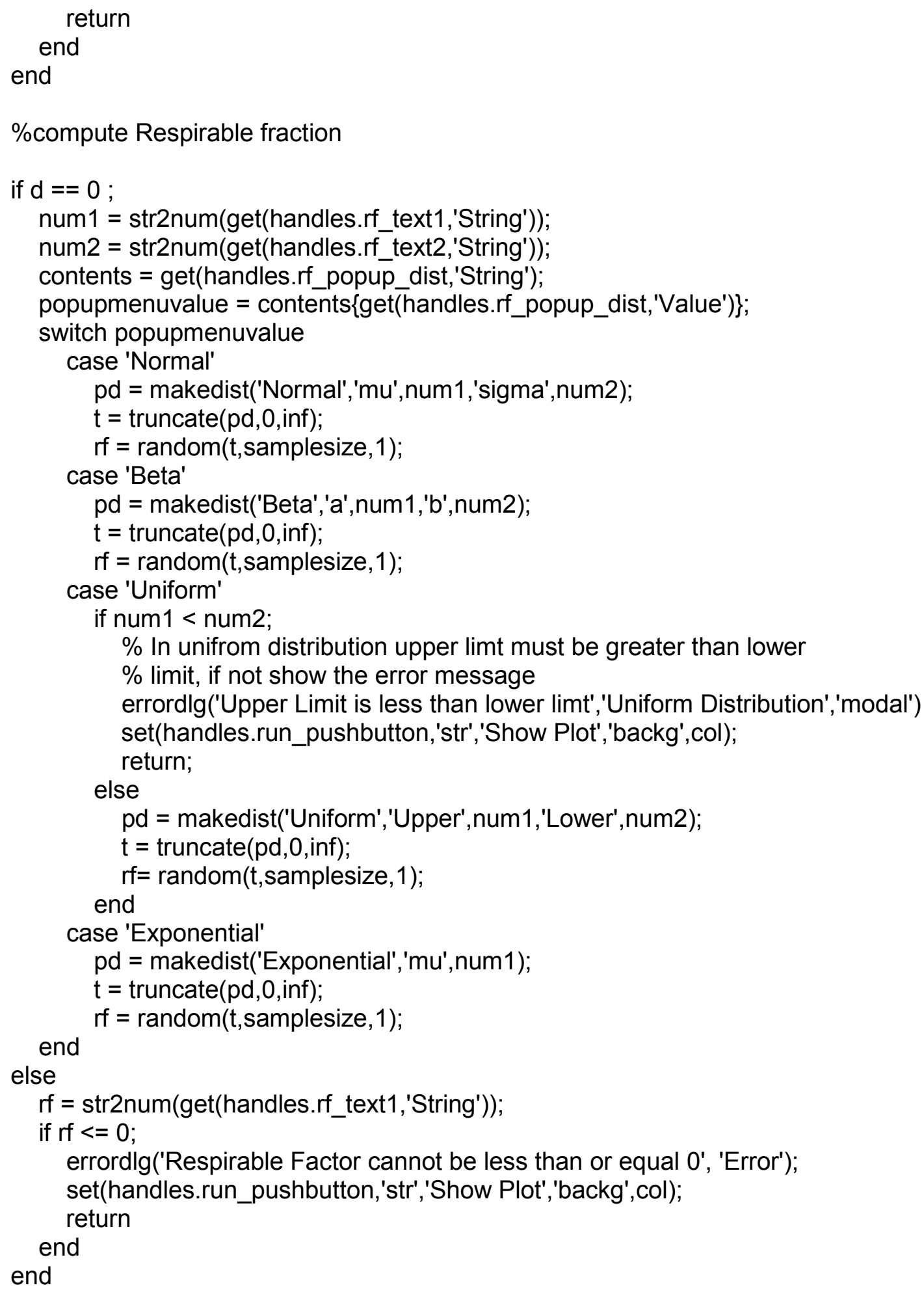




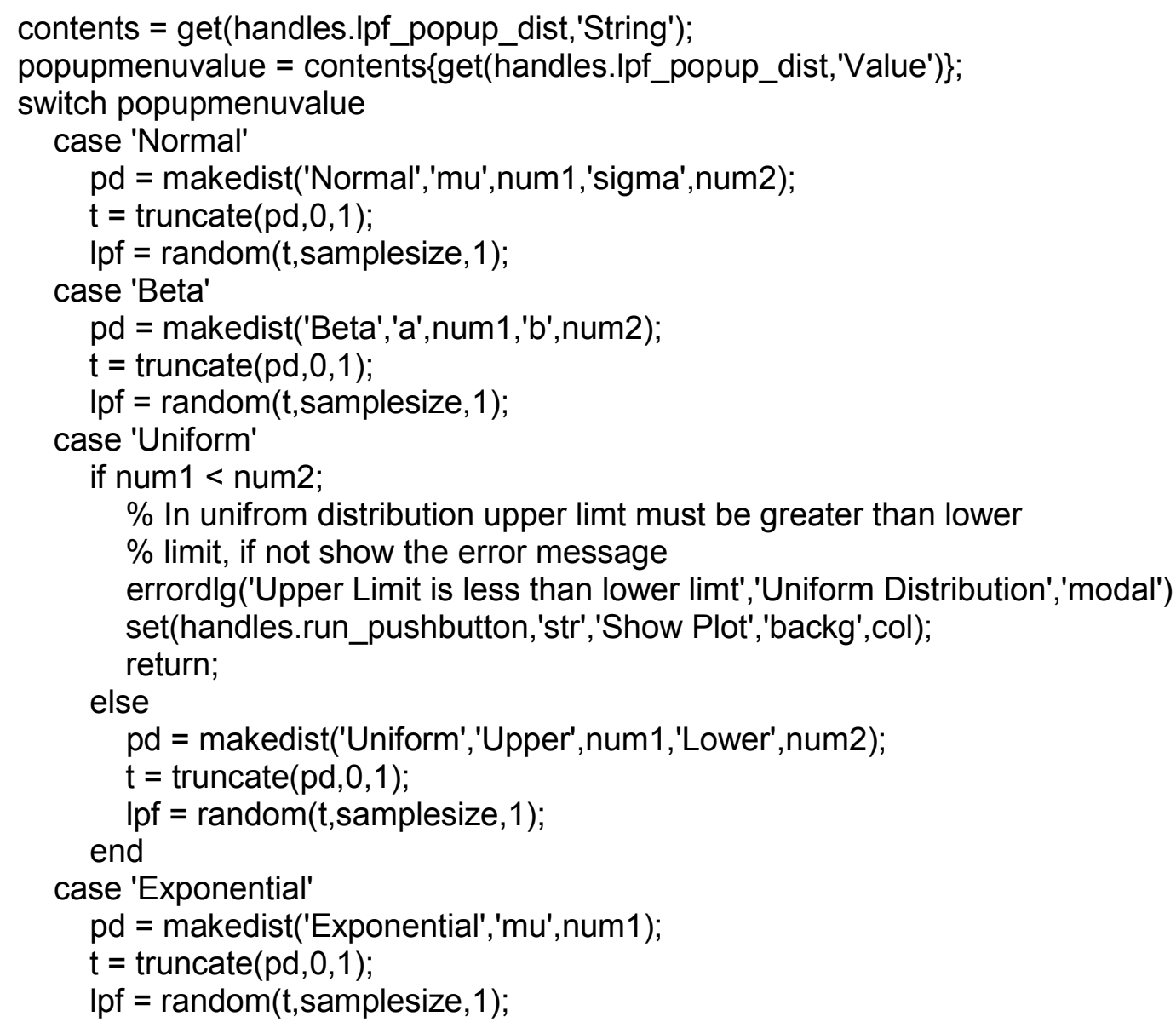




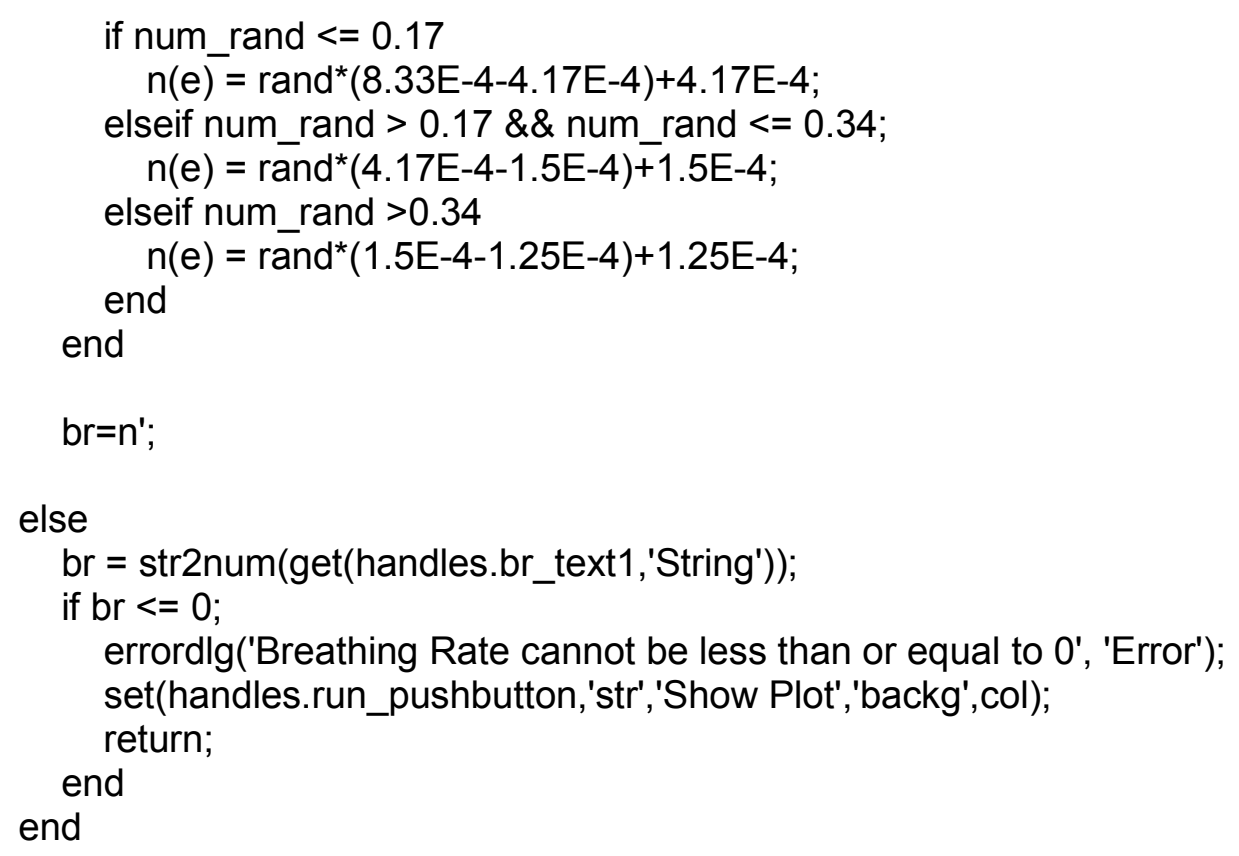

\%compute dose conversion factor

if $\mathrm{g}==0$;

num1 = str2num(get(handles.dcf_text1,'String'));

num2 = str2num(get(handles.dcf_text2,'String'));

contents = get(handles.dcf_popup_dist,'String');

popupmenuvalue = contents\{get(handles.dcf_popup_dist,'Value')\};

switch popupmenuvalue

case 'Normal'

pd = makedist('Normal','mu',num1,'sigma',num2);

$\mathrm{t}=$ truncate $(\mathrm{pd}, 0$,inf);

$\mathrm{dcf}=$ random(t,samplesize, 1$)$;

case 'Beta'

pd = makedist('Beta','a',num1,'b',num2);

$\mathrm{t}=$ truncate $(\mathrm{pd}, 0$,inf);

$\mathrm{dcf}=$ random $(\mathrm{t}$, samplesize, 1$)$;

case 'Uniform'

if num1 < num2;

$\%$ In unifrom distribution upper limt must be greater than lower

$\%$ limit, if not show the error message

errordlg('Upper Limit is less than lower limt','Uniform Distribution','modal')

set(handles.run_pushbutton,'str','Show Plot','backg',col);

return;

else

pd = makedist('Uniform','Upper',num1,'Lower',num2);

$\mathrm{t}=$ truncate $(\mathrm{pd}, 0$,inf);

$\mathrm{dcf}=\operatorname{random}(\mathrm{t}$, samplesize, 1$)$;

end

case 'Exponential'

Appendix C 


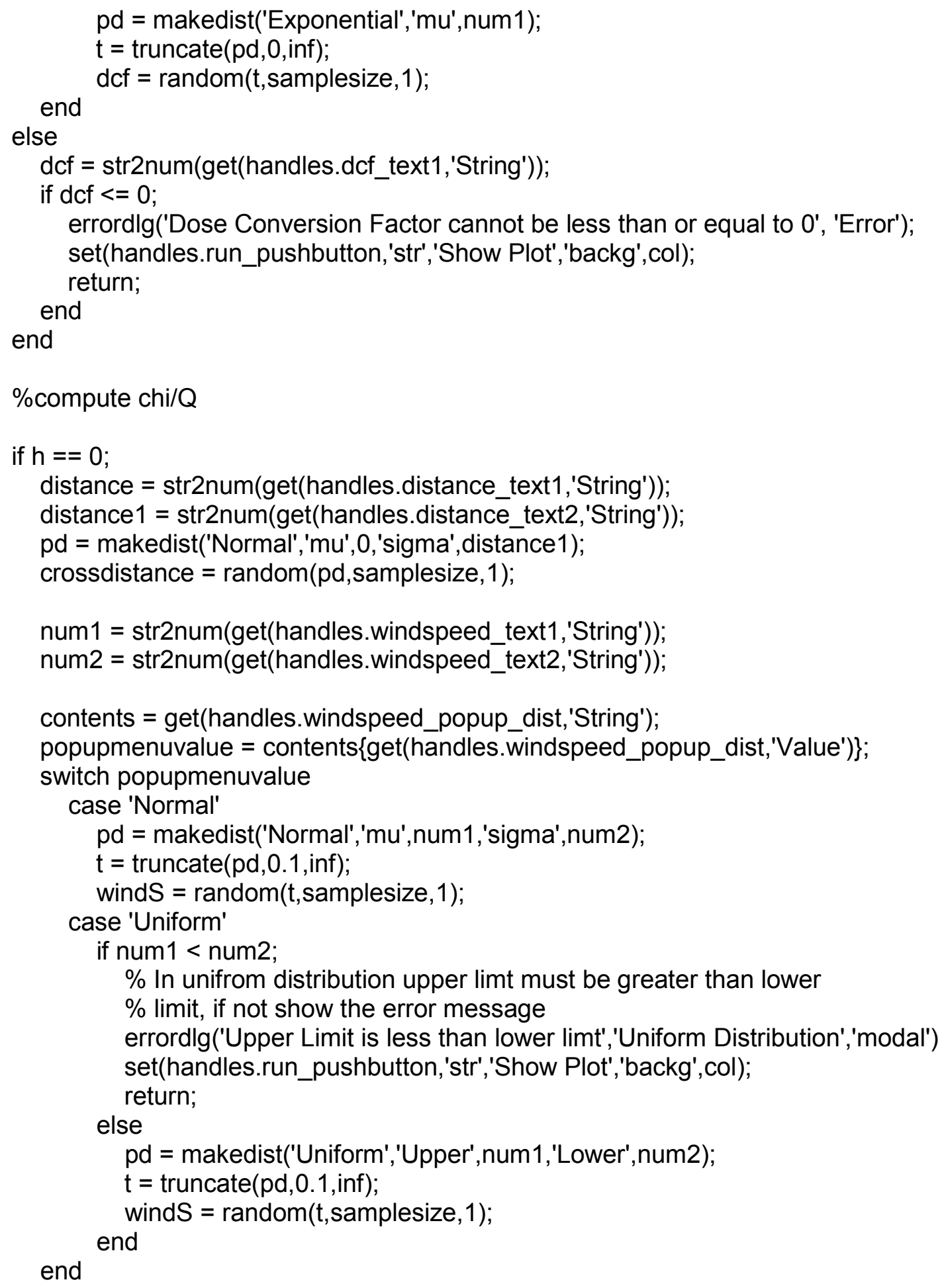


stability = contents3\{get(handles.stability_popup,'Value')\};

height = str2num(get(handles.height_text,'String'));

switch terrainvalue

case 'Rural/Open Country'

switch stability

case 'A'

sigma_y $=0.22^{*}{ }^{*}$ istance ${ }^{*}\left(1+0.0001^{*} \text { distance }\right)^{\wedge}(-0.5)$;

sigma_z $=0.20^{*}$ distance;

case 'B'

sigma_y $=0.16^{*}$ distance ${ }^{*}\left(1+0.0001^{*} \text { distance }\right)^{\wedge}(-0.5)$;

sigma_z $=0.12^{*}$ distance;

case ' $\mathrm{C}$ '

sigma_y $=0.11^{*} \operatorname{distance}^{*}\left(1+0.0001^{*} \text { distance }\right)^{\wedge}(-0.5)$;

sigma_z $=0.08^{*}$ distance $^{*}\left(1+0.0002^{*} \text { distance }\right)^{\wedge}(-0.5)$;

case 'D'

sigma_y $=0.08^{*}$ distance ${ }^{*}\left(1+0.0001^{*} \text { distance }\right)^{\wedge}(-0.5)$;

sigma_z $=0.06^{*} \operatorname{distance}^{*}\left(1+0.0015^{*} \text { distance }\right)^{\wedge}(-0.5)$;

case 'E'

sigma_y $=0.06^{*}$ distance $^{*}\left(1+0.0001^{*} \text { distance }\right)^{\wedge}(-0.5)$;

sigma_z $=0.03^{*} \operatorname{distance}^{*}\left(1+0.0003^{*} \text { distance }\right)^{\wedge}(-1)$;

case ' $F$ '

sigma_y $=0.04^{*}$ distance ${ }^{*}\left(1+0.0001^{*} \text { distance }\right)^{\wedge}(-0.5)$;

sigma_z $=0.016^{*}$ distance ${ }^{*}\left(1+0.0003^{*} \text { distance }\right)^{\wedge}(-1)$;

case 'Select Stability Condition'

errordlg('Select Stability Conditions','Error','modal');

set(handles.run_pushbutton,'str','Show Plot','backg',col);

return;

end

case 'Select Terrain'

switch stability

case 'A'

errordlg('Select terrain','Error','modal');

set(handles.run_pushbutton,'str','Show Plot','backg',col);

return;

case 'B'

errordlg('Select terrain','Error','modal')

set(handles.run_pushbutton,'str','Show Plot','backg',col);

return;

case ' $C$ '

errordlg('Select terrain','Error','modal');

set(handles.run_pushbutton,'str','Show Plot','backg',col);

return;

case 'D'

errordlg('Select terrain','Error','modal');

set(handles.run_pushbutton,'str','Show Plot','backg',col);

return;

case 'E' 


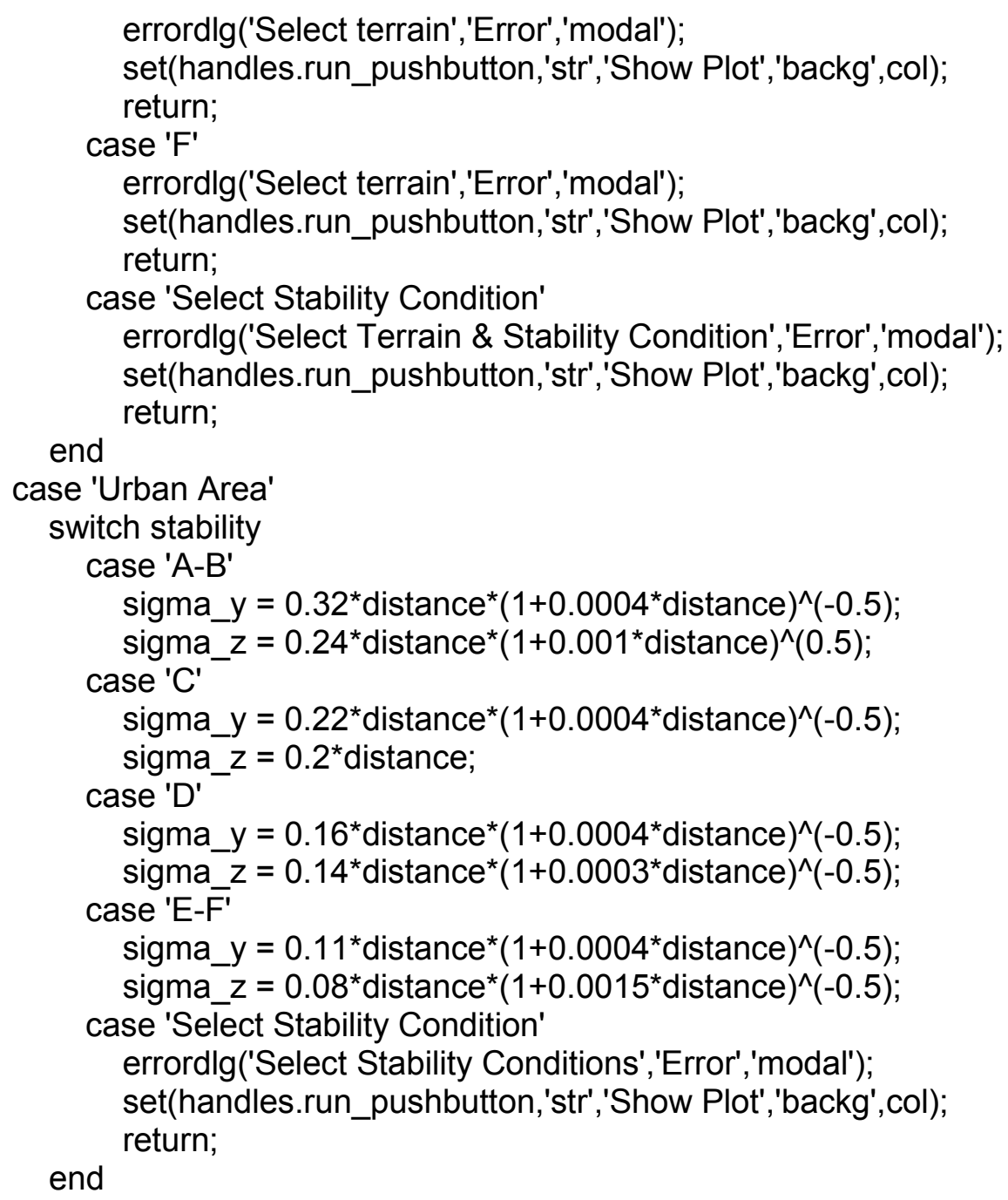




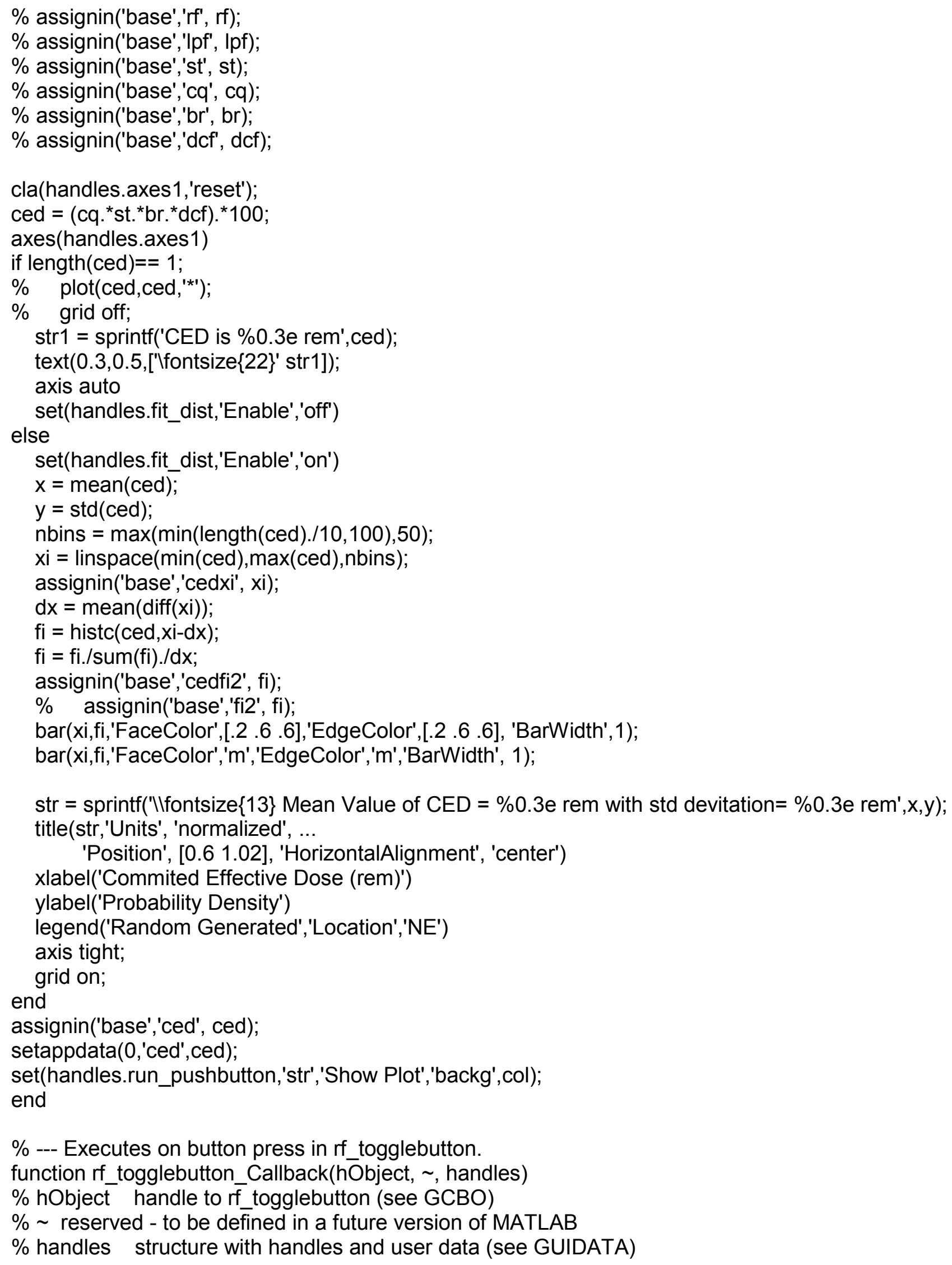


\% Hint: get(hObject,'Value') returns toggle state of rf_togglebutton ispushed = get(hObject,'Value');

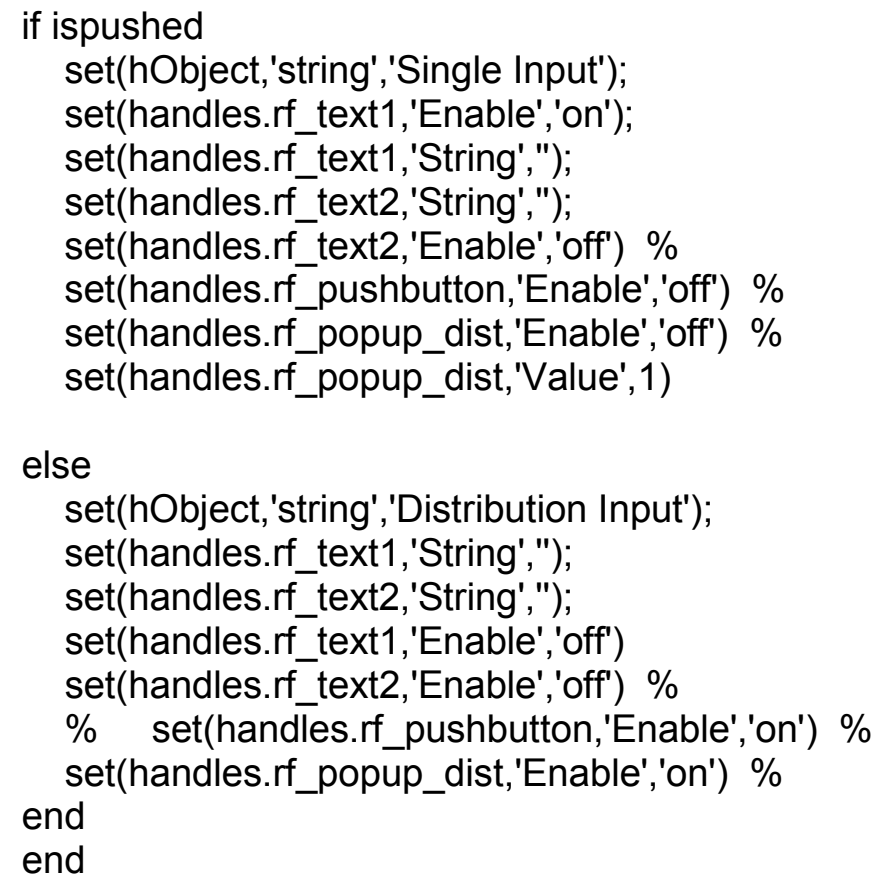


end

end

$\%$--- Executes on button press in cq_pushbutton.

function cq_pushbutton_Callback(hObject, , handles)

$\%$ hObject handle to cq_pushbutton (see GCBO)

$\% \sim$ reserved - to be defined in a future version of MATLAB

$\%$ handles structure with handles and user data (see GUIDATA)

sample = get(handles.num_sample_text,'String');

samplesize $=$ str2num(sample);

if strcmp(sample,") == 1 || samplesize $<0$

errordlg('Please enter number of samples','Sample Number','modal');

return;

end

col = get(handles.cq_pushbutton,'backg');

set(handles.cq_pushbutton,'str','RUNNING...','backg',[.2 .6 .6]);

distance $=\operatorname{str} 2$ num $(g e t($ handles.distance_text 1 ,'String'));

distance1 = str2num(get(handles.distance_text2,'String'));

pd = makedist('Normal','mu',0,'sigma',distance1);

crossdistance $=$ random $(p d$, samplesize, 1$)$;

num1 = str2num(get(handles.windspeed_text1,'String'));

num2 = str2num(get(handles.windspeed_text2,'String'));

contents = get(handles.windspeed_popup_dist,'String');

popupmenuvalue = contents $\{$ get(handles.windspeed_popup_dist,'Value')\};

switch popupmenuvalue

case 'Normal'

pd = makedist('Normal','mu',num1,'sigma',num2);

$\mathrm{t}=$ truncate $(\mathrm{pd}, 0.1$,inf $)$;

wind $\mathrm{S}=$ random $(\mathrm{t}$, samplesize, 1$)$;

case 'Uniform'

if num1 < num2;

$\%$ In unifrom distribution upper limt must be greater than lower

$\%$ limit, if not show the error message

errordlg('Upper Limit is less than lower limt','Uniform Distribution')

set(handles.cq_pushbutton,'str','Show Plot','backg',col);

return;

else

pd = makedist('Uniform','Upper',num1,'Lower',num2);

$\mathrm{t}=$ truncate $(\mathrm{pd}, 0.1$,inf);

windS = random(t,samplesize, 1$)$;

end

end

contents2 = get(handles.terrain_popup,'String');

terrainvalue $=$ contents $2\{$ get $($ handles.terrain_popup,'Value') $\}$; 
contents3 = get(handles.stability_popup,'String');

stability = contents $3\{$ get(handles.stability_popup,'Value')\};

height = str2num(get(handles.height_text,'String'));

switch terrainvalue

case 'Rural/Open Country'

switch stability

case 'A'

sigma_y $=0.22^{*}$ distance ${ }^{*}\left(1+0.0001^{*} \text { distance }\right)^{\wedge}(-0.5)$;

sigma_z $=0.20^{*}$ distance;

case 'B'

sigma_y $=0.16^{*}$ distance ${ }^{*}\left(1+0.0001^{*} \text { distance }\right)^{\wedge}(-0.5)$;

sigma_z $=0.12^{*}$ distance;

case ' $\mathrm{C}$ '

sigma_y $=0.11^{*}$ distance $^{*}\left(1+0.0001^{*} \text { distance }\right)^{\wedge}(-0.5)$;

sigma_z $=0.08^{*} \operatorname{distance}^{*}\left(1+0.0002^{*} \text { distance }\right)^{\wedge}(-0.5)$;

case 'D'

sigma_y $=0.08^{*}$ distance $^{*}\left(1+0.0001^{*} \text { distance }\right)^{\wedge}(-0.5)$;

sigma_z $=0.06^{*}$ distance $^{*}\left(1+0.0015^{*} \text { distance }\right)^{\wedge}(-0.5)$;

case 'E'

sigma_y $=0.06^{*}$ distance ${ }^{*}\left(1+0.0001^{*} \text { distance }\right)^{\wedge}(-0.5)$;

sigma_z $=0.03^{*} \operatorname{distance}^{*}\left(1+0.0003^{*} \text { distance }\right)^{\wedge}(-1)$;

case ' $F$ '

sigma_y $=0.04^{*}$ distance ${ }^{*}\left(1+0.0001^{*} \text { distance }\right)^{\wedge}(-0.5)$;

sigma_z $=0.016^{*}$ distance ${ }^{*}\left(1+0.0003^{*} \text { distance }\right)^{\wedge}(-1)$;

case 'Select Stability Condition'

errordlg('Select Stability Conditions','Error','modal');

set(handles.cq_pushbutton,'str','Show Plot','backg',col);

return;

end

case 'Select Terrain'

switch stability

case 'A'

errordlg('Select terrain','Error','modal');

set(handles.cq_pushbutton,'str','Show Plot','backg',col);

return;

case 'B'

errordlg('Select terrain"Error','modal')

set(handles.cq_pushbutton,'str','Show Plot','backg',col);

return;

case ' $\mathrm{C}$ '

errordlg('Select terrain"Error','modal');

set(handles.cq_pushbutton,'str','Show Plot','backg',col);

return;

case ' $D$ '

errordlg('Select terrain"Error','modal');

set(handles.cq_pushbutton,'str','Show Plot','backg',col); 


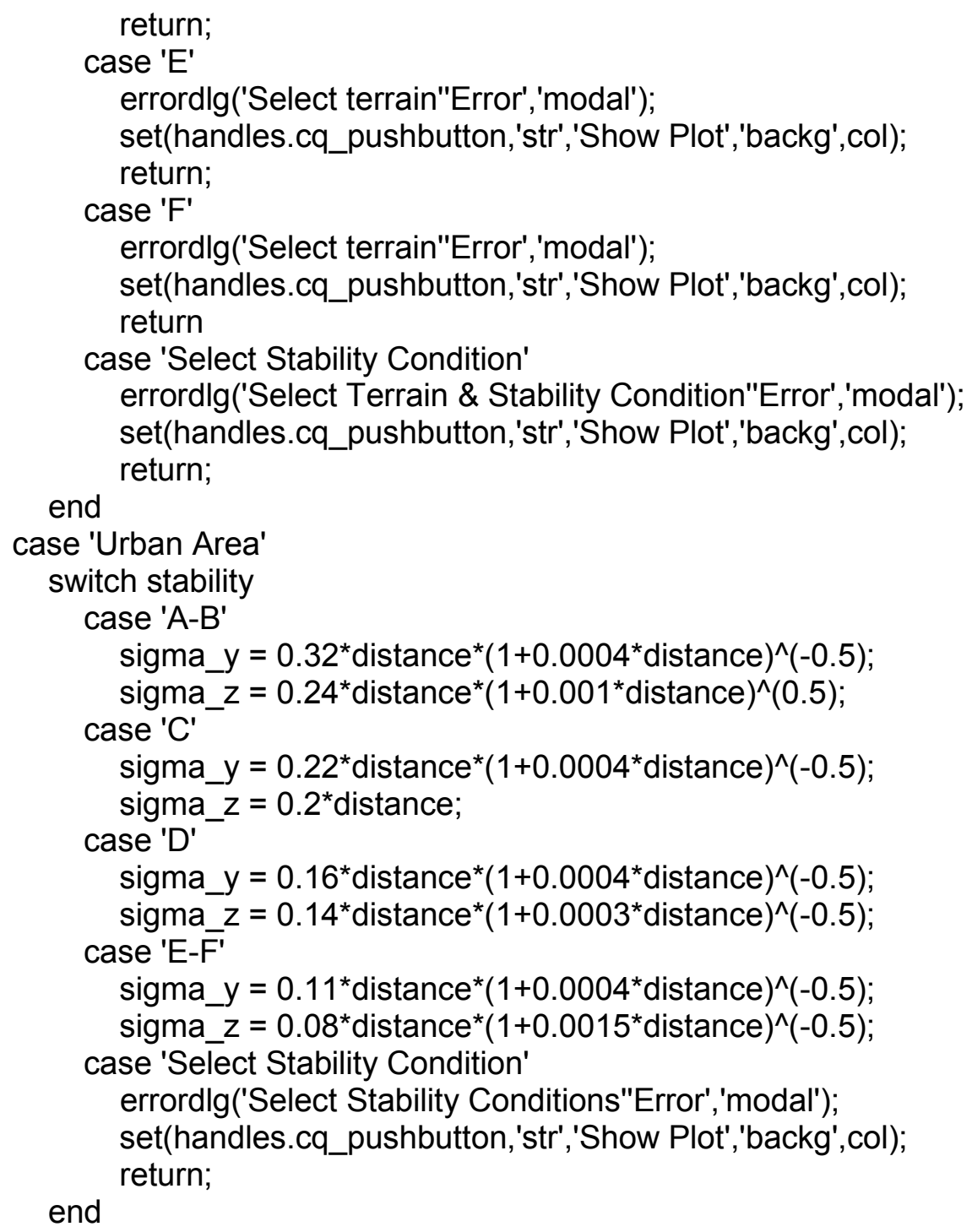


$\% \operatorname{hist}(\mathrm{c}, 50)$

xlabel('Chi/Q')

ylabel('Probability Density')

title([ 'Ichi/Q' ' Distribution with Imu= ' num2str(mean(c)) ' and Isigma= ' num2str(std(c))])

set(handles.cq_pushbutton,'str','Show Plot','backg',col);

assignin('base','cq', c);

end

function cq_text1_Callback(hObject, , handles)

$\%$ hObject handle to cq_text1 (see GCBO)

$\% \sim$ reserved - to be defined in a future version of MATLAB

$\%$ handles structure with handles and user data (see GUIDATA)

end

$\%$ Hints: get(hObject,'String') returns contents of cq_text1 as text

$\% \quad$ str2double(get(hObject,'String')) returns contents of cq_text1 as a double

$\%$--- Executes during object creation, after setting all properties.

function cq_text1_CreateFcn(hObject, , handles)

$\%$ hObject handle to cq_text1 (see GCBO)

$\% \sim$ reserved - to be defined in a future version of MATLAB

$\%$ handles empty - handles not created until after all CreateFcns called

$\%$ Hint: edit controls usually have a white background on Windows.

$\% \quad$ See ISPC and COMPUTER.

if ispc \&\& isequal(get(hObject,'BackgroundColor'), get(0,'defaultUicontrolBackgroundColor')) set(hObject,'BackgroundColor','white');

end

end

$\%$--- Executes on selection change in cq_popup_dist.

function cq_popup_dist_Callback(hObject, , handles)

$\%$ hObject handle to cq_popup_dist (see GCBO)

$\%$ reserved - to be defined in a future version of MATLAB

$\%$ handles structure with handles and user data (see GUIDATA)

contents = cellstr(get $($ hObject,'String'));

cqpopchoice $=$ contents $\left\{\right.$ get $\left(\right.$ hObject, ${ }^{\prime}$ Value' $\left.)\right\}$;

switch cqpopchoice

case 'Normal'

set(handles.cq_text1,'Enable','inactive') \%

set(handles.cq_text2,'Enable','inactive') \%

set(handles.cq_pushbutton,'Enable','on') \%

set(handles.cq_text1,'String','Mean');

set(handles.cq_text2,'String','Std Deviation'); 


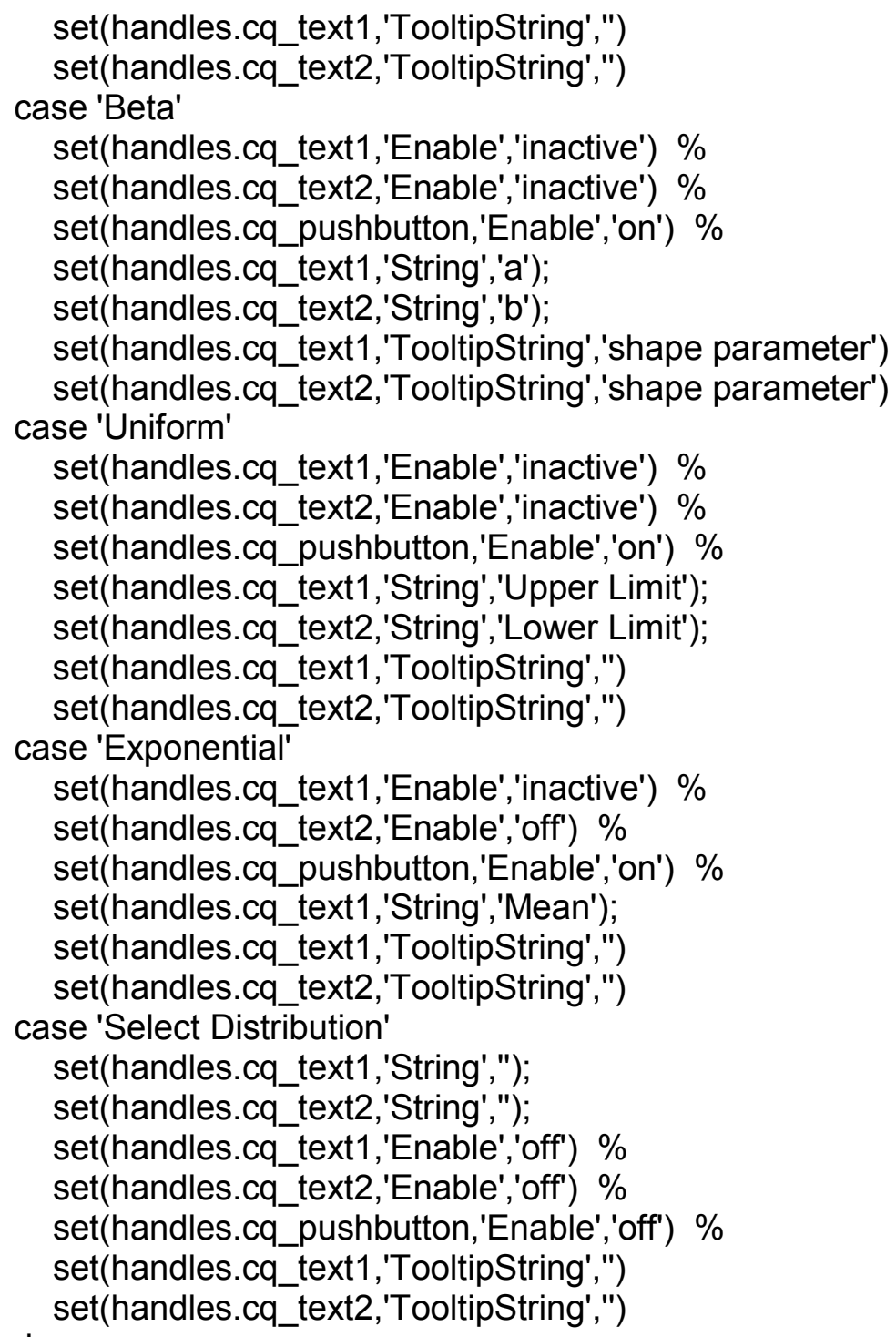

$\%$--- Executes during object creation, after setting all properties.

function cq_popup_dist_CreateFcn(hObject, , handles)

$\%$ hObject handle to cq_popup_dist (see GCBO)

$\% \sim$ reserved - to be defined in a future version of MATLAB

$\%$ handles empty - handles not created until after all CreateFcns called

$\%$ Hint: popupmenu controls usually have a white background on Windows.

$\% \quad$ See ISPC and COMPUTER.

if ispc \&\& isequal(get(hObject,'BackgroundColor'), get( 0 ,'defaultUicontrolBackgroundColor')) set(hObject,'BackgroundColor','white');

end

end 
$\%$--- Executes on button press in cq_togglebutton.

function cq_togglebutton_Callback(hObject, , handles)

$\%$ hObject handle to cq_togglebutton (see GCBO)

$\% \sim$ reserved - to be defined in a future version of MATLAB

$\%$ handles structure with handles and user data (see GUIDATA)

ispushed = get(hObject,'Value');

if ispushed

set(hObject,'string','Single Input');

set(handles.cq_text1,'Enable','on');

set(handles.cq_text1,'String',");

set(handles.windspeed_text1,'String',");

set(handles.windspeed_text2,'String',");

set(handles.windspeed_text1,'Enable','off');

set(handles.windspeed_text2,'Enable','off');

set(handles.height_text,'String',");

set(handles.height_text,'Enable','off') ; \%

set(handles.cq_pushbutton,'Enable','off'); \%

set(handles.terrain_popup,'Enable','off'); \%

set(handles.terrain_popup,'Value',1)

set(handles.stability_popup,'Enable','off'); \%

set(handles.stability_popup,'Value',1)

set(handles.windspeed_popup_dist,'Enable','off'); \%

set(handles.windspeed_popup_dist,'Value',1);

set(handles.distance_text1,'String',");

set(handles.distance_text1,'Enable','off') ; \%

set(handles.distance_text2,'String',");

set(handles.distance_text2,'Enable','off') ; \%

else

set(hObject,'string','Distribution Input');

set(handles.cq_text1,'Enable','off');

set(handles.cq_text1,'String',");

set(handles.windspeed_text1,'String',");

set(handles.windspeed_text1,'Enable','on') ;

set(handles.windspeed_text2,'String',");

set(handles.windspeed_text2,'Enable','on') ; \%

set(handles.height_text,'String','Height');

set(handles.height_text,'Enable','on') ; \%

set(handles.cq_pushbutton,'Enable','off'); \%

set(handles.terrain_popup,'Enable','on'); \%

set(handles.terrain_popup,'Value',1)

set(handles.stability_popup,'Enable','off'); \%

set(handles.stability_popup,' 'Value',1)

set(handles.distance_text1,'String',");

set(handles.distance_text1,'Enable','on') ; \%

set(handles.distance_text2,'String',");

set(handles.distance_text2,'Enable','on') ; \%

set(handles.windspeed_popup_dist,'Enable','on'); \%

set(handles.windspeed_popup_dist,'Value',1)

set(handles.windspeed_text1,'String',"); 


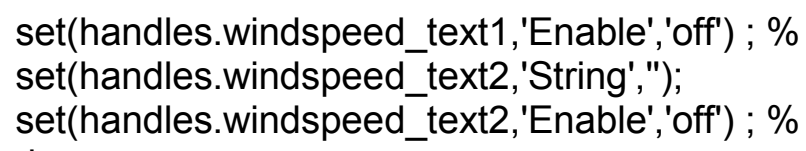

\% Hint: get(hObject,'Value') returns toggle state of cq_togglebutton

$\%$--- Executes on selection change in dcf_popup_dist.

function dcf_popup_dist_Callback(hObject, , handles)

$\%$ hObject handle to dcf_popup_dist (see GCBO)

$\% \sim$ reserved - to be defined in a future version of MATLAB

$\%$ handles structure with handles and user data (see GUIDATA)

contents = cellstr(get(hObject,'String'));

dcfpopchoice $=$ contents $\{$ get $($ hObject,'Value') $\}$;

switch dcfpopchoice

case 'Normal' set(handles.dcf_text1,'Enable','inactive') \%

set(handles.dcf_text2,'Enable','inactive') \%

set(handles.dcf_pushbutton,'Enable','on') \%

set(handles.dcf_text1,'String','Mean');

set(handles.dcf_text2,'String','Std Deviation');

case 'Beta'

set(handles.dcf_text1,'Enable','inactive') \%

set(handles.dcf text2,'Enable','inactive') \%

set(handles.dcf_pushbutton,'Enable','on') \%

set(handles.dcf_text1,'String','a');

set(handles.dcf_text2,'String','b');

set(handles.dcf_text1,'TooltipString','shape parameter')

set(handles.dcf_text2,'TooltipString','shape parameter')

case 'Uniform'

set(handles.dcf_text1,'Enable','inactive') \%

set(handles.dcf_text2,'Enable','inactive') \%

set(handles.dcf_pushbutton,'Enable','on') \%

set(handles.dcf_text1,'String','Upper Limit');

set(handles.dcf_text2,'String','Lower Limit');

case 'Exponential'

set(handles.dcf_text1,'Enable','inactive') \%

set(handles.dcf_text2,'Enable','off') \%

set(handles.dcf_pushbutton,'Enable','on') \%

set(handles.dcf_text1,'String','Mean');

set(handles.dcf_text2,'String',");

case 'Select Distribution'

set(handles.dcf_text1,'String',");

set(handles.dcf_text2,'String',");

set(handles.dcf_text1,'Enable','off') \%

set(handles.dcf_text2,'Enable','off') \%

set(handles.dcf_pushbutton,'Enable','off') \%

case 'U-238'

set(handles.dcf_text1,'String','5.0*10^-7');

case 'Select Isotope' 


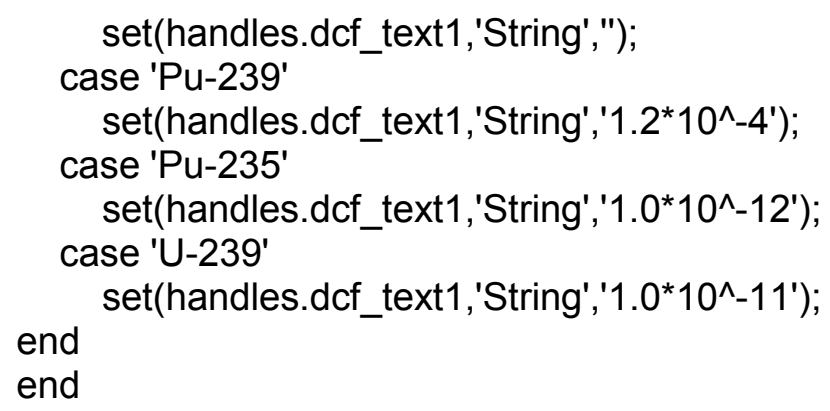

$\%$ Hints: contents = cellstr(get(hObject,'String')) returns dcf_popup_dist contents as cell array

$\% \quad$ contents $\{$ get(hObject,'Value')\} returns selected item from dcf_popup_dist

$\%$--- Executes during object creation, after setting all properties.

function dcf_popup_dist_CreateFcn(hObject, , handles)

$\%$ hObject handle to dcf_popup_dist (see GCBO)

$\% \sim$ reserved - to be defined in a future version of MATLAB

$\%$ handles empty - handles not created until after all CreateFcns called

$\%$ Hint: popupmenu controls usually have a white background on Windows.

$\% \quad$ See ISPC and COMPUTER.

if ispc \&\& isequal(get(hObject,'BackgroundColor'), get(0,'defaultUicontrolBackgroundColor')) set(hObject,'BackgroundColor','white');

end

end

function dcf_text1_Callback(hObject, , handles)

$\%$ hObject handle to dcf_text1 (see GCBO)

$\% \sim$ reserved - to be defined in a future version of MATLAB

$\%$ handles structure with handles and user data (see GUIDATA)

end

$\%$ Hints: get(hObject,'String') returns contents of dcf_text1 as text

$\% \quad$ str2double(get(hObject,'String')) returns contents of dcf_text1 as a double

$\%$--- Executes during object creation, after setting all properties.

function dcf_text1_CreateFcn(hObject, , handles)

$\%$ hObject handle to dcf_text1 (see GCBO)

$\% \sim$ reserved - to be defined in a future version of MATLAB

$\%$ handles empty - handles not created until after all CreateFcns called

$\%$ Hint: edit controls usually have a white background on Windows.

$\% \quad$ See ISPC and COMPUTER.

if ispc \&\& isequal(get(hObject,'BackgroundColor'), get(0,'defaultUicontrolBackgroundColor')) set(hObject,'BackgroundColor','white');

end

end

function dcf_text2_Callback(hObject, , handles) 
$\%$ hObject handle to dcf_text2 (see GCBO)

$\%$ reserved - to be defined in a future version of MATLAB

$\%$ handles structure with handles and user data (see GUIDATA)

end

\% Hints: get(hObject,'String') returns contents of dcf_text2 as text

$\% \quad$ str2double(get(hObject,'String')) returns contents of dcf_text2 as a double

$\%$--- Executes during object creation, after setting all properties.

function dcf_text2_CreateFcn(hObject, , handles)

$\%$ hObject handle to dcf_text2 (see GCBO)

$\% \sim$ reserved - to be defined in a future version of MATLAB

$\%$ handles empty - handles not created until after all CreateFcns called

$\%$ Hint: edit controls usually have a white background on Windows.

$\% \quad$ See ISPC and COMPUTER.

if ispc \&\& isequal(get(hObject,'BackgroundColor'), get(0,'defaultUicontrolBackgroundColor')) set(hObject,'BackgroundColor','white');

end

end

$\%$--- Executes on button press in dcf_pushbutton.

function dcf_pushbutton_Callback(hObject, , handles)

$\%$ hObject handle to dcf_pushbutton (see GCBO)

$\% \sim$ reserved - to be defined in a future version of MATLAB

$\%$ handles structure with handles and user data (see GUIDATA)

sample = get(handles.num_sample_text,'String');

samplesize $=$ str2num(sample);

if strcmp(sample,") == 1 || samplesize $<0$

errordlg('Please enter number of samples','Sample Number','modal');

return;

end

col = get(handles.dcf_pushbutton,'backg');

set(handles.dcf_pushbutton,'str','RUNNING...','backg',[.2 .6 .6]);

pause(eps);

num1 = str2num(get(handles.dcf_text1,'String'));

num2 = str2num(get(handles.dcf_text2,'String'));

contents = get(handles.dcf_popup_dist,'String');

popupmenuvalue = contents $\{$ get(handles.dcf_popup_dist,'Value')\};

cla(handles.axes1,'reset');

switch popupmenuvalue

case 'Normal'

pd = makedist('Normal','mu',num1,'sigma',num2);

$\mathrm{t}=$ truncate(pd,0,inf);

$\mathrm{n}=$ random(t,samplesize, 1$)$;

axes(handles.axes1)

nbins $=\max (\min ($ length $(n) . / 10,100), 50)$;

$x i=$ linspace $(\min (n), \max (n)$, nbins $)$;

$\mathrm{dx}=\operatorname{mean}(\operatorname{diff}(\mathrm{xi}))$;

fi $=\operatorname{histc}(n, x i-d x)$;

$\mathrm{fi}=\mathrm{fi} . / \operatorname{sum}(\mathrm{fi}) . / \mathrm{dx}$; 


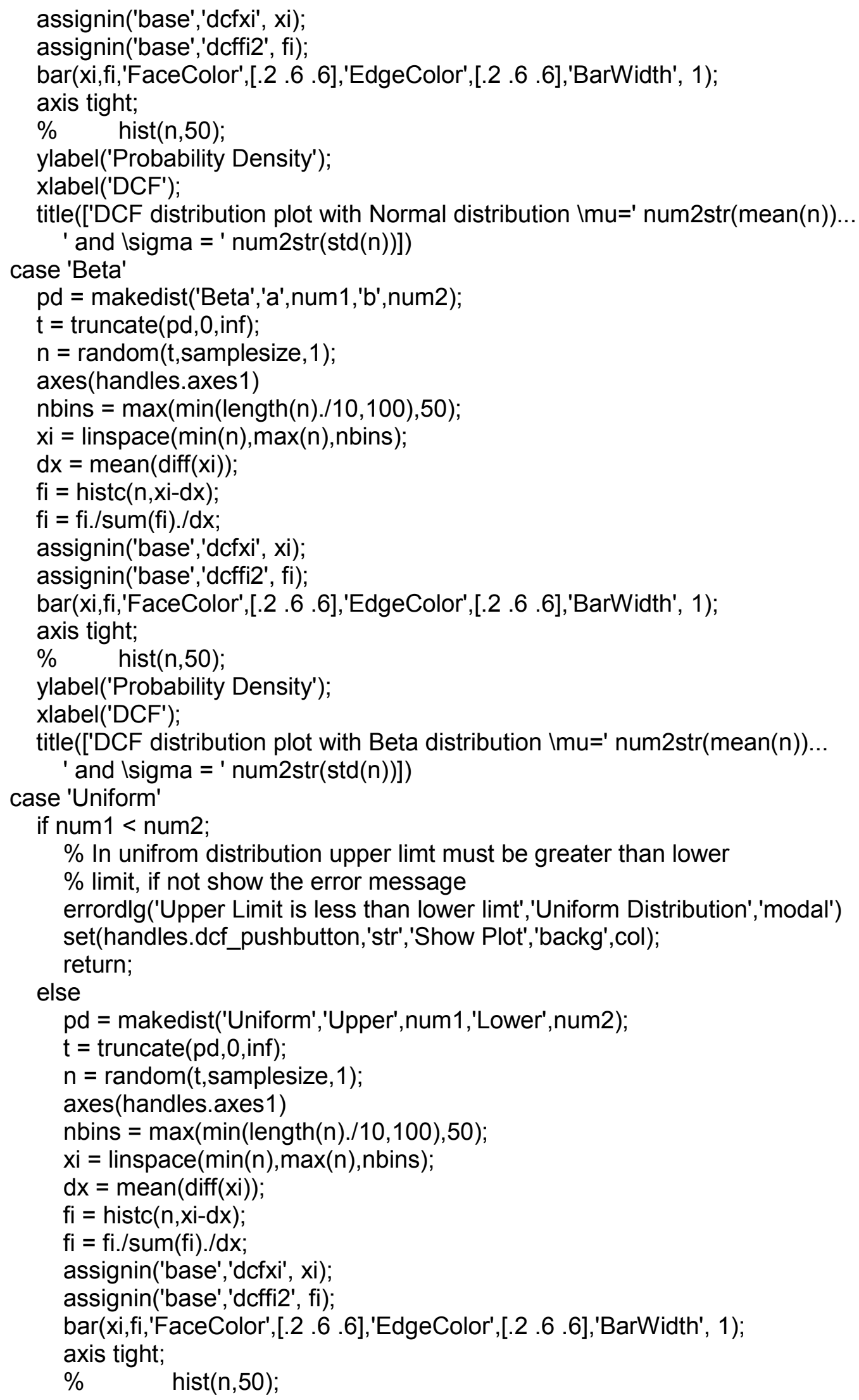




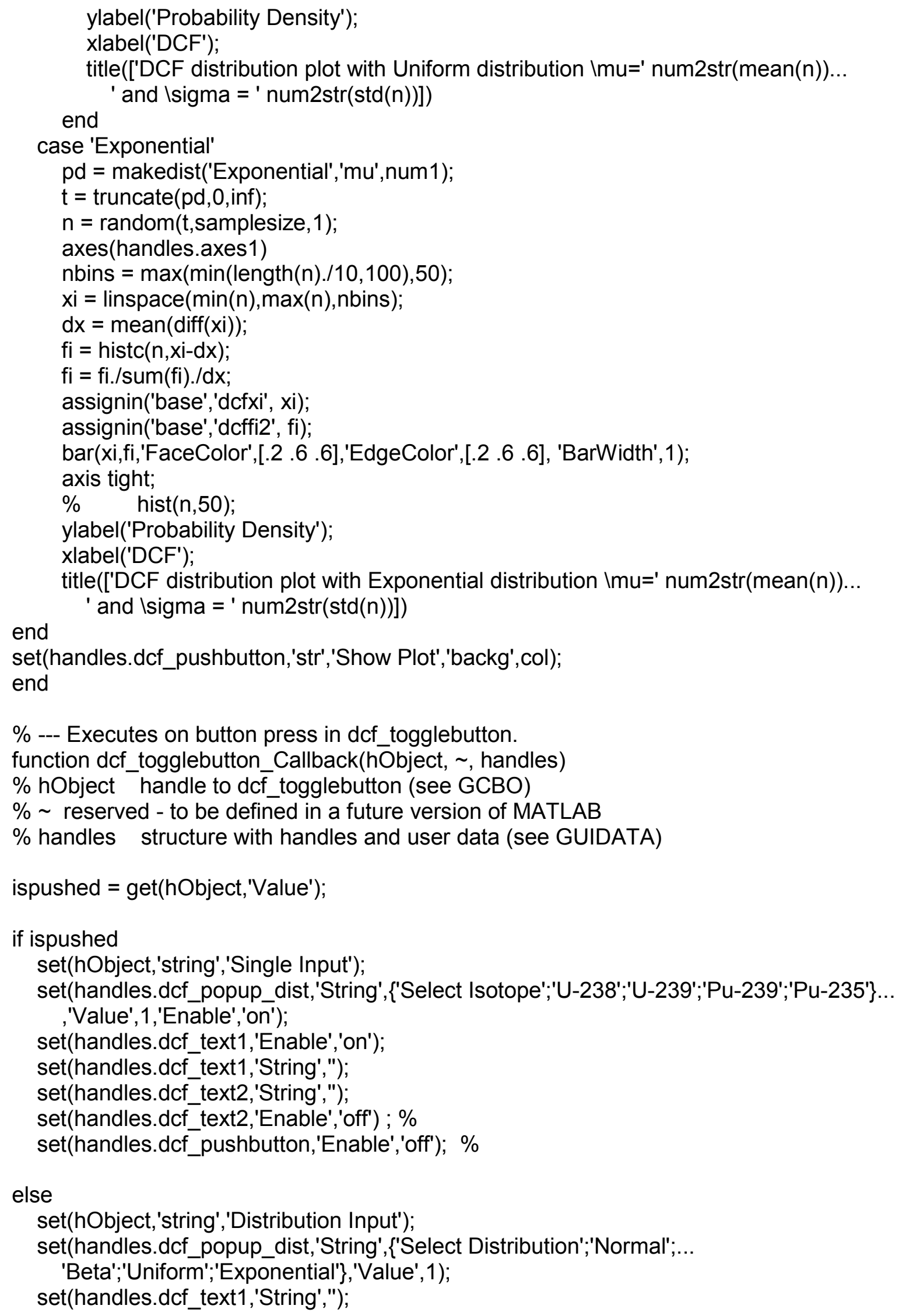




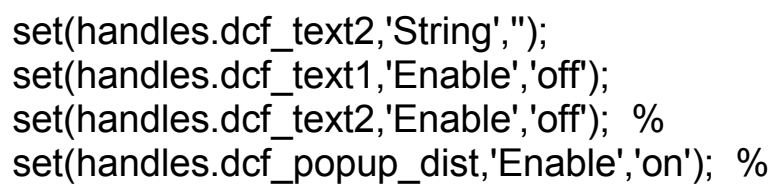

end

end

\% Hint: get(hObject,'Value') returns toggle state of dcf_togglebutton

$\%$--- Executes on button press in br_pushbutton.

function br_pushbutton_Callback(hObject, , handles)

$\%$ hObject handle to br_pushbutton (see GCBO)

$\%$ reserved - to be defined in a future version of MATLAB

$\%$ handles structure with handles and user data (see GUIDATA)

sample = get(handles.num_sample_text,'String');

samplesize $=$ str2num(sample);

if strcmp(sample,") == 1 || samplesize $<0$

errordlg('Please enter number of samples','Sample Number','modal');

return;

end

col = get(handles.br_pushbutton,'backg');

set(handles.br_pushbutton,'str','RUNNING...','backg',[.2 .6 .6]);

pause(eps);

$a=8.33^{*} 10^{\wedge}-4$;

$\mathrm{b}=4.17^{*} 10^{\wedge}-4$

$c=1.5^{*} 10^{\wedge}-4$

$d=1.25^{*} 10^{\wedge}-4$

for e = 1:samplesize;

num_rand=rand;

if num_rand $<=0.17$

$\mathrm{n}(\mathrm{e})=\operatorname{rand}^{*}(\mathrm{a}-\mathrm{b})+\mathrm{b}$;

elseif num_rand $>0.17 \& \&$ num_rand $<=0.34$;

$\mathrm{n}(\mathrm{e})=\operatorname{rand}^{*}(\mathrm{~b}-\mathrm{c})+\mathrm{c}$;

elseif num_rand $>0.34$

end

$n(e)=\operatorname{rand}^{*}(c-d)+d$;

end

$\mathrm{n}=\mathrm{n}$;

cla(handles.axes1,'reset');

axes(handles.axes1);

nbins $=\max (\min ($ length $(\mathrm{n}) \cdot / 10,100), 50)$;

$x i=$ linspace $(\min (n), \max (n), n b i n s)$;

$\mathrm{dx}=\operatorname{mean}(\mathrm{diff}(\mathrm{xi}))$;

$\mathrm{fi}=\operatorname{histc}(\mathrm{n}, \mathrm{xi}-\mathrm{dx})$;

$\mathrm{fi}=\mathrm{fi} . / \mathrm{sum}(\mathrm{fi}) . / \mathrm{dx}$;

assignin('base','brxi', xi);

assignin('base','brfi2', fi);

bar(xi,fi,''FaceColor',[.2 .6 .6],'EdgeColor',[.2 .6 .6], 'BarWidth',1);

axis tight; 


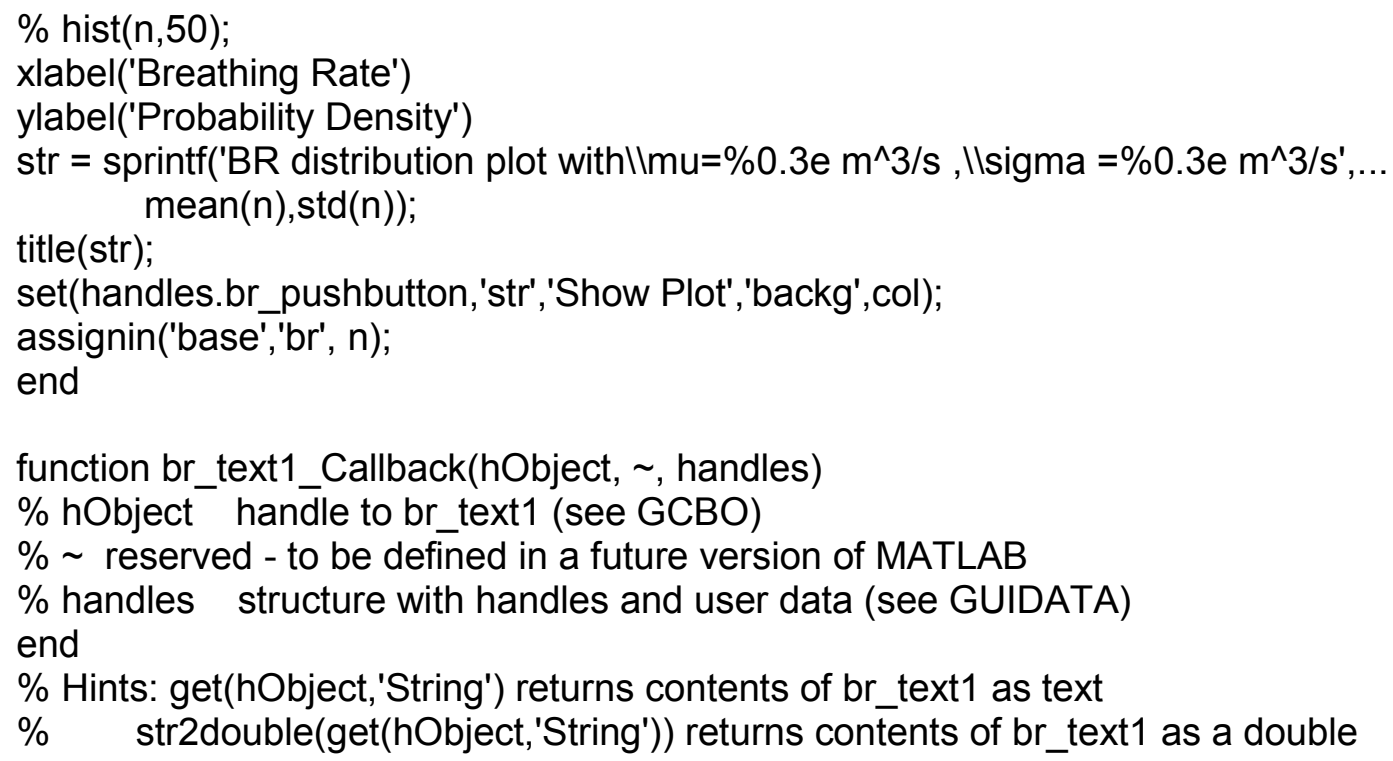

$\%$--- Executes during object creation, after setting all properties.

function br_text1_CreateFcn(hObject, , handles)

$\%$ hObject handle to br_text1 (see GCBO)

$\% \sim$ reserved - to be defined in a future version of MATLAB

$\%$ handles empty - handles not created until after all CreateFcns called

$\%$ Hint: edit controls usually have a white background on Windows.

$\% \quad$ See ISPC and COMPUTER.

if ispc \&\& isequal(get(hObject,'BackgroundColor'), get( 0 ,'defaultUicontrolBackgroundColor')) set(hObject,'BackgroundColor','white');

end

end

$\%$--- Executes on button press in br_togglebutton.

function br_togglebutton_Callback(hŌbject, , handles)

$\%$ hObject handle to br_togglebutton (see GCBO)

$\% \sim$ reserved - to be defined in a future version of MATLAB

$\%$ handles structure with handles and user data (see GUIDATA)

ispushed = get(hObject,'Value');

if ispushed

set(hObject,'string','Single Input');

set(handles.br_text1,'Enable','on');

set(handles.br_text1,'String',");

set(handles.br_pushbutton,'Enable','off'); \%

else

set(hObject,'string','Distribution Input');

set(handles.br_text1,'Enable','off','String',");

set(handles.br_pushbutton,'Enable','on');

end

end 
\% Hint: get(hObject,'Value') returns toggle state of br_togglebutton

$\%$--- Executes on selection change in Ipf_popup_dist.

function Ipf_popup_dist_Callback(hObject, , handles)

$\%$ hObject handle to Ipf_popup_dist (see GCBO)

$\% \sim$ reserved - to be defined in a future version of MATLAB

$\%$ handles structure with handles and user data (see GUIDATA)

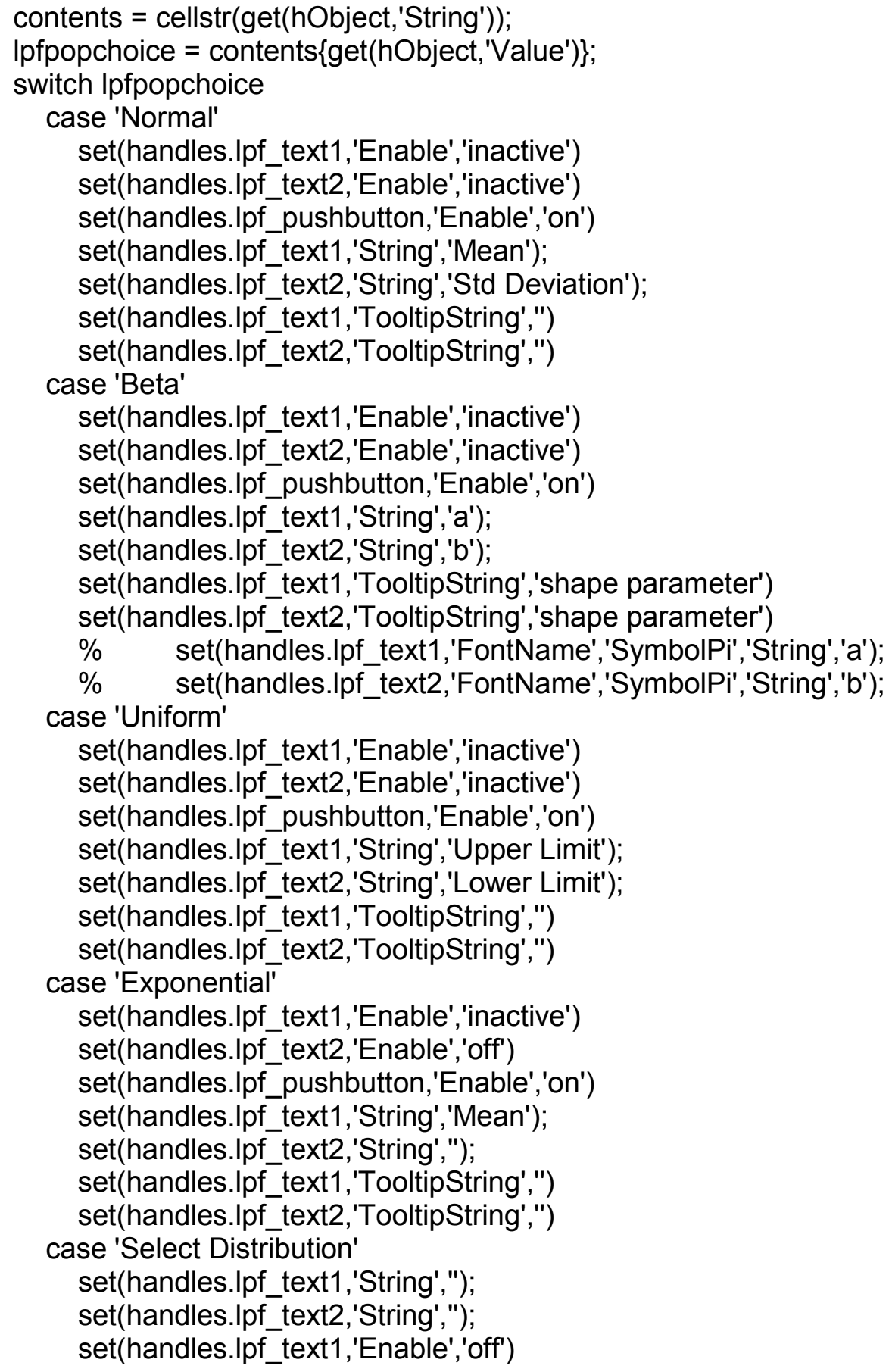




$$
\begin{aligned}
& \text { set(handles.Ipf_text2,'Enable','off') } \\
& \text { set(handles.Ipf_pushbutton,'Enable','off') } \\
& \text { set(handles.Ipf_text1,'TooltipString',") } \\
& \text { set(handles.Ipf_text2,'TooltipString',"') }
\end{aligned}
$$

end

end

$\%$ Hints: contents = cellstr(get(hObject,'String')) returns Ipf_popup_dist contents as cell array

$\% \quad$ contents\{get(hObject,'Value')\} returns selected item from Ipf_popup_dist

$\%$--- Executes during object creation, after setting all properties.

function Ipf_popup_dist_CreateFcn(hObject, , handles)

$\%$ hObject handle to Ipf popup dist (see GCBO)

$\% \sim$ reserved - to be defined in a future version of MATLAB

$\%$ handles empty - handles not created until after all CreateFcns called

$\%$ Hint: popupmenu controls usually have a white background on Windows.

$\% \quad$ See ISPC and COMPUTER.

if ispc \&\& isequal(get(hObject,'BackgroundColor'), get(0,'defaultUicontrolBackgroundColor')) set(hObject,'BackgroundColor','white');

end

end

function Ipf_text1_Callback(hObject, , handles)

$\%$ hObject handle to Ipf_text1 (see GCBO)

$\% \sim$ reserved - to be defined in a future version of MATLAB

$\%$ handles structure with handles and user data (see GUIDATA)

end

\% Hints: get(hObject,'String') returns contents of Ipf_text1 as text

$\% \quad$ str2double(get(hObject,'String')) returns contents of Ipf_text1 as a double

$\%$--- Executes during object creation, after setting all properties.

function Ipf_text1_CreateFcn(hObject, , handles)

$\%$ hObject handle to Ipf_text1 (see GCBO)

$\% \sim$ reserved - to be defined in a future version of MATLAB

$\%$ handles empty - handles not created until after all CreateFcns called

$\%$ Hint: edit controls usually have a white background on Windows.

$\% \quad$ See ISPC and COMPUTER.

if ispc \&\& isequal(get(hObject,'BackgroundColor'), get(0,'defaultUicontrolBackgroundColor')) set(hObject,'BackgroundColor','white');

end

end

function Ipf_text2_Callback(hObject, , handles)

$\%$ hObject handle to Ipf_text2 (see GCBO)

$\% \sim$ reserved - to be defined in a future version of MATLAB

$\%$ handles structure with handles and user data (see GUIDATA) 
end

$\%$ Hints: get(hObject,'String') returns contents of Ipf_text2 as text

$\% \quad$ str2double(get(hObject,'String')) returns contents of Ipf_text2 as a double

$\%$--- Executes during object creation, after setting all properties.

function Ipf_text2_CreateFcn(hObject, , handles)

$\%$ hObject handle to Ipf_text2 (see GCBO)

$\% \sim$ reserved - to be defined in a future version of MATLAB

$\%$ handles empty - handles not created until after all CreateFcns called

$\%$ Hint: edit controls usually have a white background on Windows.

$\% \quad$ See ISPC and COMPUTER.

if ispc \&\& isequal(get(hObject,'BackgroundColor'), get(0,'defaultUicontrolBackgroundColor')) set(hObject,'BackgroundColor','white');

end

end

$\%$--- Executes on button press in Ipf_pushbutton.

function Ipf_pushbutton_Callback(hObject, , handles)

$\%$ hObject handle to Ipf_pushbutton (see GCBO)

$\%$ reserved - to be defined in a future version of MATLAB

$\%$ handles structure with handles and user data (see GUIDATA)

$\%$ samplesize = str2num(get(handles.num_sample_text,'String'));

$\%$ samplesize = get(handles.num_sample_text,'String');

$\%$ if strcmp(samplesize,") || num2str(samplesize) $<1$

$\%$

sample = get(handles.num_sample_text,'String');

samplesize $=$ str2num(sample);

if strcmp(sample,") == 1 || samplesize $<0$

errordlg('Please enter number of samples','Sample Number','modal');

return;

end

col = get(handles.lpf_pushbutton,'backg');

set(handles.Ipf_pushbutton,'str','RUNNING...','backg',[.2 .6 .6]);

pause(eps);

num1 = str2num(get(handles.lpf_text1,'String'));

num2 = str2num(get(handles.lpf_text2,'String'));

contents = get(handles.lpf_popup_dist,'String');

popupmenuvalue $=$ contents $\{$ get(handles.lpf_popup_dist,'Value')\};

cla(handles.axes1,'reset');

switch popupmenuvalue

case 'Normal'

pd = makedist('Normal','mu',num1,'sigma',num2);

$\mathrm{t}=$ truncate $(\mathrm{pd}, 0,1)$;

$\mathrm{n}=$ random(t,samplesize, 1$)$;

axes(handles.axes1)

nbins $=\max (\min ($ length $(n) \cdot / 10,100), 50)$;

$x i=$ linspace $(\min (n), \max (n), n b i n s)$;

$\mathrm{dx}=\operatorname{mean}(\operatorname{diff}(\mathrm{xi}))$;

$f i=\operatorname{histc}(n, x i-d x)$; 


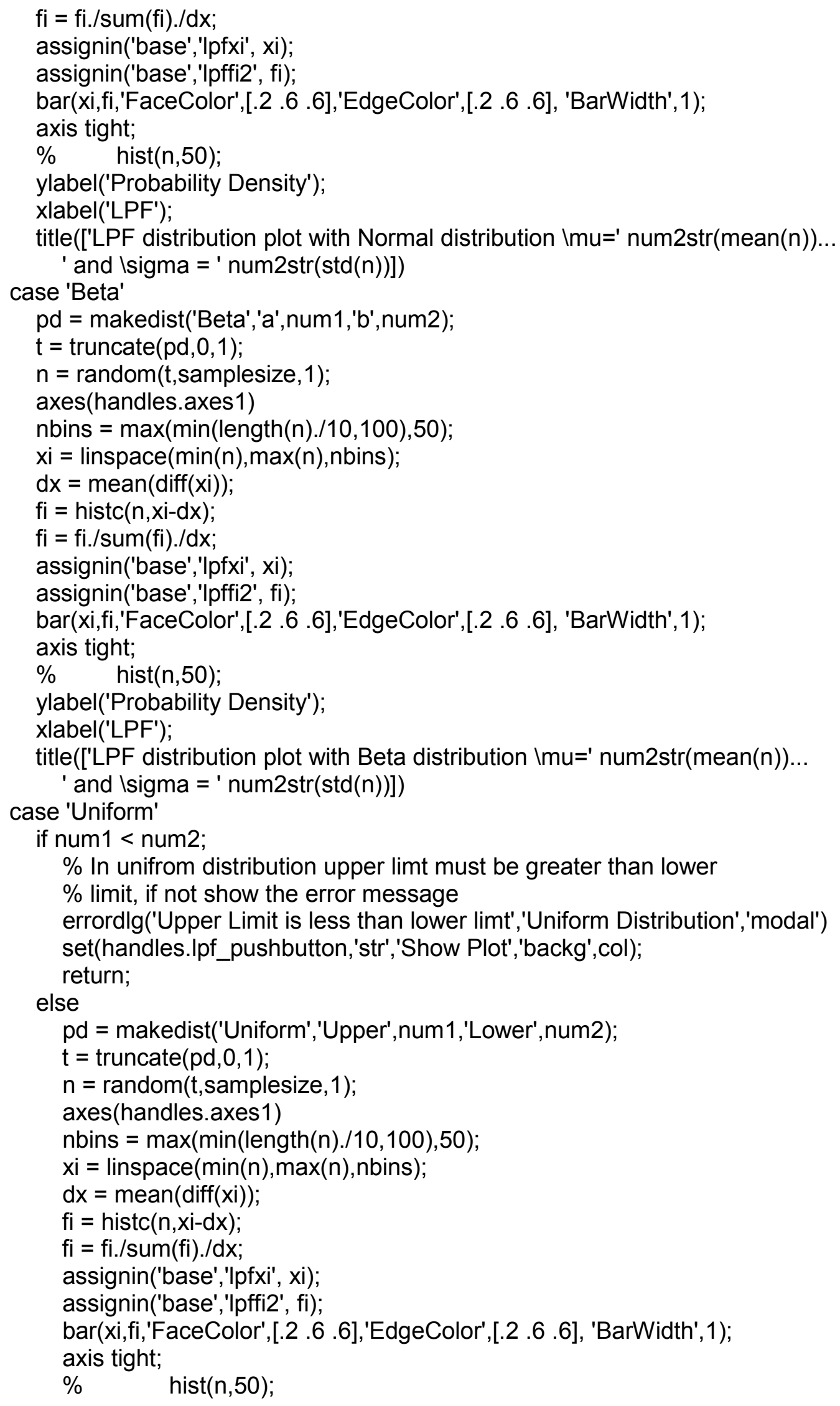




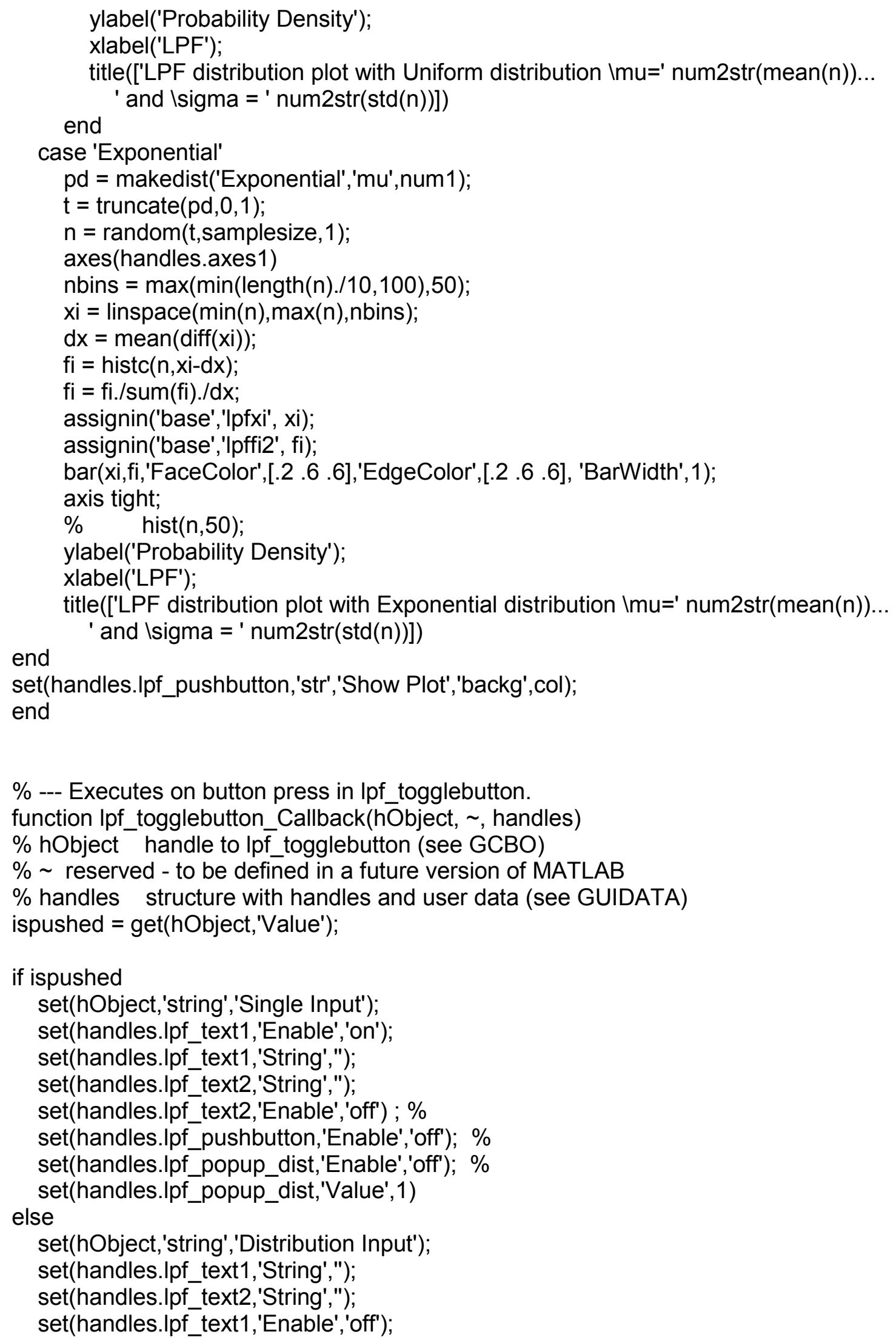


set(handles.Ipf_text2,'Enable','off'); \%

$\%$ set(handles.dr_pushbutton,'Enable','on'); \%

set(handles.Ipf_popup_dist,'Enable','on'); \%

end

end

\% Hint: get(hObject,'Value') returns toggle state of Ipf_togglebutton

$\%$--- Executes on button press in arf_pushbutton.

function arf_pushbutton_Callback(hObject, , handles)

$\%$ hObject handle to arf_pushbutton (see GCBO)

$\%$ reserved - to be defined in a future version of MATLAB

$\%$ handles structure with handles and user data (see GUIDATA)

sample = get(handles.num_sample_text,'String');

samplesize $=$ str2num(sample);

if strcmp(sample,") == 1 || samplesize $<0$

errordlg('Please enter number of samples','Sample Number','modal');

return;

end

col = get(handles.arf_pushbutton,'backg');

set(handles.arf_pushbutton,'str','RUNNING...','backg',[.2 .6 .6]);

pause(eps);

num1 = str2num(get(handles.arf_text1,'String'));

num2 = str2num(get(handles.arf_text2,'String'));

contents = get(handles.arf_popup_dist,'String');

popupmenuvalue = contents $\{$ get(handles.arf_popup_dist,'Value')\};

cla(handles.axes1,'reset');

switch popupmenuvalue

case 'Normal'

pd = makedist('Normal','mu',num1,'sigma',num2);

$\mathrm{t}=$ truncate $(\mathrm{pd}, 0$, inf $)$;

$\mathrm{n}=$ random $(\mathrm{t}$, samplesize, 1$)$;

axes(handles.axes1)

nbins $=\max (\min ($ length $(\mathrm{n}) \cdot / 10,100), 50)$;

$x i=$ linspace $(\min (n), \max (n)$, nbins $)$;

$\mathrm{dx}=\operatorname{mean}(\operatorname{diff}(\mathrm{xi}))$;

$\mathrm{fi}=\operatorname{histc}(\mathrm{n}, \mathrm{xi}-\mathrm{dx})$;

$\mathrm{fi}=\mathrm{fi} . / \mathrm{sum}(\mathrm{fi}) . / \mathrm{dx}$;

assignin('base','arfxi', xi);

assignin('base','arffi2', fi);

bar(xi,fi,'FaceColor',[.2 .6 .6],'EdgeColor',[.2 .6 .6], 'BarWidth',1);

axis tight;

$\% \quad$ hist(n,50);

ylabel('Probability Density');

xlabel('ARF'); 


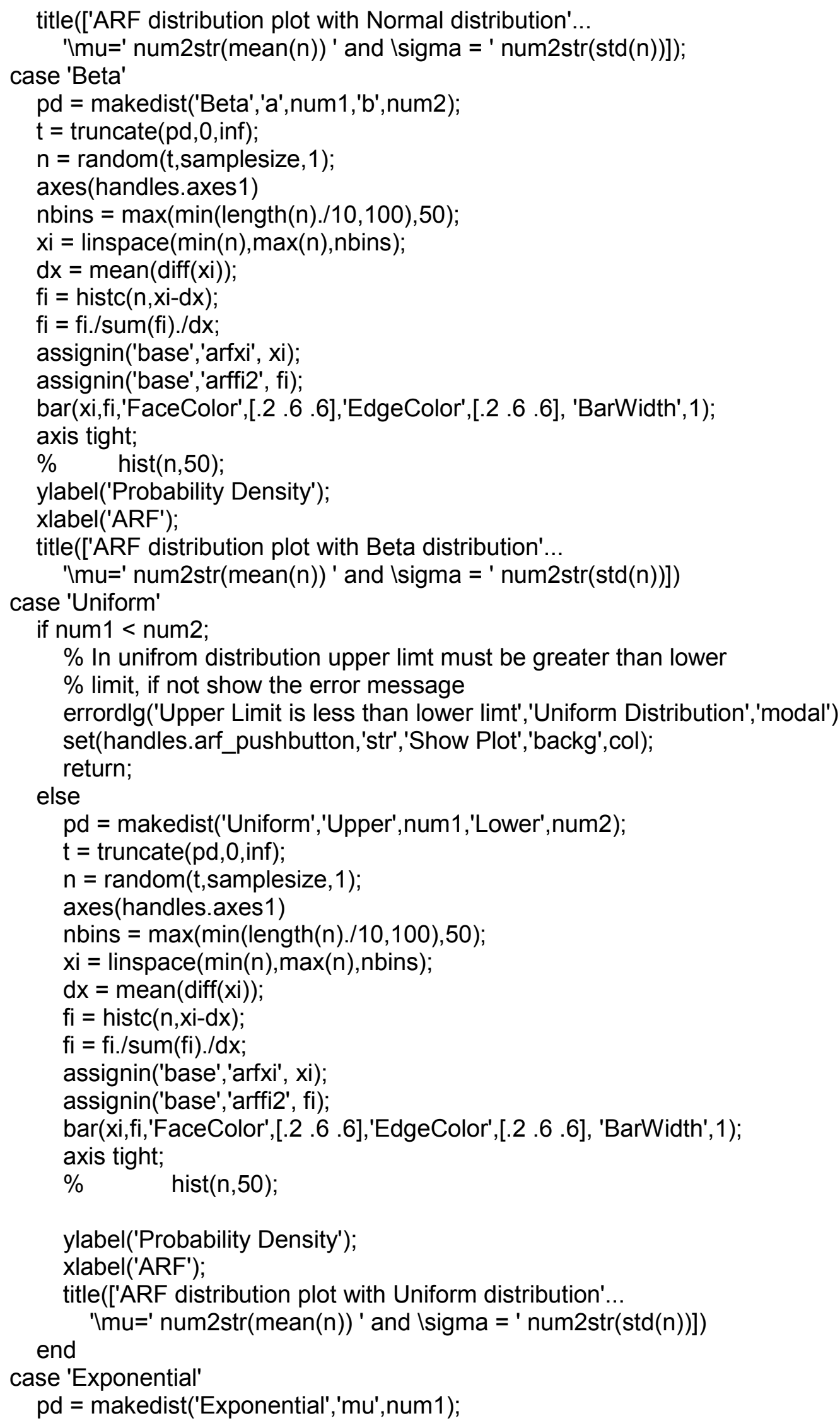


$\mathrm{t}=$ truncate $(\mathrm{pd}, 0, \mathrm{inf})$

$\mathrm{n}=$ random $(\mathrm{t}$, samplesize, 1$)$;

axes(handles.axes1)

$\mathrm{nbins}=\max (\min ($ length $(\mathrm{n}) \cdot / 10,100), 50)$;

$x i=$ linspace $(\min (n), \max (n), n b i n s)$;

$\mathrm{dx}=\operatorname{mean}(\operatorname{diff}(\mathrm{xi}))$;

$\mathrm{fi}=\operatorname{histc}(\mathrm{n}, \mathrm{xi}-\mathrm{dx})$;

$\mathrm{fi}=\mathrm{fi} . / \mathrm{sum}(\mathrm{fi}) . / \mathrm{dx}$;

assignin('base','arfxi', xi);

assignin('base','arffi2', fi);

bar(xi,fi,'FaceColor',[.2 .6 .6],'EdgeColor',[.2 .6 .6], 'BarWidth',1);

axis tight;

$\% \quad$ hist(n,50);

ylabel('Probability Density');

xlabel('ARF');

title(['ARF distribution plot with Exponential distribution'...

end

'Imu=' num2str(mean(n)) ' and Isigma = ' num2str(std(n))]);

set(handles.arf_pushbutton,'str','Show Plot','backg',col);

end

function arf_text2_Callback(hObject, , handles)

$\%$ hObject handle to arf text2 (see GCBO)

$\% \sim$ reserved - to be defined in a future version of MATLAB

$\%$ handles structure with handles and user data (see GUIDATA)

end

$\%$ Hints: get(hObject,'String') returns contents of arf_text2 as text

$\% \quad$ str2double(get(hObject,'String')) returns contents of arf_text2 as a double

$\%$--- Executes during object creation, after setting all properties.

function arf_text2_CreateFcn(hObject, , handles)

$\%$ hObject handle to arf_text2 (see GCBO)

$\% \sim$ reserved - to be defined in a future version of MATLAB

$\%$ handles empty - handles not created until after all CreateFcns called

$\%$ Hint: edit controls usually have a white background on Windows.

$\% \quad$ See ISPC and COMPUTER.

if ispc \&\& isequal(get(hObject,'BackgroundColor'), get(0,'defaultUicontrolBackgroundColor')) set(hObject,'BackgroundColor','white');

end

end

function arf_text1_Callback(hObject, , handles)

$\%$ hObject handle to arf_text1 (see GCBO)

$\%$ reserved - to be defined in a future version of MATLAB

$\%$ handles structure with handles and user data (see GUIDATA)

end 
$\%$ Hints: get(hObject,'String') returns contents of arf_text1 as text

$\% \quad$ str2double(get(hObject,'String')) returns contents of arf_text1 as a double

$\%$--- Executes during object creation, after setting all properties.

function arf_text1_CreateFcn(hObject, , handles)

$\%$ hObject handle to arf_text1 (see GCBO)

$\% \sim$ reserved - to be defined in a future version of MATLAB

$\%$ handles empty - handles not created until after all CreateFcns called

$\%$ Hint: edit controls usually have a white background on Windows.

$\% \quad$ See ISPC and COMPUTER.

if ispc \&\& isequal(get(hObject,'BackgroundColor'), get(0,'defaultUicontrolBackgroundColor')) set(hObject,'BackgroundColor','white');

end

end

$\%$--- Executes on selection change in arf_popup_dist.

function arf_popup_dist_Callback(hObject, , handles)

$\%$ hObject handle to arf_popup_dist (see GCBO)

$\%$ reserved - to be defined in a future version of MATLAB

$\%$ handles structure with handles and user data (see GUIDATA)

contents $=$ cellstr(get(hObject,'String'));

arfpopchoice $=$ contents $\{$ get $($ hObject, 'Value' $)\}$;

switch arfpopchoice

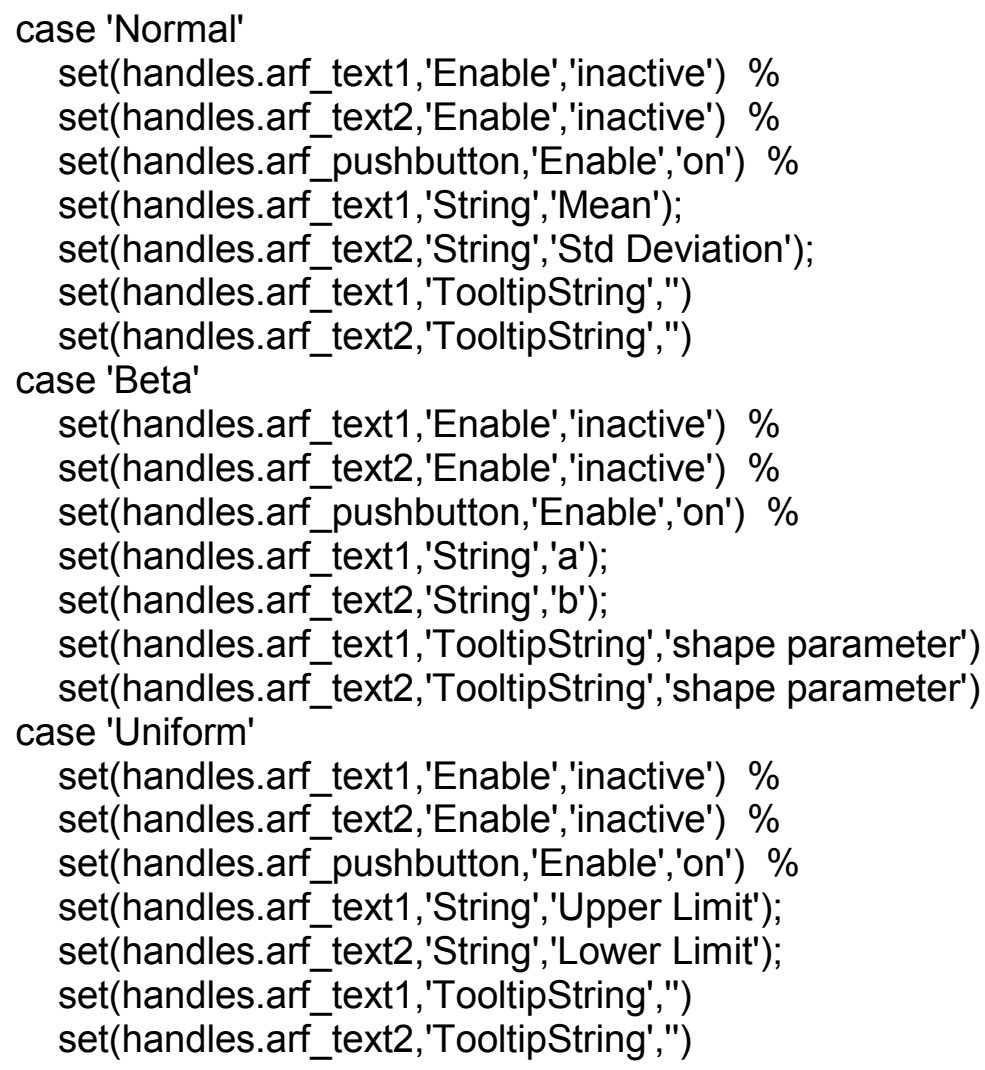




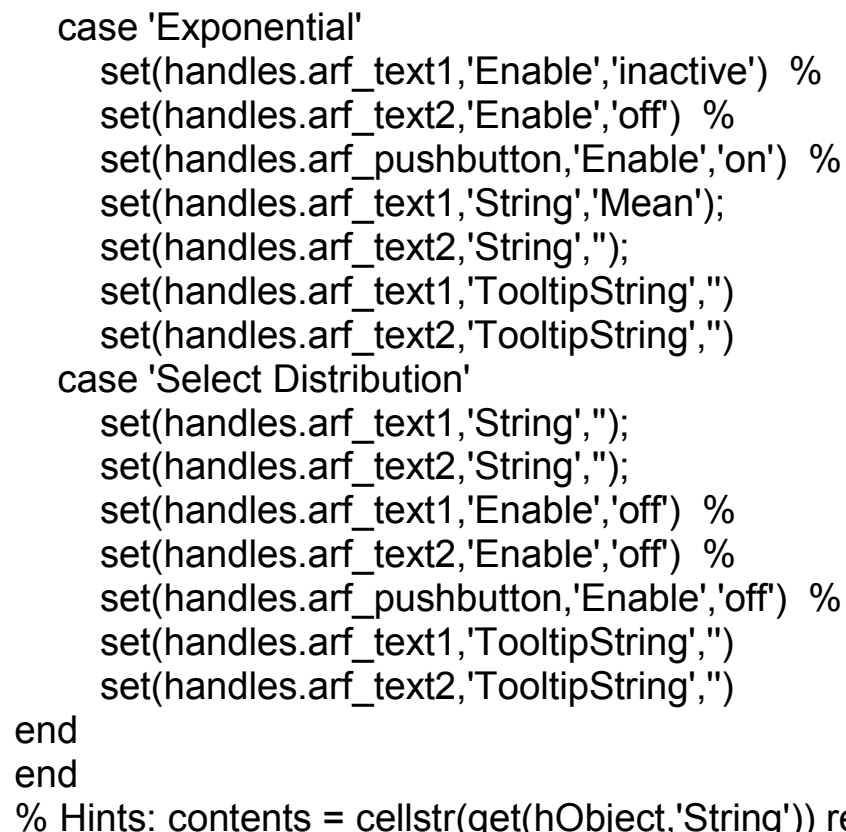

$\%$ Hints: contents = cellstr(get(hObject,'String')) returns arf_popup_dist contents as cell array

$\% \quad$ contents $\{$ get(hObject,'Value')\} returns selected item from arf_popup_dist

$\%$--- Executes during object creation, after setting all properties.

function arf_popup_dist_CreateFcn(hObject, , handles)

$\%$ hObject handle to arf popup_dist (see GCBO)

$\% \sim$ reserved - to be defined in a future version of MATLAB

$\%$ handles empty - handles not created until after all CreateFcns called

$\%$ Hint: popupmenu controls usually have a white background on Windows.

$\% \quad$ See ISPC and COMPUTER.

if ispc \&\& isequal(get(hObject,'BackgroundColor'), get(0,'defaultUicontrolBackgroundColor')) set(hObject,'BackgroundColor','white');

end

end

$\%$--- Executes on button press in arf_togglebutton.

function arf_togglebutton_Callback(hŌbject, , handles)

$\%$ hObject handle to arf_togglebutton (see GCBO)

$\% \sim$ reserved - to be defined in a future version of MATLAB

$\%$ handles structure with handles and user data (see GUIDATA)

ispushed = get(hObject,'Value');

if ispushed

set(hObject,'string','Single Input');

set(handles.arf_text1,'Enable','on');

set(handles.arf_text1,'String',");

set(handles.arf_text2,'String',");

set(handles.arf_text2,'Enable','off') ; \%

set(handles.arf_pushbutton,'Enable','off'); \%

set(handles.arf_popup_dist,'Enable','off'); \%

set(handles.arf_popup_dist,'Value',1) 


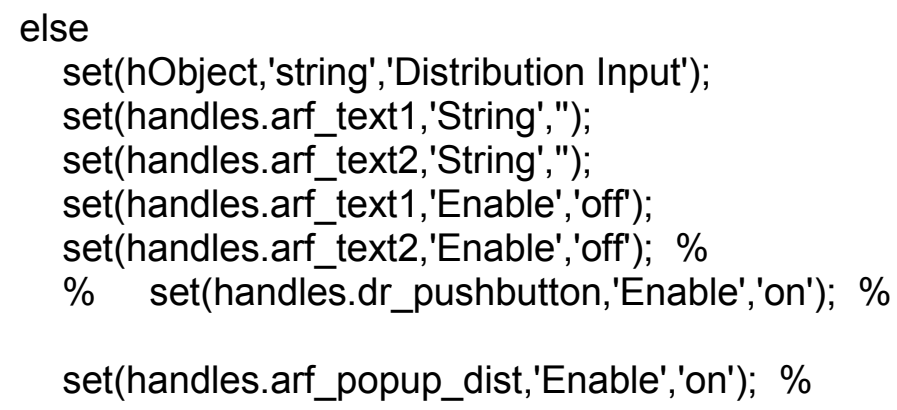




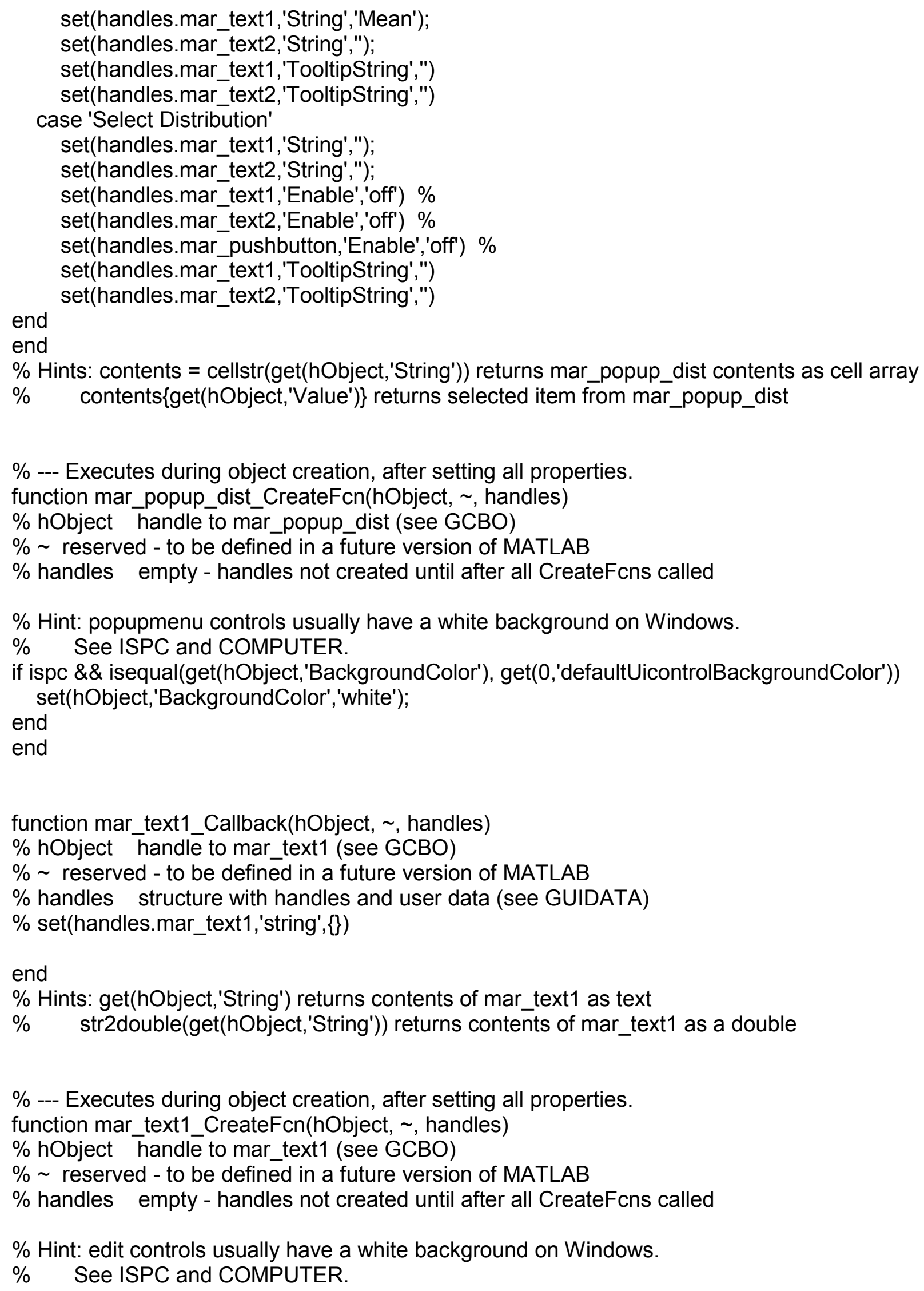


if ispc \&\& isequal(get(hObject,'BackgroundColor'), get(0,'defaultUicontrolBackgroundColor')) set(hObject,'BackgroundColor','white');

end

end

function mar_text2_Callback(hObject, , handles)

$\%$ hObject handle to mar_text2 (see GCBO)

$\% \sim$ reserved - to be defined in a future version of MATLAB

$\%$ handles structure with handles and user data (see GUIDATA)

$\%$ Hints: get(hObject,'String') returns contents of mar_text2 as text

$\% \quad$ str2double(get(hObject,'String')) returns contents of mar_text2 as a double

end

$\%$--- Executes during object creation, after setting all properties.

function mar_text2_CreateFcn(hObject, , handles)

$\%$ hObject handle to mar_text2 (see GCBO)

$\% \sim$ reserved - to be defined in a future version of MATLAB

$\%$ handles empty - handles not created until after all CreateFcns called

$\%$ Hint: edit controls usually have a white background on Windows.

$\% \quad$ See ISPC and COMPUTER.

if ispc \&\& isequal(get(hObject,'BackgroundColor'), get( 0 ,'defaultUicontrolBackgroundColor')) set(hObject,'BackgroundColor','white');

end

end

$\%$--- Executes on button press in mar_pushbutton.

function mar_pushbutton_Callback(hÖbject, $\sim$, handles)

$\%$ hObject handle to mar_pushbutton (see GCBO)

$\% \sim$ reserved - to be defined in a future version of MATLAB

$\%$ handles structure with handles and user data (see GUIDATA)

sample = get(handles.num_sample_text,'String');

samplesize $=$ str2num(sample);

if strcmp(sample,") == 1 || samplesize $<0$

errordlg('Please enter number of samples','Sample Number','modal'); return;

end

col = get(handles.mar_pushbutton,'backg');

set(handles.mar_pushbutton,'str','RUNNING...','backg',[.2 .6 .6]);

pause(eps);

num1 = str2num(get(handles.mar_text1,'String'));

num2 = str2num(get(handles.mar_text2,'String'));

contents = get(handles.mar_popup_dist,'String');

popupmenuvalue $=$ contents $\{$ get(handles.mar_popup_dist,'Value')\};

cla(handles.axes1,'reset');

switch popupmenuvalue 


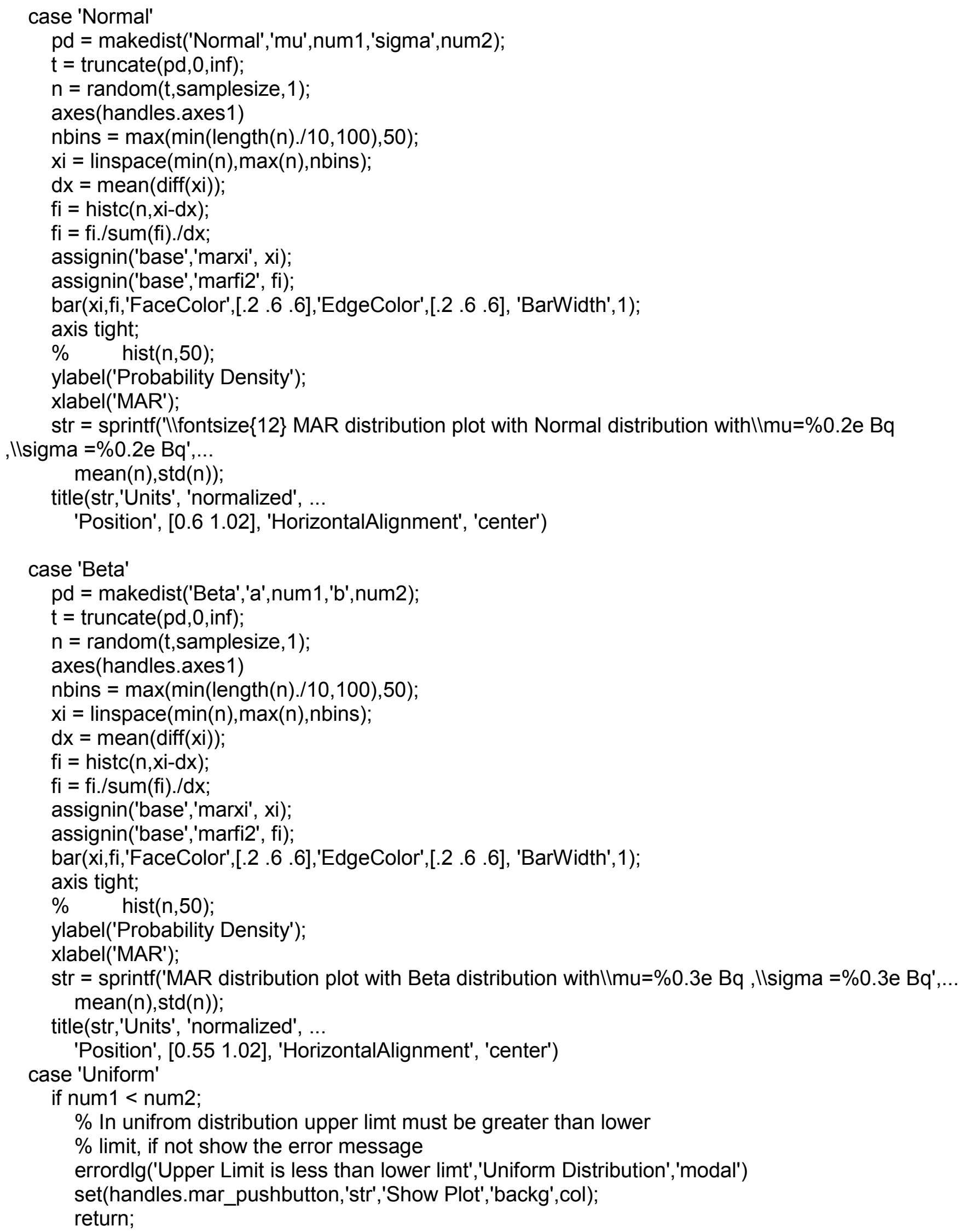




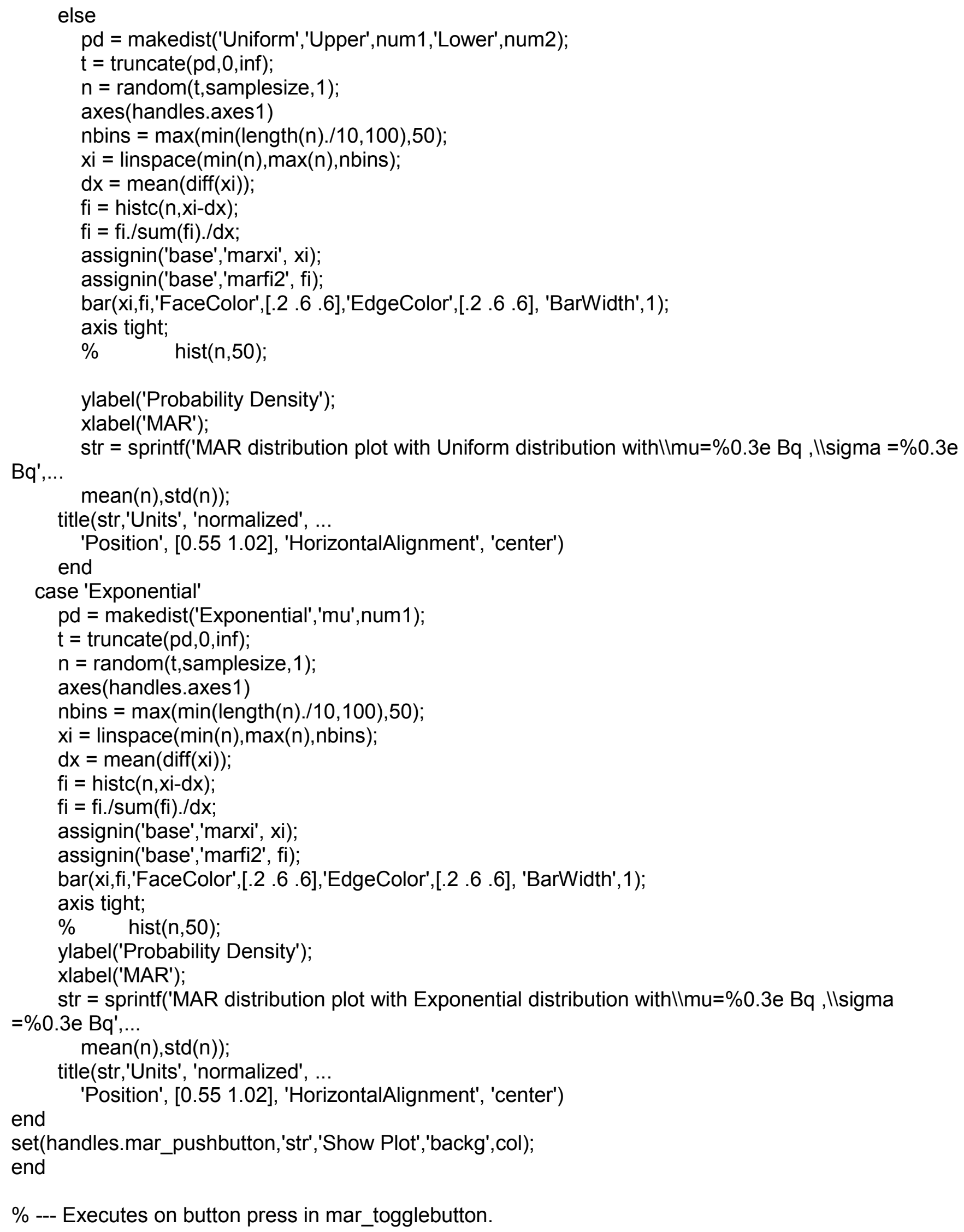




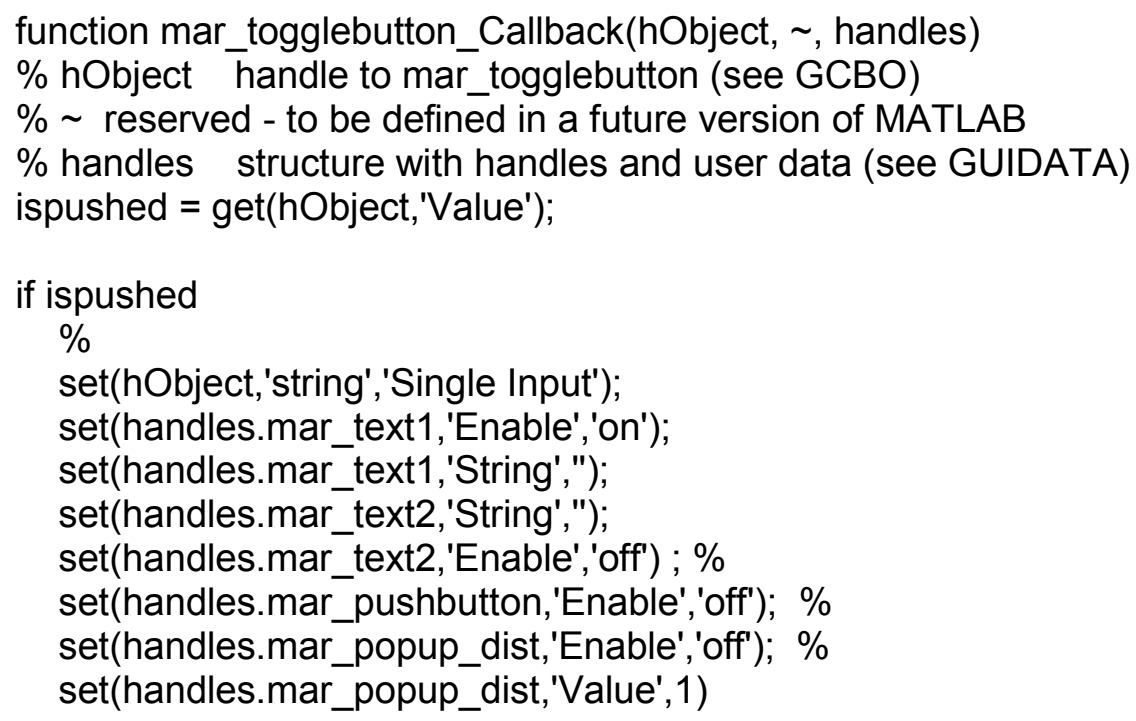

else

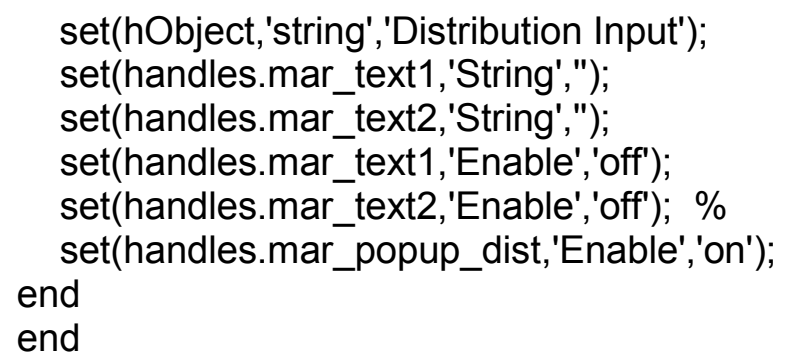

\% Hint: get(hObject,'Value') returns toggle state of mar_togglebutton

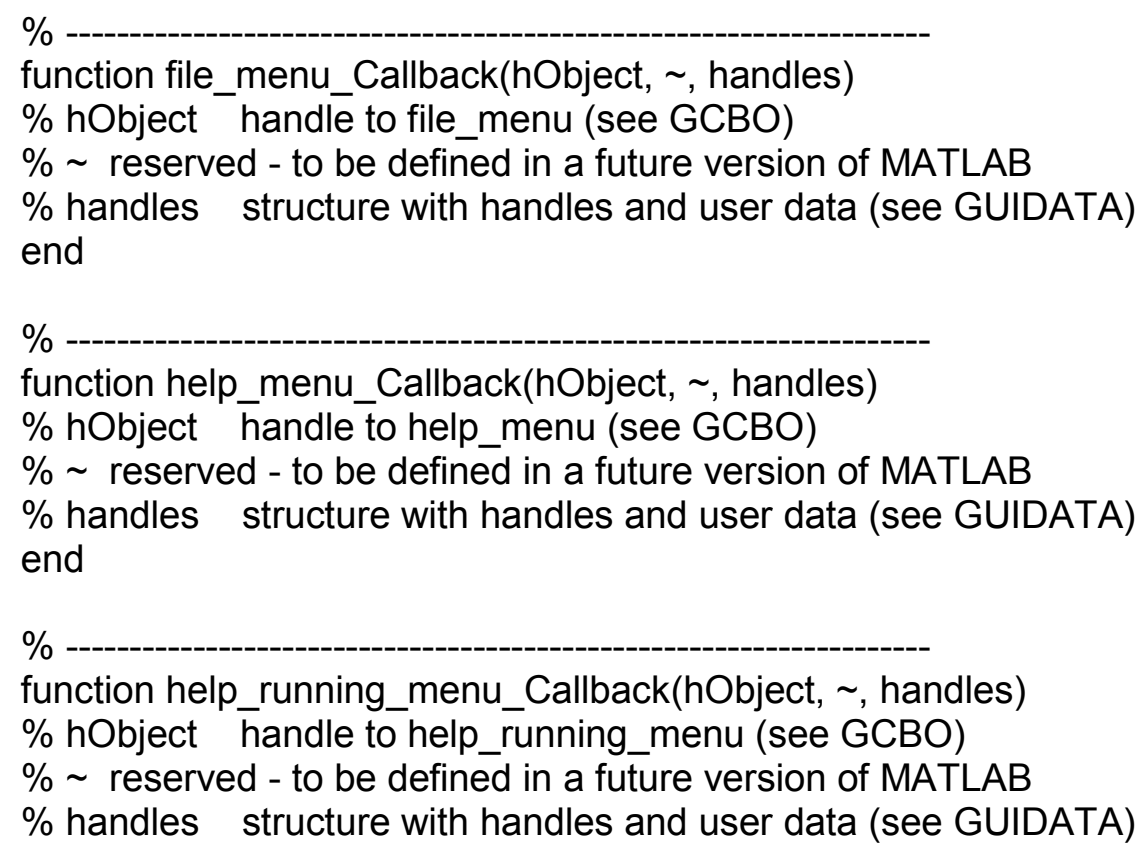


\%for windows system

$\%$ filename = 'C:IProgram Files ISUISODAlapplicationlhelp.pdf';

$\%$ winopen('C:IProgram FilesIISUISODAlapplicationlhelp.pdf')

$\%$

$\%$ mac system

system('open /Applications/ISU/SODA/application/help.pdf')

$\%$ system('open help.pdf' );

end

$\%$

function about_menu_Callback(hObject, $\sim$, handles)

$\%$ hObject handle to about_menu (see GCBO)

$\% \sim$ reserved - to be defined in a future version of MATLAB

$\%$ handles structure with handles and user data (see GUIDATA)

About;

\% msgbox(\{'Dr. Chad Pope, Idaho State University' ' ' 'Jason Andrus, Idaho National Lab'...

$\%$ ' ' 'Graduate Student' ' ' 'Kushal Bhattarai, Idaho State University',...

$\% \quad$ ' ' 'Undergraduate Students' ' ' 'Abdullah Alomani' ' ' 'Abraham Chupp'...

$\% \quad$ ' ' 'Mason Jaussi'\},'About');

end

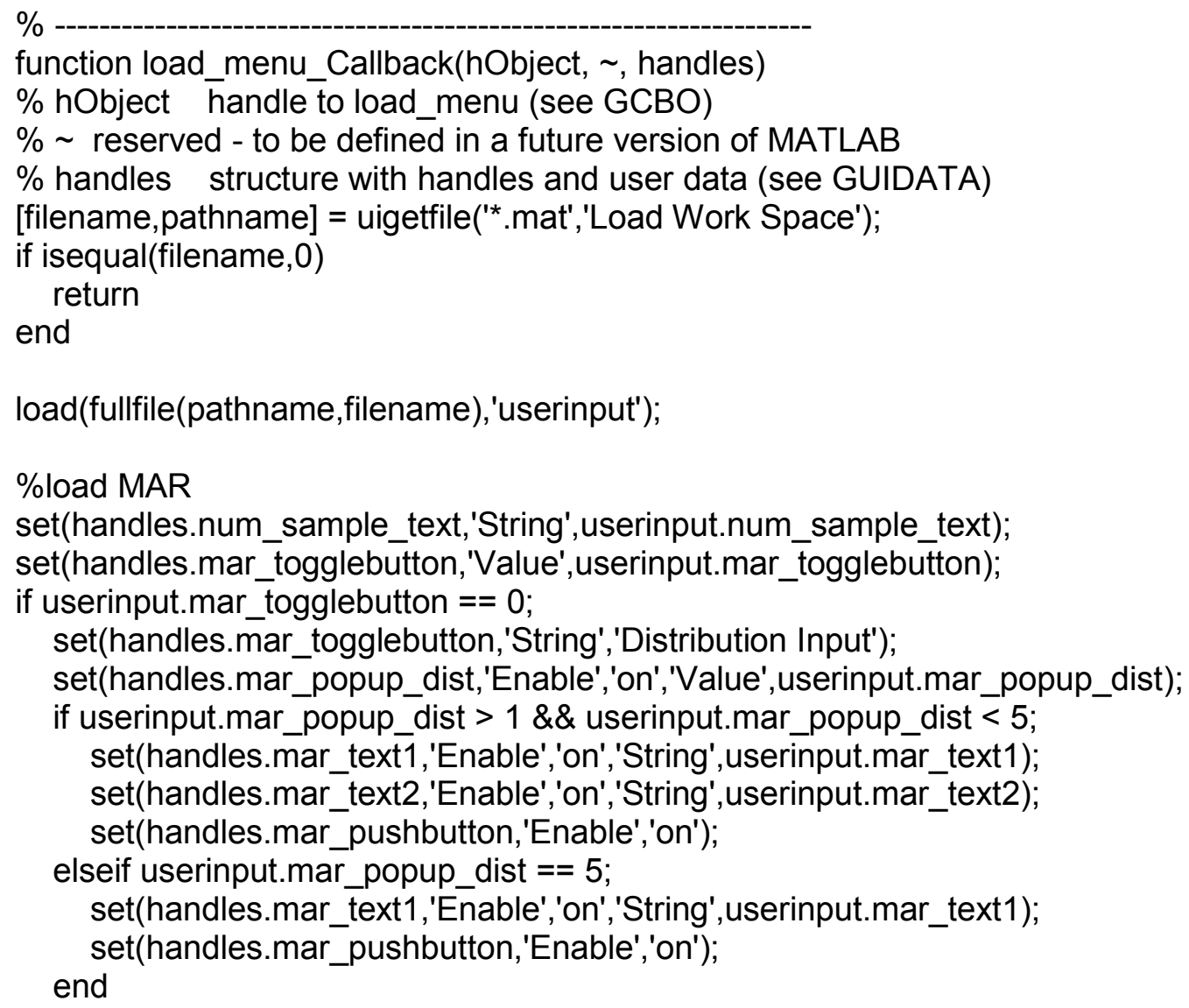




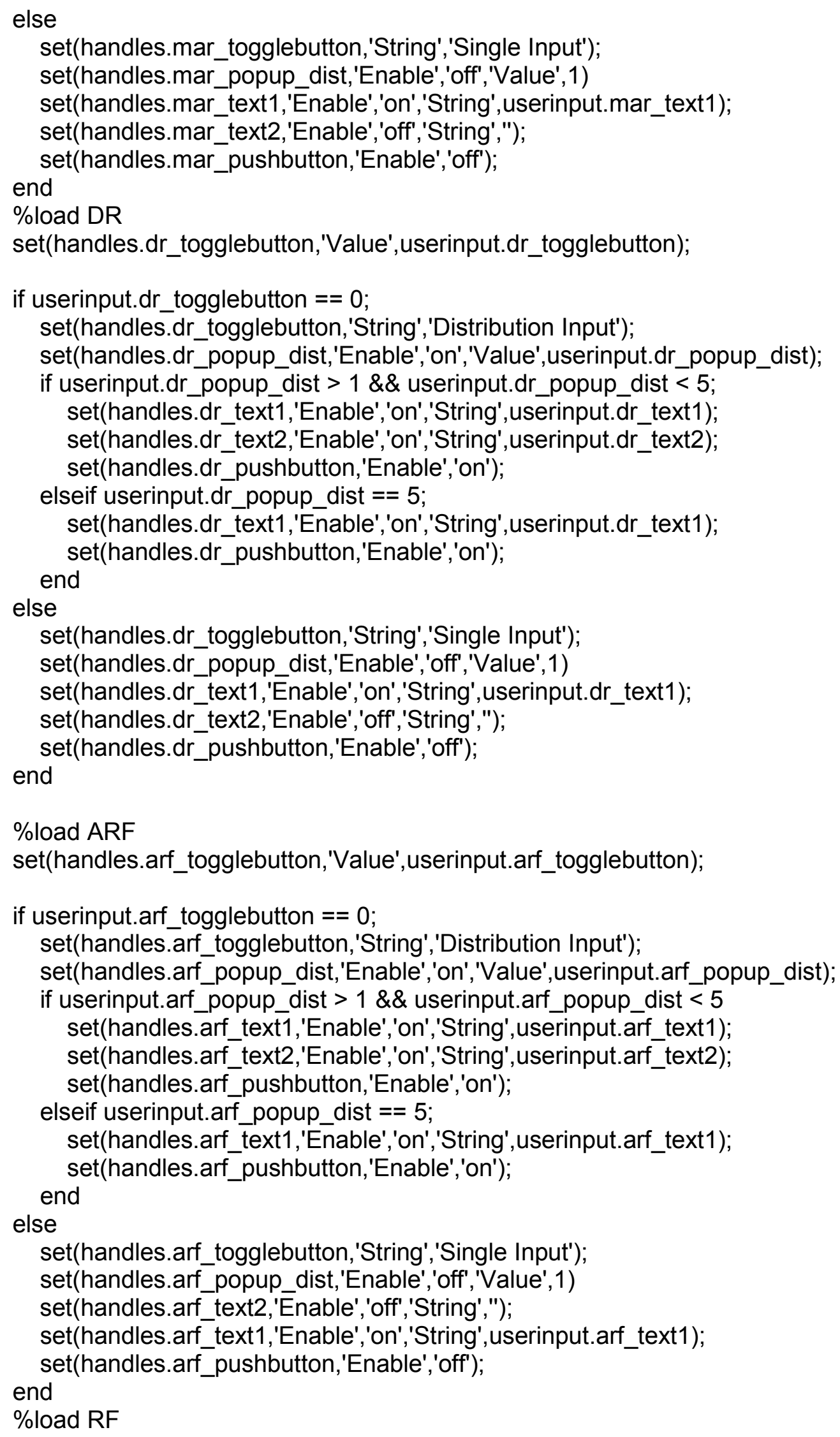




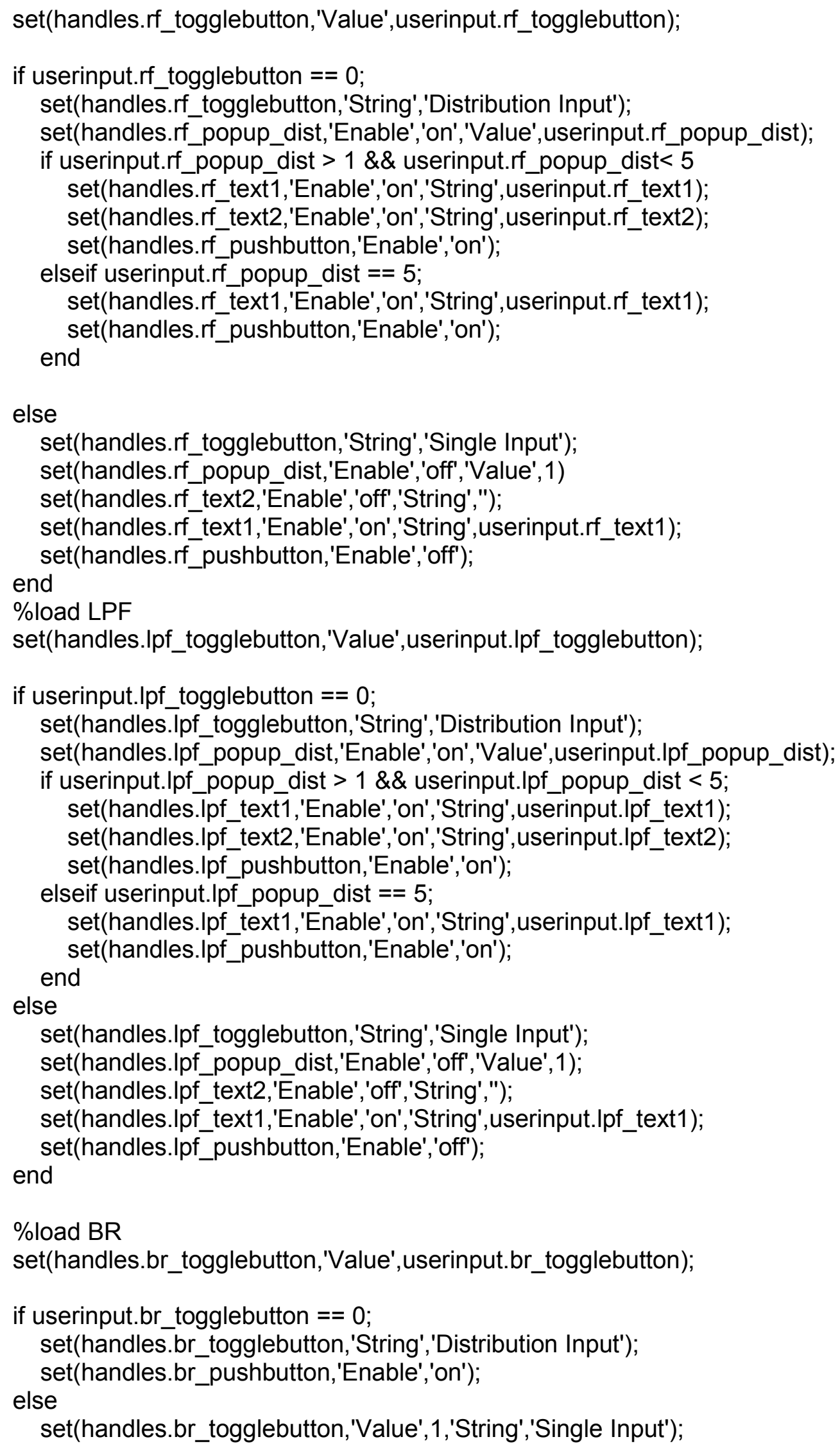


set(handles.br_text1,'Enable','on','String',userinput.br_text1);

set(handles.br_pushbutton,'Enable','off');

end

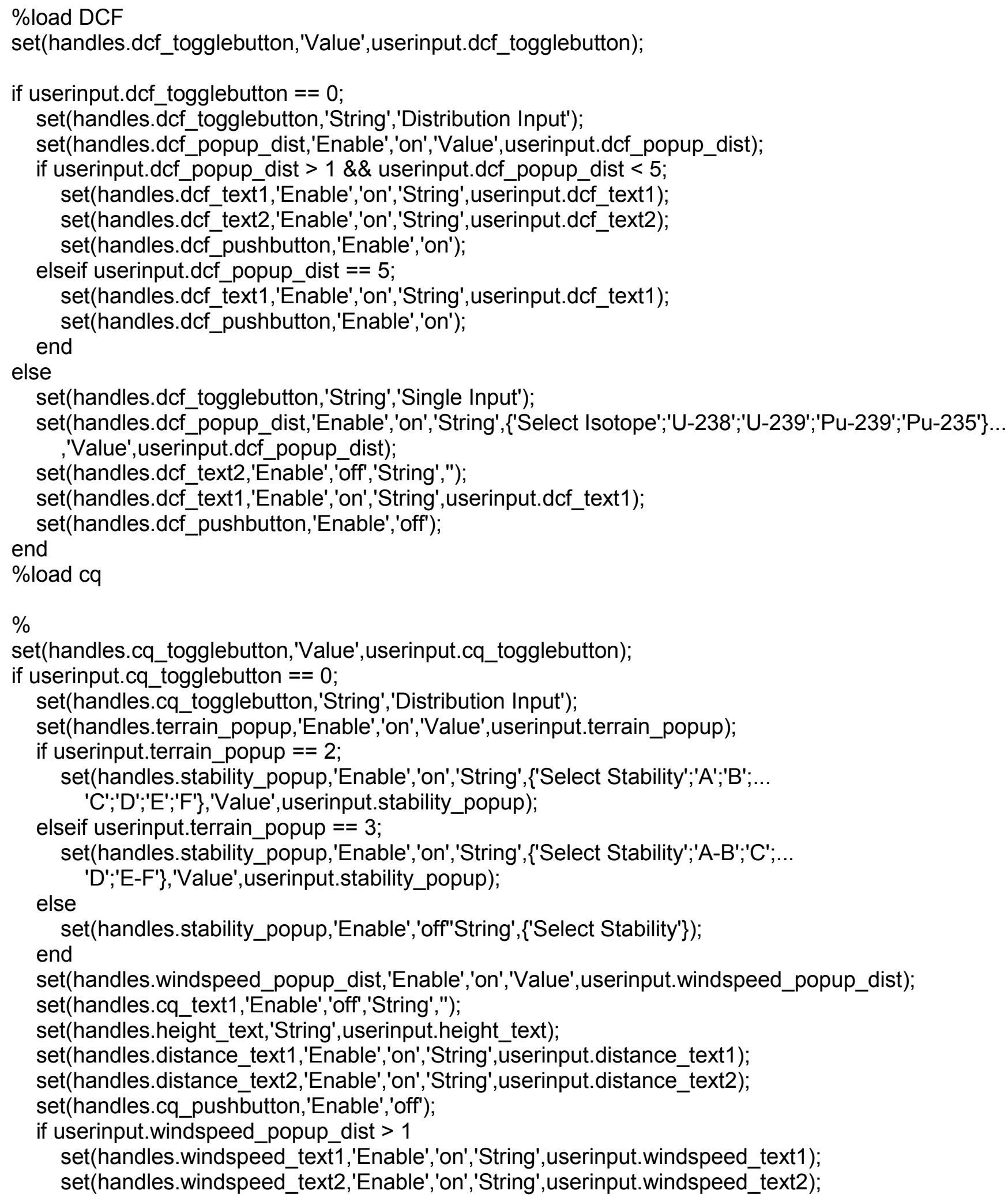




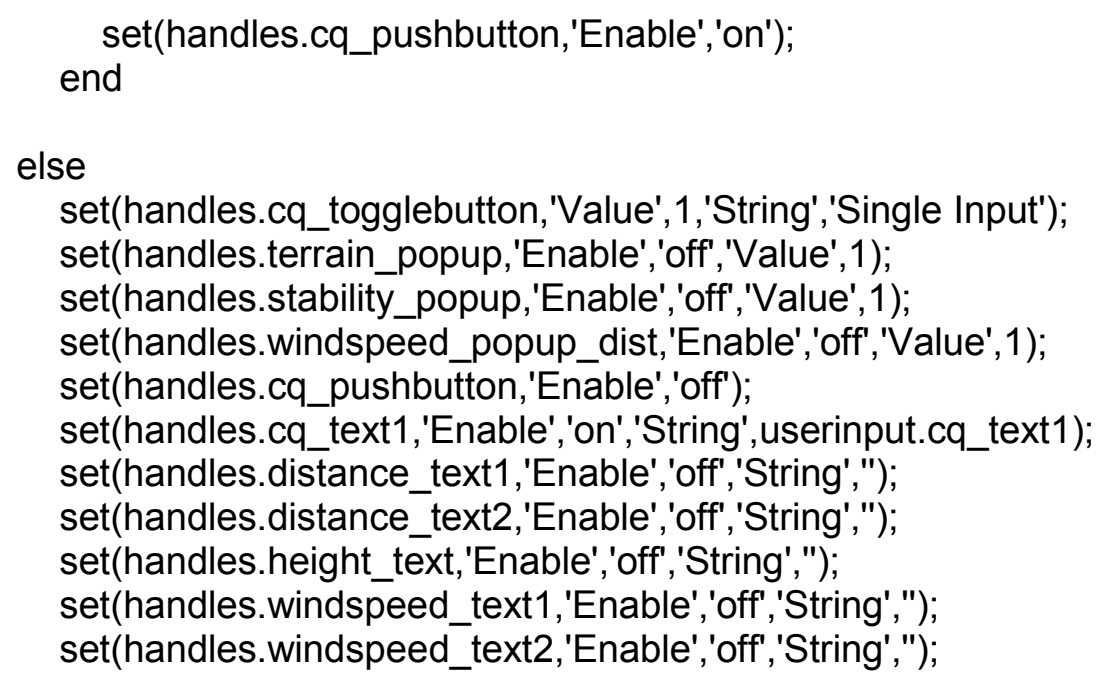

end

end

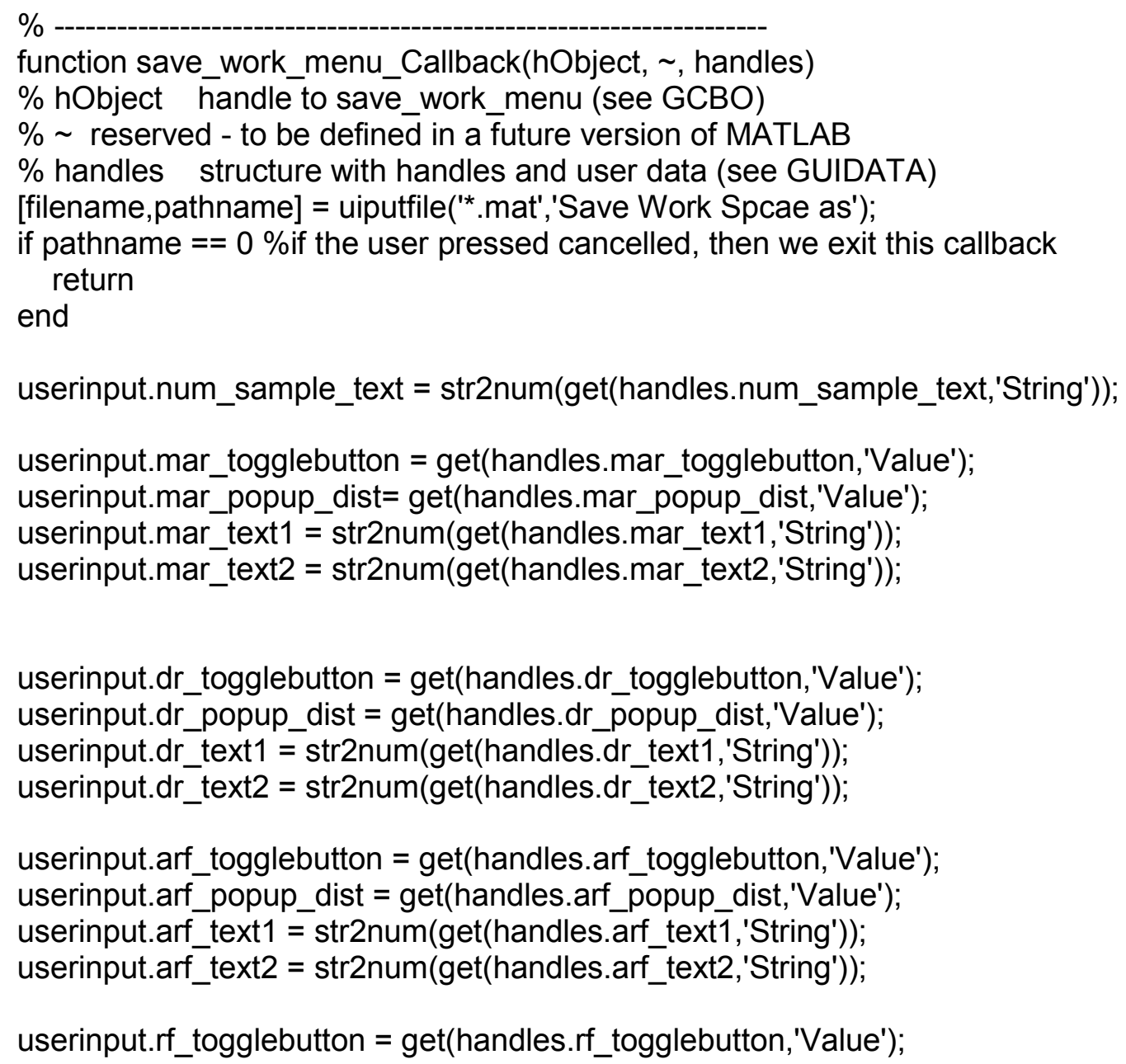


userinput.rf_popup_dist = get(handles.rf_popup_dist,'Value'); userinput.rf_text1 = str2num(get(handles.rf_text1,'String')); userinput.rf_text2 = str2num(get(handles.rf_text2,'String'));

userinput.Ipf_togglebutton = get(handles.lpf_togglebutton, 'Value'); userinput.Ipf_popup_dist = get(handles.Ipf_popup_dist,'Value'); userinput.Ipf_text1 = str2num(get(handles.Ipf_text1,'String')); userinput.lpf_text2 = str2num(get(handles.lpf_text2,'String'));

userinput.br_togglebutton = get(handles.br_togglebutton,'Value'); userinput.br_text1 = str2num(get(handles.br_text1,'String'));

userinput.dcf_togglebutton = get(handles.dcf_togglebutton,'Value'); userinput.dcf_popup_dist =get(handles.dcf_popup_dist,'Value'); userinput.dcf_text1 = str2num(get(handles.dcf_text1,'String')); userinput.dcf_text2 = str2num(get(handles.dcf_text2,'String'));

userinput.cq_togglebutton = get(handles.cq_togglebutton,'Value'); userinput.distance_text1 = str2num(get(handles.distance_text1,'String')); userinput.distance_text2 = str2num(get(handles.distance_text2,'String')); userinput.terrain_popup = get(handles.terrain_popup,'Value'); userinput.stability_popup = get(handles.stability_popup,'Value'); userinput.windspeed_popup_dist = get(handles.windspeed_popup_dist,'Value'); userinput.cq_text $1=\mathbf{s t r} 2 \mathrm{num}(\mathrm{get}($ handles.cq_text1,'String'));

userinput.windspeed_text1= str2num(get(handles.windspeed_text1,'String')); userinput.windspeed_text2=str2num(get(handles.windspeed_text2,'String')); userinput.height_text = str2num(get(handles.height_text,'String'));

save(fullfile(pathname, filename),'userinput')

end

\section{$\%$}

function save_image_menu_Callback(hObject, , handles)

$\%$ hObject handle to save_image_menu (see GCBO)

$\% \sim$ reserved - to be defined in a future version of MATLAB

$\%$ handles structure with handles and user data (see GUIDATA)

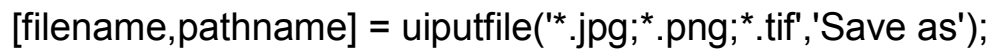

if pathname $==0 \%$ if the user pressed cancelled, then we exit this callback return

end

haxes=handles.axes 1 ;

ftmp = figure('visible','off');

new_axes $=$ copyobj(haxes, ftmp);

set(new_axes,'Units','normalized','Position',[0.1 0.10 .8 0.8]); 
saveas(ftmp, fullfile(pathname,filename)); delete(ftmp);

end

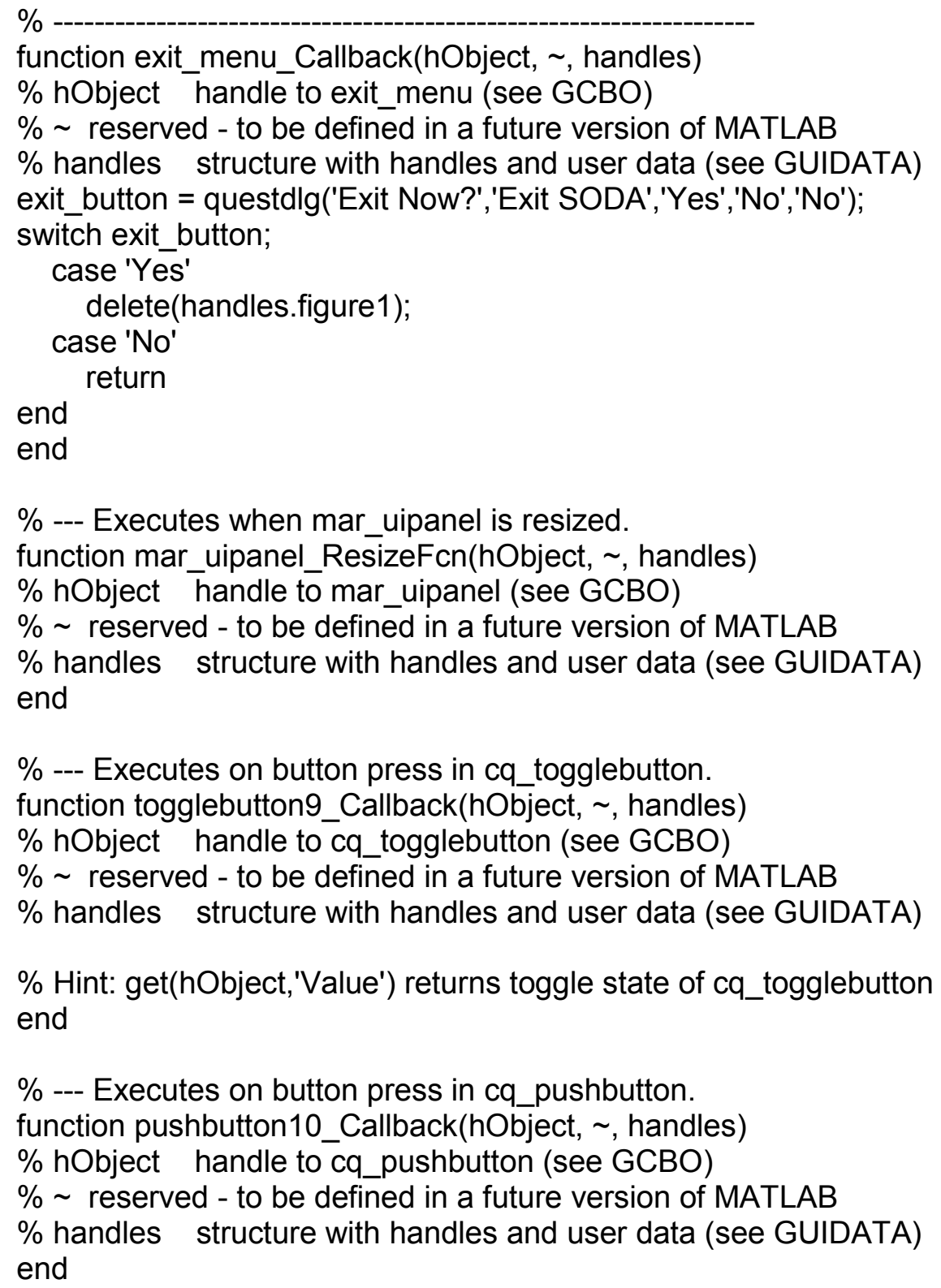


end

$\%$--- Executes during object creation, after setting all properties.

function cq_text_CreateFcn(hObject, , handles)

$\%$ hObject handle to cq_text1 (see GCBO)

$\%$ reserved - to be defined in a future version of MATLAB

$\%$ handles empty - handles not created until after all CreateFcns called

$\%$ Hint: edit controls usually have a white background on Windows.

$\% \quad$ See ISPC and COMPUTER.

if ispc \&\& isequal(get(hObject,'BackgroundColor'), get(0,'defaultUicontrolBackgroundColor')) set(hObject,'BackgroundColor','white');

end

end

function distance_text2_Callback(hObject, , handles)

$\%$ hObject handle to distance_text2 (see GCBO)

$\% \sim$ reserved - to be defined in a future version of MATLAB

$\%$ handles structure with handles and user data (see GUIDATA)

$\%$ Hints: get(hObject,'String') returns contents of distance_text2 as text

$\% \quad$ str2double(get(hObject,'String')) returns contents of distance_text2 as a double

end

$\%$--- Executes during object creation, after setting all properties.

function distance_text2_CreateFcn(hObject, , handles)

$\%$ hObject handle to distance_text2 (see GCBO)

$\% \sim$ reserved - to be defined in a future version of MATLAB

$\%$ handles empty - handles not created until after all CreateFcns called

$\%$ Hint: edit controls usually have a white background on Windows.

$\% \quad$ See ISPC and COMPUTER.

if ispc \&\& isequal(get(hObject,'BackgroundColor'), get(0,'defaultUicontrolBackgroundColor')) set(hObject,'BackgroundColor','white');

end

end

function distance_text1_Callback(hObject, , handles)

$\%$ hObject handle to distance_text1 (see GCBO)

$\% \sim$ reserved - to be defined in a future version of MATLAB

$\%$ handles structure with handles and user data (see GUIDATA)

$\%$ Hints: get(hObject,'String') returns contents of distance_text1 as text

$\% \quad$ str2double(get(hObject,'String')) returns contents of distance_text1 as a double

end

$\%$--- Executes during object creation, after setting all properties.

function distance_text1_CreateFcn(hObject, , handles)

$\%$ hObject handle to distance_text1 (see GCBO) 
$\%$ reserved - to be defined in a future version of MATLAB

$\%$ handles empty - handles not created until after all CreateFcns called

$\%$ Hint: edit controls usually have a white background on Windows.

$\% \quad$ See ISPC and COMPUTER.

if ispc \&\& isequal(get(hObject,'BackgroundColor'), get(0,'defaultUicontrolBackgroundColor')) set(hObject,'BackgroundColor','white');

end

end

\% --- Executes on selection change in distance_popup_dist.

function distance_popup_dist_Callback(hObject, , handles)

$\%$ hObject handle to distance_popup_dist (see GCBO)

$\%$ reserved - to be defined in a future version of MATLAB

$\%$ handles structure with handles and user data (see GUIDATA)

contents = cellstr(get(hObject,'String'));

distance_popchoice $=$ contents $\{$ get $($ hObject, 'Value') $\}$;

switch distance_popchoice

case 'Normal'

set(handles.distance_text1,'Enable','inactive') \%

set(handles.distance_text2,'Enable','inactive') \%

set(handles.distance_text1,'String','Mean');

set(handles.distance_text2,'String','Std Deviation');

set(handles.cq_pushb̄utton,'Enable','off')

set(handles.distance_text1,'TooltipString',")

set(handles.distance_text2,'TooltipString',")

case 'Beta'

set(handles.distance_text1,'Enable','inactive') \%

set(handles.distance_text2,'Enable','inactive') \%

set(handles.distance_text1,'String','a');

set(handles.distance_text2,'String','b');

set(handles.distance_text1,'TooltipString','shape parameter')

set(handles.distance_text2,'TooltipString','shape parameter')

set(handles.cq_pushbutton,'Enable','off')

case 'Uniform'

set(handles.distance_text1,'Enable','inactive') \%

set(handles.distance_text2,'Enable','inactive') \%

set(handles.distance_text1,'String','Upper Limit');

set(handles.distance_text2,'String','Lower Limit');

set(handles.cq_pushbutton,'Enable','off')

set(handles.distance_text1,'TooltipString',")

set(handles.distance_text2,'TooltipString',")

case 'Exponential'

set(handles.distance_text1,'Enable','inactive') \%

set(handles.distance_text2,'Enable','off') \%

set(handles.distance_text1,'String','Mean');

set(handles.cq_pushbutton,'Enable','off')

set(handles.distance_text2,'String',");

set(handles.distance_text1,'TooltipString',")

set(handles.distance_text2,'TooltipString',")

case 'Select Distribution' 


$$
\begin{aligned}
& \text { set(handles.distance_text1,''String',"); } \\
& \text { set(handles.distance_text2,'String',"); } \\
& \text { set(handles.distance_text1,'Enable','off') \% } \\
& \text { set(handles.distance_text2,'Enable','off') \% } \\
& \text { set(handles.cq_pushbutton,'Enable','off') \% } \\
& \text { set(handles.distance_text1,'TooltipString',") } \\
& \text { set(handles.distance_text2,'TooltipString',") }
\end{aligned}
$$

end

end

$\%$ Hints: contents $=$ cellstr(get(hObject,'String')) returns distance_popup_dist contents as cell array

$\% \quad$ contents $\{$ get(hObject,'Value')\} returns selected item from distance_popup_dist

$\%$--- Executes during object creation, after setting all properties.

function distance_popup_dist_CreateFcn(hObject, , handles)

$\%$ hObject handle to distance_popup_dist (see GCBO)

$\% \sim$ reserved - to be defined in a future version of MATLAB

$\%$ handles empty - handles not created until after all CreateFcns called

\% Hint: popupmenu controls usually have a white background on Windows.

$\% \quad$ See ISPC and COMPUTER.

if ispc \&\& isequal(get(hObject,'BackgroundColor'), get( 0 ,'defaultUicontrolBackgroundColor')) set(hObject,'BackgroundColor','white');

end

end

function height_text_Callback(hObject, , handles)

$\%$ hObject handle to height_text (see GCBO)

$\% \sim$ reserved - to be defined in a future version of MATLAB

$\%$ handles structure with handles and user data (see GUIDATA)

$\%$ Hints: get(hObject,'String') returns contents of height_text as text

$\% \quad$ str2double(get(hObject,'String')) returns contents of height_text as a double

end

$\%$--- Executes during object creation, after setting all properties.

function height_text_CreateFcn(hObject, , handles)

$\%$ hObject handle to height_text (see GCBO)

$\% \sim$ reserved - to be defined in a future version of MATLAB

$\%$ handles empty - handles not created until after all CreateFcns called

$\%$ Hint: edit controls usually have a white background on Windows.

$\% \quad$ See ISPC and COMPUTER.

if ispc \&\& isequal(get(hObject,'BackgroundColor'), get(0,'defaultUicontrolBackgroundColor')) set(hObject,'BackgroundColor','white');

end

end 
$\%$--- Executes on selection change in stability_popup.

function stability_popup_Callback(hObject, $\sim$, handles)

$\%$ hObject handle to stability_popup (see GCBO)

$\% \sim$ reserved - to be defined in a future version of MATLAB

$\%$ handles structure with handles and user data (see GUIDATA)

$\%$ Hints: contents = cellstr(get(hObject,'String')) returns stability_popup contents as cell array

$\% \quad$ contents $\{$ get(hObject,'Value')\} returns selected item from stability_popup

end

$\%$--- Executes during object creation, after setting all properties.

function stability_popup_CreateFcn(hObject, , handles)

$\%$ hObject handle to stability_popup (see GCBO)

$\% \sim$ reserved - to be defined in a future version of MATLAB

$\%$ handles empty - handles not created until after all CreateFcns called

\% Hint: popupmenu controls usually have a white background on Windows.

$\% \quad$ See ISPC and COMPUTER.

if ispc \&\& isequal(get(hObject,'BackgroundColor'), get( 0 ,'defaultUicontrolBackgroundColor')) set(hObject,'BackgroundColor','white');

end

end

$\%$--- Executes on selection change in terrain_popup.

function terrain_popup_Callback(hObject, , handles)

$\%$ hObject handle to terrain_popup (see GCBO)

$\% \sim$ reserved - to be defined in a future version of MATLAB

$\%$ handles structure with handles and user data (see GUIDATA)

contents = cellstr(get(hObject,'String'));

terrain_popchoice = contents $\{$ get $($ hObject,'Value') $\}$;

switch terrain_popchoice

case 'Urban Area'

set(handles.stability_popup,'Enable','on')

set(handles.stability_popup,'String',\{'Select Stability';'A-B';'C';...

'D';'E-F'\},'Value', 1);

case 'Rural/Open Country'

set(handles.stability_popup,'Enable','on')

set(handles.stability_popup,'String',\{'Select Stability';'A';'B';,...

'C';'D';'E';'F'\},'Value', 1);

case 'Select Terrain'

end

set(handles.stability_popup,'Value', 1,'String','Select Stability','Enable','off');

end

$\%$ Hints: contents = cellstr(get(hObject,'String')) returns terrain_popup contents as cell array

$\% \quad$ contents $\{g e t($ hObject,'Value')\} returns selected item from terrain_popup

$\%$--- Executes during object creation, after setting all properties.

function terrain_popup_CreateFcn(hObject, , handles) 
$\%$ hObject handle to terrain_popup (see GCBO)

$\% \sim$ reserved - to be defined in a future version of MATLAB

$\%$ handles empty - handles not created until after all CreateFcns called

$\%$ Hint: popupmenu controls usually have a white background on Windows.

$\% \quad$ See ISPC and COMPUTER.

if ispc \&\& isequal(get(hObject,'BackgroundColor'), get(0,'defaultUicontrolBackgroundColor')) set(hObject,'BackgroundColor','white');

end

end

$\%$--- Executes when figure 1 is resized.

function figure1_ResizeFcn(hObject, , handles)

$\%$ hObject handle to figure1 (see GCBO)

$\%$ reserved - to be defined in a future version of MATLAB

$\%$ handles structure with handles and user data (see GUIDATA)

end

function windspeed_text2_Callback(hObject, , handles)

$\%$ hObject handle to windspeed_text2 (see GCBO)

$\%$ reserved - to be defined in a future version of MATLAB

$\%$ handles structure with handles and user data (see GUIDATA)

$\%$ Hints: get(hObject,'String') returns contents of windspeed text2 as text

$\% \quad$ str2double(get(hObject,'String')) returns contents of windspeed_text2 as a double end

$\%$--- Executes during object creation, after setting all properties.

function windspeed_text2_CreateFcn(hObject, , handles)

$\%$ hObject handle to windspeed_text2 (see GCBO)

$\% \sim$ reserved - to be defined in a future version of MATLAB

$\%$ handles empty - handles not created until after all CreateFcns called

$\%$ Hint: edit controls usually have a white background on Windows.

$\% \quad$ See ISPC and COMPUTER.

if ispc \&\& isequal(get(hObject,'BackgroundColor'), get(0,'defaultUicontrolBackgroundColor')) set(hObject,'BackgroundColor','white');

end

end

function windspeed_text1_Callback(hObject, , handles)

$\%$ hObject handle to windspeed_text1 (see GCBO)

$\% \sim$ reserved - to be defined in a future version of MATLAB

$\%$ handles structure with handles and user data (see GUIDATA)

$\%$ Hints: get(hObject,'String') returns contents of windspeed_text1 as text

$\% \quad$ str2double(get(hObject,'String')) returns contents of windspeed_text1 as a double

end

$\%$--- Executes during object creation, after setting all properties. 
function windspeed_text1_CreateFcn(hObject, , handles)

$\%$ hObject handle to windspeed_text1 (see GCBO)

$\% \sim$ reserved - to be defined in a future version of MATLAB

$\%$ handles empty - handles not created until after all CreateFcns called

$\%$ Hint: edit controls usually have a white background on Windows.

$\% \quad$ See ISPC and COMPUTER.

if ispc \&\& isequal(get(hObject,'BackgroundColor'), get(0,'defaultUicontrolBackgroundColor')) set(hObject,'BackgroundColor','white');

end

end

$\%$--- Executes on selection change in windspeed_popup_dist.

function windspeed_popup_dist_Callback(hObject, $\sim$, handles)

$\%$ hObject handle to windspeed_popup_dist (see GCBO)

$\% \sim$ reserved - to be defined in a future version of MATLAB

$\%$ handles structure with handles and user data (see GUIDATA)

$\%$ Hints: contents = cellstr(get(hObject,'String')) returns windspeed_popup_dist contents as cell array

$\% \quad$ contents $\{$ get(hObject,'Value')\} returns selected item from windspeed_popup_dist

contents = cellstr(get(hObject,'String'));

windspeed_popchoice $=$ contents $\{$ get $($ hObject,'Value' $)\}$;

switch windspeed_popchoice

case 'Normal'

set(handles.windspeed_text1,'Enable','inactive') \%

set(handles.windspeed_text2,'Enable','inactive') \%

set(handles.windspeed_text1,'String','Mean');

set(handles.windspeed_text2,'String','Std Deviation');

set(handles.cq_pushbutton,'Enable','on')

set(handles.windspeed_text1,'TooltipString',")

set(handles.windspeed_text2,'TooltipString',")

case 'Beta'

set(handles.windspeed_text1,'Enable','inactive') \%

set(handles.windspeed_text2,'Enable','inactive') \%

set(handles.windspeed_text1,'String','a');

set(handles.windspeed_text2,'String','b');

set(handles.windspeed_text1,'TooltipString','shape parameter')

set(handles.windspeed_text2,'TooltipString','shape parameter')

set(handles.cq_pushbutton,'Enable','on')

case 'Uniform'

set(handles.windspeed_text1,'Enable','inactive') \%

set(handles.windspeed_text2,'Enable','inactive') \%

set(handles.windspeed_text1,'String','Upper Limit');

set(handles.windspeed_text2,'String','Lower Limit');

set(handles.cq_pushbutton,'Enable','on')

set(handles.windspeed_text1,'TooltipString',")

set(handles.windspeed_text2,'TooltipString',")

case 'Exponential'

set(handles.windspeed_text1,'Enable','inactive') \%

set(handles.windspeed_text2,'Enable','off') \%

set(handles.windspeed_text1,'String','Mean'); 


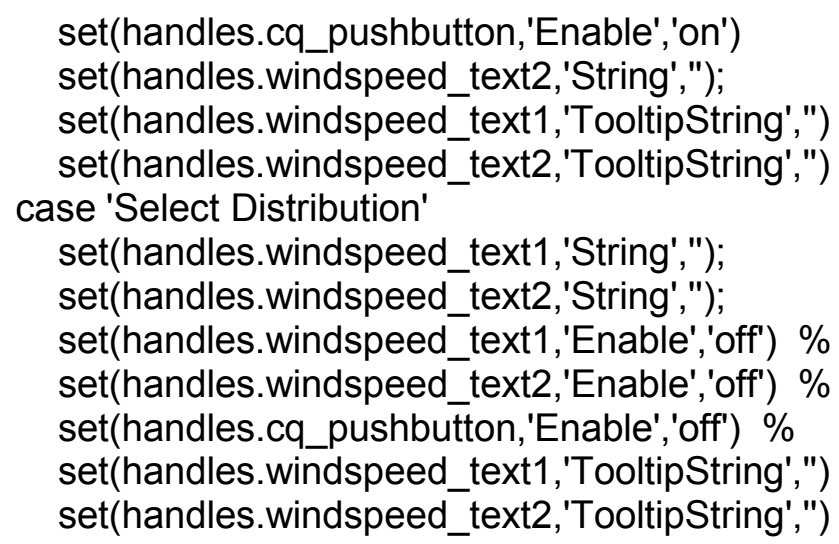

end

$\%$--- Executes during object creation, after setting all properties.

function windspeed_popup_dist_CreateFcn(hObject, , handles)

$\%$ hObject handle to windspeed_popup_dist (see GCBO)

$\% \sim$ reserved - to be defined in a future version of MATLAB

$\%$ handles empty - handles not created until after all CreateFcns called

$\%$ Hint: popupmenu controls usually have a white background on Windows.

$\% \quad$ See ISPC and COMPUTER.

if ispc \&\& isequal(get(hObject,'BackgroundColor'), get( 0 ,'defaultUicontrolBackgroundColor')) set(hObject,'BackgroundColor','white');

end

end

$\%$--- Executes when user attempts to close figure1.

function figure1_CloseRequestFcn(hObject, $\sim$, handles)

$\%$ hObject handle to figure1 (see GCBO)

$\% \sim$ reserved - to be defined in a future version of MATLAB

$\%$ handles structure with handles and user data (see GUIDATA)

$\%$ Hint: delete(hObject) closes the figure

exit_button = questdlg('Exit Now?','Exit SODA','Yes','No','Yes');

switch exit_button;

case 'Yes' delete(hObject);

case 'No'

return

end

end

$\%$

function random_gen_Callback(hObject, , handles)

$\%$ hObject handle to random_gen (see GCBO)

$\%$ reserved - to be defined in a future version of MATLAB

$\%$ handles structure with handles and user data (see GUIDATA)

rng('default'); 
msgbox('Random Number Generator has been reset','Reset');

end

$\%$--- If Enable == 'on', executes on mouse press in 5 pixel border.

$\%$--- Otherwise, executes on mouse press in 5 pixel border or over mar_text1.

function mar_text1_ButtonDownFcn(hObject, , handles)

$\%$ hObject handle to mar_text1 (see GCBO)

$\% \sim$ reserved - to be defined in a future version of MATLAB

$\%$ handles structure with handles and user data (see GUIDATA)

\% set(hObject,'String',",'Enable','on')

set(hObject,'Enable','on');

set(handles.mar_text1,'string',[]);

end

$\%$--- If Enable == 'on', executes on mouse press in 5 pixel border.

$\%$--- Otherwise, executes on mouse press in 5 pixel border or over mar_text2.

function mar_text2_ButtonDownFcn(hObject, , handles)

$\%$ hObject handle to mar_text2 (see GCBO)

$\% \sim$ reserved - to be defined in a future version of MATLAB

$\%$ handles structure with handles and user data (see GUIDATA)

set(hObject,'Enable','on');

set(handles.mar_text2,'string',[]);

end

$\%$--- If Enable == 'on', executes on mouse press in 5 pixel border.

$\%$--- Otherwise, executes on mouse press in 5 pixel border or over dr_text1.

function dr_text1_ButtonDownFcn(hObject, , handles)

$\%$ hObject handle to dr_text1 (see GCBO)

$\% \sim$ reserved - to be defined in a future version of MATLAB

$\%$ handles structure with handles and user data (see GUIDATA)

set(hObject,'Enable','on');

set(handles.dr_text1,'string',[]);

end

$\%$--- If Enable == 'on', executes on mouse press in 5 pixel border.

$\%$--- Otherwise, executes on mouse press in 5 pixel border or over dr_text2.

function dr_text2_ButtonDownFcn(hObject, , handles)

$\%$ hObject handle to dr_text2 (see GCBO)

$\% \sim$ reserved - to be defined in a future version of MATLAB

$\%$ handles structure with handles and user data (see GUIDATA)

set(hObject,'Enable','on');

set(handles.dr_text2,'string',[]);

end

$\%$--- If Enable == 'on', executes on mouse press in 5 pixel border.

$\%$--- Otherwise, executes on mouse press in 5 pixel border or over arf_text1.

function arf_text1_ButtonDownFcn(hObject, , handles)

$\%$ hObject handle to arf_text1 (see GCBO)

$\%$ reserved - to be defined in a future version of MATLAB

$\%$ handles structure with handles and user data (see GUIDATA)

set(hObject,'Enable','on'); 
set(handles.arf_text1,'string',[]);

end

$\%$--- If Enable == 'on', executes on mouse press in 5 pixel border.

$\%$--- Otherwise, executes on mouse press in 5 pixel border or over arf_text2.

function arf_text2_ButtonDownFcn(hObject, , handles)

$\%$ hObject handle to arf_text2 (see GCBO)

$\% \sim$ reserved - to be defined in a future version of MATLAB

$\%$ handles structure with handles and user data (see GUIDATA)

set(hObject,'Enable','on');

set(handles.arf_text2,'string',[]);

end

$\%$--- If Enable == 'on', executes on mouse press in 5 pixel border.

$\%$--- Otherwise, executes on mouse press in 5 pixel border or over rf_text1.

function rf_text1_ButtonDownFcn(hObject, , handles)

$\%$ hObject handle to if_text1 (see GCBO)

$\% \sim$ reserved - to be defined in a future version of MATLAB

$\%$ handles structure with handles and user data (see GUIDATA)

set(hObject,'Enable','on');

set(handles.rf_text1,'string',[]);

end

$\%$--- If Enable == 'on', executes on mouse press in 5 pixel border.

$\%$--- Otherwise, executes on mouse press in 5 pixel border or over rf_text2.

function rf_text2_ButtonDownFcn(hObject, , handles)

$\%$ hObject handle to if_text2 (see GCBO)

$\%$ reserved - to be defined in a future version of MATLAB

$\%$ handles structure with handles and user data (see GUIDATA)

set(hObject,'Enable','on');

set(handles.rf_text2,'string',[]);

end

$\%$--- If Enable == 'on', executes on mouse press in 5 pixel border.

$\%$--- Otherwise, executes on mouse press in 5 pixel border or over Ipf_text 1.

function Ipf_text1_ButtonDownFcn(hObject, , handles)

$\%$ hObject handle to Ipf_text1 (see GCBO)

$\% \sim$ reserved - to be defined in a future version of MATLAB

$\%$ handles structure with handles and user data (see GUIDATA)

set(hObject,'Enable','on');

set(handles.lpf_text1,'string',[]);

end

$\%$--- If Enable == 'on', executes on mouse press in 5 pixel border.

$\%$--- Otherwise, executes on mouse press in 5 pixel border or over lpf_text1.

function Ipf_text2_ButtonDownFcn(hObject, , handles)

$\%$ hObject handle to Ipf_text1 (see GCBO)

$\% \sim$ reserved - to be defined in a future version of MATLAB

$\%$ handles structure with handles and user data (see GUIDATA)

set(hObject,'Enable','on');

set(handles.lpf_text2,'string',[]);

end 
$\%$--- If Enable == 'on', executes on mouse press in 5 pixel border.

$\%$--- Otherwise, executes on mouse press in 5 pixel border or over height_text.

function height_text_ButtonDownFcn(hObject, , handles)

$\%$ hObject handle to height_text (see GCBO)

$\% \sim$ reserved - to be defined in a future version of MATLAB

$\%$ handles structure with handles and user data (see GUIDATA)

set(hObject,'Enable','on');

set(handles.height_text,'string',[]);

end

$\%$--- If Enable == 'on', executes on mouse press in 5 pixel border.

$\%$--- Otherwise, executes on mouse press in 5 pixel border or over windspeed_text1.

function windspeed_text1_ButtonDownFcn(hObject, , handles)

$\%$ hObject handle to windspeed_text1 (see GCBO)

$\% \sim$ reserved - to be defined in a future version of MATLAB

$\%$ handles structure with handles and user data (see GUIDATA)

set(hObject,'Enable','on');

set(handles.windspeed_text1,'string',[]);

end

$\%$--- If Enable == 'on', executes on mouse press in 5 pixel border.

$\%$--- Otherwise, executes on mouse press in 5 pixel border or over windspeed_text1.

function windspeed_text2_ButtonDownFcn(hObject, , handles)

$\%$ hObject handle to windspeed_text1 (see GCBO)

$\% \sim$ reserved - to be defined in a future version of MATLAB

$\%$ handles structure with handles and user data (see GUIDATA)

set(hObject,'Enable','on');

set(handles.windspeed_text2,'string',[]);

end

$\%$--- If Enable == 'on', executes on mouse press in 5 pixel border.

$\%$--- Otherwise, executes on mouse press in 5 pixel border or over dcf_text 1 .

function dcf_text1_ButtonDownFcn(hObject, , handles)

$\%$ hObject handle to dcf_text1 (see GCBO)

$\% \sim$ reserved - to be defined in a future version of MATLAB

$\%$ handles structure with handles and user data (see GUIDATA)

set(hObject,'Enable','on');

set(handles.dcf_text1,'string',[]);

end

$\%$--- If Enable == 'on', executes on mouse press in 5 pixel border.

$\%$--- Otherwise, executes on mouse press in 5 pixel border or over dcf_text1.

function dcf_text2_ButtonDownFcn(hObject, , handles)

$\%$ hObject handle to dcf_text1 (see GCBO)

$\% \sim$ reserved - to be defined in a future version of MATLAB

$\%$ handles structure with handles and user data (see GUIDATA)

set(hObject,'Enable','on');

set(handles.dcf_text2,'string',[]);

end

$\%$--- Executes during object deletion, before destroying properties.

function figure1_DeleteFcn(hObject, , handles)

$\%$ hObject handle to figure1 (see GCBO)

$\%$ reserved - to be defined in a future version of MATLAB 
$\%$ handles structure with handles and user data (see GUIDATA) end

$\%$--- Executes during object deletion, before destroying properties.

function mar_text1_DeleteFcn(hObject, , handles)

$\%$ hObject handle to mar_text1 (see GCBO)

$\%$ reserved - to be defined in a future version of MATLAB

$\%$ handles structure with handles and user data (see GUIDATA)

end

$\%$--- Executes on button press in fit_dist.

function fit_dist_Callback(hObject, , handles)

$\%$ hObject handle to fit_dist (see GCBO)

$\% \sim$ reserved - to be defined in a future version of MATLAB

$\%$ handles structure with handles and user data (see GUIDATA)

ced = getappdata $\left(0,{ }^{\prime}\right.$ ced');

col = get(handles.fit_dist,'backg');

set(handles.fit_dist,'str','RUNNING...','backg',[.2 .6 .6]);

pause(eps);

[ ,PD]=allfitdistBICPDF(ced,handles);

set(handles.fit_dist,'str','Fit Distribution','backg',col);

$\%$ assignin('base','PD', PD);

switch $\mathrm{PD}\{1,1\}$.DistributionName

case 'Generalized Extreme Value'

$\operatorname{msgbox}(\{\mathrm{PD}\{1,1\}$.DistributionName ['k =' num2str(PD\{1, 1\}.k)] ..

['Mean= ' num2str(PD $\{1,1\} . \mathrm{mu})]$ ['Sigma =' num2str(PD $\{1,1\}$.sigma)]\},'Best Fit','modal')

case 'Inverse Gaussian'

$\operatorname{msgbox}(\{\operatorname{PD}\{1,1\}$.DistributionName ['Mean =' num2str(PD $\{1,1\} . \mathrm{mu})] \ldots$

['Lambda= ' num2str(PD\{1, 1\}.lambda)]\},'Best Fit','modal')

case 'Lognormal'

$\operatorname{msgbox}(\{\mathrm{PD}\{1,1\}$.DistributionName ['Mean =' num2str(PD $\{1,1\} . \mathrm{mu})] \ldots$

['Sigma= ' num2str(PD $\{1,1\}$. sigma)] $\}$,'Best Fit','modal')

case 'Log-Logistic'

$\operatorname{msgbox}(\{P D\{1,1\}$.DistributionName ['Mean =' num2str(PD\{1, 1\}.mu)]...

['Sigma= ' num2str(PD $\{1,1\}$. sigma)] $\}$,'Best Fit','modal')

case 't Location-Scale'

$\operatorname{msgbox}(\{\mathrm{PD}\{1,1\}$.DistributionName ['Mean =' num2str(PD $\{1,1\} . \mathrm{mu})] \ldots$

['Sigma= ' num2str(PD $\{1,1\} . \operatorname{sigma})][$ 'Nu= ' num2str(PD $\{1,1\}$. nu $)]\}$,'Best Fit','modal')

case 'Gamma'

$\operatorname{msgbox}(\{\mathrm{PD}\{1,1\}$.DistributionName ['a =' num2str(PD\{1, 1\}.a)]...

$[$ 'b= ' num2str(PD\{1, 1\}.b)] \},'Best Fit','modal')

case 'Beta'

$\operatorname{msgbox}(\{P D\{1,1\}$.DistributionName ['a =' num2str(PD $\{1,1\} . a)] \ldots$

['b= ' num2str(PD\{1, 1\}.b)] \},'Best Fit','modal')

case 'Weibull'

$\operatorname{msgbox}(\{P D\{1,1\}$.DistributionName ['A =' num2str(PD $\{1,1\} . A)] \ldots$

['B= ' num2str(PD\{1, 1\}.B)] \},'Best Fit','modal')

case 'Generalized Pareto'

$\operatorname{msgbox}(\{\mathrm{PD}\{1,1\}$.DistributionName ['k =' num2str(PD $\{1,1\} . \mathrm{k})] \ldots$

['Sigma= ' num2str(PD $\{1,1\}$. sigma)] ['Theta= ' num2str(PD $\{1,1\}$.theta)]\},'Best Fit','modal') case 'Exponential' 


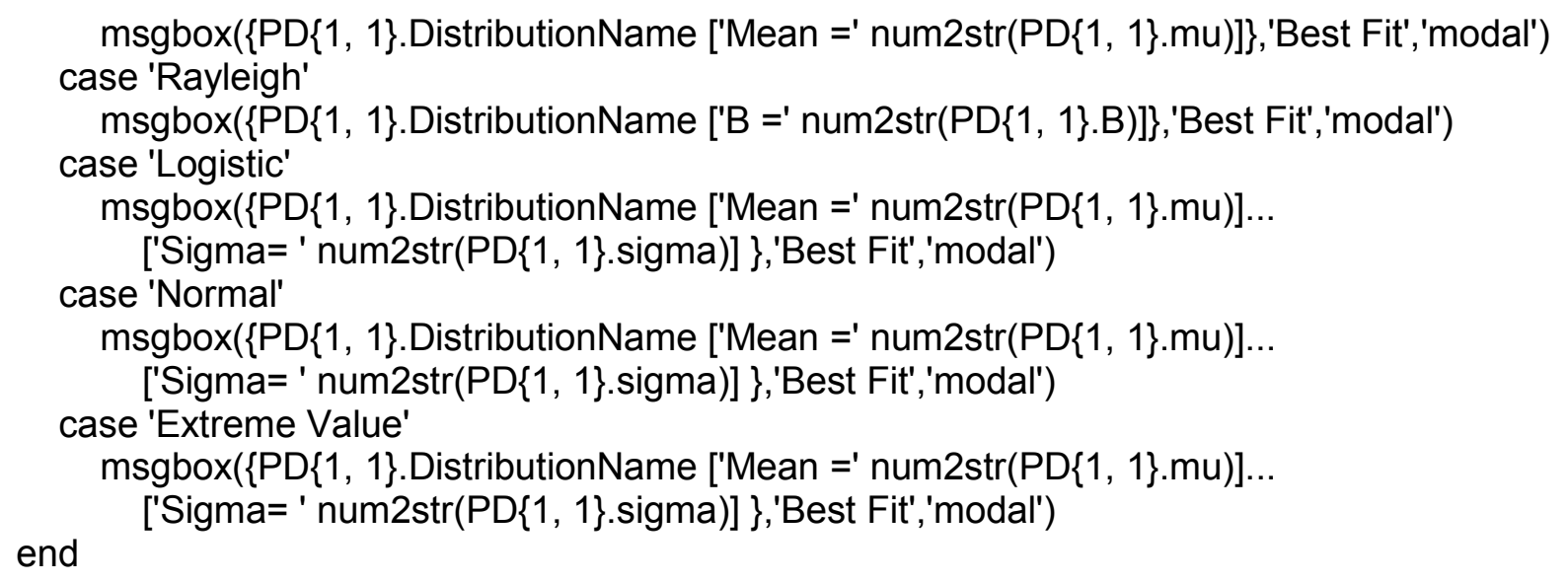

end

function $[D, P D]=$ allfitdistBICPDF(data, handles)

\%ALLFITDIST Fit all valid parametric probability distributions to data.

$\%$ [D PD] = ALLFITDIST (DATA) fits all valid parametric probability

$\%$ distributions to the data in vector DATA by BIC method, and returns

$\%$ a struct $\mathrm{D}$ of fitted distributions and parameters and a struct of

$\%$ objects PD representing the fitted distributions. PD is an object

$\%$ in a class derived from the ProbDist class.

$\%$

$\% \quad[\ldots]=$ ALLFITDIST (...,'PDF') or (...,'CDF') plots either the PDF or CDF

$\%$ of a subset of the fitted distribution. The distributions are plotted in

$\%$ order of fit, according to SORTBY.

$\%$

List of distributions it will try to fit

Beta

Exponential

Gamma

Inverse Gaussian

Logistic

Log-logistic

Lognormal

Normal

EXAMPLE 1

Given random data from an unknown continuous distribution, find the best distribution which fits that data, and plot the PDFs to compare graphically. data $=$ normrnd $(5,3,1 \mathrm{e} 4,1) ; \quad$ \%Assumed from unknown distribution [D PD] = allfitdist(data,'PDF'); \%Compute and plot results D(1) \%Show output from best fit 
warning('on','all'); \%Turn back on warnings

if numel(D)==0

errordlg('No distributions were found','Error'); return;

end

$\% \%$ Sort distributions

$\%$ prepares distribution fits according to BIC best fit to data indx1=1:length(D); \%ldentity Map

$[\sim$,indx1] =sort $([D . B I C])$;

$\mathrm{D}=\mathrm{D}($ ind $x 1) ; \mathrm{PD}=\mathrm{PDs}($ ind $x 1)$;

$\%$ Plot

plotfigs(data,D,PD, handles);

end

function plotfigs(data, D,PD, handles)

$\%$ Plot functionality for continuous case due to Jonathan Sullivan

$\%$ Modified by author for discrete case

\%Maximum number of distributions to include

\%max_num_dist=Inf; \%All valid distributions

max_num_dist=4;

cla(handles.axes1,'reset');

axes(handles.axes1);

$\% \%$ Probability Density / Mass Plot

\section{\%Continuous Data}

nbins $=\max (\min ($ length $($ data $) . / 10,100), 50) ;$

$x i=$ linspace $(\min ($ data $), \max ($ data $)$, nbins $)$;

$\mathrm{dx}=$ mean(diff(xi));

xi2 = linspace (min(data), $\max ($ data $)$, nbins $\left.^{*} 10\right)$;

$\mathrm{fi}=$ histc(data, $\mathrm{xi}-\mathrm{dx})$;

$\mathrm{fi}=\mathrm{fi} . / \mathrm{sum}(\mathrm{fi}) . / \mathrm{dx}$;

assignin('base','fitxi', xi);

assignin('base','fitfi2', fi);

inds = 1:min([max_num_dist,numel(PD)]);

ys = cellfun(@(PD) pdf(PD,xi2),PD(inds),'UniformOutput',0);

ys $=\operatorname{cat}(2, y s\{:\})$;

[r_gen,x_gen] = ksdensity (data);

plot(x_gen,r_gen,'LineWidth',3,'color','k');

$\%$ hold on; 
\% bar(xi,fi,'FaceColor','m','EdgeColor','m','BarWidth', 1);

hold on;

plot(xi2,ys,'LineWidth',1.5)

axis tight;

legend(['Random Generated',\{D(inds).DistName\}],'Location','NE');

xlabel('Commited Effective Dose (rem)');

ylabel('Probability Density');

title(['Probability Density Function with Imu =' num2str(mean(data)) ' Isigma =' num2str(std(data))]);

grid on;

end

$\%$--- Executes on key press with focus on mar_text1 and none of its controls.

function mar_text1_KeyPressFcn(hObject, , handles)

$\%$ hObject handle to mar_text1 (see GCBO)

$\% \sim$ structure with the following fields (see UICONTROL)

$\% \quad$ Key: name of the key that was pressed, in lower case

$\% \quad$ Character: character interpretation of the key(s) that was pressed

$\% \quad$ Modifier: name(s) of the modifier key(s) (i.e., control, shift) pressed

$\%$ handles structure with handles and user data (see GUIDATA)

end 
APPENDIX D:

\section{Damage Ratio Experiment Data}

\begin{tabular}{|c|c|c|}
\hline \multirow[b]{2}{*}{3 m drop (pint) } & \multicolumn{2}{|c|}{$\begin{array}{c}\text { Container } \\
\text { Break }\end{array}$} \\
\hline & Yes & No \\
\hline 1 & & 1 \\
\hline 2 & & 1 \\
\hline 3 & & 1 \\
\hline 4 & & 1 \\
\hline 5 & & 1 \\
\hline 6 & & 1 \\
\hline 7 & & 1 \\
\hline 8 & 1 & \\
\hline 9 & & 1 \\
\hline 10 & & 1 \\
\hline 11 & & 1 \\
\hline 12 & & 1 \\
\hline 13 & & 1 \\
\hline 14 & & 1 \\
\hline 15 & & 1 \\
\hline 16 & & 1 \\
\hline 17 & & 1 \\
\hline 18 & & 1 \\
\hline 19 & & 1 \\
\hline 20 & 1 & \\
\hline 21 & & 1 \\
\hline 22 & & 1 \\
\hline 23 & & 1 \\
\hline 24 & & 1 \\
\hline 25 & & 1 \\
\hline 26 & & 1 \\
\hline 27 & 1 & \\
\hline 28 & & 1 \\
\hline 29 & & 1 \\
\hline 30 & & 1 \\
\hline 31 & & 1 \\
\hline 32 & & 1 \\
\hline 33 & 1 & \\
\hline 34 & & 1 \\
\hline 35 & & 1 \\
\hline 36 & & 1 \\
\hline 37 & & 1 \\
\hline 38 & & 1 \\
\hline
\end{tabular}




\begin{tabular}{|c|c|c|}
\hline \multirow[b]{2}{*}{3 m drop (pint) } & \multicolumn{2}{|c|}{$\begin{array}{c}\text { Container } \\
\text { Break }\end{array}$} \\
\hline & Yes & No \\
\hline 39 & & 1 \\
\hline 40 & & 1 \\
\hline 41 & & 1 \\
\hline 42 & & 1 \\
\hline 43 & & 1 \\
\hline 44 & & 1 \\
\hline 45 & 1 & \\
\hline 46 & & 1 \\
\hline 47 & 1 & \\
\hline 48 & & 1 \\
\hline 49 & & 1 \\
\hline 50 & & 1 \\
\hline 51 & & 1 \\
\hline 52 & & 1 \\
\hline 53 & & 1 \\
\hline 54 & & 1 \\
\hline 55 & & 1 \\
\hline 56 & & 1 \\
\hline 57 & & 1 \\
\hline 58 & & 1 \\
\hline 59 & & 1 \\
\hline 60 & & 1 \\
\hline 61 & & 1 \\
\hline 62 & & 1 \\
\hline 63 & 1 & \\
\hline 64 & & 1 \\
\hline 65 & & 1 \\
\hline 66 & & 1 \\
\hline 67 & & 1 \\
\hline 68 & & 1 \\
\hline 69 & & 1 \\
\hline 70 & & 1 \\
\hline 71 & & 1 \\
\hline 72 & & 1 \\
\hline 73 & & 1 \\
\hline 74 & & 1 \\
\hline 75 & & 1 \\
\hline 76 & & 1 \\
\hline 77 & & 1 \\
\hline 78 & & 1 \\
\hline 79 & & 1 \\
\hline
\end{tabular}


INL/EXT-15-36669

Rev. 0 September 2015

\begin{tabular}{|c|c|c|}
\hline \multirow{2}{*}{ 3 m drop (pint) } & \multicolumn{2}{|c|}{$\begin{array}{c}\text { Container } \\
\text { Break }\end{array}$} \\
\cline { 2 - 3 } Yes & No \\
\hline 80 & & 1 \\
\hline 81 & & 1 \\
\hline 82 & & 1 \\
\hline 83 & & 1 \\
\hline 84 & & 1 \\
\hline 85 & & 1 \\
\hline 86 & & 1 \\
\hline 87 & 1 & \\
\hline 88 & & 1 \\
\hline 89 & 1 & \\
\hline 90 & & 1 \\
\hline 91 & & 1 \\
\hline 92 & & 1 \\
\hline 93 & & 1 \\
\hline 94 & & 1 \\
\hline 95 & & 1 \\
\hline 96 & & 1 \\
\hline 97 & & 1 \\
\hline 98 & & 1 \\
\hline 99 & & 1 \\
\hline 100 & & 1 \\
\hline & & \\
\hline Total & 9 & 91 \\
\hline & & \\
\hline
\end{tabular}

\begin{tabular}{|c|c|c|}
\hline \multirow{2}{*}{3 m drop (quart) } & \multicolumn{2}{|c|}{$\begin{array}{c}\text { Container } \\
\text { Break }\end{array}$} \\
\cline { 2 - 3 } & Yes & No \\
\hline 1 & & 1 \\
\hline 2 & & 1 \\
\hline 3 & & 1 \\
\hline 4 & & 1 \\
\hline 5 & & 1 \\
\hline 6 & 1 & \\
\hline 7 & & 1 \\
\hline 8 & & 1 \\
\hline 9 & & 1 \\
\hline 10 & & 1 \\
\hline 11 & & 1 \\
\hline 12 & & 1 \\
\hline 13 & & 1 \\
\hline 14 & 1 & \\
\hline 15 & & 1 \\
\hline
\end{tabular}


INL/EXT-15-36669

Rev. 0 September 2015

Page 125 of 129

\begin{tabular}{|c|c|c|}
\hline 16 & & 1 \\
\hline 17 & & 1 \\
\hline 18 & & 1 \\
\hline 19 & & 1 \\
\hline 20 & & 1 \\
\hline 21 & & 1 \\
\hline 22 & 1 & \\
\hline 23 & & 1 \\
\hline 24 & & 1 \\
\hline 25 & & 1 \\
\hline 26 & 1 & \\
\hline 27 & & 1 \\
\hline 28 & 1 & \\
\hline 29 & & 1 \\
\hline 30 & & 1 \\
\hline 31 & & 1 \\
\hline 32 & 1 & \\
\hline 33 & 1 & \\
\hline 34 & & 1 \\
\hline 35 & 1 & \\
\hline 36 & 1 & \\
\hline 37 & & 1 \\
\hline 38 & 1 & \\
\hline 39 & & 1 \\
\hline 40 & & 1 \\
\hline 41 & & 1 \\
\hline 42 & 1 & \\
\hline 43 & & 1 \\
\hline 44 & & 1 \\
\hline 45 & & 1 \\
\hline 46 & & 1 \\
\hline 47 & & 1 \\
\hline 48 & & 1 \\
\hline 49 & & 1 \\
\hline 50 & & 1 \\
\hline 51 & & 1 \\
\hline 52 & & 1 \\
\hline 53 & & 1 \\
\hline 54 & & 1 \\
\hline 55 & & 1 \\
\hline 56 & & 1 \\
\hline 57 & & 1 \\
\hline 58 & & 1 \\
\hline 59 & 1 & \\
\hline
\end{tabular}


INL/EXT-15-36669

Rev. 0 September 2015

Page 126 of 129

\begin{tabular}{|c|c|c|}
\hline 60 & & 1 \\
\hline 61 & 1 & \\
\hline 62 & & 1 \\
\hline 63 & & 1 \\
\hline 64 & & 1 \\
\hline 65 & 1 & \\
\hline 66 & & 1 \\
\hline 67 & & 1 \\
\hline 68 & & 1 \\
\hline 69 & & 1 \\
\hline 70 & & 1 \\
\hline 71 & & 1 \\
\hline 72 & & 1 \\
\hline 73 & & 1 \\
\hline 74 & & 1 \\
\hline 75 & 1 & \\
\hline 76 & 1 & \\
\hline 77 & & 1 \\
\hline 78 & 1 & \\
\hline 79 & & 1 \\
\hline 80 & & 1 \\
\hline 81 & 1 & \\
\hline 82 & 1 & \\
\hline 83 & & 1 \\
\hline 84 & 1 & \\
\hline 85 & & 1 \\
\hline 86 & & 1 \\
\hline 87 & & 1 \\
\hline 88 & 1 & \\
\hline 89 & 1 & \\
\hline 90 & 1 & \\
\hline 91 & & 1 \\
\hline 92 & 1 & \\
\hline 93 & & 1 \\
\hline 94 & & 1 \\
\hline 95 & 1 & \\
\hline 96 & & 1 \\
\hline 97 & 1 & \\
\hline 98 & 1 & \\
\hline 99 & & 1 \\
\hline 100 & & 1 \\
\hline Total & 27 & 73 \\
\hline
\end{tabular}




\begin{tabular}{|c|c|c|}
\hline \multirow[b]{2}{*}{$1 \mathrm{~m}$ drop (pint) } & \multicolumn{2}{|c|}{$\begin{array}{c}\text { Container } \\
\text { Break }\end{array}$} \\
\hline & Yes & No \\
\hline 1 & & 1 \\
\hline 2 & & 1 \\
\hline 3 & & 1 \\
\hline 4 & & 1 \\
\hline 5 & & 1 \\
\hline 6 & & 1 \\
\hline 7 & & 1 \\
\hline 8 & & 1 \\
\hline 9 & & 1 \\
\hline 10 & & 1 \\
\hline 11 & & 1 \\
\hline 12 & & 1 \\
\hline 13 & & 1 \\
\hline 14 & & 1 \\
\hline 15 & & 1 \\
\hline 16 & & 1 \\
\hline 17 & & 1 \\
\hline 18 & & 1 \\
\hline 19 & & 1 \\
\hline 20 & & 1 \\
\hline 21 & & 1 \\
\hline 22 & & 1 \\
\hline 23 & & 1 \\
\hline 24 & & 1 \\
\hline 25 & & 1 \\
\hline 26 & & 1 \\
\hline 27 & & 1 \\
\hline 28 & & 1 \\
\hline 29 & & 1 \\
\hline 30 & & 1 \\
\hline 31 & & 1 \\
\hline 32 & & 1 \\
\hline 33 & & 1 \\
\hline 34 & & 1 \\
\hline 35 & & 1 \\
\hline 36 & & 1 \\
\hline 37 & & 1 \\
\hline 38 & & 1 \\
\hline 39 & & 1 \\
\hline 40 & & 1 \\
\hline 41 & 1 & \\
\hline
\end{tabular}




\begin{tabular}{|c|c|c|}
\hline \multirow[b]{2}{*}{$1 \mathrm{~m}$ drop (pint) } & \multicolumn{2}{|c|}{$\begin{array}{c}\text { Container } \\
\text { Break }\end{array}$} \\
\hline & Yes & No \\
\hline 42 & 1 & \\
\hline 43 & 1 & \\
\hline 44 & & 1 \\
\hline 45 & 1 & \\
\hline 46 & & 1 \\
\hline 47 & 1 & \\
\hline 48 & 1 & \\
\hline 49 & 1 & \\
\hline 50 & & 1 \\
\hline 51 & & 1 \\
\hline 52 & & 1 \\
\hline 53 & 1 & \\
\hline 54 & & 1 \\
\hline 55 & & 1 \\
\hline 56 & & 1 \\
\hline 57 & & 1 \\
\hline 58 & & 1 \\
\hline 59 & & 1 \\
\hline 60 & 1 & \\
\hline 61 & & 1 \\
\hline 62 & & 1 \\
\hline 63 & & 1 \\
\hline 64 & 1 & \\
\hline 65 & & 1 \\
\hline 66 & & 1 \\
\hline 67 & & 1 \\
\hline 68 & 1 & \\
\hline 69 & & 1 \\
\hline 70 & 1 & \\
\hline 71 & & 1 \\
\hline 72 & & 1 \\
\hline 73 & & 1 \\
\hline 74 & 1 & \\
\hline 75 & 1 & \\
\hline 76 & 1 & \\
\hline 77 & & 1 \\
\hline 78 & 1 & \\
\hline 79 & & 1 \\
\hline 80 & 1 & \\
\hline 81 & 1 & \\
\hline 82 & & 1 \\
\hline
\end{tabular}


INL/EXT-15-36669

Rev. 0 September 2015

Page 129 of 129

\begin{tabular}{|c|c|c|}
\hline \multirow{2}{*}{ 1 m drop (pint) } & \multicolumn{2}{|c|}{$\begin{array}{c}\text { Container } \\
\text { Break }\end{array}$} \\
\cline { 2 - 3 } Yes & No \\
\hline 83 & 1 & \\
\hline 84 & & 1 \\
\hline 85 & & 1 \\
\hline 86 & 1 & \\
\hline 87 & & 1 \\
\hline 88 & 1 & \\
\hline 89 & & 1 \\
\hline 90 & & 1 \\
\hline 91 & 1 & \\
\hline 92 & 1 & \\
\hline 93 & & 1 \\
\hline 94 & 1 & \\
\hline 95 & & 1 \\
\hline 96 & & 1 \\
\hline 97 & & 1 \\
\hline 98 & & 1 \\
\hline 99 & 1 & \\
\hline 100 & 1 & \\
\hline Total & 26 & 74 \\
\hline & & \\
\hline
\end{tabular}

Appendix D 